\title{
Total Synthesis and Structure Revision of (-)-Illisimonin A, a Neuroprotective Sesquiterpenoid from the Fruits of Illicium simonsii
}

Alexander S. Burns and Scott D. Rychnovsky*

Department of Chemistry, 1102 Natural Sciences II, University of California, Irvine, Irvine, California 92697

\section{Supporting Information}

Table of Contents

General experimental

Experimentals and compound characterization (racemic)

Experimentals and compound characterization (enantioenriched)

Illisimonin A tabulated NMR data

(-)-Illisimonin A CD comparison

NMR assignments of compounds 13, 17, 21, and 23

Experimental references

Calculations for compounds 8, 9, 10 and the endo TS

Calculated ECD for $1 R$ illisimonin A

Computational references

NMR spectra

HPLC traces
Page

S2

S3

S25

S32

S33

S34

S41

S42

S48

S50

S51

S116 


\section{General Experimental Details}

Chemical shifts $(\delta)$ were referenced to either TMS or the residual solvent peak. The ${ }^{1} \mathrm{H}$ NMR spectra data are presented as follows: chemical shift, multiplicity $(\mathrm{s}=$ singlet, $\mathrm{d}=$ doublet, $\mathrm{t}=$ triplet, $\mathrm{q}=$ quartet, $\mathrm{m}=$ multiplet, $\mathrm{dd}=$ doublet of doublets, $\mathrm{ddd}=$ doublet of doublet of doublets, $\mathrm{dddd}=$ doublet of doublet of doublet of doublets, $\mathrm{dt}=$ doublet of triplets, $\mathrm{dq}=$ doublet of quartets, $\mathrm{ddq}=$ doublet of doublet of quartets, app. = apparent), coupling constant(s) in hertz (Hz), and integration. High-resolution mass spectrometry was performed using ESI-TOF.

Unless otherwise stated, synthetic reactions were carried out in flame- or oven-dried glassware under an atmosphere of argon. All commercially available reagents were used as received unless stated otherwise. Solvents were purchased as ACS grade or better and as HPLC-grade and passed through a solvent purification system equipped with activated alumina columns prior to use. Thin layer chromatography (TLC) was carried out using glass plates coated with a $250 \mu \mathrm{m}$ layer of 60 Å silica gel. TLC plates were visualized with a UV lamp at $254 \mathrm{~nm}$, or by staining with potassium permanganate or Hanessian's stain. ${ }^{1}$ Liquid chromatography was performed using forced flow (flash chromatography) with an automated purification system on prepacked silica gel $\left(\mathrm{SiO}_{2}\right)$ columns unless otherwise stated. Electrospray ionization mass spectrometry (ESI-MS) was analyzed in positive mode with flow injection. Circular dichroism was carried out on a Jasco J810 spectropolarimeter with a $0.1 \mathrm{~cm}$ cell at room temperature with the following parameters: speed $50 \mathrm{~nm} / \mathrm{min}$; D.I.T. $2 \mathrm{sec}$; bandwidth $5 \mathrm{~nm}$.

\section{Chemicals}

All purchased chemicals were used without further purification unless otherwise noted. $\mathrm{CDCl}_{3}$ 
was purchased from Cambridge Isotope Laboratories. $\mathrm{Fe}(R, R) \mathrm{PDP}$ and $\mathrm{Fe}(S, S) \mathrm{PDP}$ were prepared as described, and stored in a nitrogen filled glove box prior to use. ${ }^{2}$

\section{Compound Synthesis and Characterization}

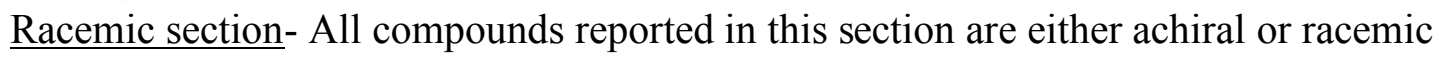

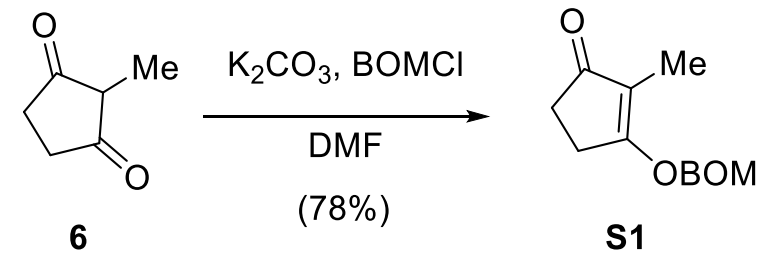

3-((Benzyloxy)methoxy)-2-methylcyclopent-2-en-1-one (S1). 2-Methylcyclopenta-1,3dione (6) (12.60 g, $69.7 \mathrm{mmol}), \mathrm{K}_{2} \mathrm{CO}_{3}(29 \mathrm{~g}, 0.20 \mathrm{~mol})$, and $\mathrm{BOMCl}$ (approximately 78\% by NMR analysis) $(15.0 \mathrm{~mL}$, ca. $83.7 \mathrm{mmol})$ were combined in a round-bottom flask. DMF $(157 \mathrm{~mL})$ was added and the reaction mixture was stirred at ambient temperature for $24 \mathrm{~h}$. EtOAc (150 mL) was added, and the reaction mixture was transferred to a separatory funnel and washed with $\mathrm{H}_{2} \mathrm{O}$ $(2 \times 150 \mathrm{~mL})$. The organic layer was dried over $\mathrm{MgSO}_{4}$ and concentrated in vacuo. The residue was diluted with $\mathrm{CH}_{2} \mathrm{Cl}_{2}(150 \mathrm{~mL})$ and washed with $\mathrm{H}_{2} \mathrm{O}(4 \times 150 \mathrm{~mL})$ to remove most of the remaining DMF. The organic layer was dried over $\mathrm{MgSO}_{4}$ and concentrated in vacuo. The crude mixture was purified by flash column chromatography (55\% EtOAc in hexanes) on silica to afford S1 $(12.60 \mathrm{~g}, 78 \%)$ as an orange oil which would reversibly solidify in a $-20{ }^{\circ} \mathrm{C}$ freezer.

$\mathbf{R}_{f}=0.27$ (60\% EtOAc in hexanes), visualized by UV

${ }^{1}$ H NMR $\left(500 \mathrm{MHz}, \mathrm{CDCl}_{3}\right) \delta$ 7.39-7.26 (m, 5H), $5.30(\mathrm{~s}, 2 \mathrm{H}), 4.70(\mathrm{~s}, 2 \mathrm{H}), 2.77-2.65(\mathrm{~m}, 2 \mathrm{H})$, 2.46-2.36 (m, 2H), $1.63(\mathrm{~s}, 3 \mathrm{H})$.

${ }^{13} \mathbf{C}\left\{{ }^{1} \mathbf{H}\right\}$ NMR $\left(126 \mathrm{MHz}, \mathrm{CDCl}_{3}\right) \delta$ 205.7, 182.1, 136.6, 128.6, 128.2, 128.0, 118.1, 91.7, 71.1, $33.6,24.8,6.0$.

HRMS (ESI/TOF) m/z: [M+H] $]^{+}$Calcd for $\mathrm{C}_{14} \mathrm{H}_{17} \mathrm{O}_{3}$ 233.1173; Found 233.1176. 


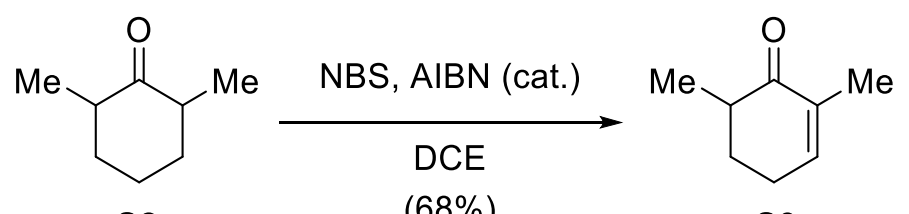

S2

$(68 \%)$

S3

2,6-Dimethylcyclohex-2-en-1-one (S3). This compound was prepared following a literature procedure modified for safety and reproducibility. ${ }^{3}$ A 3-neck, 1-L round bottom flask was equipped with two reflux condensers and a large magnetic stir bar to allow efficient stirring. An argon line was placed in one of the condensers, and an outlet attached to a bubbler was placed in the other. Argon was passed through the reaction flask throughout the course of the reaction. The flask was charged with 2,6-dimethylcyclohexanone (cis:trans mixture) $(30.0 \mathrm{~mL}, 0.219 \mathrm{~mol}), \mathrm{N}-$ bromosuccinimide (43.1 g, $0.242 \mathrm{~mol})$, AIBN (150 mg, $0.913 \mathrm{mmol})$, and DCE (180 mL). The flask was heated with a heat gun until the reaction initiated (vigorous exotherm and generation of gas), at which time the heat was removed. After ca. 5-10 min, the bromination was complete, and the reaction mixture had cooled down below the boiling point of DCE. The solution was then heated to reflux in an oil bath overnight (ca. $15 \mathrm{~h}$ ). The following morning, the mixture was allowed to cool to RT, transferred to a separatory funnel, and washed successively with saturated $\mathrm{Na}_{2} \mathrm{~S}_{2} \mathrm{O}_{3}$ (aq) (200 mL), 2M NaOH (aq) $(3 \times 200 \mathrm{~mL})$ and then brine $(200 \mathrm{~mL})$. Each aqueous phase was extracted one additional time with $\mathrm{CHCl}_{3}(50 \mathrm{~mL})$. The combined organic layers were dried over $\mathrm{Na}_{2} \mathrm{SO}_{4}$ and concentrated in vacuo. The resulting oil was then purified by short path distillation to yield $19.57 \mathrm{~g}$ of a clear oil containing product $\mathbf{S 3}$ along with $5 \mathrm{~mol} \%$ of 2,6dimethylcyclohexanone (S2) and 1.5 mol\% DCE. (corrected yield: $18.42 \mathrm{~g}, 68 \%$ ). Spectral data was consistent with literature report. The mixture was taken on to the next step without additional purification.

$\mathbf{R}_{f}=0.29$ (5\% EtOAc in hexanes) visualized by UV 
${ }^{1}$ H NMR $\left(500 \mathrm{MHz}, \mathrm{CDCl}_{3}\right) \delta$ 6.68-6.60 (m, 1H), 2.37-2.27 (m, 3H), 1.99 (dddd, $J=9.0,5.8$, 4.8, 1.1 Hz, 1H), 1.73-1.70 (m, 3H), 1.70-1.63 (m, 1H), 1.09 (d, J=6.9 Hz, 3H).

${ }^{13} \mathbf{C}\left\{{ }^{1} \mathbf{H}\right\}$ NMR $\left(126 \mathrm{MHz}, \mathrm{CDCl}_{3}\right) \delta 202.6,144.6,135.1,41.7,31.3,25.4,16.2,15.3$.

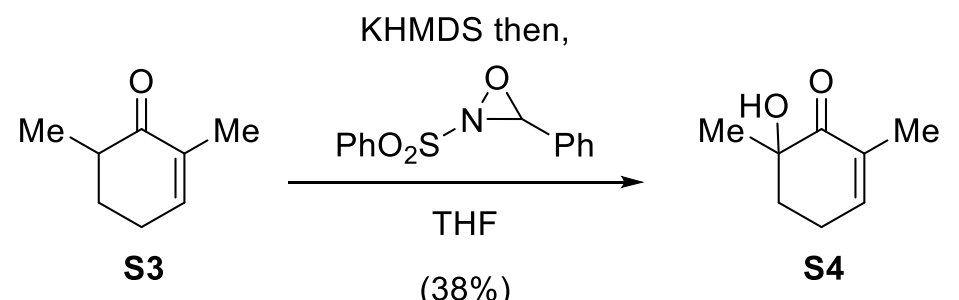

6-Hydroxy-2,6-dimethylcyclohex-2-en-1-one (S4). This compound was prepared using a modified literature procedure. ${ }^{3 \mathrm{~b}}$ A 1-L round bottom flask was charged with solid KHMDS (34.33 g, $172.1 \mathrm{mmol})$. THF $(500 \mathrm{~mL})$ was added and the flask was cooled to $-78^{\circ} \mathrm{C}$. A solution of $2,6-$ dimethylcyclohex-2-en-1-one (S3) (20.33 g, $163.7 \mathrm{mmol})$ in THF $(200 \mathrm{~mL})$ was cooled to $-78{ }^{\circ} \mathrm{C}$, and then added to the base via canula over a period of $10 \mathrm{~min}$. The reaction mixture was then stirred for an additional $45 \mathrm{~min}$.

In a separate 2-L round bottom flask, $( \pm$ )-phenyl-2-(phenylsulfonyl)-1,2-oxaziridine (44.9 g, $171.9 \mathrm{mmol})$ in THF $(300 \mathrm{~mL})$ was cooled to $-78^{\circ} \mathrm{C}$. The potassium enolate was added via canula over a period of $10 \mathrm{~min}$, and the solution was then stirred for an additional $45 \mathrm{~min}$. Saturated $\mathrm{NH}_{4} \mathrm{Cl}(800 \mathrm{~mL})$ was added, and the reaction mixture was allowed to warm to $\mathrm{RT}$, and transferred to a separatory funnel. The organic layer was collected, and the aqueous layer was washed with $\mathrm{Et}_{2} \mathrm{O}(2 \times 300 \mathrm{~mL})$. The combined organic layers were dried over $\mathrm{MgSO}_{4}$, and concentrated in vacuo. 1:1 $\mathrm{Et}_{2} \mathrm{O}$ :pentanes $(125 \mathrm{~mL})$ was added to the crude reaction mixture, and the slurry was then placed in $\mathrm{a}-25^{\circ} \mathrm{C}$ freezer for at least one hour. The resulting solids were filtered off, and washed with cold 1:1 Et ${ }_{2} \mathrm{O}$ :pentanes $(3 \times 75 \mathrm{~mL})$. The filtrate was concentrated in vacuo, and the orange liquid was then purified by short-path distillation, yielding a mixture of product and 
benzaldehyde. The mixture was then purified by flash column chromatography on silica (25\% EtOAc in hexanes, until the benzaldehyde eluted, then 60\% EtOAc in hexanes), to afford S4 (8.73 g, 38\%) as a pale yellow oil. Spectral data was consistent with literature.

$\mathbf{R}_{\boldsymbol{f}}=0.30$ (20\% EtOAc in hexanes), visualized by UV

${ }^{1} \mathbf{H}$ NMR $\left(500 \mathrm{MHz}, \mathrm{CDCl}_{3}\right) \delta$ 6.69-6.63 (m, 1H), $3.73(\mathrm{~s}, 1 \mathrm{H}), 2.42-2.36(\mathrm{~m}, 2 \mathrm{H}), 2.11-1.98(\mathrm{~m}$, $2 \mathrm{H}), 1.80(\mathrm{~s}, 3 \mathrm{H}), 1.28(\mathrm{~s}, 3 \mathrm{H})$.

${ }^{13} \mathbf{C}\left\{{ }^{1} \mathbf{H}\right\} \mathbf{N M R}\left(126 \mathrm{MHz}, \mathrm{CDCl}_{3}\right) \delta 203.4,145.7,132.8,73.2,35.9,24.5,24.3,15.9$.

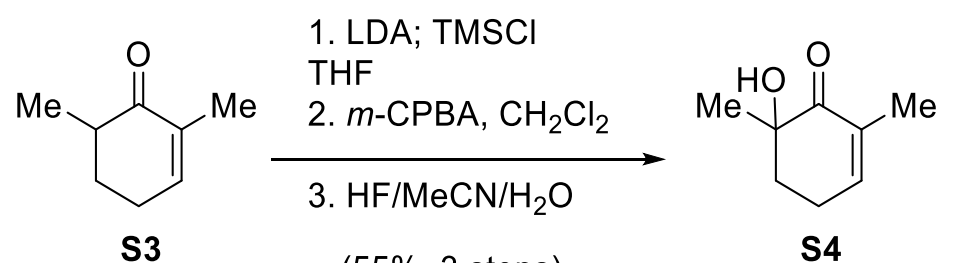

s3

(55\%, 3 steps)

S4

An alternative 3-step procedure was developed to access S4: Diisopropylamine $(26.7 \mathrm{~mL}$, $156 \mathrm{mmol})$ in THF $(250 \mathrm{~mL})$ was cooled to $-78^{\circ} \mathrm{C} . n$-BuLi $(2.30 \mathrm{M}$ in hexanes $)(75.2 \mathrm{~mL}, 173$ mmol) was added over $5 \mathrm{~min}$. The solution was warmed to $0{ }^{\circ} \mathrm{C}$ for $5 \mathrm{~min}$ then back to $-78{ }^{\circ} \mathrm{C}$. Enone S3 (18.42 g, $148.3 \mathrm{mmol}$ ) containing an additional $5 \mathrm{~mol} \%$ of 2,6-dimethylcyclohexanone (S2) and $1.5 \mathrm{~mol} \%$ DCE was added dropwise over a period of $5 \mathrm{~min}$. The solution was stirred for an additional $15 \mathrm{~min}$. TMSCl $(24.0 \mathrm{~mL}, 189 \mathrm{mmol})$ was added, and the reaction mixture was allowed to warm to RT over $45 \mathrm{~min}$. The solvent was removed via a rotary evaporator. Pentanes $(300 \mathrm{~mL})$ were added, and the solids were filtered off and washed with additional pentanes (100 $\mathrm{mL})$. The filtrate was transferred to a separatory funnel, and washed with acetonitrile $(500 \mathrm{~mL})$. The acetonitrile layer was washed with pentanes $(5 \times 100 \mathrm{~mL})$. The combined pentane washes were concentrated in vacuo, and the crude silyl enol ether $\mathbf{S 5}$ was used without further purification.

Silyl enol ether $\mathbf{S 5}$ was dissolved in $\mathrm{CH}_{2} \mathrm{Cl}_{2}(800 \mathrm{~mL}$; used directly from the bottle without 
attempt to further dry the solvent). This solution was placed in a 3-L RB flask equipped with overhead stirrer, and was open to the atmosphere. ${ }^{4}$ Solid $\mathrm{NaHCO}_{3}(38 \mathrm{~g}, 0.45 \mathrm{~mol})$ was added. m-CPBA (ca. 70\% with $\left.\mathrm{H}_{2} \mathrm{O}\right)(38.5 \mathrm{~g}$, ca. $156 \mathrm{mmol})$ was added portionwise to the stirred solution of silyl enol ether over a period of $30 \mathrm{~min}$. After the starting material had been consumed (as verified by TLC analysis), saturated $\mathrm{Na}_{2} \mathrm{~S}_{2} \mathrm{O}_{3}(800 \mathrm{~mL})$ was added and the solution was stirred for 30 min until all solids dissolved. The mixture was transferred to a separatory funnel, and the organic layer was collected. The aqueous layer was washed with $\mathrm{CHCl}_{3}(2 \times 200 \mathrm{~mL})$, and the combined organic extracts were dried over $\mathrm{Na}_{2} \mathrm{SO}_{4}$ and concentrated in vacuo. The crude silyl alcohol S6 was used without further purification.

HF:MeCN:H $\mathrm{H}_{2} \mathrm{O}$ (1:8:1 by volume) $(150 \mathrm{~mL})$ was added to crude silyl alcohol $\mathbf{S 6}$ under an atmosphere of air. After $20 \mathrm{~min}$, all of the starting material had been consumed as judged by TLC analysis. Saturated $\mathrm{NaHCO}_{3}(\mathrm{aq})(100 \mathrm{~mL})$ was added slowly to the reaction mixture. Solid $\mathrm{NaHCO}_{3}$ was added in approximately 1 gram portions to the vigorously stirred reaction mixture until bubbling had ceased. The mixture was then stirred for an additional $5 \mathrm{~min}$, then transferred to a separatory funnel. $\mathrm{H}_{2} \mathrm{O}(200 \mathrm{~mL})$ was added followed by $\mathrm{Et}_{2} \mathrm{O}(300 \mathrm{~mL})$. The organic layer was collected, and the aqueous layer was washed with additional $\mathrm{Et}_{2} \mathrm{O}(2 \times 100 \mathrm{~mL})$. The combined organic extracts were washed with brine $(200 \mathrm{~mL})$, dried over $\mathrm{MgSO}_{4}$, concentrated in vacuo, and purified via flash column chromatography on silica $(10-30 \%$ EtOAc in hexanes gradient) to afford S4 (11.46 $\mathrm{g}, 55 \%)$ as a yellow oil. The product was modestly volatile.

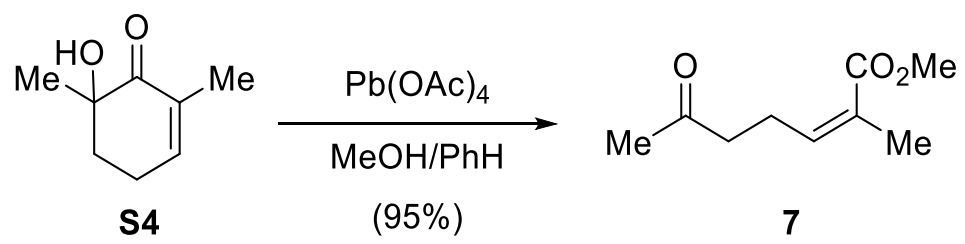

Methyl (Z)-2-methyl-6-oxohept-2-enoate (7). Ketone 7 was synthesized using a modified 
literature procedure. ${ }^{3 \mathrm{~b}} \mathrm{~Pb}(\mathrm{OAc})_{4}(42.2 \mathrm{~g}, 95.3 \mathrm{mmol})$ was dissolved in benzene $(540 \mathrm{~mL})$ and MeOH (200 mL). Alcohol S4 (11.11 g, $79.4 \mathrm{mmol})$ as a solution in benzene (100 mL) was added to the solution, and the mixture was heated to $40{ }^{\circ} \mathrm{C}$ for $3 \mathrm{~h}$. After the starting material was consumed as verified by TLC, the stir bar was removed, and most of the solvent was removed under reduced pressure. Once solid began to crash out, silica gel (75 g) was added, and the remaining solvent was removed on the rotary evaporator. Complete removal of the solvent was necessary, and could be achieved either through prolonged evaporation times (30 min), or an additional azeotrope of the solvent/silica mixture with $\mathrm{CH}_{2} \mathrm{Cl}_{2}(150 \mathrm{~mL})$.

A slurry of silica gel (200 g) and 40\% EtOAc in hexanes mixture was added to a column. The product and lead impregnated silica was loaded on top of the plug, and 40\% EtOAc in hexanes mixture $(1.5 \mathrm{~L})$ was passed through the plug. The solvent was transferred to a separatory funnel, and washed with saturated $\mathrm{NaHCO}_{3}(\mathrm{aq})(500 \mathrm{~mL})$. The organic layer was dried over $\mathrm{Na}_{2} \mathrm{SO}_{4}$, and concentrated in vacuo to yield 7 as a pale yellow oil (12.76 g, 95\%). Spectral data was consistent with the literature.

$\mathbf{R}_{\boldsymbol{f}}=0.46$ (25\% EtOAc in hexanes), visualized by $\mathrm{UV}$ or $\mathrm{KMnO}_{4}$

${ }^{1} \mathbf{H}$ NMR $\left(500 \mathrm{MHz}, \mathrm{CDCl}_{3}\right) \delta 5.93$ (tq, $\left.J=7.6,1.5 \mathrm{~Hz}, 1 \mathrm{H}\right), 3.73$ (s, 3H), 2.68 (app. q, $J=7.2$ $\mathrm{Hz}, 2 \mathrm{H}), 2.55(\mathrm{t}, J=7.2 \mathrm{~Hz}, 2 \mathrm{H}), 2.14(\mathrm{~s}, J=7.6 \mathrm{~Hz}, 3 \mathrm{H}), 1.87$ (q, $J=1.3 \mathrm{~Hz}, 3 \mathrm{H})$.

${ }^{13} \mathbf{C}\left\{{ }^{1} \mathbf{H}\right\} \mathbf{N M R}\left(126 \mathrm{MHz}, \mathrm{CDCl}_{3}\right) \delta 208.1,168.3,141.3,128.1,51.4,43.2,29.9,24.0,20.7$.

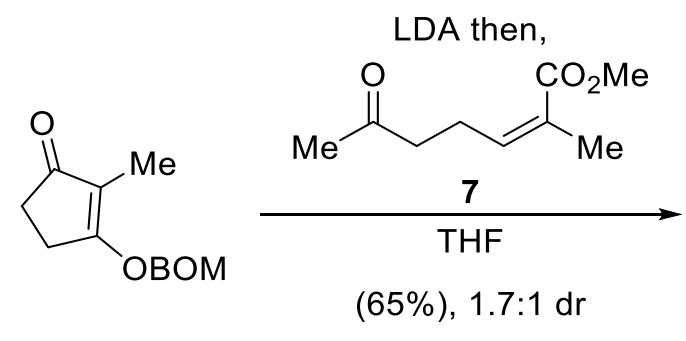

s1

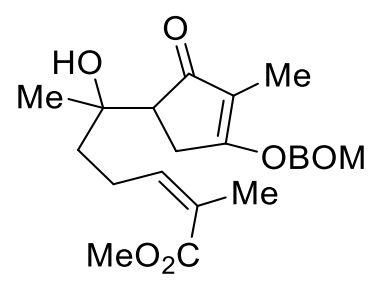

11 
Aldol adduct 11. Diisopropylamine $(5.12 \mathrm{~mL}, 36.3 \mathrm{mmol})$ was dissolved in THF $(56 \mathrm{~mL})$ and cooled to $-78{ }^{\circ} \mathrm{C}$. $n$-BuLi (2.17 $\mathrm{M}$ in hexanes) $(14.0 \mathrm{~mL}, 30.4 \mathrm{mmol})$ was added. The solution was warmed to $0^{\circ} \mathrm{C}$ for $5 \mathrm{~min}$, then cooled back to $-78{ }^{\circ} \mathrm{C}$. Cyclopentenone $\mathbf{S 1}(7.04 \mathrm{~g}, 30.3 \mathrm{mmol})$ as a solution in THF $(56 \mathrm{~mL})$ was added over a period of $5 \mathrm{~min}$. After $1 \mathrm{~h}$, the reaction mixture was warmed to $-40{ }^{\circ} \mathrm{C}$ for $15 \mathrm{~min}$ then cooled back to $-78^{\circ} \mathrm{C}$. Ketone $7(5.41 \mathrm{~g}, 31.8 \mathrm{mmol})$ as a solution in THF $(10 \mathrm{~mL})$ was added rapidly. After $30 \mathrm{~min}$, saturated $\mathrm{NH}_{4} \mathrm{Cl}(\mathrm{aq})(100 \mathrm{~mL})$ was added, and the mixture was allowed to warm to RT. The mixture was transferred to a separatory funnel, and the organic layer was collected. The aqueous layer was washed with $\mathrm{Et}_{2} \mathrm{O}(2 \times 50$ $\mathrm{mL})$. The combined organic layers were washed with brine $(100 \mathrm{~mL})$, dried over $\mathrm{MgSO}_{4}$, and passed through a small plug of silica. The silica was washed with EtOAc $(150 \mathrm{~mL})$. The filtrate was concentrated in vacuo and purified by flash column chromatography on silica (25-70\% EtOAc in hexanes gradient), to afford $\mathbf{1 1}(8.06 \mathrm{~g}, 65 \%)$ as a 1.7:1 mixture of diastereomers. The mixture contained some minor impurities which were taken forward to the next step. Each diastereomer could be isolated by further silica chromatography.

Major diastereomer:

$\mathbf{R}_{\boldsymbol{f}}=0.36(33 \%$ EtOAc in hexanes), visualized by UV

${ }^{1} \mathbf{H}$ NMR $\left(500 \mathrm{MHz}, \mathrm{CDCl}_{3}\right) \delta{ }^{1} \mathrm{H} \mathrm{NMR}\left(500 \mathrm{MHz}, \mathrm{CDCl}_{3}\right) \delta 7.38-7.27(\mathrm{~m}, 5 \mathrm{H}), 5.98(\mathrm{t}, J=7.6$ $\mathrm{Hz}, 1 \mathrm{H}), 5.35(\mathrm{~d}, J=6.9 \mathrm{~Hz}, 1 \mathrm{H}), 5.31(\mathrm{~d}, J=7.0 \mathrm{~Hz}, 1 \mathrm{H}), 4.80(\mathrm{~s}, 1 \mathrm{H}), 4.74(\mathrm{~d}, J=12.0 \mathrm{~Hz}, 1 \mathrm{H})$, $4.71(\mathrm{~d}, J=12.0 \mathrm{~Hz}, 1 \mathrm{H}), 3.69(\mathrm{~s}, 3 \mathrm{H}), 2.88(\mathrm{dd}, J=17.7,7.1 \mathrm{~Hz}, 1 \mathrm{H}), 2.69(\mathrm{dd}, J=7.0,2.5 \mathrm{~Hz}$, 1H), 2.66-2.51 (m, 2H), $2.35(\mathrm{~d}, J=17.8 \mathrm{~Hz}, 1 \mathrm{H}), 1.88(\mathrm{~s}, 3 \mathrm{H}), 1.62(\mathrm{~s}, 3 \mathrm{H}), 1.60-1.45(\mathrm{~m}, 2 \mathrm{H})$, $1.00(\mathrm{~s}, 3 \mathrm{H})$.

${ }^{13} \mathbf{C}\left\{{ }^{1} \mathbf{H}\right\}$ NMR $\left(126 \mathrm{MHz}, \mathrm{CDCl}_{3}\right) \delta 209.2,182.8,168.5,143.4,136.5,128.8,128.5,128.2$, $127.1,118.6,92.2,73.7,71.5,51.5,51.3,40.4,28.7,23.8,22.7,20.8,5.9$. 
HRMS (ESI/TOF) m/z: [M+Na $]^{+}$Calcd for $\mathrm{C}_{23} \mathrm{H}_{30} \mathrm{O}_{6} \mathrm{Na} 425.1935$; Found 425.1929.

Minor diastereomer:

$\mathbf{R}_{\boldsymbol{f}}=0.18$ (33\% EtOAc in hexanes), visualized by UV

${ }^{1}$ H NMR $\left(500 \mathrm{MHz}, \mathrm{CDCl}_{3}\right) \delta 7.38-7.28(\mathrm{~m}, 5 \mathrm{H}), 5.94(\mathrm{t}, J=7.3 \mathrm{~Hz}, 1 \mathrm{H}), 5.34(\mathrm{~s}, 2 \mathrm{H}), 4.74(\mathrm{~d}$, $J=11.8 \mathrm{~Hz}, 1 \mathrm{H}), 4.71(\mathrm{~d}, J=11.8 \mathrm{~Hz}, 1 \mathrm{H}), 4.37(\mathrm{~s}, 1 \mathrm{H}), 3.71(\mathrm{~s}, 3 \mathrm{H}), 2.86(\mathrm{ddd}, J=17.7,7.1,1.4$

$\mathrm{Hz}, 1 \mathrm{H}), 2.67(\mathrm{dd}, J=7.1,2.7 \mathrm{~Hz}, 1 \mathrm{H}), 2.63-2.45(\mathrm{~m}, 2 \mathrm{H}), 2.49(\mathrm{~d}, J=18.2 \mathrm{~Hz}, 1 \mathrm{H}), 1.87(\mathrm{~s}, 3 \mathrm{H})$, $1.62(\mathrm{~s}, 3 \mathrm{H}), 1.44-1.39(\mathrm{~m}, 2 \mathrm{H}), 1.16(\mathrm{~s}, 3 \mathrm{H})$.

${ }^{13} \mathbf{C}\left\{{ }^{1} \mathbf{H}\right\}$ NMR $\left(126 \mathrm{MHz}, \mathrm{CDCl}_{3}\right)$ 208.2, 182.6, 168.6, 143.5, 136.6, 128.8, 128.5, 128.2, 127.1, $118.8,92.2,73.9,71.5,54.3,51.4,36.1,28.6,25.2,23.9,20.8,5.9$.

HRMS (ESI/TOF) m/z: [M+Na $]^{+}$Calcd for $\mathrm{C}_{23} \mathrm{H}_{30} \mathrm{O}_{6} \mathrm{Na}$ 425.1935: Found 425.1916.

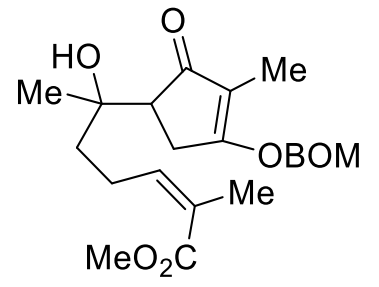

11

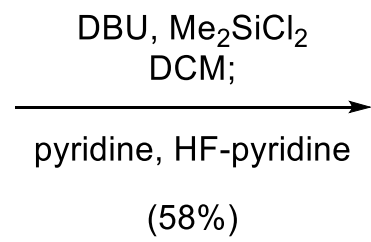

$(58 \%)$

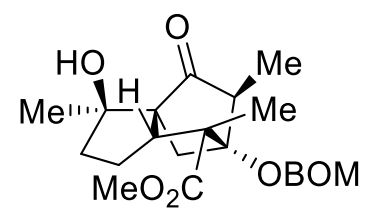

$( \pm)-13$

Diels-Alder adduct 13. Aldol 11 (diastereomeric mixture; $8.06 \mathrm{~g}, 20.0 \mathrm{mmol}$ ) was placed in a $500 \mathrm{~mL}$ round bottom flask equipped with a magnetic stir bar, and fitted with a rubber septum. The flask was evacuated, and then back-filled with argon. $\mathrm{CH}_{2} \mathrm{Cl}_{2}(180 \mathrm{~mL})$ was added, and the solution was cooled to $0{ }^{\circ} \mathrm{C}$. $\mathrm{DBU}(17.0 \mathrm{~mL}, 120 \mathrm{mmol})$ then $\mathrm{Me}_{2} \mathrm{SiCl}_{2}(5.0 \mathrm{~mL}, 40 \mathrm{mmol})$ were added to the flask. The rubber septum was then quickly exchanged for a greased glass stopper, and the neck of the flask was wrapped in Teflon tape, then parafilm. A Keck clamp was added to secure the stopper. After $30 \mathrm{~min}$ at $0{ }^{\circ} \mathrm{C}$, the solution was heated to $40{ }^{\circ} \mathrm{C}$ overnight (ca. $15 \mathrm{~h}$ ). The following morning, the reaction flask was allowed to cool to room temperature. The stopper was exchanged for a rubber septum. Pyridine $(9.4 \mathrm{~mL}, 0.12 \mathrm{~mol})$ then $70 \% \mathrm{HF}$ in pyridine $(2.32$ 
$\mathrm{mL}, 89 \mathrm{mmol})^{5}$ were added, and the reaction mixture was stirred for $1 \mathrm{~h}$. Saturated $\mathrm{NaHCO}_{3}$ (aq) $(50 \mathrm{~mL})$ was added slowly to the solution. Solid $\mathrm{NaHCO}_{3}$ was added in portions to the vigorously stirred reaction mixture until bubbling had ceased. The reaction mixture was then stirred for an additional $5 \mathrm{~min}$, then transferred to a separatory funnel and the organic layer was collected. The aqueous layer was washed with $\mathrm{CHCl}_{3}(2 \times 50 \mathrm{~mL})$. The combined organic layers were washed with saturated $\mathrm{CuSO}_{4}(\mathrm{aq})(150 \mathrm{~mL})$, which was back extracted with $\mathrm{CHCl}_{3}(50 \mathrm{~mL})$. The combined organic extracts were dried over $\mathrm{MgSO}_{4}$, and passed through a small plug of silica. The silica was washed with $\mathrm{Et}_{2} \mathrm{O}(150 \mathrm{~mL})$. The filtrate was concentrated in vacuo and purified by flash column chromatography on silica $(10-30 \%$ EtOAc in hexanes gradient), to afford 13 (4.65 $\mathrm{g}, 58 \%)$ as an orange oil.

$\mathbf{R}_{\boldsymbol{f}}=0.30$ (20\% EtOAc in hexanes), visualized by Hanessian's stain.

${ }^{1}$ H NMR $\left(500 \mathrm{MHz}, \mathrm{CDCl}_{3}\right) \delta 7.38-7.28(\mathrm{~m}, 5 \mathrm{H}), 5.06(\mathrm{~d}, J=7.7 \mathrm{~Hz}, 1 \mathrm{H}), 4.84(\mathrm{~d}, J=7.7 \mathrm{~Hz}$, 1H), $4.68(\mathrm{~d}, J=11.7 \mathrm{~Hz}, 1 \mathrm{H}), 4.62(\mathrm{~d}, J=11.7 \mathrm{~Hz}, 1 \mathrm{H}), 4.27(\mathrm{~s}, 1 \mathrm{H}), 3.71(\mathrm{~s}, 3 \mathrm{H}), 3.04(\mathrm{~d}, J=$ $10.0 \mathrm{~Hz}, 1 \mathrm{H}), 2.96(\mathrm{q}, J=7.1 \mathrm{~Hz}, 1 \mathrm{H}), 2.34(\mathrm{ddd}, J=10.8,6.0,2.1 \mathrm{~Hz}, 1 \mathrm{H}), 2.15-2.03(\mathrm{~m}, 1 \mathrm{H})$, $1.95(\mathrm{dd}, J=9.9,2.2 \mathrm{~Hz}, 1 \mathrm{H}), 1.87-1.79(\mathrm{~m}, 2 \mathrm{H}), 1.53-1.43(\mathrm{~m}, 1 \mathrm{H}), 1.43(\mathrm{~s}, 3 \mathrm{H}), 1.30(\mathrm{~d}, J=$ 7.1Hz, 3H), $1.30(\mathrm{~s}, 3 \mathrm{H})$.

${ }^{13} \mathbf{C}\left\{{ }^{1} \mathbf{H}\right\}$ NMR $\left(126 \mathrm{MHz}, \mathrm{CDCl}_{3}\right) 217.8,174.1,137.5,128.7,128.07,128.05,92.4,89.0,79.7$, $70.2,68.2,58.2,57.1,56.1,51.9,40.5,40.3,25.1,24.5,21.8,10.2$.

HRMS (ESI/TOF) m/z: [M+Na $]^{+}$Calcd for $\mathrm{C}_{23} \mathrm{H}_{30} \mathrm{O}_{6} \mathrm{Na}$ 425.1935: Found 425.1942.

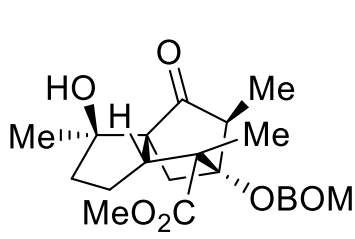

$( \pm)-13$
1. $\mathrm{LiAlH}_{4}, \mathrm{Et}_{2} \mathrm{O}$

2. TBSCl, imidazole DMAP, DMF

3. DMP, $\mathrm{CH}_{2} \mathrm{Cl}_{2}$ (65\%, 3 steps)

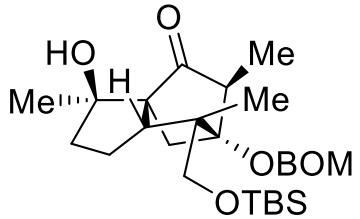

14 
Silyl ether 14. A suspension of $\mathrm{LiAlH}_{4}(3.44 \mathrm{~g}, 85.9 \mathrm{mmol})$ in diethyl ether $(100 \mathrm{~mL})$ was cooled to $0{ }^{\circ} \mathrm{C}$. A solution of ketone $13(8.64 \mathrm{~g}, 21.5 \mathrm{mmol})$ in diethyl ether $(100 \mathrm{~mL})$ was added via canula over the course of $10 \mathrm{~min}$. After $1 \mathrm{~h}$, saturated Rochelle's salt (aq) (20 mL) was added very slowly until the bubbling had ceased. Then an additional $280 \mathrm{~mL}$ of the Rochelle's salt solution was added, and the mixture was stirred vigorously for $2 \mathrm{~h}$. The mixture was transferred to a separatory funnel and the organic layer was collected. The aqueous layer was washed with diethyl ether $(2 \times 50 \mathrm{~mL})$. The combined organic layers were dried over $\mathrm{MgSO}_{4}$ and concentrated in vacuo to yield the crude triol which was used without further purification.

The crude triol, imidazole (14.6 g, $0.251 \mathrm{~mol})$, DMAP (2.63 g, $21.5 \mathrm{mmol})$, TBSCl (13.0 g, $86.2 \mathrm{mmol})$, and DMF (120 mL) were combined in a round bottom flask and stirred at RT for $4 \mathrm{~h}$. No attempt was made to exclude moisture or air from the reaction. The reaction mixture was transferred to a separatory funnel and diluted with saturated $\mathrm{NH}_{4} \mathrm{Cl}$ (aq) $(500 \mathrm{~mL})$. The aqueous layer was extracted with $\mathrm{CH}_{2} \mathrm{Cl}_{2}(3 \times 50 \mathrm{~mL})$. The combined organic layers were washed with $\mathrm{H}_{2} \mathrm{O}(2 \times 500 \mathrm{~mL})$, and the aqueous washes were back extracted with $\mathrm{CH}_{2} \mathrm{Cl}_{2}(2 \times 50 \mathrm{~mL})$. The combined organic layers were dried over $\mathrm{MgSO}_{4}$ and concentrated in vacuo to yield the monoprotected triol which was used without further purification.

To the crude product was added $\mathrm{NaHCO}_{3}(16 \mathrm{~g}, 0.19 \mathrm{~mol})$ and $\mathrm{CH}_{2} \mathrm{Cl}_{2}(200 \mathrm{~mL})$. No attempt was made to exclude moisture or air from the mixture. Dess-Martin periodinane (13.7 $\mathrm{g}, 32.3$ mmol) was added, and the reaction mixture was stirred at RT overnight (ca. $15 \mathrm{~h}$ ). The following morning, saturated $\mathrm{Na}_{2} \mathrm{~S}_{2} \mathrm{O}_{3}(\mathrm{aq})(300 \mathrm{~mL})$ was added and the mixture was stirred for $2 \mathrm{~h}$, before being transferred to a separatory funnel. The organic layer was collected. The aqueous layer was washed with $\mathrm{CHCl}_{3}(2 \times 50 \mathrm{~mL})$. The combined organic layers were dried over $\mathrm{MgSO}_{4}$ and passed through a small plug of silica. The silica was washed with ethyl acetate $(300 \mathrm{~mL})$. The filtrate 
was concentrated in vacuo and purified by flash column chromatography on silica $(0-20 \%$ EA in hexanes gradient), to afford $\mathbf{1 4}(6.81 \mathrm{~g}, 65 \%$ over 3 steps $)$ as a colorless oil which solidified to a white crystalline solid upon storage in the freezer.

$\mathbf{R}_{\boldsymbol{f}}=0.70$ (25\% EtOAc in hexanes), visualized by Hanessian's stain.

${ }^{1} \mathbf{H}$ NMR $\left(500 \mathrm{MHz}, \mathrm{CDCl}_{3}\right) \delta{ }^{1} \mathrm{H} \mathrm{NMR}\left(500 \mathrm{MHz}, \mathrm{CDCl}_{3}\right) \delta 7.39-7.28(\mathrm{~m}, 5 \mathrm{H}), 4.96(\mathrm{~d}, J=$ $7.5 \mathrm{~Hz}, 1 \mathrm{H}), 4.86(\mathrm{~d}, J=7.5 \mathrm{~Hz}, 1 \mathrm{H}), 4.71(\mathrm{~d}, J=11.7 \mathrm{~Hz}, 1 \mathrm{H}), 4.61(\mathrm{~d}, J=11.8 \mathrm{~Hz}, 1 \mathrm{H}), 4.42$ $(\mathrm{d}, J=2.0 \mathrm{~Hz}, 1 \mathrm{H}), 3.71(\mathrm{~d}, J=10.2 \mathrm{~Hz}, 1 \mathrm{H}), 3.57(\mathrm{~d}, J=10.3 \mathrm{~Hz}, 1 \mathrm{H}), 2.88(\mathrm{q}, J=7.1 \mathrm{~Hz}, 1 \mathrm{H})$, $2.51(\mathrm{~d}, J=9.5 \mathrm{~Hz}, 1 \mathrm{H}), 2.20(\mathrm{ddd}, J=9.9,7.5,2.2 \mathrm{~Hz}, 1 \mathrm{H}), 2.10-1.96(\mathrm{~m}, 2 \mathrm{H}), 1.86(\mathrm{ddd}, J=$ 12.7, 8.6, 3.8 Hz, 1H), $1.80(\mathrm{dd}, J=9.3,2.0 \mathrm{~Hz}, 1 \mathrm{H}), 1.78-1.72(\mathrm{~m}, 1 \mathrm{H}), 1.29(\mathrm{~s}, 3 \mathrm{H}), 1.27(\mathrm{~d}, J$ $=7.1 \mathrm{~Hz}, 3 \mathrm{H}), 1.03(\mathrm{~s}, 3 \mathrm{H}), 0.90(\mathrm{~s}, 9 \mathrm{H}), 0.07(\mathrm{~s}, 3 \mathrm{H}), 0.06(\mathrm{~s}, 3 \mathrm{H})$.

${ }^{13} \mathbf{C}\left\{{ }^{1} \mathbf{H}\right\}$ NMR $\left(126 \mathrm{MHz}, \mathrm{CDCl}_{3}\right)$ 219.1, 137.5, 128.7, 128.1, 127.9, 92.1, 87.8, 79.9, 70.2, 68.2, $66.9,56.5,55.2,49.3,40.7,39.8,26.0,24.8,23.7,22.4,18.2,10.3,-5.5,-5.6$.

HRMS (ESI/TOF) m/z: [M+Na] $]^{+}$Calcd for $\mathrm{C}_{28} \mathrm{H}_{44} \mathrm{O}_{5} \mathrm{SiNa} 511.2851$; Found 511.2834.

Melting Point: $85-88^{\circ} \mathrm{C}$

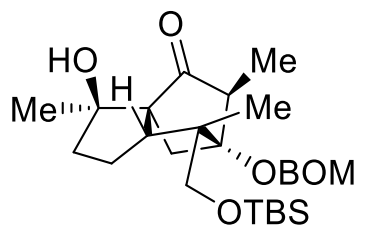

14

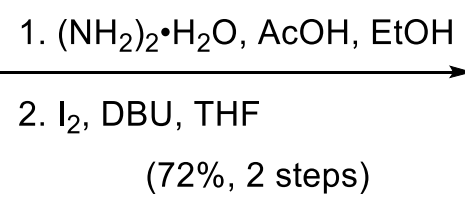

(72\%, 2 steps)

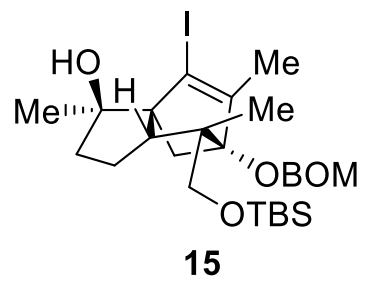

15

Vinyl Iodide 15. Ketone $14(6.35 \mathrm{~g}, 13.0 \mathrm{mmol}),\left(\mathrm{NH}_{2}\right)_{2} \cdot \mathrm{H}_{2} \mathrm{O}(19.7 \mathrm{~mL}, 0.402 \mathrm{~mol}), \mathrm{AcOH}$ (4.4 mL, $110 \mathrm{mmol})$, and EtOH (29 mL) were combined in a $100 \mathrm{~mL}, 1$-neck round bottom flask equipped with condenser. No attempt was made to exclude air or moisture. The mixture was heated to reflux for $4 \mathrm{~h}$, then allowed to cool to RT. The crude mixture was transferred to a 
separatory funnel along with $\mathrm{H}_{2} \mathrm{O}(300 \mathrm{~mL})$ and $\mathrm{Et}_{2} \mathrm{O}(75 \mathrm{~mL})$. The organic layer was collected. The aqueous layer was washed with additional $\mathrm{Et}_{2} \mathrm{O}(2 \times 75 \mathrm{~mL})$. The combined organic layers washed with brine $(50 \mathrm{~mL})$, dried over $\mathrm{MgSO}_{4}$, and passed through a small plug of silica. The silica was washed with ethyl acetate $(300 \mathrm{~mL})$. The filtrate was concentrated in vacuo and the hydrazone was used without further purification.

The crude hydrazone was azeotroped twice with dry benzene to get rid of any residual water, and then left under high vacuum for at least $30 \mathrm{~min}$. Next, the flask of crude hydrazone was placed under argon, and THF (150 mL) and DBU (18.9 mL, $0.127 \mathrm{~mol})$ were added. A solution of $\mathrm{I}_{2}$ (12.8 $\mathrm{g}, 50.4 \mathrm{mmol})$ in THF $(90 \mathrm{ml})$ was prepared in a separate flask. This solution was added to the reaction dropwise until the yellow color of iodine persisted for longer than 30 sec. $^{6}$ The reaction was then stirred for an additional 10 min. Saturated $\mathrm{Na}_{2} \mathrm{~S}_{2} \mathrm{O}_{3}(\mathrm{aq})(150 \mathrm{~mL})$ was added, the mixture was transferred to a separatory funnel and the mixture was washed with $\mathrm{Et}_{2} \mathrm{O}(3 \times 75$ $\mathrm{mL})$. The combined organic layers were washed with brine $(100 \mathrm{~mL})$, dried over $\mathrm{MgSO}_{4}$, and passed through a small plug of silica. The silica was washed with additional $\mathrm{Et}_{2} \mathrm{O}(200 \mathrm{~mL})$. The filtrate was concentrated in vacuo and purified by flash column chromatography on silica $(0-15 \%$ EtOAc in hexanes gradient), to afford $\mathbf{1 5}$ (5.62 g, 72\% over 2 steps) as a colorless oil.

$\mathbf{R}_{\boldsymbol{f}}=0.46$ (10\% EtOAc in hexanes), visualized by Hanessian's stain

${ }^{1} \mathbf{H}$ NMR $\left(500 \mathrm{MHz}, \mathrm{CDCl}_{3}\right) \delta 7.46-7.27(\mathrm{~m}, 5 \mathrm{H}), 4.80(\mathrm{~d}, J=6.9 \mathrm{~Hz}, 1 \mathrm{H}), 4.71(\mathrm{~d}, J=7.0 \mathrm{~Hz}$, $1 \mathrm{H}), 4.69(\mathrm{~d}, J=12.0 \mathrm{~Hz}, 1 \mathrm{H}), 4.57(\mathrm{~d}, J=12.0 \mathrm{~Hz}, 1 \mathrm{H}), 3.76(\mathrm{~d}, J=10.2 \mathrm{~Hz}, 1 \mathrm{H}), 3.73(\mathrm{~d}, J=$ $10.4 \mathrm{~Hz}, 1 \mathrm{H}), 2.49(\mathrm{~s}, 1 \mathrm{H}), 2.04-1.95(\mathrm{~m}, 2 \mathrm{H}), 1.93-1.77(\mathrm{~m}, 4 \mathrm{H}), 1.75(\mathrm{~s}, 3 \mathrm{H}), 1.73-1.65$ (m, 1H), $1.34(\mathrm{~s}, 3 \mathrm{H}), 0.90(\mathrm{~s}, 9 \mathrm{H}), 0.85(\mathrm{~s}, 3 \mathrm{H}), 0.064(\mathrm{~s}, 3 \mathrm{H}), 0.056(\mathrm{~s}, 3 \mathrm{H})$. ${ }^{13} \mathbf{C}\left\{{ }^{1} \mathbf{H}\right\}$ NMR $\left(126 \mathrm{MHz}, \mathrm{CDCl}_{3}\right) \delta 154.0,137.8,128.6,127.93,127.89,95.5,93.3,92.2,78.8$, $69.9,66.8,64.7,52.6,51.7,49.9,40.9,26.0,23.8,22.5,22.2,18.3,17.2,-5.4,-5.5$. 
HRMS (ESI/TOF) m/z: [M+Na] ${ }^{+}$Calcd for $\mathrm{C}_{28} \mathrm{H}_{43} \mathrm{IO}_{4} \mathrm{SiNa} 621.1868$; Found 621.1888.

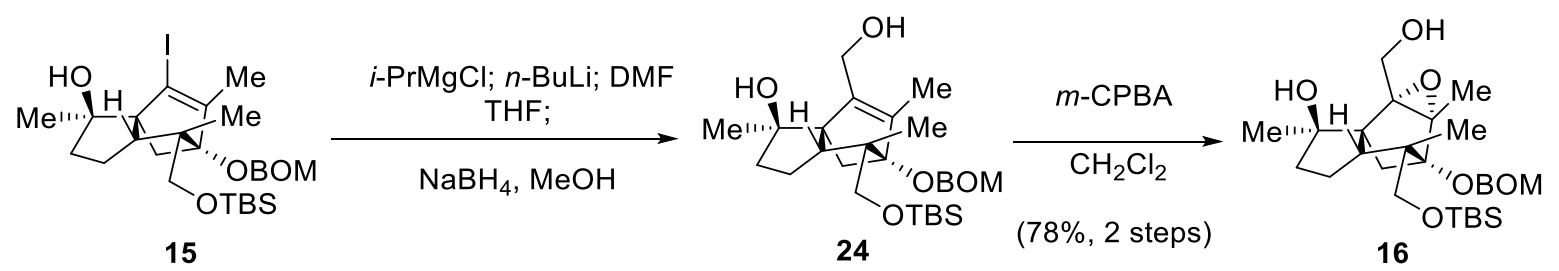

Oxirane 16. Vinyl iodide 15 (5.62 g, $9.38 \mathrm{mmol})$ was azeotroped twice with dry benzene, and then left under high vacuum for at least $30 \mathrm{~min}$. Next, THF $(30 \mathrm{~mL})$ was added and the flask was cooled to $0{ }^{\circ} \mathrm{C}$. $i$ - $\operatorname{PrMgCl}(2 \mathrm{M}$ in diethyl ether $)(9.20 \mathrm{~mL}, 18.4 \mathrm{mmol})$ was added. After $10 \mathrm{~min}$ the flask was cooled to $-78^{\circ} \mathrm{C}$, and $n$-BuLi (2.25 M in hexanes) (12.5 mL, 28.1 mmol) was added. After $20 \mathrm{~min}$, DMF (4.35 mL, $56.3 \mathrm{mmol})$ was added, and the flask was allowed to warm to RT over 60 min. $\mathrm{MeOH}(136 \mathrm{~mL})$, then $\mathrm{NaBH}_{4}(2.85 \mathrm{~g}, 75.1 \mathrm{mmol})$ were added. After $10 \mathrm{~min}$ saturated $\mathrm{NH}_{4} \mathrm{Cl}(\mathrm{aq})$ was added dropwise until bubbling had ceased. Additional saturated $\mathrm{NH}_{4} \mathrm{Cl}$ (aq) $(200 \mathrm{~mL})$ was added and the reaction mixture was transferred to a separatory funnel. The mixture was extracted with $\mathrm{CH}_{2} \mathrm{Cl}_{2}(3 \times 80 \mathrm{~mL})$, and the combined organic layers were dried over $\mathrm{MgSO}_{4}$ and concentrated in vacuo to yield the crude allylic alcohol which was used without further purification. The crude allylic alcohol could be purified on by flash column chromatography on silica $(0-100 \%$ EtOAc in hexanes gradient $)$.

$\mathrm{CH}_{2} \mathrm{Cl}_{2}(48 \mathrm{~mL})$ was added to the crude allylic alcohol. No attempt was made to exclude air or moisture. $m$-CPBA (ca. $70 \%$ with $\left.\mathrm{H}_{2} \mathrm{O}\right)$ (4.55 g, ca. $18.4 \mathrm{mmol}$ ) was added in two portions. After $30 \mathrm{~min}$, saturated $\mathrm{NaHCO}_{3}(75 \mathrm{~mL})$ and saturated $\mathrm{Na}_{2} \mathrm{~S}_{2} \mathrm{O}_{3}(75 \mathrm{~mL})$ were added, and the mixture was stirred for $1 \mathrm{~h}$. Next, the mixture was transferred to a separatory funnel and the organic layer was collected. The aqueous layer was washed with $\mathrm{CHCl}_{3}(2 \times 75 \mathrm{~mL})$. The combined organic layers were dried over $\mathrm{MgSO}_{4}$ and passed through a small plug of silica. The silica was washed with EtOAc $(200 \mathrm{~mL})$, concentrated in vacuo and purified by flash column 
chromatography on silica ( 0 - $100 \%$ EtOAc in hexanes gradient), to afford 16 (4.05 g, 83\% over 2 steps) as a foamy white solid.

\section{Epoxy Alcohol 16}

$\mathbf{R}_{\boldsymbol{f}}=0.42$ (40\% EtOAc in hexanes), visualized by Hanessian's stain

${ }^{1} \mathbf{H}$ NMR (499 MHz, $\left.\mathrm{CDCl}_{3}\right) \delta$ 7.39-7.33 (m, 4H), 7.32-7.33 (m, 1H), $4.82(\mathrm{~d}, J=6.7 \mathrm{~Hz}, 1 \mathrm{H})$, $4.78(\mathrm{~d}, J=6.7 \mathrm{~Hz}, 1 \mathrm{H}), 4.66(\mathrm{~d}, J=12.0 \mathrm{~Hz}, 1 \mathrm{H}), 4.59(\mathrm{~d}, J=12.0 \mathrm{~Hz}, 1 \mathrm{H}), 4.23(\mathrm{~d}, J=12.5$ $\mathrm{Hz}, 1 \mathrm{H}), 3.68(\mathrm{~d}, J=10.2 \mathrm{~Hz}, 1 \mathrm{H}), 3.62(\mathrm{~d}, J=10.2 \mathrm{~Hz}, 1 \mathrm{H}), 3.56(\mathrm{~d}, J=12.5 \mathrm{~Hz}, 1 \mathrm{H}), 2.13$ (ddd, $J=10.6,5.4,2.6 \mathrm{~Hz}, 1 \mathrm{H}), 2.02-1.93(\mathrm{~m}, 1 \mathrm{H}), 1.91-1.84(\mathrm{~m}, 3 \mathrm{H}), 1.80-1.74(\mathrm{~m}, 1 \mathrm{H}), 1.44$ (s, 3H), $1.37(\mathrm{~d}, J=9.5 \mathrm{~Hz}, 1 \mathrm{H}), 1.35(\mathrm{~s}, 3 \mathrm{H}), 1.01(\mathrm{~s}, 3 \mathrm{H}), 0.89(\mathrm{~s}, 9 \mathrm{H}), 0.05(\mathrm{~s}, 3 \mathrm{H}), 0.04(\mathrm{~s}$, $3 \mathrm{H})$.

${ }^{13} \mathbf{C}\left\{{ }^{1} \mathbf{H}\right\}$ NMR $\left(151 \mathrm{MHz}, \mathrm{CDCl}_{3}\right) 137.8,128.6,127.9,127.8,91.7,90.0,78.7,69.8,66.4,63.2$, $63.1,62.4,59.1,53.2,50.2,40.9,32.5,25.9,24.4,23.1,22.7,18.2,13.2,-5.44-5.55$

HRMS (ESI/TOF) m/z: [M+Na] ${ }^{+}$Calcd for $\mathrm{C}_{29} \mathrm{H}_{46} \mathrm{O}_{6} \mathrm{SiNa} 541.2956$; found 541.2935.

Melting Point: $100-102^{\circ} \mathrm{C}$

\section{Allylic Alcohol 24}

$\mathbf{R}_{\boldsymbol{f}}=0.28$ (30\% EtOAc in hexanes), visualized by Hanessian's stain

${ }^{1} \mathbf{H}$ NMR $\left(500 \mathrm{MHz}, \mathrm{CDCl}_{3}\right) \delta$ 7.37-7.27 (m, 5H), $4.81(\mathrm{~d}, J=6.9 \mathrm{~Hz}, 1 \mathrm{H}), 4.70(\mathrm{~d}, J=7.0 \mathrm{~Hz}$, $1 \mathrm{H}), 4.70(\mathrm{~d}, J=11.2 \mathrm{~Hz}, 1 \mathrm{H}), 4.56(\mathrm{~d}, J=12.0 \mathrm{~Hz}, 1 \mathrm{H}), 4.35(\mathrm{~d}, J=12.8 \mathrm{~Hz}, 1 \mathrm{H}), 4.22(\mathrm{~d}, J=$ $12.8 \mathrm{~Hz}, 1 \mathrm{H}), 3.78(\mathrm{~d}, J=10.0 \mathrm{~Hz}, 1 \mathrm{H}), 3.73(\mathrm{~d}, J=10.0 \mathrm{~Hz}, 1 \mathrm{H}), 3.52$ (br. s, $1 \mathrm{H}), 2.53$ (br. s, 1H), 2.03-1.88 (m, 3H), 1.86-1.78 (m, 3H), $1.71(\mathrm{~s}, 3 \mathrm{H}), 1.63(\mathrm{dd}, J=7.9,2.2 \mathrm{~Hz}, 1 \mathrm{H}), 1.36(\mathrm{~s}$, 3H), $0.90(\mathrm{~s}, J=2.7 \mathrm{~Hz}, 9 \mathrm{H}), 0.88(\mathrm{~s}, 3 \mathrm{H}), 0.06(\mathrm{~s}, 3 \mathrm{H}), 0.05(\mathrm{~s}, 3 \mathrm{H})$.

${ }^{13} \mathbf{C}\left\{{ }^{1} \mathbf{H}\right\}$ NMR $\left(126 \mathrm{MHz}, \mathrm{CDCl}_{3}\right) \delta 142.1,140.3,138.0,128.6,127.9,127.9,93.8,92.1,79.4$, $69.7,67.4,64.3,57.6,54.5,50.4,50.3,42.7,26.0,25.0,23.1,22.1,18.3,11.5,-5.37,-5.44$. 
HRMS (ESI/TOF) m/z: [M+Na] ${ }^{+}$Calcd for $\mathrm{C}_{29} \mathrm{H}_{46} \mathrm{O}_{5} \mathrm{SiNa}$ 525.3007; found 525.3006.

Melting Point: $122-126^{\circ} \mathrm{C}$

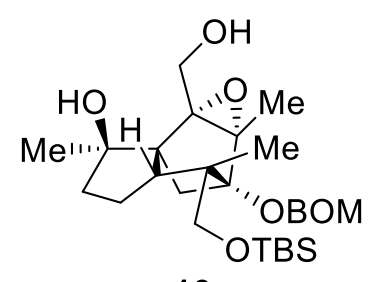

16

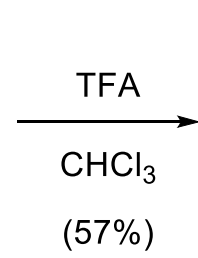

$(57 \%)$

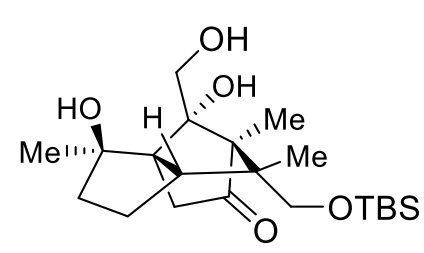

17

Cyclopentanone triol 17. A solution of TFA $(530 \mu \mathrm{L}, 6.93 \mathrm{mmol})$ in $\mathrm{CHCl}_{3}(41 \mathrm{~mL})$ was added to epoxide 16 (4.05 g, $7.80 \mathrm{mmol})$. The solution was stirred for $40 \mathrm{~h}$. No attempt was made to exclude moisture or air. $\mathrm{Et}_{3} \mathrm{~N}(1.71 \mathrm{~mL}, 12.3 \mathrm{mmol})$ was added, and the solution was concentrated in vacuo. The crude residue was purified by flash column chromatography on silica $(0-100 \%$ EtOAc in hexanes gradient $)$, to afford $17(1.77 \mathrm{~g}, 57 \%)$ as a white solid.

$\mathbf{R}_{\boldsymbol{f}}=0.21$ (40\% EtOAc in hexanes), visualized by Hanessian's stain

${ }^{1} \mathbf{H}$ NMR $\left(500 \mathrm{MHz}, \mathrm{CDCl}_{3}\right)^{7} \delta 4.38(\mathrm{dd}, J=11.4,3.0 \mathrm{~Hz}, 1 \mathrm{H}), 4.29(\mathrm{dd}, J=9.9,3.2 \mathrm{~Hz}, 1 \mathrm{H})$, 3.34 (app. t, $J=10.6 \mathrm{~Hz}, 1 \mathrm{H}), 3.28(\mathrm{~s}, 1 \mathrm{H}), 3.21(\mathrm{~d}, J=10.1 \mathrm{~Hz}, 1 \mathrm{H}), 3.12(\mathrm{~d}, J=10.1 \mathrm{~Hz}, 1 \mathrm{H})$, $2.91(\mathrm{ddd}, J=11.9,8.2,1.5 \mathrm{~Hz}, 1 \mathrm{H}), 2.72(\mathrm{~s}, 1 \mathrm{H}), 2.51(\mathrm{dd}, J=17.5,1.7 \mathrm{~Hz}, 1 \mathrm{H}), 2.13(\mathrm{ddd}, J=$ 14.6, 11.2, 3.2 Hz, 1H), 2.09 (d, $J=17.5 \mathrm{~Hz}, 1 \mathrm{H}), 1.96(\mathrm{ddd}, J=14.9,9.1,5.8 \mathrm{~Hz}, 1 \mathrm{H}), 1.83-$ $1.75(\mathrm{~m}, 1 \mathrm{H}), 1.51(\mathrm{ddd}, J=24.4,12.1,5.8 \mathrm{~Hz}, 1 \mathrm{H}), 1.34(\mathrm{~s}, 3 \mathrm{H}), 1.00(\mathrm{~s}, 3 \mathrm{H}), 0.88(\mathrm{~s}, 3 \mathrm{H}), 0.85$ (s, 9H), $-0.03(\mathrm{~s}, 3 \mathrm{H}),-0.04(\mathrm{~s}, 3 \mathrm{H})$.

${ }^{13} \mathbf{C}\left\{{ }^{1} \mathbf{H}\right\}$ NMR $\left(126 \mathrm{MHz}, \mathrm{CDCl}_{3}\right) \delta 213.6,84.1,76.6,69.8,66.0,63.2,60.6,51.6,45.1,39.5$, $39.4,25.9,25.0,23.5,19.6,18.2,6.4,-5.6,-5.7$.

HRMS (ESI/TOF) m/z: [M+Na] ${ }^{+}$Calcd for $\mathrm{C}_{21} \mathrm{H}_{38} \mathrm{O}_{5} \mathrm{SiNa} 421.2381$; found 421.2363.

Melting Point: $142-146^{\circ} \mathrm{C}$ 


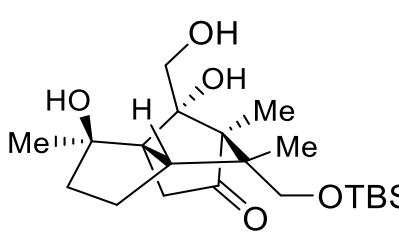

17

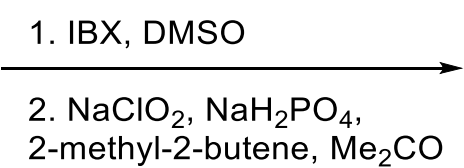

2-methyl-2-butene, $\mathrm{Me}_{2} \mathrm{CO}$

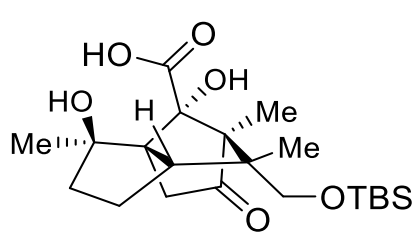

s7

Carboxylic acid S7. Alcohol 17 (0.64 g, $1.6 \mathrm{mmol})$ was dissolved in dry DMSO (11 mL). IBX (2.56 g, $9.15 \mathrm{mmol})$ was added and the reaction was stirred at RT overnight (ca. $15 \mathrm{~h})$. The following morning, $\mathrm{H}_{2} \mathrm{O}(30 \mathrm{~mL})$ was added, generating a white precipitate. The mixture was filtered, and the precipitate was washed with $\mathrm{CH}_{2} \mathrm{Cl}_{2}(30 \mathrm{~mL})$. The filtrate was transferred to a separatory funnel, and the organic layer was collected. The aqueous layer was extracted with $\mathrm{CH}_{2} \mathrm{Cl}_{2}(2 \times 15 \mathrm{~mL})$. The combined organic layers were washed with $\mathrm{H}_{2} \mathrm{O}(50 \mathrm{~mL})$ then saturated $\mathrm{NaHCO}_{3} / \mathrm{Na}_{2} \mathrm{~S}_{2} \mathrm{O}_{3}(\mathrm{aq})(50 \mathrm{~mL})$. Each aqueous layer was extracted with additional $\mathrm{CH}_{2} \mathrm{Cl}_{2}(2 \mathrm{x}$ $15 \mathrm{~mL}$ ). The combined organic layers were dried over $\mathrm{Na}_{2} \mathrm{SO}_{4}$, concentrated in vacuo, and carried on to the next step without additional purification.

To the crude aldehyde 22 was added acetone $(7 \mathrm{~mL}), \mathrm{H}_{2} \mathrm{O}(9 \mathrm{~mL}), 2$-methyl-2-butene (3.32 $\mathrm{mL}, 31.4 \mathrm{mmol})$, and $2 \mathrm{M} \mathrm{NaH}_{2} \mathrm{PO}_{4}(\mathrm{aq})(8.83 \mathrm{~mL}, 17.7 \mathrm{mmol})$. To the mixture was added solid $\mathrm{NaClO}_{2}(80 \%$ technical grade $)(2.19 \mathrm{~g}, 17.6 \mathrm{mmol})$. No attempt was made to exclude air from the reaction. The mixture was stirred vigorously for $45 \mathrm{~min}$, then $\mathrm{pH} 2.5$ buffer $(30 \mathrm{~mL})$ was added and the mixture was transferred to a separatory funnel. ${ }^{8}$ The mixture was extracted with EtOAc ( $5 \times 15 \mathrm{~mL})$. The combined organic extracts were dried over $\mathrm{Na}_{2} \mathrm{SO}_{4}$, concentrated in vacuo, and purified by flash column chromatography on silica $\left(0-10 \% \mathrm{MeOH}\right.$ :formic acid (20:1) in $\mathrm{CH}_{2} \mathrm{Cl}_{2}$ gradient), to afford impure acid $\mathbf{S} 7(580 \mathrm{mg})$ as a white foamy solid. The product was taken on to the next step without further purification.

The product could be purified by further chromatography, and characterization of the resulting 
white solid product is given below.

$\mathbf{R}_{f}=0.27\left(90 \% \mathrm{CH}_{2} \mathrm{Cl}_{2}: 9 \% \mathrm{MeOH}: 1 \%\right.$ formic acid), visualized by Hanessian's stain

${ }^{1} \mathbf{H}$ NMR (500 MHz, CD 3 OD) $\delta 3.28(\mathrm{~d}, J=10.2 \mathrm{~Hz}, 1 \mathrm{H}), 3.23(\mathrm{~d}, J=10.3 \mathrm{~Hz}, 1 \mathrm{H}), 3.13$ (app.

td, $J=10.0,1.6 \mathrm{~Hz}, 1 \mathrm{H}), 2.55(\mathrm{dd}, J=17.8,1.5 \mathrm{~Hz}, 1 \mathrm{H}), 2.28(\mathrm{~d}, J=17.8 \mathrm{~Hz}, 1 \mathrm{H}), 2.15-2.00$

(m, 2H), 1.76 (app. dtd, $J=12.8,8.6,4.4 \mathrm{~Hz}, 1 \mathrm{H}), 1.64$ (app. qd, $J=12.0,6.0 \mathrm{~Hz}, 1 \mathrm{H}), 1.37$ (s,

3H), $1.10(\mathrm{~s}, 3 \mathrm{H}), 1.08(\mathrm{~s}, 3 \mathrm{H}), 0.89$ (s, 9H), $0.014(\mathrm{~s}, 3 \mathrm{H}), 0.010(\mathrm{~s}, 3 \mathrm{H})$.

${ }^{13} \mathbf{C}\left\{{ }^{1} \mathbf{H}\right\}$ NMR $\left(151 \mathrm{MHz}, \mathrm{CD}_{3} \mathrm{OD}\right) \delta 214.6,176.0,86.4,77.3,74.3,67.8,63.9,52.9,45.2,41.5$, $41.0,26.3,24.2,22.6,19.8,19.0,8.2,-5.7^{9}$.

HRMS (ESI/TOF) m/z: [M-H] $]^{-}$Calcd for $\mathrm{C}_{21} \mathrm{H}_{35} \mathrm{O}_{6} \mathrm{Si} 411.2208$; found 411.2213.

Melting Point: $136-140{ }^{\circ} \mathrm{C}$

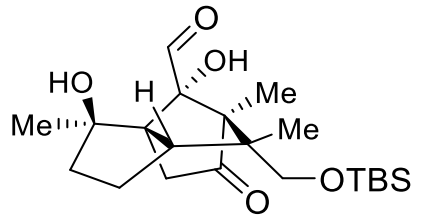

22

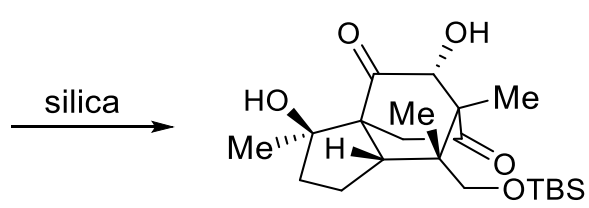

23

Tricycle 23: When crude aldehyde 22 was subjected to flash column chromatography on silica $(0-100 \%$ ethyl acetate in hexanes gradient $)$, the aldehyde was not recovered. Instead the major product is tricycle 23, which was isolated along with some impurities. As the product was not on the path to Illisimonin A 1, we did not attempt further purification.

$\mathbf{R}_{\boldsymbol{f}}=0.30$ (50\% ethyl acetate in hexanes), visualized by Hanessian's stain

${ }^{1}$ H NMR see below

${ }^{13} \mathbf{C}\left\{{ }^{1} \mathbf{H}\right\}$ NMR see below

HRMS (ESI/TOF) m/z: [M+Na] ${ }^{+}$Calcd for $\mathrm{C}_{21} \mathrm{H}_{36} \mathrm{O}_{5} \mathrm{SiNa} 419.2225$; found 419.2244. 


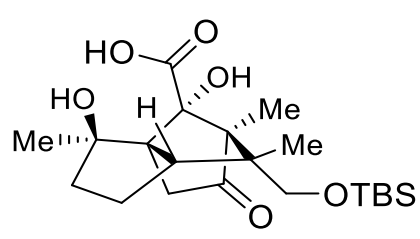

S7

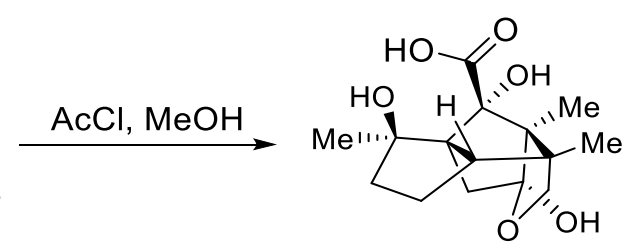

18

Lactol 18: $\mathrm{AcCl}(1.20 \mathrm{~mL}, 16.8 \mathrm{mmol})$ was added slowly to $\mathrm{MeOH}(28.8 \mathrm{~mL})$. When the solution had cooled to room temperature, it was added to impure acid S7 (580 mg), and the solution was stirred at room temperature for 4.5 hours. No attempt was made to exclude air or moisture from this reaction. The solvent was removed in vacuo, and the crude residue was purified by flash column chromatography on silica $\left(0-20 \% \mathrm{MeOH}\right.$ :formic acid (20:1) in $\mathrm{CH}_{2} \mathrm{Cl}_{2}$ gradient), to afford lactol 18 (196 mg, 41\% over 3 steps) as a white foamy solid.

$\mathbf{R}_{\boldsymbol{f}}=0.42\left(84 \% \mathrm{CH}_{2} \mathrm{Cl}_{2}: 15 \% \mathrm{MeOH}: 1 \%\right.$ formic acid $)$, visualized by Hanessian's stain ${ }^{1}$ H NMR (500 MHz, CD 3 OD) $\delta 3.65$ (d, $\left.J=8.6 \mathrm{~Hz}, 1 \mathrm{H}\right), 3.48$ (d, $\left.J=8.6 \mathrm{~Hz}, 1 \mathrm{H}\right), 3.08$ (app. td, $J=9.3,1.4 \mathrm{~Hz}, 1 \mathrm{H}), 2.41(\mathrm{dd}, J=12.8,1.6 \mathrm{~Hz}, 1 \mathrm{H}), 2.08(\mathrm{ddd}, J=14.3,11.1,3.4 \mathrm{~Hz}, 1 \mathrm{H}), 2.00$ (ddd, $J=14.8,11.1,3.4 \mathrm{~Hz}, 1 \mathrm{H}), 1.78(\mathrm{~d}, J=12.9 \mathrm{~Hz}, 1 \mathrm{H}), 1.66-1.59(\mathrm{~m}, 1 \mathrm{H}), 1.59-1.50$ (m, 1H), $1.31(\mathrm{~s}, 3 \mathrm{H}), 1.08(\mathrm{~s}, 3 \mathrm{H}), 1.00(\mathrm{~s}, 3 \mathrm{H})$.

${ }^{13} \mathbf{C}\left\{{ }^{1} \mathbf{H}\right\}$ NMR $\left(151 \mathrm{MHz}, \mathrm{CD}_{3} \mathrm{OD}\right) \delta 176.0,111.8,86.6,77.0,70.5,66.2,64.0,54.1,45.4,45.1$, $44.5,24.6,20.7,19.4,7.0$.

HRMS (ESI/TOF) m/z: [M+Na $]^{+}$Calcd for $\mathrm{C}_{15} \mathrm{H}_{22} \mathrm{O}_{6} \mathrm{Na} 321.1309$; found 321.1312.

Melting Point: $175-180{ }^{\circ} \mathrm{C}$

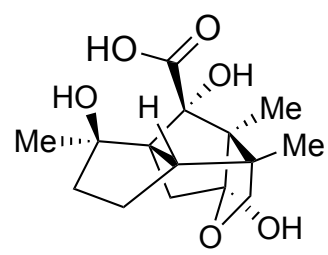

18

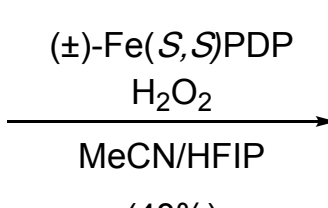

$(48 \%)$

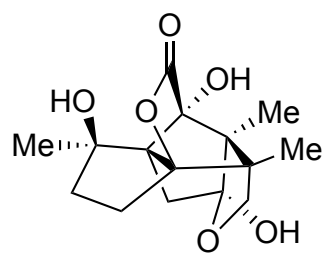

$( \pm)-1$

Illisimonin A (1): Lactol 18 (196 mg, 0.657 mmol) was azeotroped in HFIP:MeCN (3:1) three 
times to remove any traces of methanol. HFIP $(2.84 \mathrm{~mL})$ was added to the flask containing lactol 18 and a stir bar. The flask was left open to air.

Approximately racemic White's catalyst was prepared by mixing equal amounts of $\mathrm{Fe}(R, R) \operatorname{PDP}$ and $\mathrm{Fe}(S, S) \mathrm{PDP}$.

The following solutions were made open to air:

$5.52 \mathrm{~mL} \mathrm{MeCN}+200 \mu \mathrm{L} \mathrm{H}_{2} \mathrm{O}_{2} 30 \%$ w/w in $\mathrm{H}_{2} \mathrm{O}$

4.77 mL MeCN + 169.2 mg Fe(PDP)

$4.32 \mathrm{~mL}$ of the $\mathrm{H}_{2} \mathrm{O}_{2}$ solution (ca. $1.48 \mathrm{mmol}$ ) and $4.32 \mathrm{~mL}$ of the $\mathrm{Fe}(\mathrm{PDP})$ solution (ca. 0.164 mmol) were added dropwise, concurrently via syringe pump to the stirred solution of lactol 18 over 45 min. ${ }^{10}$ After this period, isopropanol $(300 \mu \mathrm{L}, 3.92 \mathrm{mmol})$ was added, and the crude reaction mixture was concentrated onto silica gel, and purified by flash column chromatography on silica $(0-50 \%$ acetone in hexanes gradient then $50 \%$ acetone in hexanes isocratic), to afford natural product $\mathbf{1}(93.9 \mathrm{mg}, 48 \%)$ as a white solid.

The same reaction was performed starting from lactol 18 (107.6 $\mathrm{mg}, 0.3607 \mathrm{mmol})$, and yielded natural product $1(50.2 \mathrm{mg}, 47 \%)$.

$\mathbf{R}_{\boldsymbol{f}}=0.32\left(10 \% \mathrm{MeOH}\right.$ in $\left.\mathrm{CH}_{2} \mathrm{Cl}_{2}\right)$, visualized by Hanessian's stain

$\mathbf{R}_{\boldsymbol{f}}=0.34$ (60\% acetone in hexanes), visualized by Hanessian's stain

${ }^{1}$ H NMR see below

${ }^{13} \mathbf{C}\left\{{ }^{1} \mathbf{H}\right\}$ NMR see below

HRMS (ESI/TOF) m/z: [M+Na $]^{+}$Calcd for $\mathrm{C}_{15} \mathrm{H}_{20} \mathrm{O}_{6} \mathrm{Na} 319.1153$; found 319.1164 . 
Melting Point: Illisimonin A 1 turned from a white solid to a yellowish-brown solid around 165 ${ }^{\circ} \mathrm{C}$, then liquefied between $169-175^{\circ} \mathrm{C}$

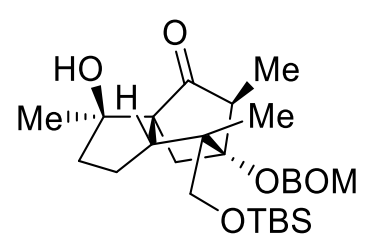

14

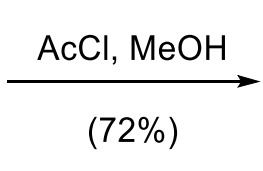

$(72 \%)$

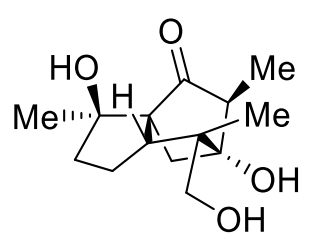

S8

Triol Ketone S8. Protected triol $14(30.3 \mathrm{mg}, 0.0620 \mathrm{mmol})$ was dissolved in $\mathrm{MeOH}(0.5 \mathrm{~mL})$. The solution was cooled to $0{ }^{\circ} \mathrm{C}$. Acetyl chloride $(0.1 \mathrm{~mL})$ was added dropwise, and the reaction mixture was stirred for $30 \mathrm{~min}$. Saturated $\mathrm{NaHCO}_{3}$ (aq) (1 mL) was added slowly. The reaction mixture was transferred to a separatory funnel, and washed with $\mathrm{Et}_{2} \mathrm{O}(3 \times 1 \mathrm{~mL})$. The organic layers were combined, dried over $\mathrm{MgSO}_{4}$ and concentrated in vacuo. The crude residue was purified by flash column chromatography on silica $(0-100 \%$ EtOAc in hexanes gradient), to afford ketone S8 (11.4 mg, 72\%) as a white solid.

$\mathbf{R}_{\boldsymbol{f}}=0.56(100 \%$ EA), visualized by Hanessian's stain

${ }^{1} \mathbf{H}$ NMR $\left(500 \mathrm{MHz}, \mathrm{CDCl}_{3}\right) \delta 4.50(\mathrm{~s}, 1 \mathrm{H}), 4.42(\mathrm{~d}, J=1.8 \mathrm{~Hz}, 1 \mathrm{H}), 4.12(\mathrm{dd}, J=10.5,3.8 \mathrm{~Hz}$ $1 \mathrm{H}), 3.59(\mathrm{dd}, J=10.5,4.5 \mathrm{~Hz}, 1 \mathrm{H}), 2.71(\mathrm{t}, J=4.5 \mathrm{~Hz}, 1 \mathrm{H}), 2.64$ (q, $J=7.2 \mathrm{~Hz}, 1 \mathrm{H}), 2.31$ (ddd, $J=10.3,6.3,1.7 \mathrm{~Hz}, 1 \mathrm{H}), 2.22(\mathrm{~d}, J=10.1 \mathrm{~Hz}, 1 \mathrm{H}), 1.97-1.81(\mathrm{~m}, 2 \mathrm{H}), 1.79-1.69(\mathrm{~m}, 1 \mathrm{H})$ $1.64-1.58(\mathrm{~m}, 1 \mathrm{H}), 1.58(\mathrm{dd}, J=10.1,1.8 \mathrm{~Hz}, 1 \mathrm{H}), 1.29(\mathrm{~s}, 3 \mathrm{H}), 1.28-1.23(\mathrm{~m}, 6 \mathrm{H})$. ${ }^{13} \mathbf{C}\left\{{ }^{1} \mathbf{H}\right\} \mathbf{N M R}\left(126 \mathrm{MHz}, \mathrm{CDCl}_{3}\right)$ 218.7, 85.5, 79.4, 71.4, 68.0, 58.1, 55.7, 44.6, 44.2, 40.9, $24.6,22.8,20.9,10.1$.

HRMS (ESI/TOF) m/z: $[\mathrm{M}+\mathrm{Na}]^{+}$Calcd for $\mathrm{C}_{14} \mathrm{H}_{22} \mathrm{O}_{4} \mathrm{Na} 277.1411$; Found 277.1414.

Melting Point: $138-140{ }^{\circ} \mathrm{C}$ 


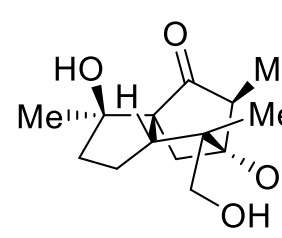

58
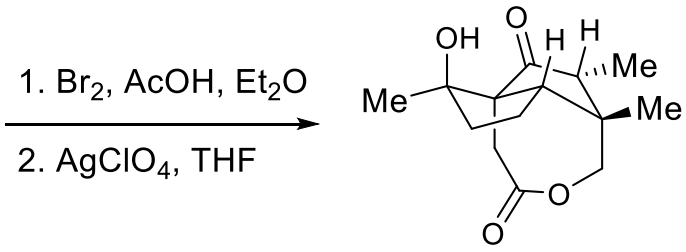

21

Lactone 21. Triol S8 $(11.4 \mathrm{mg}, 0.0449 \mathrm{mmol})$ was dissolved in $\mathrm{Et}_{2} \mathrm{O}(50 \mu \mathrm{L})$ and THF (150 $\mu \mathrm{L}$ ) and cooled to $0{ }^{\circ} \mathrm{C}$. No attempt was made to exclude air or moisture. A solution of bromine (100 $\mu \mathrm{L}, 1.88 \mathrm{mmol})$, acetic acid $(200 \mu \mathrm{L}, 3.49 \mathrm{mmol})$, THF $(2 \mathrm{~mL})$, and $\mathrm{Et}_{2} \mathrm{O}(2 \mathrm{~mL})$ was made. $100 \mu \mathrm{L}$ of this solution (ca. $4.35 \mu \mathrm{L}, 0.0783 \mathrm{mmol} \mathrm{Br}_{2}$ ) was added to the chilled solution of triol S8, and the reaction mixture was stirred for 20 min. Saturated $\mathrm{NaHCO}_{3}(\mathrm{aq})(0.5 \mathrm{~mL})$ and saturated $\mathrm{Na}_{2} \mathrm{~S}_{2} \mathrm{O}_{3}(0.5 \mathrm{~mL})$ was added. The mixture was transferred to a separatory funnel, and extracted with EtOAc $(3 \times 1 \mathrm{~mL})$. The combined organic layers were dried over $\mathrm{MgSO}_{4}$ and concentrated in vacuo to yield the crude bromide 19, which was used without further purification.

A suspension of $\mathrm{AgClO}_{4}(22 \mathrm{mg}, 0.106 \mathrm{mmol})$ in THF $(0.4 \mathrm{~mL})$ was made under an atmosphere of argon. A solution of the crude bromide 19 in THF $(0.1 \mathrm{~mL})$ was added. After 20 min saturated $\mathrm{NaHCO}_{3}(1 \mathrm{~mL})$ and EA $(1 \mathrm{~mL})$ were added. The crude mixture was filtered through a cotton plug. The filtrate was transferred to a separatory funnel and the organic layer was collected. The aqueous layer was extracted with additional EA $(2 \times 2 \mathrm{~mL})$. The combined organic layers were dried over $\mathrm{MgSO}_{4}$ and concentrated in vacuo. The crude mixture was purified by flash column chromatography on silica (60\% EtOAc in hexanes) to yield 21 as a white solid $(6.1 \mathrm{mg}, 54 \%$ over 2 steps)

$\mathbf{R}_{\boldsymbol{f}}=0.43$ (80\% EtOAc in hexanes), visualized by Hanessian's stain

Melting Point: $170-175{ }^{\circ} \mathrm{C}$ 
${ }^{1} \mathbf{H}$ NMR $\left(500 \mathrm{MHz}, \mathrm{CDCl}_{3}\right) \delta 4.42(\mathrm{~d}, J=13.4 \mathrm{~Hz}, 1 \mathrm{H}), 3.98(\mathrm{dd}, J=13.4,1.3 \mathrm{~Hz}, 1 \mathrm{H}), 3.10$ $(\mathrm{dd}, J=12.9,6.9 \mathrm{~Hz}, 1 \mathrm{H}), 2.76(\mathrm{dd}, J=15.0,0.9 \mathrm{~Hz}, 1 \mathrm{H}), 2.63(\mathrm{~d}, J=14.9 \mathrm{~Hz}, 1 \mathrm{H}), 2.44(\mathrm{q}, J=$ $7.3 \mathrm{~Hz}, 1 \mathrm{H}), 2.21(\mathrm{ddd}, J=15.3,9.1,6.5 \mathrm{~Hz}, 1 \mathrm{H}), 2.08-2.02(\mathrm{~m}, 1 \mathrm{H}), 1.79-1.73(\mathrm{~m}, 1 \mathrm{H}), 1.74$ (s, 1H), 1.66 (app. tdd, $J=12.5,10.9,6.4 \mathrm{~Hz}, 1 \mathrm{H}), 1.49$ (s, 3H), $1.22(\mathrm{~s}, 3 \mathrm{H}), 1.14(\mathrm{~s}, 3 \mathrm{H})$. ${ }^{13} \mathbf{C}\left\{{ }^{1} \mathbf{H}\right\}$ NMR $\left(126 \mathrm{MHz}, \mathrm{CDCl}_{3}\right)$ 213.6, 171.0, 77.0, 69.0, 64.2, 62.1, 54.7, 42.6, 40.6, 35.1, $24.7,23.1,16.4,8.8$.

HRMS (ESI/TOF) m/z: $[\mathrm{M}+\mathrm{Na}]^{+}$Calcd for $\mathrm{C}_{14} \mathrm{H}_{20} \mathrm{O}_{4} \mathrm{Na} 275.1254$; Found 275.1258. 


\section{Enantioenriched Section- Compounds reported in this section are enantioenriched}

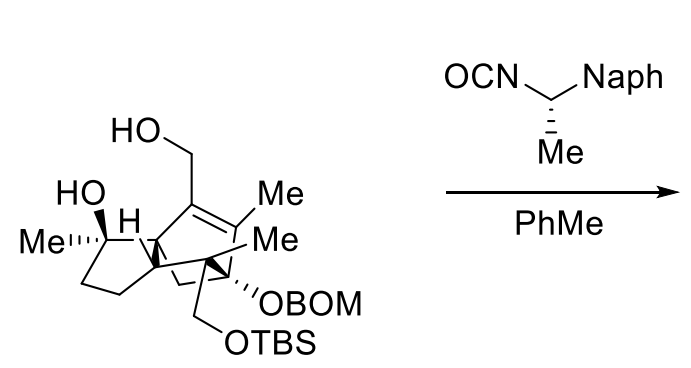

$( \pm)-24$

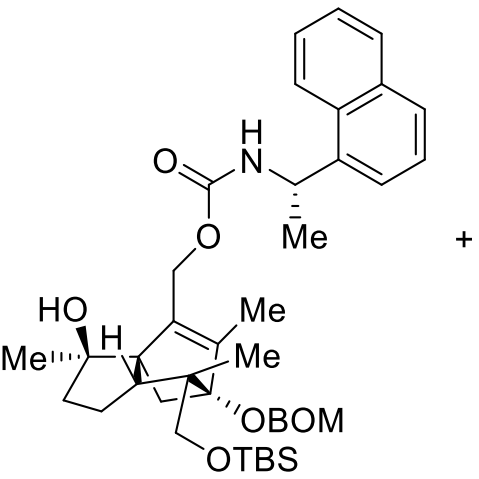

$(-)-26$

$39 \%, 91 \%$ ee

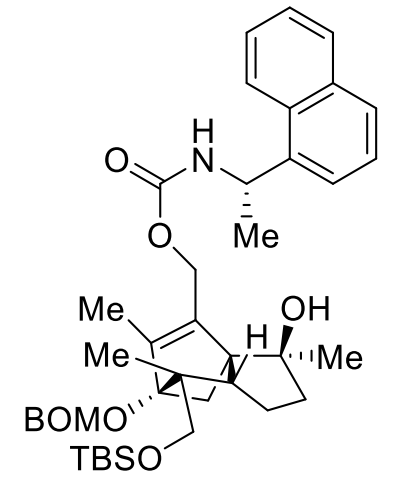

$(+)-27$

$44 \%, 91 \%$ ee

Carbamates (-)-26 and (+)-27. Allylic alcohol ( $( \pm)-24$ (340 mg, 0.676 mmol), DMAP (5 mg, 0.04 mmol), (S)-1-(1-isocyanatoethyl)naphthalene ${ }^{11}$ (146 mg, $\left.0.745 \mathrm{mmol}\right)$, and dry PhMe (1.6 mL) were combined in a sealed tube and heated to $110^{\circ} \mathrm{C}$ for $6 \mathrm{~h}$, then allowed to cool to RT. The solvent was removed in vacuo, and the mixture of diastereomers was isolated by flash column chromatography on silica $(10-30 \%$ EtOAc in hexanes $)$.

$\mathbf{R}_{\boldsymbol{f}}=0.47$ (30\% EtOAc in hexanes), visualized by UV

The mixture of diastereomers were partially separated by additional flash column chromatography on silica $\left(0-10 \% \mathrm{Et}_{2} \mathrm{O}\right.$ in $\left.\mathrm{CH}_{2} \mathrm{Cl}_{2}\right)$. The mixed fractions were resubjected to the same chromatography conditions two additional times. Carbamate (+)-27 (208.8 mg, 44\%) was isolated as a white foamy solid. Carbamate (-)-26 (186.4 mg, 39\%) was isolated as a white foamy solid. Additionally a mixture of the two diastereomers (18.4 mg, 4\%) was recovered.

\section{Carbamate (-)-26}

$\mathbf{R}_{f}=0.43\left(10 \% \mathrm{Et}_{2} \mathrm{O}\right.$ in $\left.\mathrm{CH}_{2} \mathrm{Cl}_{2}\right)$, visualized by $\mathrm{UV}$

${ }^{1} \mathbf{H}$ NMR $\left(600 \mathrm{MHz}, \mathrm{CDCl}_{3}\right)^{12} 8.09(\mathrm{~d}, J=8.3 \mathrm{~Hz}, 1 \mathrm{H}), 7.86(\mathrm{~d}, J=8.1 \mathrm{~Hz}, 1 \mathrm{H}), 7.79(\mathrm{~d}, J=8.0$ $\mathrm{Hz}, 1 \mathrm{H}), 7.55(\mathrm{t}, J=7.5 \mathrm{~Hz}, 1 \mathrm{H}), 7.52-7.47(\mathrm{~m}, J=7.0 \mathrm{~Hz}, 2 \mathrm{H}), 7.44(\mathrm{t}, J=7.5 \mathrm{~Hz}, 1 \mathrm{H}), 7.38-$ 
$7.27(\mathrm{~m}, 5 \mathrm{H}), 5.69-5.61(\mathrm{~m}, 1 \mathrm{H}), 5.00(\mathrm{~d}, J=7.7 \mathrm{~Hz}, 1 \mathrm{H}), 4.91(\mathrm{~d}, J=12.3 \mathrm{~Hz}, 1 \mathrm{H}), 4.86(\mathrm{~d}, J=$ $12.5 \mathrm{~Hz}, 1 \mathrm{H}), 4.80(\mathrm{~d}, J=6.9 \mathrm{~Hz}, 1 \mathrm{H}), 4.73-4.69(\mathrm{~m}, 2 \mathrm{H}), 3.78(\mathrm{~d}, J=9.8 \mathrm{~Hz}, 1 \mathrm{H}), 3.73(\mathrm{~d}, J=$ $9.9 \mathrm{~Hz}, 1 \mathrm{H}), 2.65(\mathrm{~s}, 1 \mathrm{H}), 1.99-1.90(\mathrm{~m}, 1 \mathrm{H}), 1.87-1.74(\mathrm{~m}, 7 \mathrm{H}), 1.68-1.61(\mathrm{~m}, 5 \mathrm{H}), 1.55(\mathrm{~s}$, 1H), $1.26(\mathrm{~s}, 3 \mathrm{H}), 0.90(\mathrm{~s}, 9 \mathrm{H}), 0.84(\mathrm{~s}, 3 \mathrm{H}), 0.061(\mathrm{~s}, 3 \mathrm{H}), 0.055(\mathrm{~s}, 3 \mathrm{H})$.

${ }^{13} \mathbf{C}\left\{{ }^{1} \mathbf{H}\right\}$ NMR $\left(151 \mathrm{MHz}, \mathrm{CDCl}_{3}\right) \delta 155.2,147.7,138.4,137.9,135.9,134.1,131.0,129.0$, $128.6,128.5,127.9,127.9,126.7,125.9,125.3,123.4,122.4,93.8,92.1,78.5,69.7,67.4,64.2$, $60.0,54.1,50.8,50.0,46.8,42.0,26.0,24.6,23.1,22.0,21.5,18.4,11.8,-5.37,-5.43$.

HRMS (ESI/TOF) m/z: [M+Na $]^{+}$Calcd for $\mathrm{C}_{42} \mathrm{H}_{57} \mathrm{NO}_{6} \mathrm{SiNa} 722.3848$; Found 722.3863 . $[\boldsymbol{\alpha}]_{\mathbf{D}}{ }^{\mathbf{2 0 . 9}}=-58.1\left(\mathrm{c} 1.36 \mathrm{CDCl}_{3}\right)$.

Carbamate (+)-27

$\mathbf{R}_{\boldsymbol{f}}=0.48\left(10 \% \mathrm{Et}_{2} \mathrm{O}\right.$ in $\left.\mathrm{CH}_{2} \mathrm{Cl}_{2}\right)$, visualized by $\mathrm{UV}$

${ }^{1} \mathbf{H}$ NMR $\left(600 \mathrm{MHz}, \mathrm{CDCl}_{3}\right){ }^{12} \delta 8.10(\mathrm{~d}, J=8.3 \mathrm{~Hz}, 1 \mathrm{H}), 7.86(\mathrm{~d}, J=8.0 \mathrm{~Hz}, 1 \mathrm{H}), 7.78(\mathrm{~d}, J=$ $7.9 \mathrm{~Hz}, 1 \mathrm{H}), 7.54(\mathrm{t}, J=7.2 \mathrm{~Hz}, 1 \mathrm{H}), 7.49(\mathrm{~m}, 2 \mathrm{H}), 7.46-7.43(\mathrm{~m}, 1 \mathrm{H}), 7.38-7.27(\mathrm{~m}, 5 \mathrm{H})$, 5.70-5.62 (m, 1H), $5.01(\mathrm{~d}, J=7.8 \mathrm{~Hz}, 1 \mathrm{H}), 4.94(\mathrm{~d}, J=12.3 \mathrm{~Hz}, 1 \mathrm{H}), 4.83(\mathrm{~d}, J=12.4 \mathrm{~Hz}, 1 \mathrm{H})$, $4.80(\mathrm{~d}, J=6.7 \mathrm{~Hz}, 1 \mathrm{H}), 4.73-4.68(\mathrm{~m}, 2 \mathrm{H}), 4.55(\mathrm{~d}, J=11.8 \mathrm{~Hz}, 1 \mathrm{H}), 3.77(\mathrm{~d}, J=9.9 \mathrm{~Hz}, 1 \mathrm{H})$, $3.73(\mathrm{~d}, J=9.9 \mathrm{~Hz}, 1 \mathrm{H}), 2.68(\mathrm{~s}, 1 \mathrm{H}), 2.06-1.89(\mathrm{~m}, 1 \mathrm{H}), 1.88-1.73(\mathrm{~m}, 6 \mathrm{H}), 1.70-1.52(\mathrm{~m}, 6 \mathrm{H})$, $1.22(\mathrm{~s}, 3 \mathrm{H}), 0.90(\mathrm{~s}, 9 \mathrm{H}), 0.85(\mathrm{~s}, 3 \mathrm{H}), 0.06(\mathrm{~s}, 3 \mathrm{H}), 0.05(\mathrm{~s}, 3 \mathrm{H})$.

${ }^{13} \mathbf{C}\left\{{ }^{1} \mathbf{H}\right\}$ NMR $\left(151 \mathrm{MHz}, \mathrm{CDCl}_{3}\right) \delta 155.4,147.6,138.4,137.9,136.0,134.0,131.0,128.9$, $128.6,128.5,127.9,127.9,126.6,125.9,125.4,123.4,122.5,93.8,92.1,78.5,69.7,67.3,64.1$, $60.1,54.1,50.6,50.0,46.8,42.0,29.8,26.0,24.6,23.1,22.1,21.5,18.3,11.8,-5.4,-5.5$. HRMS (ESI/TOF) m/z: [M+Na $]^{+}$Calcd for $\mathrm{C}_{42} \mathrm{H}_{57} \mathrm{NO}_{6} \mathrm{SiNa} 722.3848$; Found 722.3844 . $[\boldsymbol{\alpha}]_{\mathbf{D}}{ }^{\mathbf{2 0 . 7}}=59.8\left(\mathrm{c} 1.27 \mathrm{CDCl}_{3}\right)$. 


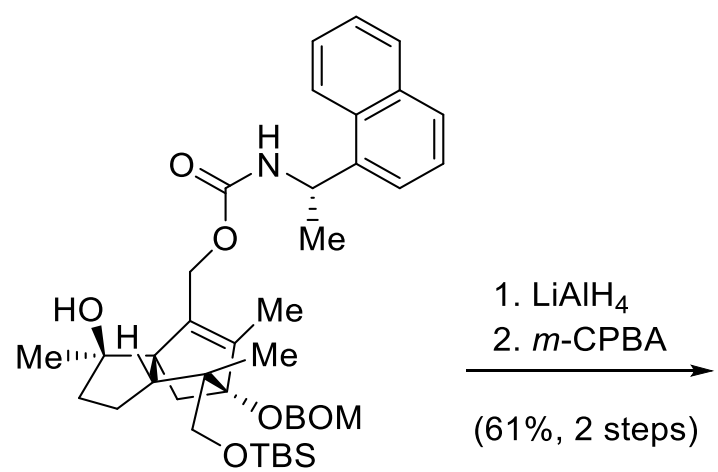

$(-)-26$

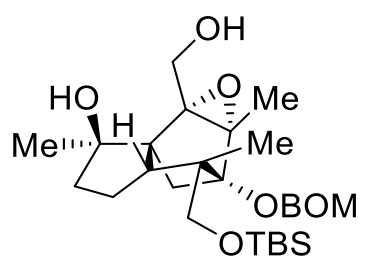

$(-)-16$

Epoxide (-)-16. Carbamate (-)-26 (186 mg, $0.265 \mathrm{mmol})$ was dissolved in $\mathrm{Et}_{2} \mathrm{O}(3 \mathrm{~mL})$. $\mathrm{LiAlH}_{4}$ (33 mg, $0.823 \mathrm{mmol}$ ) was added. The reaction was stirred at ambient temperature. After $4.5 \mathrm{~h}$, TLC analysis showed no more starting material. Saturated Rochelle's salt (aq) (3 mL) was added very slowly until the bubbling had ceased. The mixture was stirred vigorously for $2 \mathrm{~h}$. The mixture was transferred to a separatory funnel and the organic layer was collected. The aqueous layer was washed with diethyl ether $(2 \times 3 \mathrm{~mL})$. The combined organic layers were dried over $\mathrm{MgSO}_{4}$ and concentrated in vacuo to yield the crude alcohol which was used without further purification.

The crude product was dissolved in $\mathrm{CH}_{2} \mathrm{Cl}_{2}$. No attempt was made to exclude air or moisture from the reaction. $m$-CPBA (203 $\mathrm{mg}, 0.823 \mathrm{~mL})$ was added. After $30 \mathrm{~min}$., TLC analysis showed no more starting material. saturated $\mathrm{NaHCO}_{3}(3 \mathrm{~mL})$ and saturated $\mathrm{Na}_{2} \mathrm{~S}_{2} \mathrm{O}_{3}(3 \mathrm{~mL})$ were added, and the mixture was stirred for $1 \mathrm{~h}$. Next, the mixture was transferred to a separatory funnel and the organic layer was collected. The aqueous layer was washed with $\mathrm{CHCl}_{3}(2 \times 75 \mathrm{~mL})$. The combined organic layers were dried over concentrated in vacuo and purified by flash column chromatography on silica (0 - $100 \%$ EtOAc in hexanes gradient), to afford Epoxide (-)-16 (83.6 $\mathrm{mg}, 61 \%$ ) as a foamy white solid. The enantiomeric excess of the product was determined to be 90\%, by HPLC chiral analysis (Chiralcel AD-H with AD guard, $2 \% i$-PrOH in $n$-hexane, 1.0 $\mathrm{mL} / \mathrm{min}, \mathrm{UV}$ detection at $215 \mathrm{~nm}, \mathrm{t}_{\text {major }}=18.6 \mathrm{~min}, \mathrm{t}_{\text {minor }}=22.8 \mathrm{~min}$ ). 
$[\alpha]_{\mathbf{D}^{22.1}}=-56.6\left(\mathrm{c} 1.95 \mathrm{CDCl}_{3}\right)$.

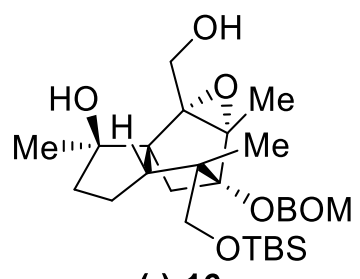

$(-)-16$

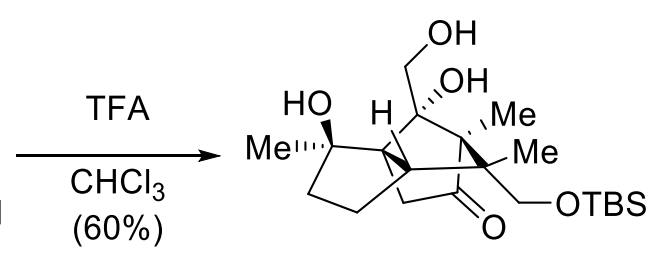

(+)-17

Cyclopentanone triol (+)-17. Epoxide (-)-16 (163 mg, $0.314 \mathrm{mmol})$ was converted to cyclopentanone triol $(+)-17(75.4 \mathrm{mg}, 60 \%)$ following the same procedure for cyclopentanone triol (土)-17.

$[\alpha]_{\mathbf{D}}^{21.5}=4.6\left(\mathrm{c} 4.00 \mathrm{CHCl}_{3}\right)$.

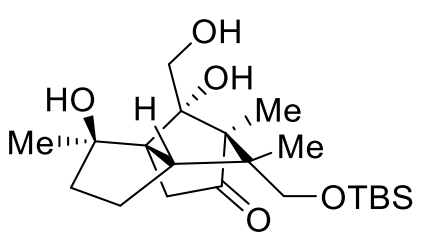

(+)-17

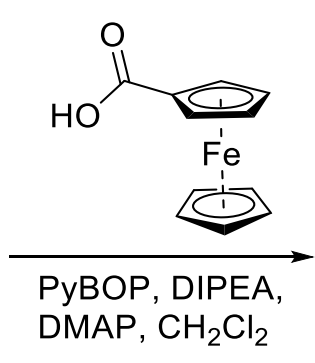

(53\%)

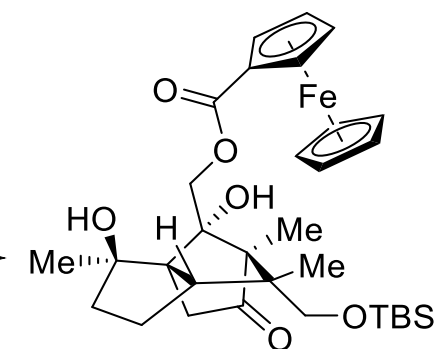

(+)-S9

Ferrocene (+)-S9. Cyclopentanone triol (+)-17 (75.4 mg, 0.189 mmol), ferrocenecarboxylic acid (28) (121 mg, $0.526 \mathrm{mmol})$, DIPEA (0.17 mL, $0.978 \mathrm{mmol})$, DMAP (37 mg, $0.303 \mathrm{mmol})$, and $\mathrm{CH}_{2} \mathrm{Cl}_{2}(1.13 \mathrm{~mL})$ were combined in a flask. PyBOP $(271 \mathrm{mg}, 0.521 \mathrm{mmol})$ was added, and the reaction was stirred overnight. The following morning, saturated $\mathrm{NH}_{4} \mathrm{Cl}(\mathrm{aq})(5 \mathrm{~mL})$ was added. The mixture was transferred to a separatory funnel and the organic layer was collected. The aqueous layer was washed with $\mathrm{CH}_{2} \mathrm{Cl}_{2}(2 \times 5 \mathrm{~mL})$. The combined organic layers were dried over $\mathrm{MgSO}_{4}$, concentrated in vacuo, and purified by flash column chromatography on silica $(0-30 \%$ EtOAc in hexanes gradient), to afford ferrocene $(+)-\mathbf{S 9}(59.1 \mathrm{mg}, 53 \%)$ as a foamy orange solid. $\mathbf{R}_{\boldsymbol{f}}=0.39$ (33\% EtOAc in hexanes), visualized by Hanessian's stain or UV. 
${ }^{1}$ H NMR $\left(500 \mathrm{MHz}, \mathrm{CDCl}_{3}\right) \delta 4.80-4.75(\mathrm{~m}, 4 \mathrm{H}), 4.49-4.45(\mathrm{~m}, 2 \mathrm{H}), 4.25(\mathrm{~s}, 5 \mathrm{H}), 3.25(\mathrm{~d}, J=$ $10.1 \mathrm{~Hz}, 1 \mathrm{H}), 3.18(\mathrm{~d}, J=10.1 \mathrm{~Hz}, 1 \mathrm{H}), 3.04(\mathrm{t}, J=9.8 \mathrm{~Hz}, 1 \mathrm{H}), 2.60(\mathrm{~s}, 1 \mathrm{H}), 2.58(\mathrm{~d}, J=17.5$ $\mathrm{Hz}, 1 \mathrm{H}), 2.49(\mathrm{~s}, 1 \mathrm{H}), 2.17(\mathrm{~d}, J=17.6 \mathrm{~Hz}, 1 \mathrm{H}), 2.11-2.00(\mathrm{~m}, 2 \mathrm{H}), 1.86-1.78(\mathrm{~m}, 1 \mathrm{H}), 1.53$ (ddd, $J=18.8,11.6,6.4 \mathrm{~Hz}, 1 \mathrm{H}), 1.44(\mathrm{~s}, 3 \mathrm{H}), 1.09(\mathrm{~s}, 3 \mathrm{H}), 1.02(\mathrm{~s}, 3 \mathrm{H}), 0.87(\mathrm{~s}, 9 \mathrm{H}), 0.00(\mathrm{~s}$, $3 \mathrm{H}),-0.01(\mathrm{~s}, 3 \mathrm{H})$.

${ }^{13} \mathbf{C}\left\{{ }^{1} \mathbf{H}\right\}$ NMR $\left(126 \mathrm{MHz}, \mathrm{CDCl}_{3}\right) \delta 212.7,172.3,83.2,75.9,72.2,71.7,70.2,70.1,69.6,66.3$, $63.9,63.5,51.6,43.4,39.6,39.3,25.9,25.0,23.4,19.3,18.2,7.1,-5.60,-5.64$.

HRMS (ESI/TOF) m/z: [M+Na] $]^{+}$Calcd for $\mathrm{C}_{32} \mathrm{H}_{46} \mathrm{FeO}_{6} \mathrm{SiNa}_{633.2306}$; Found 633.2330. $[\alpha]_{\mathbf{D}}{ }^{21.7}=3.5\left(\mathrm{c} 1.41 \mathrm{CHCl}_{3}\right)$.

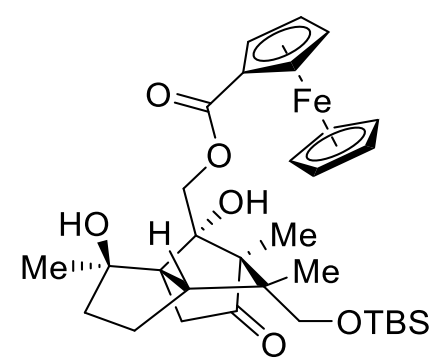

(+)-S9

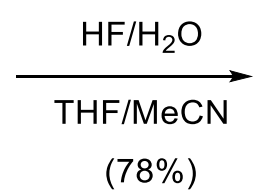

$(78 \%)$

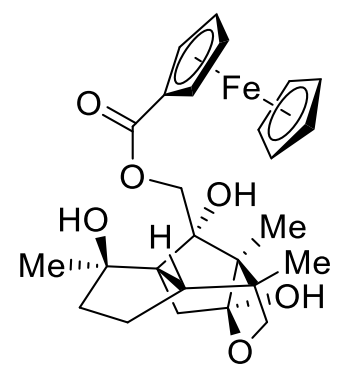

(+)-29

Lactol (+)-29. To ferrocene (+)-S9 $(59.1 \mathrm{mg}, 0.0969 \mathrm{mmol})$ was added THF $(0.4 \mathrm{~mL}), \mathrm{MeCN}$ (0.4 mL), and 49\% HF (aq) $(0.2 \mathrm{~mL})$. The solution was stirred overnight. The following morning silica gel $(0.2 \mathrm{~g})$ was added, and the mixture was stirred for $1 \mathrm{~h}$. The solvent was removed in vacuo, and the impregnated silica was purified by flash column chromatography on silica $(0-100$ \% EtOAc in hexanes gradient), to afford lactol (+)-29 (37.3 mg, 78\%) as an orange solid. Crystals were grown for x-ray diffraction by liquid/liquid diffusion using $\mathrm{CHCl}_{3}$ (solvent) and pentanes (antisolvent). A .cif file is included separately, and the structure has been deposited in the CCDC database: Deposition Number 1945984.

$\mathbf{R}_{\boldsymbol{f}}=0.19$ (50\% EtOAc in hexanes), visualized by Hanessian's stain or UV. 
${ }^{1} \mathbf{H}$ NMR $\left(500 \mathrm{MHz}, \mathrm{CDCl}_{3}\right) \delta 4.79(\mathrm{~s}, 2 \mathrm{H}), 4.72(\mathrm{~d}, J=11.8 \mathrm{~Hz}, 1 \mathrm{H}), 4.47(\mathrm{~d}, J=11.0 \mathrm{~Hz}, 1 \mathrm{H})$, $4.45(\mathrm{~m}, 2 \mathrm{H}), 4.26(\mathrm{~s}, 5 \mathrm{H}), 3.72(\mathrm{~d}, J=8.7 \mathrm{~Hz}, 1 \mathrm{H}), 3.53(\mathrm{~d}, J=8.7 \mathrm{~Hz}, 1 \mathrm{H}), 3.18-3.03$ (br s, 1H), $2.96(\mathrm{t}, J=9.6 \mathrm{~Hz}, 1 \mathrm{H}), 2.80($ br s, $1 \mathrm{H}), 2.75($ br s, $1 \mathrm{H}), 2.36(\mathrm{dd}, J=13.2,1.8 \mathrm{~Hz}, 1 \mathrm{H})$, 2.11-1.99 (m, 2H), $1.87(\mathrm{~d}, J=13.2 \mathrm{~Hz}, 1 \mathrm{H}), 1.73-1.65(\mathrm{~m}, 1 \mathrm{H}), 1.54(\mathrm{~m}, 1 \mathrm{H}), 1.42(\mathrm{~s}, 3 \mathrm{H})$, $1.04(\mathrm{~s}, 3 \mathrm{H}), 0.96(\mathrm{~s}, 3 \mathrm{H})$.

${ }^{13} \mathbf{C}\left\{{ }^{1} \mathbf{H}\right\}$ NMR $\left(126 \mathrm{MHz}, \mathrm{CDCl}_{3}\right) \delta 171.8,111.5,83.0,75.8,72.0,70.2,70.2,70.1,70.1,69.8$, $63.8,63.2,62.9,53.1,43.4,43.3,25.1,21.8,18.7,6.2$.

HRMS (ESI/TOF) m/z: [M+Na $]^{+}$Calcd for $\mathrm{C}_{26} \mathrm{H}_{32} \mathrm{FeO}_{6} \mathrm{Na}$ 519.1442; Found 519.1461.

Melting Point: $155-160{ }^{\circ} \mathrm{C}$

$[\alpha]_{\mathbf{D}}{ }^{21.8}=64.5\left(\mathrm{c} 1.96 \mathrm{CHCl}_{3}\right)$.

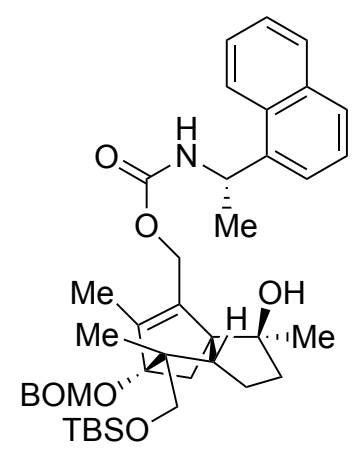

$(+)-27$

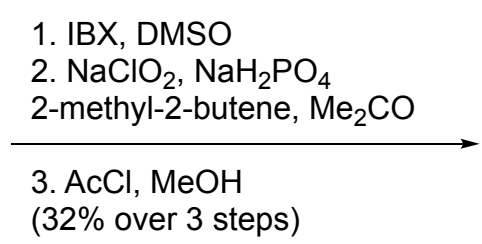

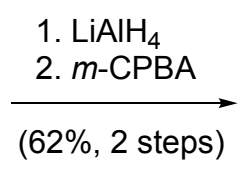

(62\%, 2 steps) eps)

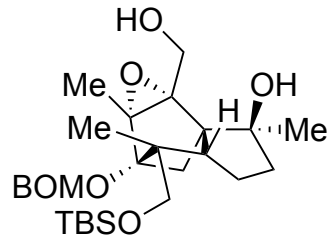

$(+)-16$

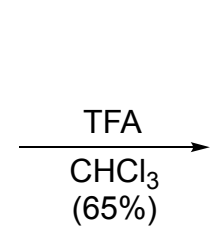

$(65 \%)$

TBSO

$(-)-17$

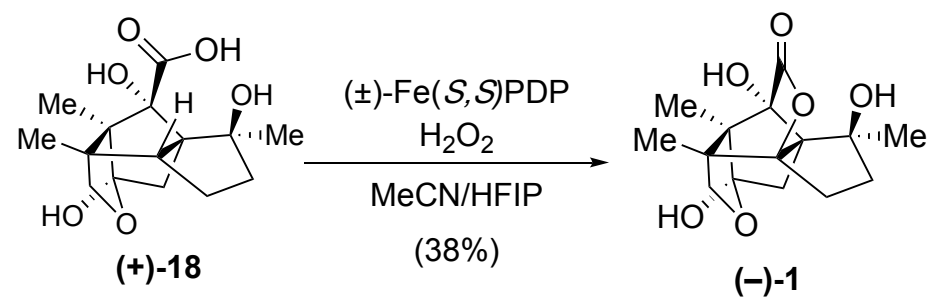

Epoxide (+)-16. Carbamate (+)-27 (192 mg, $0.274 \mathrm{mmol})$ was converted to epoxide $(+)-16(87.7$ $\mathrm{mg}, 62 \%$ ) following the same procedure previously detailed for epoxide (-)-16. The enantiomeric excess of the product was determined to be $91 \%$, by HPLC chiral analysis (Chiralcel AD-H with 
AD guard, $2 \% i-\mathrm{PrOH}$ in $n$-hexane, $1.0 \mathrm{~mL} / \mathrm{min}$, $\mathrm{UV}$ detection at $215 \mathrm{~nm}, \mathrm{t}_{\text {minor }}=18.9 \mathrm{~min}$, $\mathrm{t}_{\text {major }}$ $=22.2 \mathrm{~min})$.

$[\boldsymbol{\alpha}]_{\mathbf{D}}{ }^{21.2}=59.9\left(\mathrm{c} 1.00 \mathrm{CDCl}_{3}\right)$.

Cyclopentanone triol (-)-17. Epoxide (+)-16 (85 $\mathrm{mg}, 0.16 \mathrm{mmol})$ was converted to cyclopentanone triol (-)-17 (42.5 mg, 65\%) following the same procedure for cyclopentanone triol (土)-17.

$[\boldsymbol{\alpha}]_{\mathbf{D}}^{\mathbf{2 1 . 8}}=-10.1\left(\mathrm{c} 0.91 \mathrm{CHCl}_{3}\right)$.

Carboxylic acid (+)-18. Cyclopentanone triol (-)-17 (42.0 mg, $0.105 \mathrm{mmol})$ was converted to carboxylic acid (+)-18 (10.0 mg, 32\% over 3 steps) following the same procedure for carboxylic acid ( \pm )-18.

$[\boldsymbol{\alpha}]_{\mathbf{D}} \mathbf{2 2 . 0}^{2}=3.7(\mathrm{c} 1.00 \mathrm{MeOH})$.

(-)-Illisimonin A. Carboxylic acid (+)-18 (10.0 mg, $0.335 \mathrm{mmol})$ was converted to (-)-illisimonin A (3.8 $\mathrm{mg}, 38 \%)$ following the same procedure for $( \pm)$-illisimonin A, using an equal mixture of $\operatorname{Fe}(R, R) \mathrm{PDP}$ and $\operatorname{Fe}(S, S) \mathrm{PDP}$.

$[\boldsymbol{\alpha}]_{\mathbf{D}}{ }^{\mathbf{2 2 . 0}}=-12.4(\mathrm{c} 0.35 \mathrm{MeOH})$.

$[\alpha]_{577^{22.1}}=-14.8(\mathrm{c} 0.35 \mathrm{MeOH})$.

$[\alpha]_{546}^{22.1}=-17.2($ c $0.35 \mathrm{MeOH})$.

$[\boldsymbol{\alpha}]_{\mathbf{4 3 5}}{ }^{\mathbf{2 2 . 1}}=-35.1(\mathrm{c} 0.35 \mathrm{MeOH})$.

$[\alpha]_{405}^{22.1}=-44.0($ c $0.35 \mathrm{MeOH})$. 


\section{Illismonin A Tabulated NMR data}

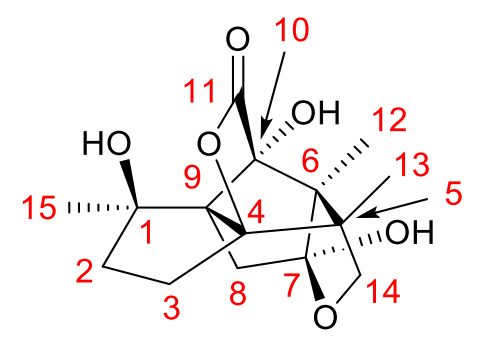

Illisimonin A spectral comparison

\begin{tabular}{|c|c|c|}
\hline Position & $\begin{array}{c}\text { Isolation } \\
{ }^{1} \mathrm{H} \text { NMR }\left(500 \mathrm{MHz}, \mathrm{CD}_{3} \mathrm{OD}\right)\end{array}$ & $\begin{array}{c}\text { Synthetic } \\
{ }^{1} \mathrm{H} \mathrm{NMR}\left(500 \mathrm{MHz}, \mathrm{CD}_{3} \mathrm{OD}\right)\end{array}$ \\
\hline $2 \alpha$ & $2.25(\mathrm{ddd}, J=14.0,10.5,3.5 \mathrm{~Hz}, 1 \mathrm{H})$ & $2.25(\mathrm{ddd}, J=14.0,10.6,3.3 \mathrm{~Hz}, 1 \mathrm{H})$ \\
\hline $2 \beta$ & $2.36(\mathrm{~d}$ app. $\mathrm{t}, J=14.0,8.5 \mathrm{~Hz}, 1 \mathrm{H})$ & $2.37(\mathrm{~d}$ app. $\mathrm{t}, J=14.2,8.7 \mathrm{~Hz}, 1 \mathrm{H})$ \\
\hline $3 \alpha$ & $2.03(\mathrm{ddd}, J=14.5,10.5,8.5 \mathrm{~Hz}, 1 \mathrm{H})$ & $2.02(\mathrm{ddd}, J=14.3,10.3,8.5 \mathrm{~Hz}, 1 \mathrm{H})$ \\
\hline $3 \beta$ & $1.91(\mathrm{ddd}, J=14.5,8.5,3.5 \mathrm{~Hz}, 1 \mathrm{H})$ & $1.91(\mathrm{ddd}, J=14.3,8.9,3.1 \mathrm{~Hz}, 1 \mathrm{H})$ \\
\hline $8 \alpha$ & $1.97(\mathrm{~d}, J=14.5 \mathrm{~Hz}, 1 \mathrm{H})$ & $1.97(\mathrm{~d}, J=14.3 \mathrm{~Hz}, 1 \mathrm{H})$ \\
\hline $8 \beta$ & $2.30(\mathrm{~d}, J=14.5 \mathrm{~Hz}, 1 \mathrm{H})$ & $2.31(\mathrm{~d}, J=14.3 \mathrm{~Hz}, 1 \mathrm{H})$ \\
\hline 12 & $0.91(\mathrm{~s}, 3 \mathrm{H})$ & $0.91(\mathrm{~s}, 3 \mathrm{H})$ \\
\hline 13 & $1.03(\mathrm{~s}, 3 \mathrm{H})$ & $1.03(\mathrm{~s}, 3 \mathrm{H})$ \\
\hline $14 \alpha$ & $3.79(\mathrm{~d}, J=10.0 \mathrm{~Hz}, 1 \mathrm{H})$ & $3.79(\mathrm{~d}, J=10.1 \mathrm{~Hz}, 1 \mathrm{H})$ \\
\hline $14 \beta$ & $3.55(\mathrm{~d}, J=10.0 \mathrm{~Hz}, 1 \mathrm{H})$ & $3.55(\mathrm{~d}, J=10.1 \mathrm{~Hz}, 1 \mathrm{H})$ \\
\hline 15 & $1.27(\mathrm{~s}, 3 \mathrm{H})$ & $1.27(\mathrm{~s}, 3 \mathrm{H})$ \\
\hline
\end{tabular}

\begin{tabular}{|c|c|c|}
\hline Position & Isolation & Synthetic \\
& ${ }^{13} \mathrm{C}\left\{{ }^{1} \mathrm{H}\right\}$ NMR $\left(125 \mathrm{MHz}, \mathrm{CD}_{3} \mathrm{OD}\right)$ & ${ }^{13} \mathrm{C}\left\{{ }^{1} \mathrm{H}\right\}$ \\
\hline 1 & 76.7 & 76.7 \\
\hline 2 & 46.5 & 46.5 \\
\hline 3 & 23.1 & 23.1 \\
\hline 4 & 104.9 & 104.9 \\
\hline 5 & 52.8 & 52.8 \\
\hline 6 & 63.9 & 63.9 \\
\hline 7 & 111.5 & 11.5 \\
\hline 8 & 39.0 & 39.1 \\
\hline 9 & 71.0 & 71.0 \\
\hline 10 & 88.4 & 88.4 \\
\hline 11 & 177.6 & 177.6 \\
\hline 12 & 6.0 & 5.9 \\
\hline 13 & 16.7 & 16.7 \\
\hline 14 & 69.7 & 69.7 \\
\hline 15 & 28.1 & 28.1 \\
\hline
\end{tabular}




\section{(-)-Illisimonin A Circular Dichroism}

Measurements were carried out in a $0.1 \mathrm{~cm}$ cuvette, at room temperature, at $0.79 \mathrm{mg} / \mathrm{mL}$ concentration in $\mathrm{MeOH}$.

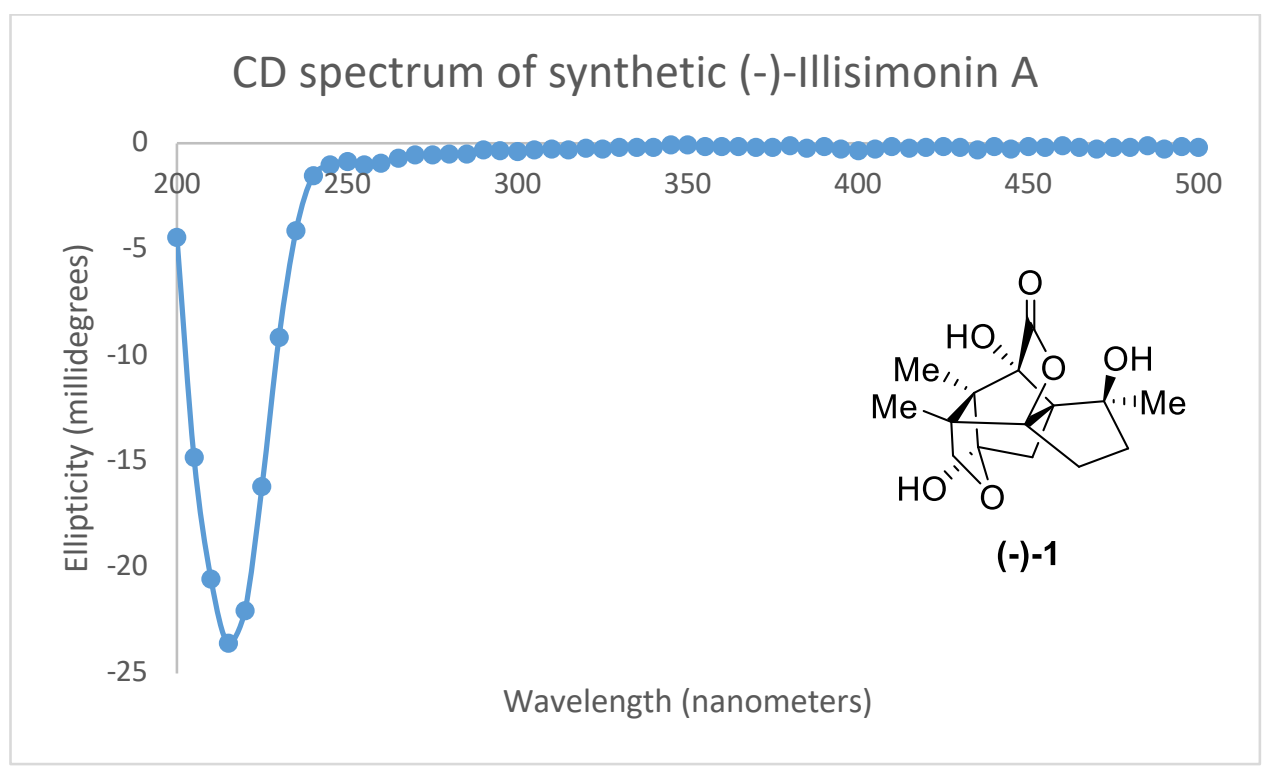

Literature circular dichroism spectrum: ${ }^{13}$

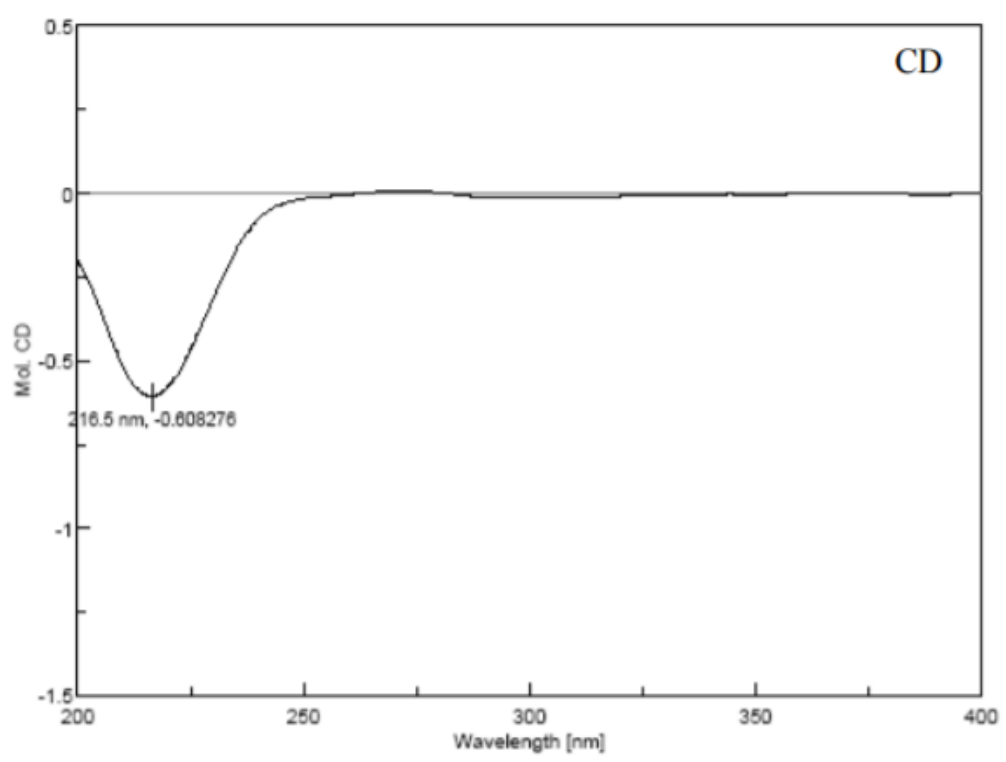




\section{NMR Assignments of compounds 13, 17, 21, and 23}

The relative structure of $\mathbf{1 3}$ was assigned on the basis of ${ }^{1} \mathrm{H},{ }^{13} \mathrm{C}, \mathrm{COSY}$, HSQC, HMBC, and NOESY NMR as well as HRMS analysis. Shown below are the chemical shift assignments:
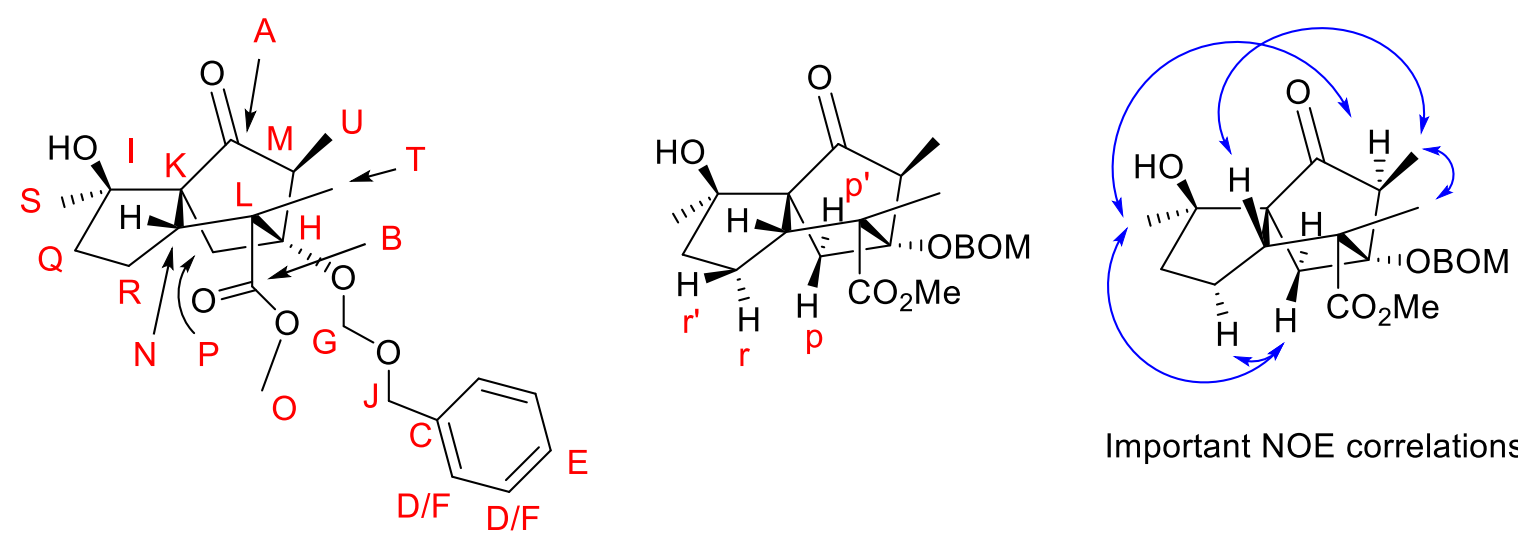

Important NOE correlations

Discussion: The correlations between methine $\mathbf{n}$, methyl $\mathbf{t}$, and methyl $\mathbf{u}$ were strong indicators of the relative stereochemistry about those three stereocenters. The NOE observed between methyl $\mathbf{S}$ and methine $\mathbf{m}$ further confirmed the stereochemistry at carbon $\mathbf{M}$, and established the configuration of carbon $\mathbf{I}$. The observed stereochemistry is consistent with a Diels-Alder templated through silacycle 12. The stereochemistry at carbon $\mathbf{M}$ can be rationalized by protonation from the less hindered alpha face of the resulting silyl-enol ether from the DielsAlder. Tricycle $\mathbf{1 3}$ was ultimately taken on to natural product $\mathbf{1}$, further confirming our structure assignment.

\begin{tabular}{|l|l|l|l|l|}
\hline$\delta(\mathrm{ppm})$ & Splitting & $\mathrm{J}(\mathrm{Hz})$ & Integration & Assignment \\
\hline $7.38-7.28$ & $\mathrm{~m}$ & & $5 \mathrm{H}$ & $\mathrm{d}-\mathrm{f}$ \\
\hline 5.06 & $\mathrm{~d}$ & 7.7 & $1 \mathrm{H}$ & $\mathrm{g}$ \\
\hline 4.84 & $\mathrm{~d}$ & 7.7 & $1 \mathrm{H}$ & $\mathrm{g}$ \\
\hline 4.68 & $\mathrm{~d}$ & 11.7 & $1 \mathrm{H}$ & $\mathrm{j}$ \\
\hline 4.62 & $\mathrm{~d}$ & 11.7 & $1 \mathrm{H}$ & $\mathrm{j}$ \\
\hline 4.27 & $\mathrm{~s}$ & & $1 \mathrm{H}$ & $\mathrm{O}-\mathrm{H}$ \\
\hline 3.71 & $\mathrm{~s}$ & & $3 \mathrm{H}$ & $\mathrm{o}$ \\
\hline 3.04 & $\mathrm{~d}$ & 10.0 & $1 \mathrm{H}$ & $\mathrm{p}$ \\
\hline 2.96 & $\mathrm{q}$ & 7.1 & $1 \mathrm{H}$ & $\mathrm{m}$ \\
\hline 2.34 & $\mathrm{ddd}$ & $10.8,6.0,2.1$ & $1 \mathrm{H}$ & $\mathrm{n}$ \\
\hline
\end{tabular}




\begin{tabular}{|l|l|l|l|l|}
\hline $2.15-2.03$ & $\mathrm{~m}$ & & $1 \mathrm{H}$ & $\mathrm{r}$ \\
\hline 1.95 & $\mathrm{dd}$ & $9.9,2.2$ & $1 \mathrm{H}$ & $\mathrm{p}$ \\
\hline $1.87-1.79$ & $\mathrm{~m}$ & & $2 \mathrm{H}$ & $\mathrm{q}, \mathrm{q}^{\prime}$ \\
\hline $1.53-1.43$ & $\mathrm{~m}$ & & $1 \mathrm{H}$ & $\mathrm{r}$ \\
\hline 1.43 & $\mathrm{~s}$ & & $3 \mathrm{H}$ & $\mathrm{t}$ \\
\hline 1.30 & $\mathrm{~d}$ & 7.1 & $3 \mathrm{H}$ & $\mathrm{u}$ \\
\hline 1.30 & $\mathrm{~s}$ & & $3 \mathrm{H}$ & $\mathrm{s}$ \\
\hline
\end{tabular}

\begin{tabular}{|l|l|}
\hline$\delta(\mathrm{ppm})$ & Assignment \\
\hline 217.8 & $\mathrm{~A}$ \\
\hline 174.1 & $\mathrm{~B}$ \\
\hline 137.5 & $\mathrm{C}$ \\
\hline 128.7 & $\mathrm{D}$ \\
\hline 128.07 & $\mathrm{E}$ \\
\hline 128.05 & $\mathrm{~F}$ \\
\hline 92.4 & $\mathrm{G}$ \\
\hline 89.0 & $\mathrm{H}$ \\
\hline 79.7 & $\mathrm{I}$ \\
\hline 70.2 & $\mathrm{~J}$ \\
\hline 68.2 & $\mathrm{~K}$ \\
\hline 58.2 & $\mathrm{~L}$ \\
\hline 57.1 & $\mathrm{M}$ \\
\hline 56.1 & $\mathrm{~N}$ \\
\hline 51.9 & $\mathrm{O}$ \\
\hline 40.5 & $\mathrm{P}$ \\
\hline 40.3 & $\mathrm{Q}$ \\
\hline 25.1 & $\mathrm{R}$ \\
\hline 24.5 & $\mathrm{~S}$ \\
\hline 21.8 & $\mathrm{~T}$ \\
\hline 10.2 & $\mathrm{U}$ \\
\hline & \\
\hline
\end{tabular}

The relative structure of 21 was assigned on the basis of ${ }^{1} \mathrm{H},{ }^{13} \mathrm{C}, \mathrm{COSY}, \mathrm{HSQC}, \mathrm{HMBC}$, and NOESY NMR as well as HRMS analysis. Shown below are the chemical shift assignments: 

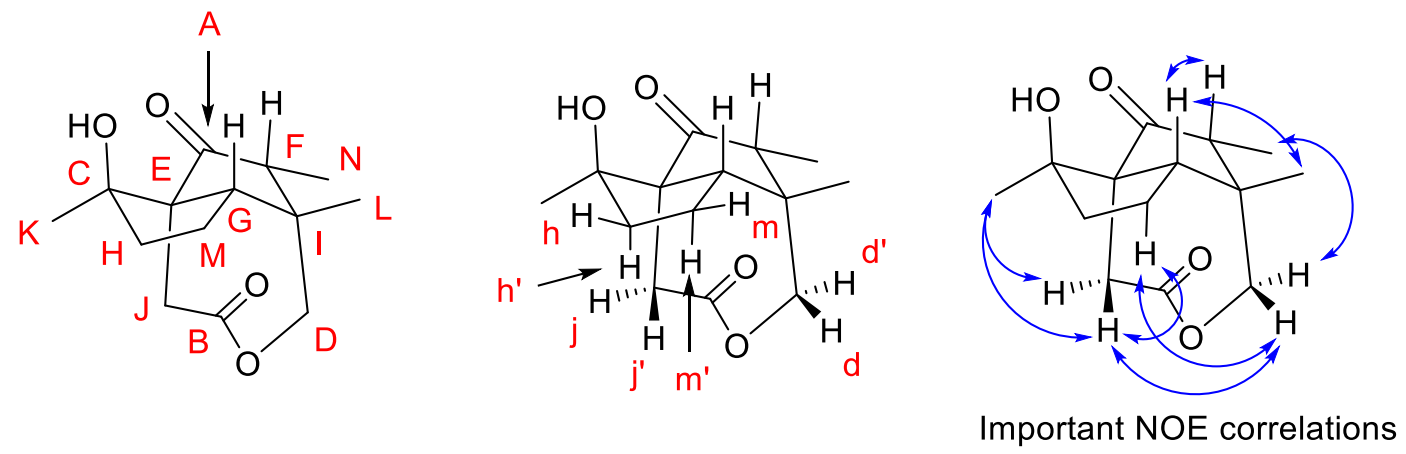

Discussion: The NOE between methines $\mathbf{f}$ and $\mathbf{g}$ were helpful in establishing the stereochemistry at carbon $\mathbf{F}$. The rest of the structure was consistent with the proposed semi-pinacol/retroClaisen sequence from intermediate 19, whose structure can be traced back to tricycle $\mathbf{1 3}$ through standard transformations.

\begin{tabular}{|l|l|l|l|l|}
\hline$\delta(\mathrm{ppm})$ & Splitting & $\mathrm{J}(\mathrm{Hz})$ & Integration & Assignment \\
\hline 4.42 & $\mathrm{~d}$ & 13.4 & $1 \mathrm{H}$ & $\mathrm{d}$ \\
\hline 3.98 & $\mathrm{dd}$ & $13.4,1.3$ & $1 \mathrm{H}$ & $\mathrm{d}$ \\
\hline 3.10 & $\mathrm{dd}$ & $12.9,6.9$ & $1 \mathrm{H}$ & $\mathrm{g}$ \\
\hline 2.76 & $\mathrm{dd}$ & $15.0,0.9$ & $1 \mathrm{H}$ & $\mathrm{j}$ \\
\hline 2.63 & $\mathrm{~d}$ & 14.9 & $1 \mathrm{H}$ & $\mathrm{j}$ \\
\hline 2.44 & $\mathrm{q}$ & 7.3 & $1 \mathrm{H}$ & $\mathrm{f}$ \\
\hline 2.21 & $\mathrm{ddd}$ & $15.3,9.1,6.5$ & $1 \mathrm{H}$ & $\mathrm{h}$ \\
\hline $2.08-2.02$ & $\mathrm{~m}$ & & $1 \mathrm{H}$ & $\mathrm{h}$ \\
\hline $1.79-1.73$ & $\mathrm{~m}$ & & $1 \mathrm{H}$ & $\mathrm{m}$ \\
\hline 1.66 & $\mathrm{app} . \mathrm{tdd}$ & $12.5,10.9,6.4$ & $1 \mathrm{H}$ & $\mathrm{m}$ \\
\hline 1.46 & $\mathrm{~s}$ & & $3 \mathrm{H}$ & $\mathrm{k}$ \\
\hline 1.22 & $\mathrm{~s}$ & & $3 \mathrm{H}$ & $\mathrm{n}$ \\
\hline 1.14 & $\mathrm{~s}$ & & $3 \mathrm{H}$ & 1 \\
\hline
\end{tabular}

\begin{tabular}{|l|l|}
\hline$\delta(\mathrm{ppm})$ & Assignment \\
\hline 213.6 & $\mathrm{~A}$ \\
\hline 171.0 & $\mathrm{~B}$ \\
\hline 77.0 & $\mathrm{C}$ \\
\hline 69.0 & $\mathrm{D}$ \\
\hline 64.2 & $\mathrm{E}$ \\
\hline 62.1 & $\mathrm{~F}$ \\
\hline 54.7 & $\mathrm{G}$ \\
\hline 42.6 & $\mathrm{H}$ \\
\hline 40.6 & $\mathrm{I}$ \\
\hline 35.1 & $\mathrm{~J}$ \\
\hline
\end{tabular}




\begin{tabular}{|l|l|}
\hline 24.7 & $\mathrm{~K}$ \\
\hline 23.1 & $\mathrm{~L}$ \\
\hline 16.4 & $\mathrm{M}$ \\
\hline 8.8 & $\mathrm{~N}$ \\
\hline
\end{tabular}

The relative structure of $\mathbf{1 7}$ was assigned on the basis of ${ }^{1} \mathrm{H},{ }^{13} \mathrm{C}, \mathrm{COSY}$, HSQC, HMBC, and NOESY NMR as well as HRMS analysis. Shown below are the chemical shift assignments:
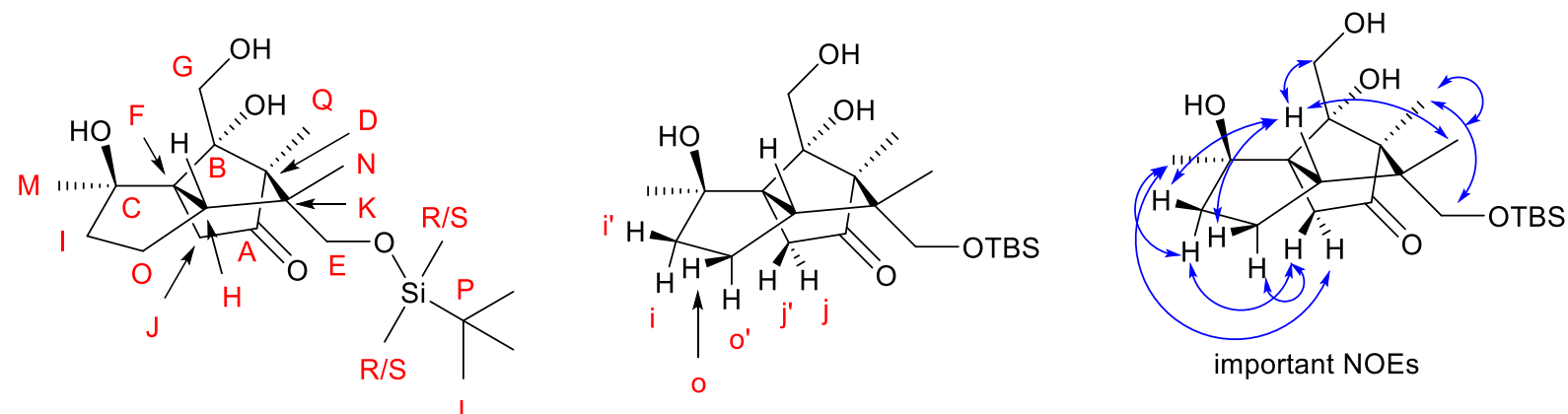

Discussion: The NOE's between methylene protons $\mathbf{g}$, methine $\mathbf{h}$, and methyl $\mathbf{n}$, as well as between methylene g' and methyl q were helpful in establishing the configuration of carbon $\mathbf{B}$.

The appearance of a ketone (A), where there previously had been none was helpful in confirming the desired semipinacol rearrangement had taken place. Finally, intermediate $\mathbf{1 7}$ was taken forward to natural product $\mathbf{1}$, further confirming its relative structure.

\begin{tabular}{|l|l|l|l|l|}
\hline$\delta(\mathrm{ppm})$ & Splitting & $\mathrm{J}(\mathrm{Hz})$ & Integration & Assignment \\
\hline 4.40 & $\mathrm{~d}$ & 11.4 & $1 \mathrm{H}$ & $\mathrm{g}$ \\
\hline 4.19 & br s & & $1 \mathrm{H}$ & $\mathrm{O}-\mathrm{H}$ \\
\hline 3.35 & $\mathrm{~d}$ & 11.7 & $1 \mathrm{H}$ & $\mathrm{g}$ \\
\hline 3.28 & br s & & $1 \mathrm{H}$ & $\mathrm{O}-\mathrm{H}$ \\
\hline 3.22 & $\mathrm{~d}$ & 10.0 & $1 \mathrm{H}$ & $\mathrm{e}$ \\
\hline 3.13 & $\mathrm{~d}$ & 10.1 & $1 \mathrm{H}$ & $\mathrm{e}$ \\
\hline 2.92 & $\mathrm{ddd}$ & $12.0,8.1,1.7$ & $1 \mathrm{H}$ & $\mathrm{h}$ \\
\hline 2.52 & $\mathrm{dd}$ & $17.5,1.9$ & $1 \mathrm{H}$ & $\mathrm{j}$ \\
\hline 2.45 & br s & & $1 \mathrm{H}$ & $\mathrm{O}-\mathrm{H}$ \\
\hline 2.14 & $\mathrm{ddd}$ & $14.8,11.3,3.4$ & $1 \mathrm{H}$ & $\mathrm{i}$ \\
\hline 2.09 & $\mathrm{~d}$ & 17.4 & $1 \mathrm{H}$ & $\mathrm{j}$ \\
\hline 1.96 & $\mathrm{ddd}$ & $14.9,9.1,5.8$ & $1 \mathrm{H}$ & $\mathrm{i}$ \\
\hline 1.86 & $\mathrm{~m}$ & & $1 \mathrm{H}$ & $\mathrm{o}$ \\
\hline 1.53 & $\mathrm{~m}$ & & $1 \mathrm{H}$ & $\mathrm{o}$ \\
\hline 1.36 & $\mathrm{~s}$ & & $3 \mathrm{H}$ & $\mathrm{m}$ \\
\hline
\end{tabular}




\begin{tabular}{|l|l|l|l|l|}
\hline 1.01 & s & & $3 \mathrm{H}$ & $\mathrm{n}$ \\
\hline 0.89 & $\mathrm{~s}$ & & $3 \mathrm{H}$ & $\mathrm{q}$ \\
\hline 0.86 & $\mathrm{~s}$ & & $9 \mathrm{H}$ & $\mathrm{l}$ \\
\hline-0.02 & $\mathrm{~s}$ & & $3 \mathrm{H}$ & $\mathrm{r} / \mathrm{s}$ \\
\hline-0.03 & $\mathrm{~s}$ & & $3 \mathrm{H}$ & $\mathrm{r} / \mathrm{s}$ \\
\hline
\end{tabular}

\begin{tabular}{|l|l|}
\hline$\delta(\mathrm{ppm})$ & Assignment \\
\hline 213.3 & A \\
\hline 84.1 & $\mathrm{~B}$ \\
\hline 76.7 & $\mathrm{C}$ \\
\hline 69.8 & $\mathrm{D}$ \\
\hline 66.0 & $\mathrm{E}$ \\
\hline 63.1 & $\mathrm{~F}$ \\
\hline 60.6 & $\mathrm{G}$ \\
\hline 51.6 & $\mathrm{H}$ \\
\hline 45.3 & $\mathrm{I}$ \\
\hline 39.5 & $\mathrm{~J}$ \\
\hline 39.4 & $\mathrm{~K}$ \\
\hline 25.9 & $\mathrm{~L}$ \\
\hline 25.0 & $\mathrm{M}$ \\
\hline 23.5 & $\mathrm{~N}$ \\
\hline 19.7 & $\mathrm{O}$ \\
\hline 18.2 & $\mathrm{P}$ \\
\hline 6.4 & $\mathrm{Q}$ \\
\hline-5.6 & $\mathrm{R}$ \\
\hline-5.7 & $\mathrm{~S}$ \\
\hline
\end{tabular}

The relative structure of $\mathbf{2 3}$ was assigned on the basis of ${ }^{1} \mathrm{H},{ }^{13} \mathrm{C}, \mathrm{COSY}$, HSQC, HMBC, and NOESY NMR as well as HRMS analysis. Shown below are the chemical shift assignments:
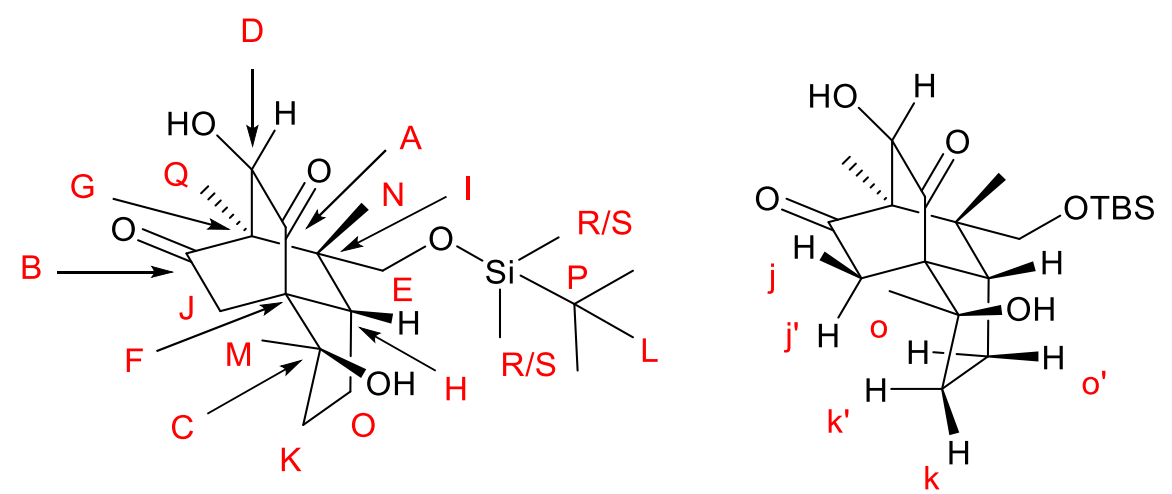

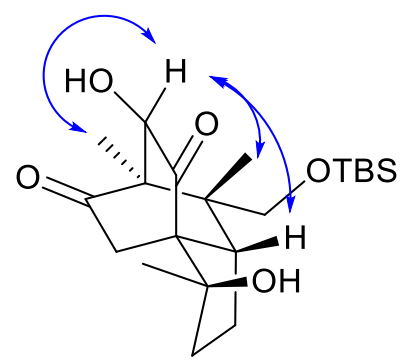

important NOEs 
Discussion: Most important to structure determination were the NOEs between proton $\mathbf{d}$, and methyls $\mathbf{q}$ and $\mathbf{n}$, and methine $\mathbf{h}$. The structure is consistent with an alpha-ketol rearrangement of aldehyde $\mathbf{2 2}$ which can be traced back to primary alcohol 17 (whose structure was earlier established) through simple IBX oxidation.

\begin{tabular}{|l|l|l|l|l|}
\hline$\delta(\mathrm{ppm})$ & Splitting & $\mathrm{J}(\mathrm{Hz})$ & Integration & Assignment \\
\hline 4.08 & $\mathrm{~s}$ & & $1 \mathrm{H}$ & $\mathrm{d}$ \\
\hline 3.50 & $\mathrm{~d}$ & 10.8 & $1 \mathrm{H}$ & $\mathrm{e}$ \\
\hline 3.46 & $\mathrm{~d}$ & 10.8 & $1 \mathrm{H}$ & $\mathrm{e}$ \\
\hline 2.88 & br s & & $1 \mathrm{H}$ & $\mathrm{O}-\mathrm{H}$ \\
\hline 2.77 & app. t & 9.8 & $1 \mathrm{H}$ & $\mathrm{h}$ \\
\hline 2.70 & $\mathrm{~d}$ & 18 & $1 \mathrm{H}$ & $\mathrm{j}$ \\
\hline $2.17-2.11$ & $\mathrm{~m}$ & & $1 \mathrm{H}$ & $\mathrm{o}$ \\
\hline 2.12 & $\mathrm{dd}$ & $17.8,1.9$ & $1 \mathrm{H}$ & $\mathrm{j}$ \\
\hline $2.05-1.96$ & $\mathrm{~m}$ & & $1 \mathrm{H}$ & $\mathrm{o}$ \\
\hline 1.92 & $\mathrm{ddd}$ & $15.3,9.7,5.8$ & $1 \mathrm{H}$ & $\mathrm{k}$ \\
\hline $1.84-1.76$ & $\mathrm{~m}$ & & $1 \mathrm{H}$ & $\mathrm{k}$ \\
\hline 1.37 & $\mathrm{~s}$ & & $3 \mathrm{H}$ & $\mathrm{m}$ \\
\hline 1.12 & $\mathrm{~s}$ & & $3 \mathrm{H}$ & $\mathrm{q}$ \\
\hline 1.00 & $\mathrm{~s}$ & & $3 \mathrm{H}$ & $\mathrm{n}$ \\
\hline 0.87 & $\mathrm{~s}$ & & $9 \mathrm{H}$ & $\mathrm{l}$ \\
\hline 0.05 & $\mathrm{~s}$ & & $3 \mathrm{H}$ & $\mathrm{r} / \mathrm{s}$ \\
\hline 0.03 & $\mathrm{~s}$ & & $3 \mathrm{H}$ & $\mathrm{r} / \mathrm{s}$ \\
\hline
\end{tabular}

\begin{tabular}{|l|l|}
\hline$\delta(\mathrm{ppm})$ & Assignment \\
\hline 214.4 & $\mathrm{~A}$ \\
\hline 206.8 & $\mathrm{~B}$ \\
\hline 79.2 & $\mathrm{C}$ \\
\hline 74.8 & $\mathrm{D}$ \\
\hline 66.6 & $\mathrm{E}$ \\
\hline 60.0 & $\mathrm{~F}$ \\
\hline 57.2 & $\mathrm{G}$ \\
\hline 46.2 & $\mathrm{H}$ \\
\hline 42.2 & $\mathrm{I}$ \\
\hline 38.8 & $\mathrm{~J}$ \\
\hline 38.2 & $\mathrm{~K}$ \\
\hline 26.0 & $\mathrm{~L}$ \\
\hline 24.4 & $\mathrm{M}$ \\
\hline 22.3 & $\mathrm{~N}$ \\
\hline 20.6 & $\mathrm{O}$ \\
\hline 18.5 & $\mathrm{P}$ \\
\hline
\end{tabular}




\begin{tabular}{|l|l|}
\hline 9.0 & $\mathrm{Q}$ \\
\hline-5.9 & $\mathrm{R}$ \\
\hline-6.0 & $\mathrm{~S}$ \\
\hline
\end{tabular}




\section{References}

1 Khadem, El, H.; Hanessian, S. Ammonium Molybdate as Spraying Agent for Paper Chromatograms of Reducing Sugars. Anal. Chem. 1958, 30, 1965-1965.

${ }^{2}$ Chen, M. S.; Science, M. W. A Predictably Selective Aliphatic C-H Oxidation Reaction for Complex Molecule Synthesis. Science 2007, 318, 783-787.

3 (a) Kende, A. S.; Fludzinski, P.; Hill, J. H.; Swenson, W.; Clardy, J. Chloroacetylenes as Michael Acceptors. 3. Mechanism and Synthetic Utility of Enolate Reactions with Halogenated Olefins and Chloroacetylenes. J. Am. Chem. Soc. 1984, 106, 3551-3562. (b) Banwell, M. G.; Jury, J. C. Stereoselective Syntheses of the Methyl Esters of $(E)$ - and (Z)-2-Methyl-6-Oxohept-2-eoic Acid. Org. Prep. Proced. Int. 2004, 36, 87-91.

${ }^{4}$ On smaller scale, the reaction could be run using a magnetic stirrer. On larger scale, the viscous reaction mixture could not be efficiently stirred without overhead stirring.

${ }^{5}$ BOM cleavage was a major side reaction in the absence of extra pyridine.

${ }^{6}$ Renata, H.; Zhou, Q.; Baran, P. S. Strategic Redox Relay Enables a Scalable Synthesis of Ouabagenin, A Bioactive Cardenolide. Science 2013, 339, 59-63.

7 The chemical shifts and splitting for this compound varied at the hydroxyl protons and hydroxyl-methylene protons, depending on the sample, and presumably its concentration and water content. A different sample was used for the 2-D analysis shown in the structural assignments section.

${ }^{8}$ The buffer was prepared by making a $100 \mathrm{~mL}$ aqueous solution of $\mathrm{KHSO}_{4}(3.2 \mathrm{~g}, 24 \mathrm{mmol})$ and $\mathrm{NaH}_{2} \mathrm{PO}_{4}(10$ g. $83 \mathrm{mmol})$ as described in: Xu, C.; Han, A.; Reisman, S. E. An Oxidative Dearomatization Approach to Prepare the Pentacyclic Core of Ryanodol. Org. Lett. 2018, 20, 3793-3796.

${ }^{9}$ This peak corresponds to two overlapping diastereomeric methyls from the TBS moiety.

${ }^{10}$ Vermeulen, N. A.; Chen, M. S.; White, C. The Fe(PDP)-catalyzed aliphatic C-H oxidation: a slow addition protocol. Tetrahedron 2009, 65, 3078-3084.

${ }^{11}$ Chen, K.-F.; Tai, W.-T.; Hsu, C.-Y.; Huang, J.-W.; Liu, C.-Y.; Chen, P.-J.; Kim, I.; Shiau, C.W. Eur. J. Med. Chem. 2012, 55, 220-227.

12 The spectrum is a mixture of rotamers. For clarity, we tabulated only the major rotamer.

${ }^{13}$ Ma, S.-G.; Li, M.; Lin, M.-B.; Li, L.; Liu, Y.-B.; Qu, J.; Li, Y.; Wang, X.-J.; Wang, R.-B.; Xu, S.; Hou, Q.; Yu, S.-S. Illisimonin A, a Caged Sesquiterpenoid with a Tricyclo[5.2.1.0 1,6]decane Skeleton from the Fruits of Illicium simonsii. Org. Lett. 2017, 19, 6160-6163. 
The minimum energy transitions states for exo 10 and for endo 10 are reported. Geometries were optimized using $\omega \mathrm{B} 97 \times \mathrm{xd} / 6-31 \mathrm{G}(\mathrm{d})$ and included full frequency analysis. The strain energy calculations for alkanes 8 and 9 were carried out with MP2/6-31G(d) as reported by Dudev and Lim (reference 17). Calculations were performed using Gaussian $16^{1}$ and Spartan $18 .{ }^{2}$ Images were processed with CYLView 1.0. ${ }^{3}$

\section{Calculated endo transition state for 10:}

SCF Done: $E(R w B 97 X D)=-1368.09578512$

Sum of electronic and thermal Free Energies= $\quad-1367.707501$

(Temperature 298.150 Kelvin. Pressure 1.00000 Atm.)

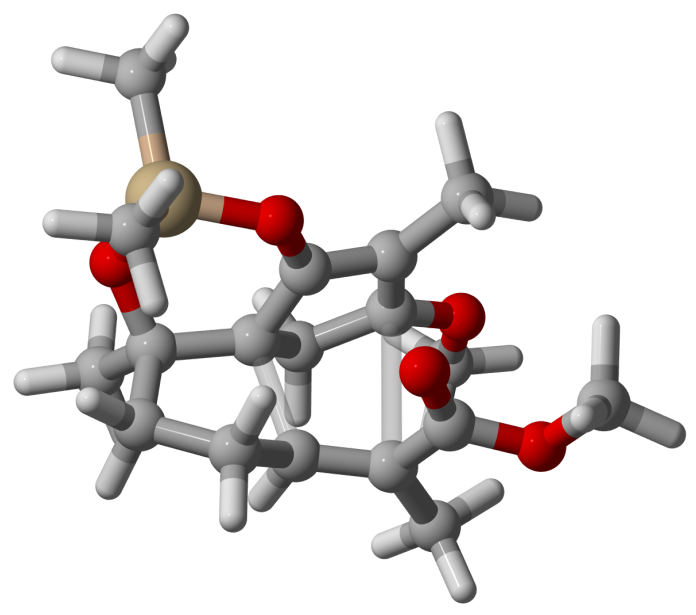

\section{2}

Z_endo_M0001_TS

C $\quad-0 . \overline{4} 86800-0.968100-0.178900$

C $\quad 0.553800 \quad 0.413700 \quad-1.694200$

C $\quad-0.576700 \quad-0.405800-2.294000$

C $\quad-1.786200 \quad 0.530300 \quad-2.260900$

$\begin{array}{llll}\text { C } & -1.816100 & 1.271500 & -0.886500\end{array}$

$\mathrm{H} \quad-2.742300 \quad 0.025800 \quad-2.426000$

H $\quad-1.681500 \quad 1.272100 \quad-3.062400$

C $\quad-0.444700 \quad-0.081700 \quad 0.782900$

C $\quad 0.724500 \quad-0.039300 \quad 1.567400$

C $\quad 1.371800 \quad 1.120700 \quad 1.220000$

$\begin{array}{llll}\text { C } & 0.480800 & 1.999300 & 0.385500\end{array}$

H $\quad 1.009500 \quad 2.587600 \quad-0.366700$

H $\quad-0.045300 \quad 2.696100 \quad 1.053500$

C $\quad \begin{array}{llll}1.853900 & -0.013200 & -1.346500\end{array}$

C $\quad 1.178200 \quad-1.108300 \quad 2.504600$

H $\quad 2.184400 \quad-0.897100 \quad 2.874700$

H $\quad 1.179900 \quad-2.070000 \quad 1.982700$

H $\quad 0.502700 \quad-1.191200 \quad 3.363200$

C $\quad-2.041900 \quad 2.766600 \quad-1.072400$

H $\quad-2.140500 \quad 3.264500 \quad-0.103300$ 


$\begin{array}{rrrr}\mathrm{H} & -2.963400 & 2.930200 & -1.639300 \\ \mathrm{H} & -1.210700 & 3.219500 & -1.623300 \\ \mathrm{O} & -2.931100 & 0.808900 & -0.102900 \\ \mathrm{O} & 2.542500 & 1.487200 & 1.747300 \\ \mathrm{C} & 3.017200 & 2.793300 & 1.464000 \\ \mathrm{H} & 3.951000 & 2.897000 & 2.016600 \\ \mathrm{H} & 2.304900 & 3.553300 & 1.806400 \\ \mathrm{H} & 3.211300 & 2.923300 & 0.395400 \\ \mathrm{O} & -1.361800 & -1.044700 & 0.925600 \\ \mathrm{Si} & -2.981800 & -0.697700 & 0.575100 \\ \mathrm{C} & -3.598300 & -2.056100 & -0.539500 \\ \mathrm{H} & -3.599400 & -3.011600 & -0.003100 \\ \mathrm{H} & -2.959800 & -2.172000 & -1.420200 \\ \mathrm{H} & -4.621400 & -1.857200 & -0.877100 \\ \mathrm{C} & -3.952800 & -0.582600 & 2.157400 \\ \mathrm{H} & -3.957200 & -1.542600 & 2.684600 \\ \mathrm{H} & -4.992700 & -0.300700 & 1.959900 \\ \mathrm{H} & -3.517900 & 0.171600 & 2.820400 \\ \mathrm{H} & -0.331600 & -0.698900 & -3.321400 \\ \mathrm{H} & -0.724400 & -1.322100 & -1.718600 \\ \mathrm{H} & 0.585900 & 1.389300 & -2.194000 \\ \mathrm{C} & 3.067400 & 0.849100 & -1.600000 \\ \mathrm{H} & 2.768100 & 1.868200 & -1.876100 \\ \mathrm{H} & 3.684700 & 0.462200 & -2.422100 \\ \mathrm{H} & 3.724100 & 0.905900 & -0.723500 \\ \mathrm{C} & 2.039100 & -1.343400 & -0.837300 \\ \mathrm{O} & 1.152200 & -2.148500 & -0.561600 \\ \mathrm{O} & 3.353800 & -1.652500 & -0.636100 \\ \mathrm{C} & 3.594700 & -2.939200 & -0.095300 \\ \mathrm{H} & 3.121100 & -3.718200 & -0.699200 \\ \mathrm{H} & 3.214200 & -3.015500 & 0.928500 \\ \mathrm{H} & 4.678900 & -3.064500 & -0.096900\end{array}$




\section{Calculated exo transition state for 10:}

SCF Done: $E(R w B 97 X D)=-1368.10708808$

Sum of electronic and thermal Free Energies $=\quad-1367.721113$ (Temperature 298.150 Kelvin. Pressure 1.00000 Atm.)

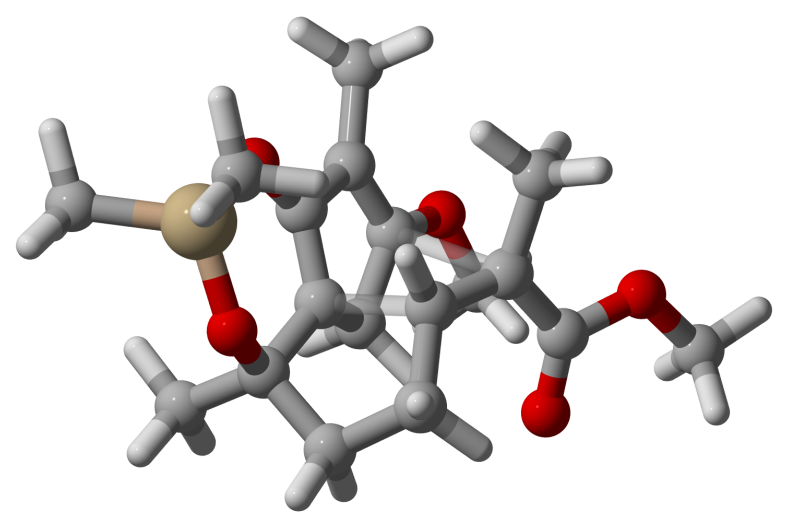

\begin{tabular}{lrrr}
\multicolumn{4}{l}{52} \\
Z_exo_M0001_TS & \\
C & 0.549200 & -0.117500 & 0.701400 \\
C & -0.256600 & -1.023100 & -0.850300 \\
C & -0.044600 & -2.466000 & -0.370700 \\
C & 0.729200 & -2.513500 & 0.955200 \\
C & 1.515500 & -1.212000 & 1.103000 \\
H & 0.506600 & -0.743100 & -1.578100 \\
H & -1.005000 & -2.969200 & -0.260600 \\
H & 0.527300 & -2.982000 & -1.148500 \\
H & 1.405500 & -3.372000 & 1.016900 \\
H & 0.025800 & -2.585100 & 1.792900 \\
C & 0.951900 & 1.107900 & 0.131500 \\
C & -0.087100 & 2.060300 & 0.156300 \\
C & -1.121500 & 1.481300 & 0.861900 \\
C & -0.679700 & 0.209700 & 1.520500 \\
H & -1.429300 & -0.584700 & 1.530600 \\
H & -0.392100 & 0.437100 & 2.557600 \\
C & -1.510700 & -0.454000 & -1.169000 \\
C & -1.608000 & 0.563500 & -2.282200 \\
H & -2.288700 & 1.384200 & -2.033000 \\
H & -1.972700 & 0.119300 & -3.218800 \\
H & -0.621200 & 0.995000 & -2.485900 \\
C & -2.722500 & -0.968700 & -0.591500 \\
O & -3.837800 & -0.491500 & -1.216600 \\
C & -0.100100 & 3.383600 & -0.537800 \\
H & -0.626100 & 4.134200 & 0.059500 \\
H & -0.609300 & 3.314300 & -1.506100 \\
H & 0.920800 & 3.729500 & -0.720000 \\
C & 2.106300 & -1.042200 & 2.501900
\end{tabular}




$\begin{array}{rrrr}\mathrm{H} & 2.611900 & -0.075500 & 2.599600 \\ \mathrm{H} & 2.834200 & -1.837000 & 2.692700 \\ \mathrm{H} & 1.325600 & -1.097200 & 3.266700 \\ \mathrm{O} & 2.590300 & -1.246400 & 0.148800 \\ \mathrm{O} & -2.271300 & 2.119300 & 1.079100 \\ \mathrm{C} & -3.246200 & 1.457700 & 1.874700 \\ \mathrm{H} & -4.132000 & 2.092100 & 1.849300 \\ \mathrm{H} & -2.897300 & 1.357000 & 2.909200 \\ \mathrm{H} & -3.480800 & 0.469100 & 1.472800 \\ \mathrm{O} & -2.833000 & -1.737200 & 0.368400 \\ \mathrm{C} & -5.071200 & -0.972000 & -0.712400 \\ \mathrm{H} & -5.840000 & -0.554800 & -1.364700 \\ \mathrm{H} & -5.239500 & -0.640800 & 0.318200 \\ \mathrm{H} & -5.108300 & -2.064500 & -0.731000 \\ \mathrm{O} & 2.099000 & 1.301600 & -0.537400 \\ \mathrm{Si} & 3.283300 & 0.079400 & -0.550500 \\ \mathrm{C} & 3.687000 & -0.319400 & -2.319600 \\ \mathrm{H} & 4.082700 & 0.558000 & -2.842100 \\ \mathrm{H} & 2.792000 & -0.659100 & -2.850400 \\ \mathrm{H} & 4.437100 & -1.115400 & -2.378000 \\ \mathrm{C} & 4.750500 & 0.727700 & 0.398700 \\ \mathrm{H} & 5.181500 & 1.602400 & -0.100900 \\ \mathrm{H} & 5.531700 & -0.036100 & 0.477100 \\ \mathrm{H} & 4.467900 & 1.026500 & 1.413200\end{array}$


Calculated energy for 8-cis:

$M P 2 / 6-31+G(d)=-389.322412$

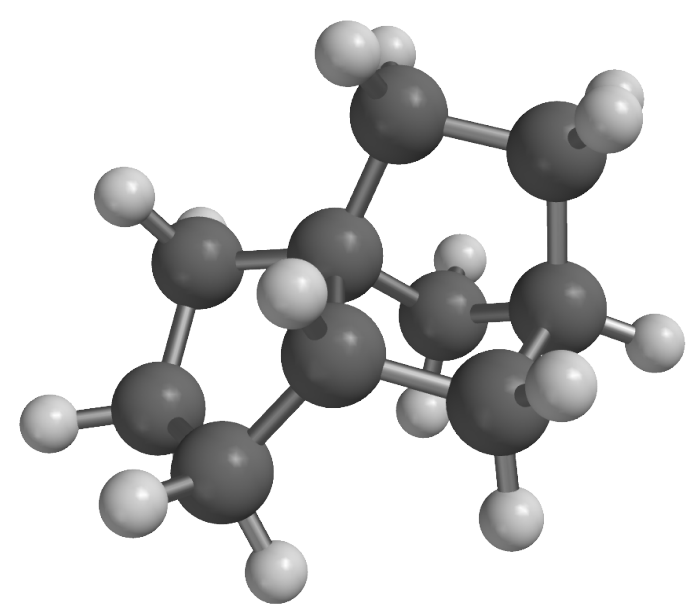

26

$\begin{array}{llll}\mathrm{H} & 0.665372 & -0.882775 & -2.717967\end{array}$

$\begin{array}{llll}\text { C } & 0.172210 & -0.592508 & -1.783157\end{array}$

$\begin{array}{llll}\text { C } & -0.245385 & 1.042236 & -0.056046\end{array}$

$\begin{array}{llll}\text { C } & -1.474638 & -1.145928 & -0.071319\end{array}$

$\begin{array}{llll}\text { C } & -0.222638 & -0.424692 & 0.435139\end{array}$

$\begin{array}{llll}\text { C } & -1.236965 & -1.201516 & -1.611109\end{array}$

$\begin{array}{llll}\text { C } & 0.094196 & 0.930586 & -1.567437\end{array}$

$\begin{array}{llll}\mathrm{H} & -1.542260 & -2.153861 & 0.359124\end{array}$

$\mathrm{H} \quad-1.992363 \quad-0.640946 \quad-2.174659$

$\begin{array}{llll}\mathrm{H} & -0.656934 & 1.405508 & -2.210478\end{array}$

$\begin{array}{llll}\mathrm{H} & -1.250694 & 1.462996 & 0.086275\end{array}$

$\begin{array}{llll}H & -2.391598 & -0.609206 & 0.203016\end{array}$

$\mathrm{H} \quad-1.259146 \quad-2.234613 \quad-1.977573$

$\begin{array}{llll}\mathrm{H} & 1.060798 & 1.401390 & -1.791300\end{array}$

$\begin{array}{llll}\text { C } & 0.857071 & -1.033193 & -0.482002\end{array}$

$\begin{array}{llll}\mathrm{H} & 1.850632 & -0.587145 & -0.349022\end{array}$

H $\quad 0.938619 \quad-2.122930 \quad-0.370683$

$\begin{array}{llll}\text { C } & 0.061832 & -0.333058 & 1.922084\end{array}$

$\begin{array}{llll}\mathrm{H} & 0.419684 & -1.276875 & 2.352811\end{array}$

H $\quad-0.862264 \quad-0.058545 \quad 2.451625$

$\begin{array}{llll}\text { C } & 0.756210 & 1.781160 & 0.865126\end{array}$

$\begin{array}{llll}H & 1.660884 & 2.071489 & 0.317007\end{array}$

$\begin{array}{llll}\mathrm{H} & 0.315975 & 2.708413 & 1.250238\end{array}$

$\begin{array}{llll}\text { C } & 1.095970 & 0.803122 & 2.023750\end{array}$

$\begin{array}{llll}\mathrm{H} & 2.105094 & 0.395017 & 1.894288\end{array}$

$\begin{array}{llll}H & 1.080337 & 1.295874 & 3.002271\end{array}$

Calculated energy for 9-trans: 


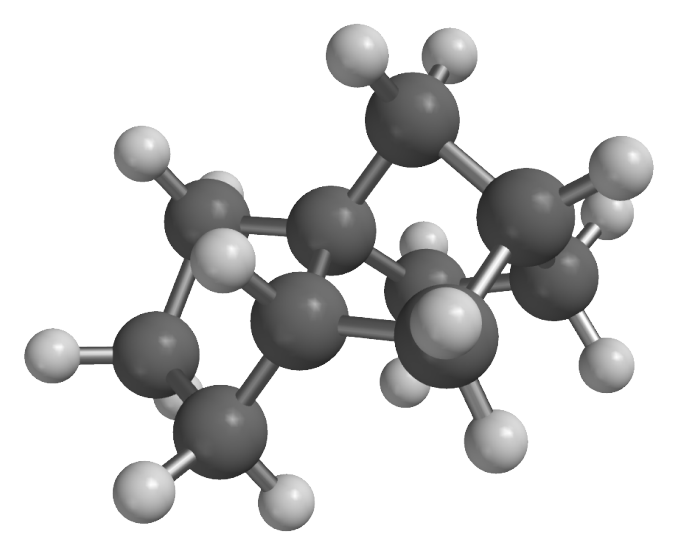

26

$\begin{array}{lrrr}\mathrm{H} & 0.874179 & 0.248299 & -2.203387 \\ \mathrm{C} & 0.920077 & 0.327629 & -1.109418 \\ \mathrm{C} & -0.099842 & 1.991523 & 0.289275 \\ \mathrm{C} & -0.310379 & -0.296770 & 1.007089 \\ \mathrm{C} & 0.145728 & 1.080940 & 1.527404 \\ \mathrm{C} & -0.444065 & -0.086686 & -0.507776 \\ \mathrm{C} & 1.101320 & 1.799448 & -0.648294 \\ \mathrm{H} & 1.046682 & 2.490800 & -1.498358 \\ \mathrm{H} & -1.332832 & -0.477825 & 1.377691 \\ \mathrm{H} & 2.057113 & 1.976938 & -0.141330 \\ \mathrm{H} & 1.739420 & -0.320328 & -0.779030 \\ \mathrm{H} & 1.203999 & 1.097819 & 1.820522 \\ \mathrm{H} & -0.308382 & 3.039343 & 0.535564 \\ \mathrm{H} & -0.437442 & 1.399202 & 2.399675 \\ \mathrm{C} & -1.198349 & 1.246821 & -0.516220 \\ \mathrm{H} & -2.167799 & 1.185948 & -0.004845 \\ \mathrm{H} & -1.346323 & 1.688127 & -1.510915 \\ \mathrm{C} & -0.928725 & -1.450525 & -0.964966 \\ \mathrm{H} & -0.805790 & -1.627253 & -2.040603 \\ \mathrm{H} & -1.995282 & -1.569236 & -0.729747 \\ \mathrm{C} & 0.446851 & -1.616351 & 1.131413 \\ \mathrm{H} & 1.531307 & -1.460014 & 1.083672 \\ \mathrm{H} & 0.244237 & -2.133124 & 2.076360 \\ \mathrm{C} & -0.065248 & -2.426806 & -0.104711 \\ \mathrm{H} & 0.779222 & -2.806519 & -0.691163 \\ \mathrm{H} & -0.649678 & -3.301400 & 0.202097\end{array}$




\section{Reproducing the calculated ECD data from the isolation paper:}

(Ma, S.-G.; Li, M.; Lin, M.-B.; Li, L.; Liu, Y.-B.; Qu, J.; Li, Y.; Wang, X.-J.; Wang, R.-B.; Xu, S.; Hou, Q.; Yu, S.-S. Illisimonin A, a Caged Sesquiterpenoid with a Tricyclo[5.2.1.0 1,6]Decane Skeleton From the Fruits of Illicium Simonsii. Org. Lett. 2017, 19, 6160-6163.)

These sentences describe the computational procedure used the isolation paper authors. There was no additional information in the experimental or SI for the paper.

"Conformational analysis of two possible enantiomers of 1 was performed using the MMFF94 molecular mechanics force field. The conformers were further optimized using Gaussian 03 at the B3LYP/6-31 G(d) level. The theoretical ECD spectra of 1 and its enantiomer in MeOH were then compared to the experimental ECD spectrum of $1 . "$

These steps were repeated to generate the following structure and conformation of illisimonin $A$ with the assigned 1R configuration. The various conformations differed only in the $\mathrm{H}$-bond patterns. The optimized B3LYP/6-31 G(d) (geometry included below) was used to calculate the ECD spectrum using the "TD" keyword in Gaussian 16.

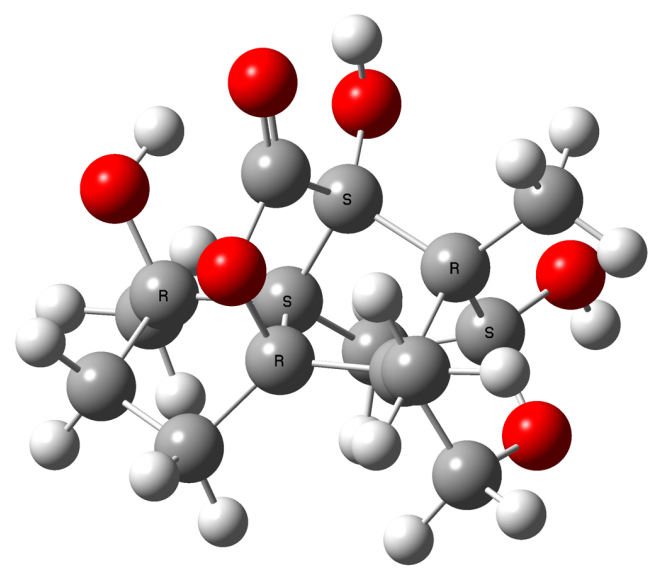

$E(R B 3 L Y P)=-1034.94749110$

41

C $\quad-0.7103129$

$-0.3745869$

$-0.3073488$

C $\quad-0.3610069$

0.4583831

0.9611692

C $\quad-1.3046799$

$-0.0367989 \quad 2.0390332$

C $\quad-2.6267409$

$-0.2504009 \quad 1.2491092$

C $\quad-2.2615739$

$-0.5378419 \quad-0.2506188$

H $\quad-0.9353309$

$-0.9825399$

2.4518002

H $\quad-1.4162709$

$0.6691701 \quad 2.8673692$

H $\quad-3.2285149$

$0.6613541 \quad 1.2645722$

H $\quad-3.2296209$

$-1.0555139 \quad 1.6805982$

C $\quad-0.0248609$

0.6586141

$-1.2228218$

C $\quad 1.4242791$

0.4358241

$-0.6149268$

C $\quad 1.5947031$

$-1.1091879$

$-0.6563248$

C $\quad 0.1413171$

$-1.6422049$

$-0.4860778$ 


$\begin{array}{rrrr}\mathrm{H} & 0.0680631 & -2.3581879 & 0.3391242 \\ \mathrm{H} & -0.1635659 & -2.1494219 & -1.4057768 \\ \mathrm{C} & 1.1964141 & 0.5770641 & 0.9596392 \\ \mathrm{C} & 1.7190741 & 1.8575351 & 1.6229382 \\ \mathrm{H} & 2.8072681 & 1.9174181 & 1.5177372 \\ \mathrm{H} & 1.4894061 & 1.8477821 & 2.6957722 \\ \mathrm{H} & 1.2822061 & 2.7651381 & 1.2037682 \\ \mathrm{C} & 1.9276251 & -0.6608229 & 1.5569832 \\ \mathrm{H} & 1.2665471 & -1.2511479 & 2.2082812 \\ \mathrm{H} & 2.7971881 & -0.3646499 & 2.1510712 \\ \mathrm{O} & 2.3987371 & -1.4477399 & 0.4692622 \\ \mathrm{C} & 2.5483981 & 1.2251681 & -1.2581938 \\ \mathrm{H} & 2.4201761 & 2.3024421 & -1.1070868 \\ \mathrm{H} & 2.5698141 & 1.0255641 & -2.3332428 \\ \mathrm{H} & 3.5185131 & 0.9290551 & -0.8464468 \\ \mathrm{C} & -2.7761369 & -1.9058329 & -0.7124168 \\ \mathrm{H} & -2.3792759 & -2.7267899 & -0.1051948 \\ \mathrm{H} & -2.5115649 & -2.0998289 & -1.7589328 \\ \mathrm{H} & -3.8677979 & -1.9153209 & -0.6324708 \\ \mathrm{O} & -2.9050189 & 0.4791741 & -1.0337188 \\ \mathrm{H} & -2.5978999 & 0.3688931 & -1.9503148 \\ \mathrm{O} & -0.1594539 & 0.5193851 & -2.6090488 \\ \mathrm{H} & -0.2175809 & 1.4301081 & -2.9579478 \\ \mathrm{C} & -0.5814099 & 1.9709231 & -0.7189638 \\ \mathrm{O} & -0.7402789 & 2.9829951 & -1.3590518 \\ \mathrm{O} & 2.2399481 & -1.5412749 & -1.8083738 \\ \mathrm{H} & 2.4198641 & -2.4900729 & -1.7017608 \\ \mathrm{O} & -0.8306459 & 1.8181761 & 0.5988342\end{array}$

Calculated ECD spectrum has a positive sign at $214 \mathrm{~nm}$. It appears to have the opposite sign to the one reported by the authors of the isolation paper.

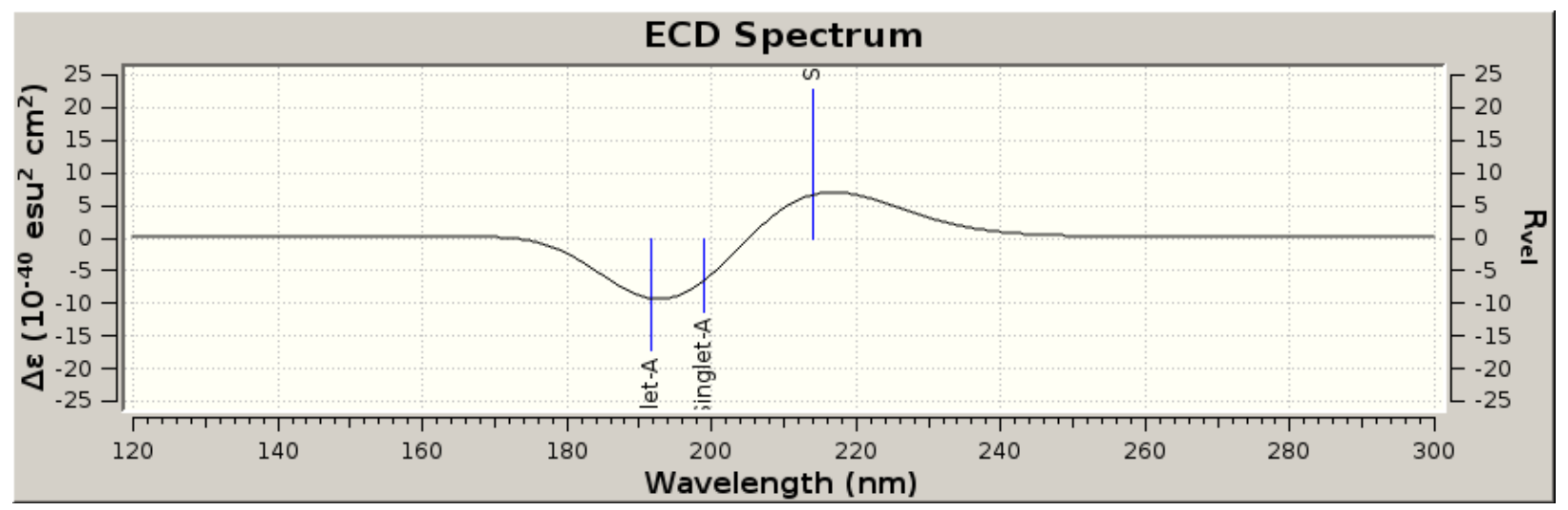




\section{Computation References:}

${ }^{1}$ Gaussian 16, Revision A.03, M. J. Frisch, G. W. Trucks, H. B. Schlegel, G. E. Scuseria, M. A. Robb, J. R. Cheeseman, G. Scalmani, V. Barone, G. A. Petersson, H. Nakatsuji, X. Li, M. Caricato, A. V. Marenich, J. Bloino, B. G. Janesko, R. Gomperts, B. Mennucci, H. P. Hratchian, J. V. Ortiz, A. F. Izmaylov, J. L. Sonnenberg, D. Williams-Young, F. Ding, F. Lipparini, F. Egidi, J. Goings, B. Peng, A. Petrone, T. Henderson, D. Ranasinghe, V. G. Zakrzewski, J. Gao, N. Rega, G. Zheng, W. Liang, M. Hada, M. Ehara, K. Toyota, R. Fukuda, J. Hasegawa, M. Ishida, T. Nakajima, Y. Honda, O. Kitao, H. Nakai, T. Vreven, K. Throssell, J. A. Montgomery, Jr., J. E. Peralta, F. Ogliaro, M. J. Bearpark, J. J. Heyd, E. N. Brothers, K. N. Kudin, V. N. Staroverov, T. A. Keith, R. Kobayashi, J. Normand, K. Raghavachari, A. P. Rendell, J. C. Burant, S. S. Iyengar, J. Tomasi, M. Cossi, J. M. Millam, M. Klene, C. Adamo, R. Cammi, J. W. Ochterski, R. L. Martin, K. Morokuma, O. Farkas, J. B. Foresman, and D. J. Fox, Gaussian, Inc., Wallingford CT, 2016.

2 (a) Spartan 1, Wavefunction Inc., Irvine CA. (b) Shao, Y.; Gan, Z.; Epifanovsky, E.; Gilbert, A. T. B.; Wormit, M.; Kussmann, J.; Lange, A. W.; Behn, A.; Deng, J.; Feng, X.; Ghosh, D.; Goldey, M.; Horn, P. R.; Jacobson, L. D.; Kaliman, I.; Khaliullin, R. Z.; Kuś, T.; Landau, A.; Liu, J.; Proynov, E. I.; Rhee, Y. M.; Richard, R. M.; Rohrdanz, M. A.; Steele, R. P.; Sundstrom, E. J.; Woodcock, H. L., III; Zimmerman, P. M.; Zuev, D.; Albrecht, B.; Alguire, E.; Austin, B.; Beran, G. J. O.; Bernard, Y. A.; Berquist, E.; Brandhorst, K.; Bravaya, K. B.; Brown, S. T.; Casanova, D.; Chang, C.-M.; Chen, Y.; Chien, S. H.; Closser, K. D.; Crittenden, D. L.; Diedenhofen, M.; DiStasio, R. A., Jr.; Do, H.; Dutoi, A. D.; Edgar, R. G.; Fatehi, S.; Fusti-Molnar, L.; Ghysels, A.; Golubeva-Zadorozhnaya, A.; Gomes, J.; Hanson-Heine, M. W. D.; Harbach, P. H. P.; Hauser, A. W.; Hohenstein, E. G.; Holden, Z. C.; Jagau, T.-C.; Ji, H.; Kaduk, B.; Khistyaev, K.; Kim, J.; Kim, J.; King, R. A.; Klunzinger, P.; Kosenkov, D.; Kowalczyk, T.; Krauter, C. M.; Lao, K. U.; Laurent, A. D.; Lawler, K. V.; Levchenko, S. V.; Lin, C. Y.; Liu, F.; Livshits, E.; Lochan, R. C.; Luenser, A.; Manohar, P.; Manzer, S. F.; Mao, S.-P.; Mardirossian, N.; Marenich, A. V.; Maurer, S. A.; Mayhall, N. J.; Neuscamman, E.; Oana, C. M.; Olivares-Amaya, R.; O’Neill, D. P.; Parkhill, J. A.; Perrine, T. M.; Peverati, R.; Prociuk, A.; Rehn, D. R.; Rosta, E.; Russ, N. J.; Sharada, S. M.; Sharma, S.; Small, D. W.; Sodt, A.; Stein, T.; Stück, D.; Su, Y.-C.; Thom, A. J. W.; Tsuchimochi, T.; Vanovschi, V.; Vogt, L.; Vydrov, O.; Wang, T.; Watson, M. A.; Wenzel, J.; White, A.; Williams, C. F.; Yang, J.; Yeganeh, S.; Yost, S. R.; You, Z.-Q.; Zhang, I. Y.; Zhang, X.; Zhao, Y.; Brooks, B. R.; Chan, G. K. L.; Chipman, D. M.; Cramer, C. J.; Goddard lii, W. A.; Gordon, M. S.; Hehre, W. J.; Klamt, A.; Schaefer, H. F., III; Schmidt, M. W.; Sherrill, C. D.; Truhlar, D. G.; Warshel, A.; Xu, X.; Aspuru-Guzik, A.; Baer, R.; Bell, A. T.; Besley, N. A.; Chai, J.-D.; Dreuw, A.; Dunietz, B. D.; Furlani, T. R.; Gwaltney, S. R.; Hsu, C.-P.; Jung, Y.; Kong, J.; Lambrecht, D. S.; Liang, W.; Ochsenfeld, C.; Rassolov, V. A.; Slipchenko, L. V.; Subotnik, J. E.; Van Voorhis, T.; Herbert, J. M.; Krylov, A. I.; Gill, P. M. W.; Head-Gordon, M. Advances in Molecular Quantum Chemistry Contained in the Q-Chem 4 Program Package. Molecular Physics 2014, 113, 184-215.

${ }^{3}$ CYLview, 1.0b; Legault, C. Y., Université de Sherbrooke, 2009 (http://www.cylview.org). 


${ }^{1} \mathrm{H} \mathrm{NMR,} 500 \mathrm{MHz}, \mathrm{CD}_{3} \mathrm{OD}$

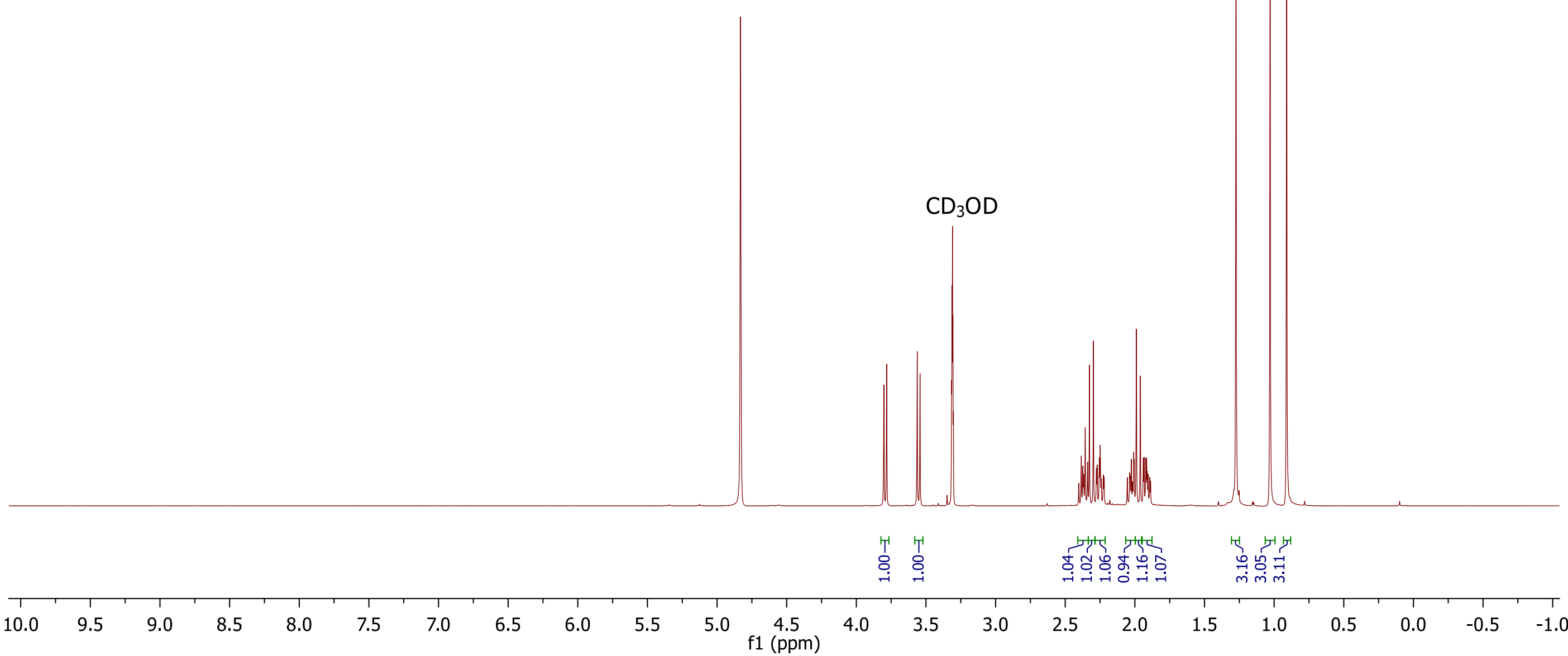



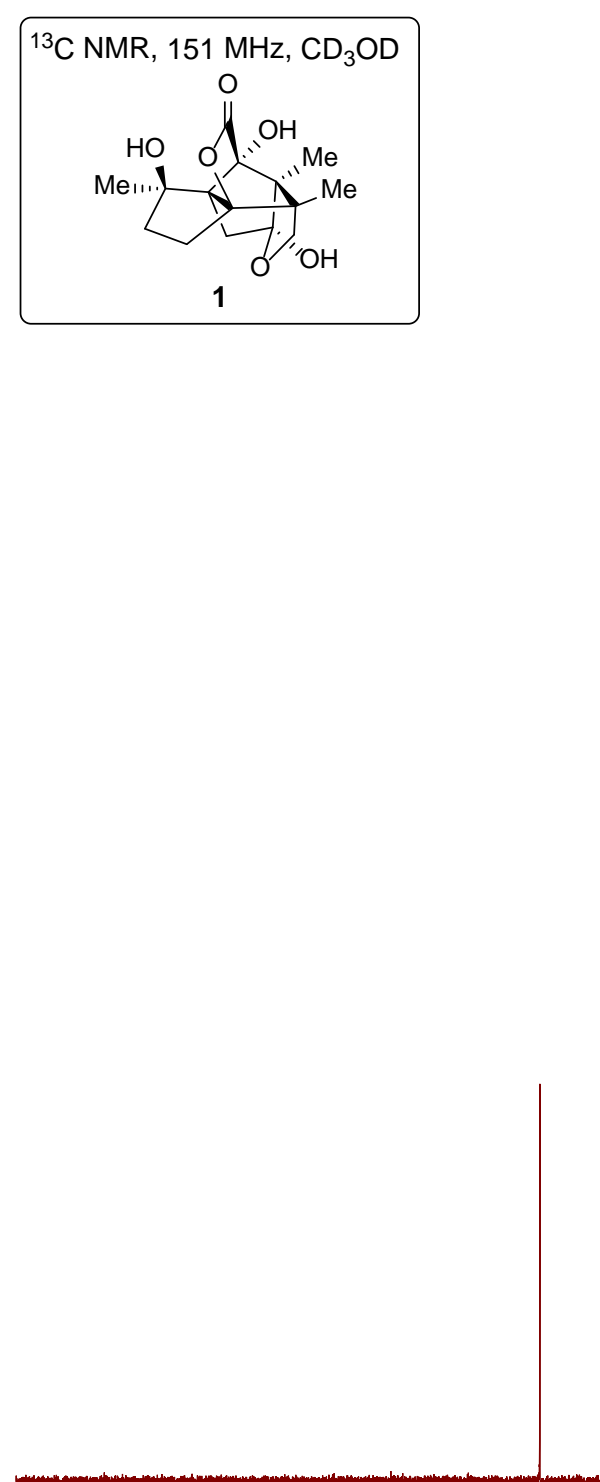


${ }_{7}^{{ }^{1} \mathrm{H} \mathrm{NMR}, 500 \mathrm{MHz}, \mathrm{CDCl}_{3}}$

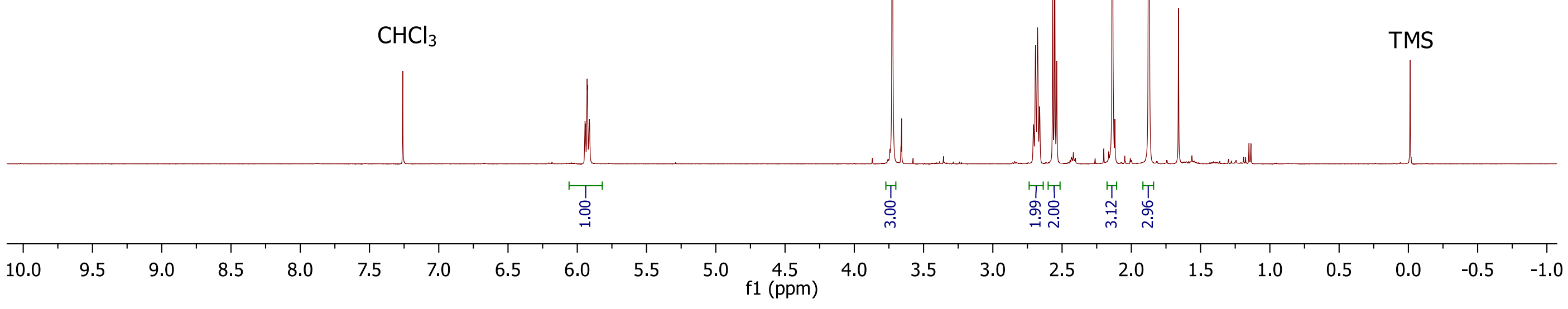



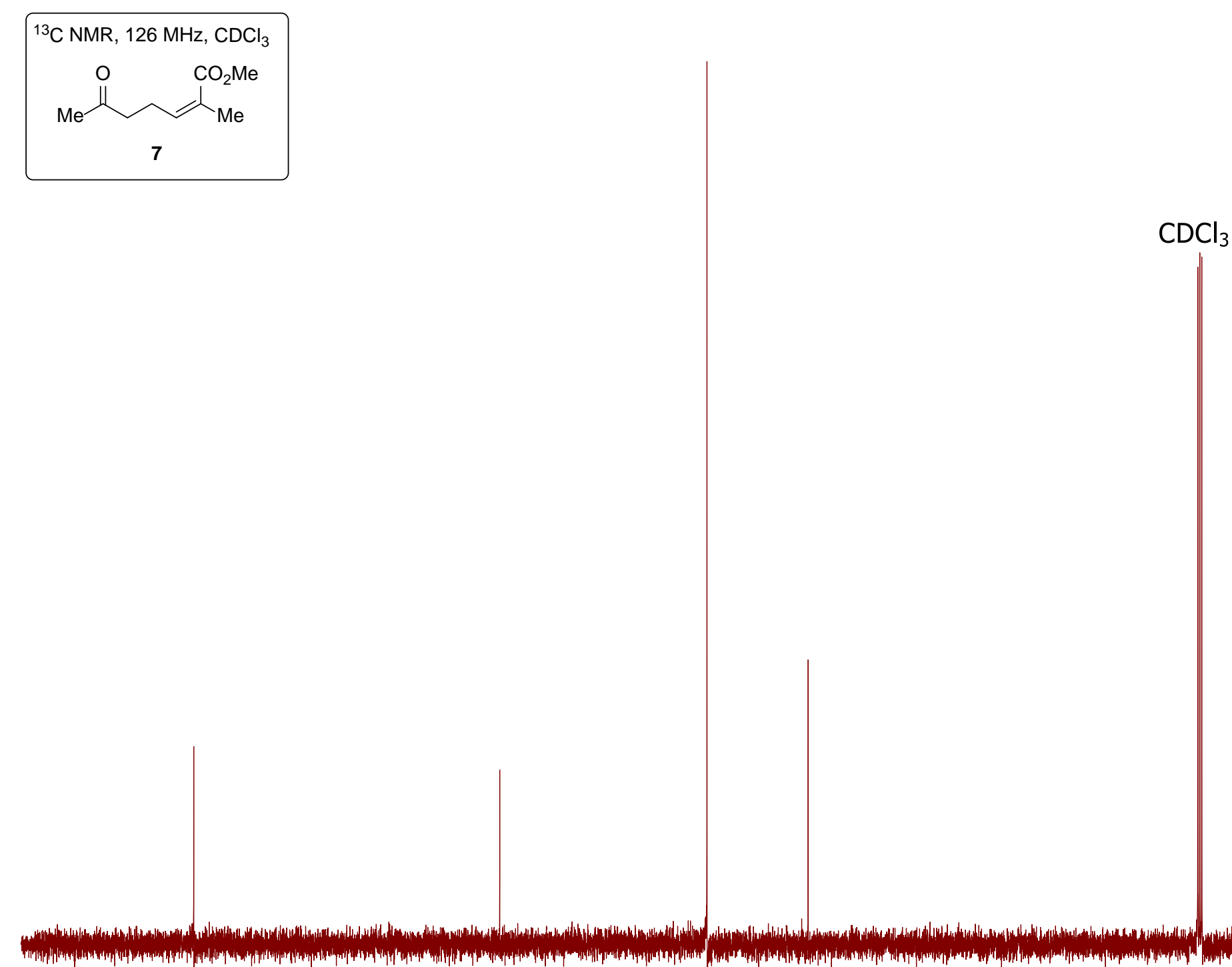

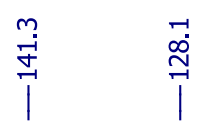

ঙं

ণิे

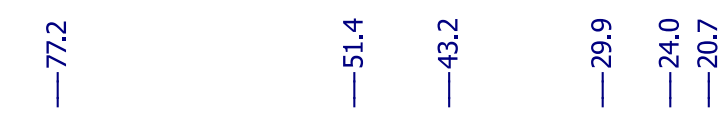

$\mathrm{CDCl}_{3}$

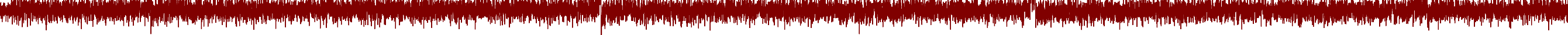

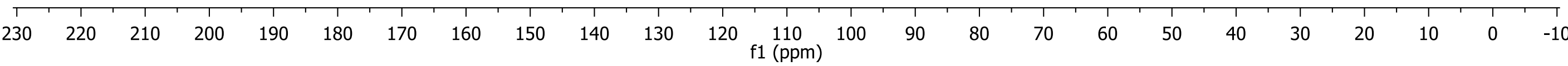




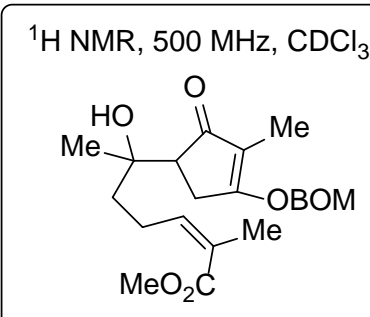

11 major diastereomer

11 major diastereomer

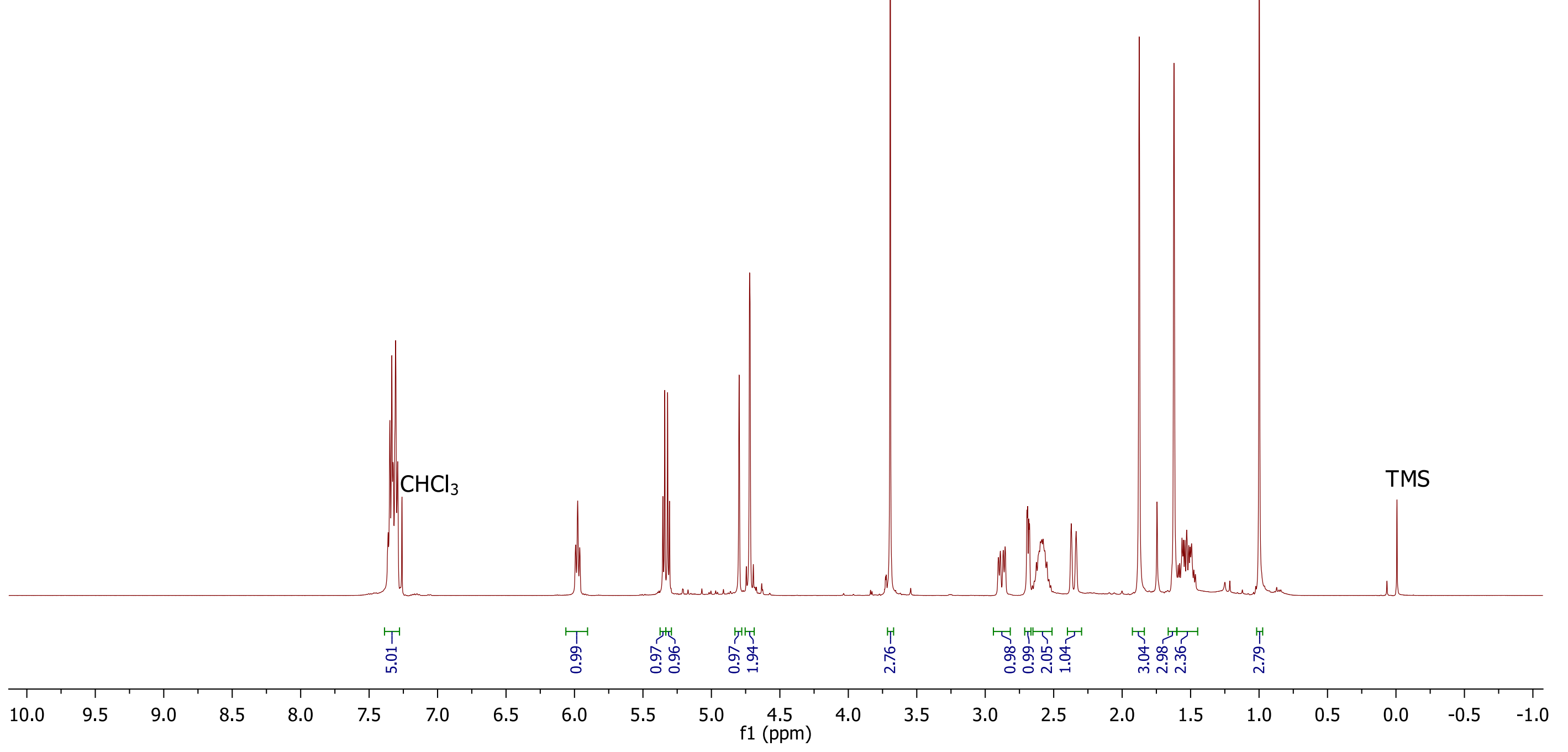




|

ก̊ำ

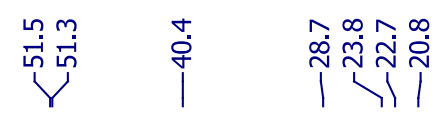

คे

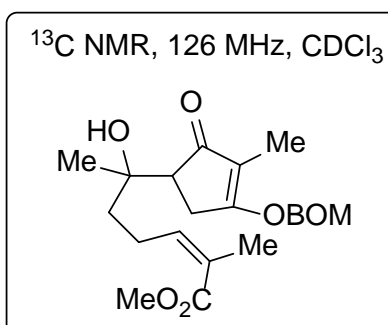

11 major diastereomer

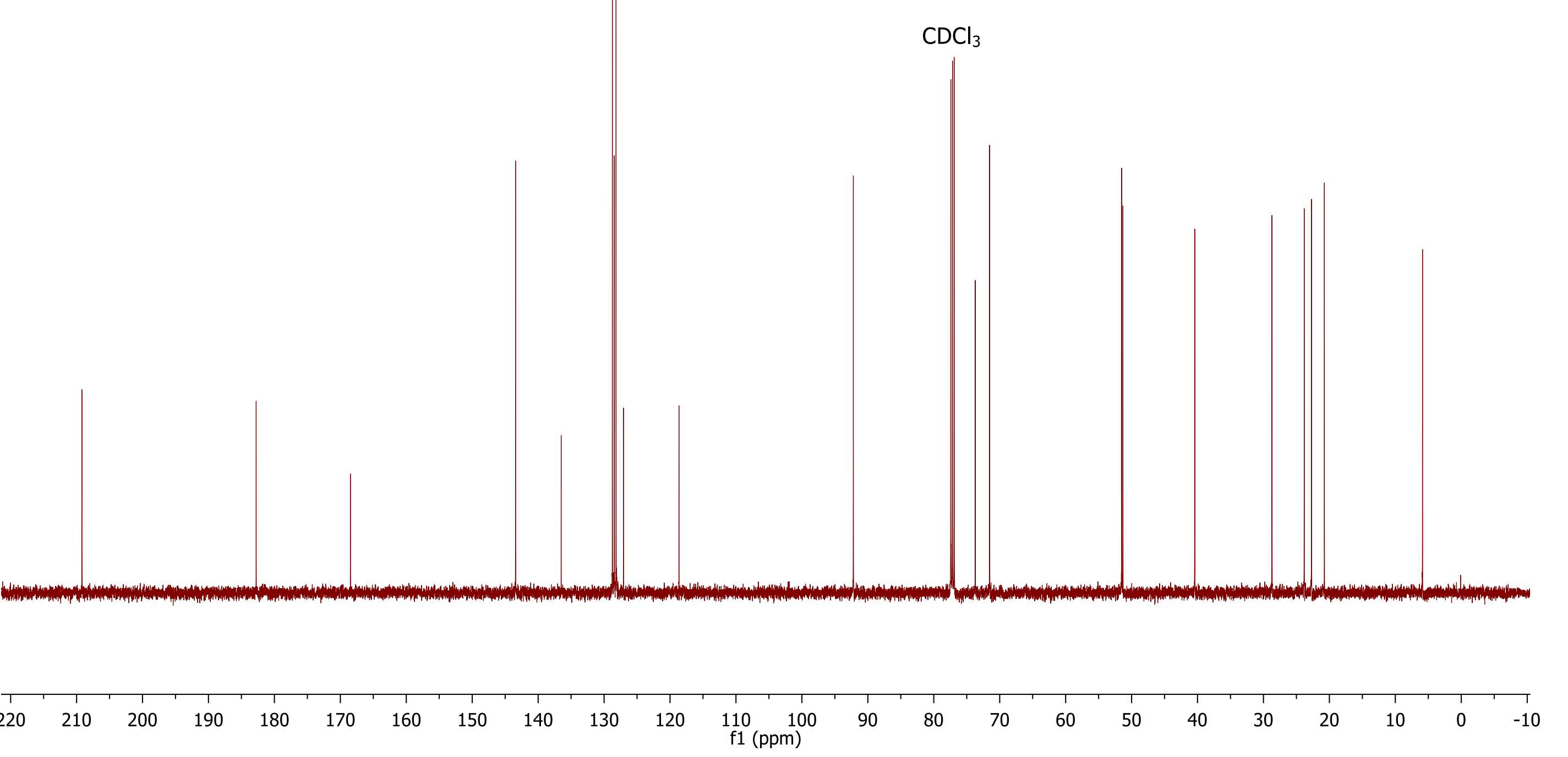




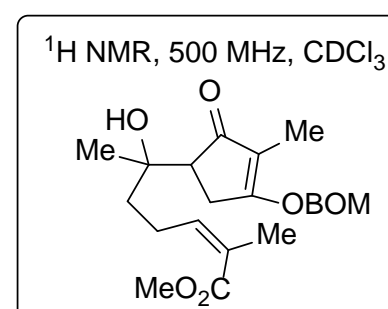

11 minor diastereomer

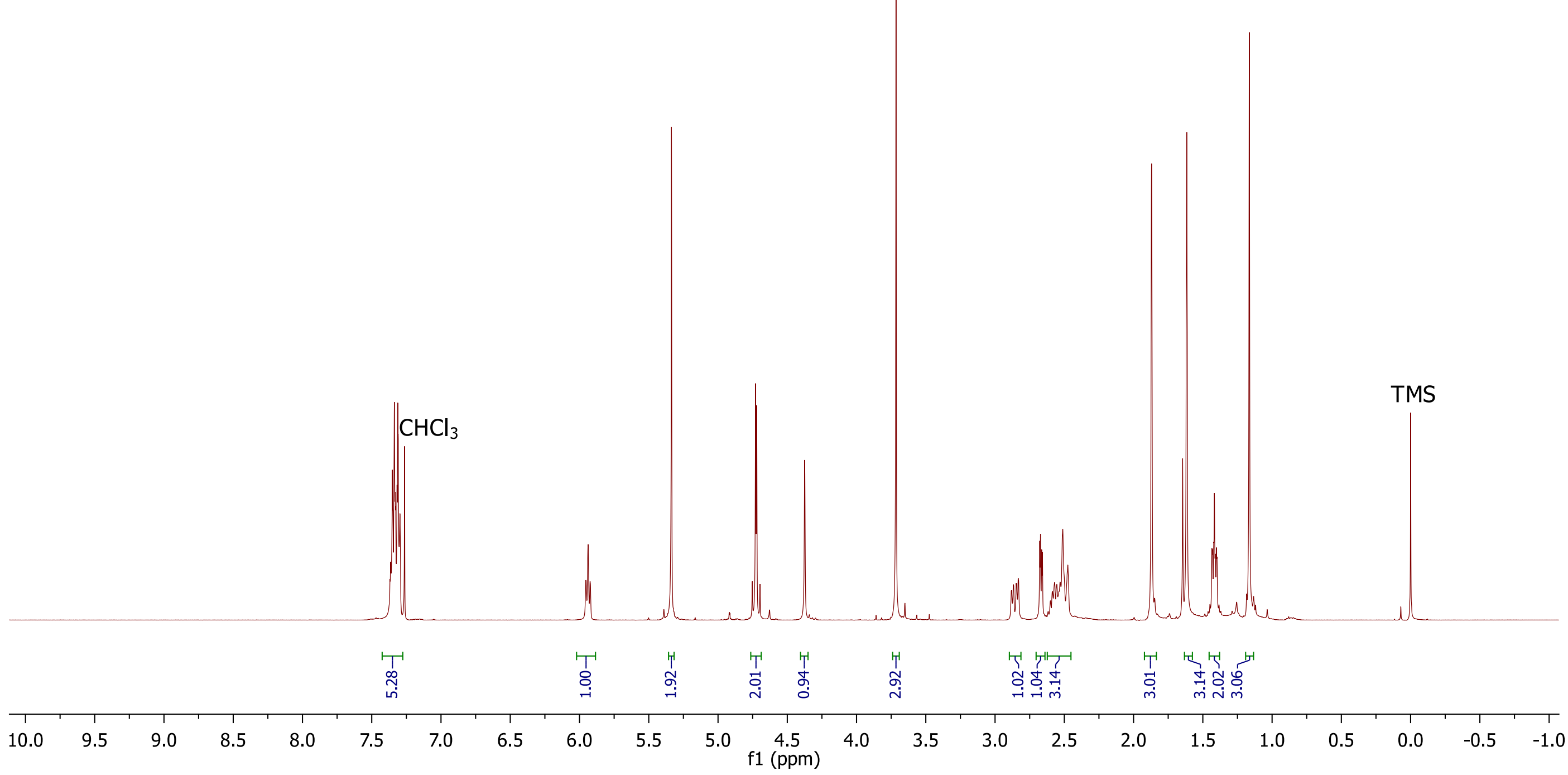




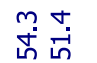

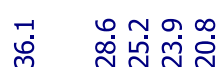

| । १रा

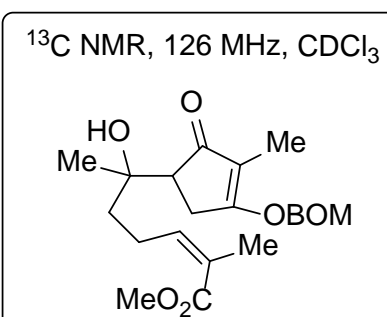

11 minor diastereomer

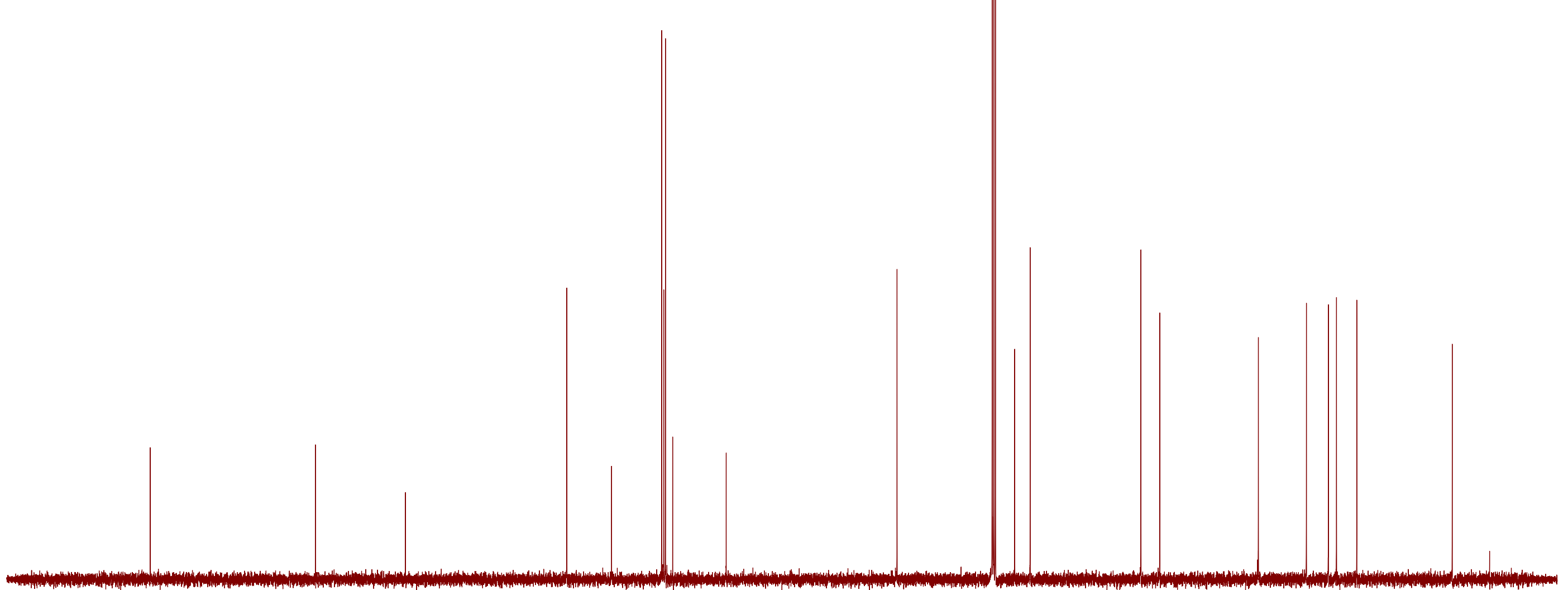


${ }^{1} \mathrm{H} \mathrm{NMR,}, 500 \mathrm{MHz}, \mathrm{CDCl}_{3}$

$$
\text { (13) }
$$

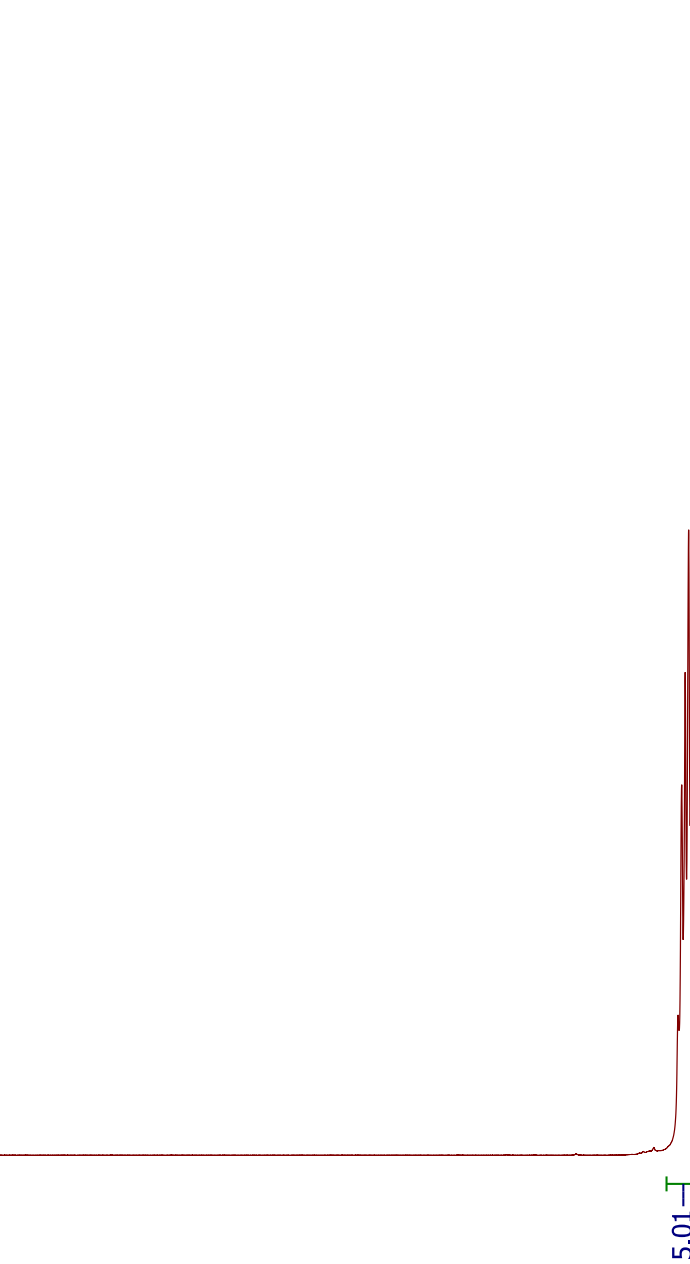

$\mathrm{CHCl}_{3}$
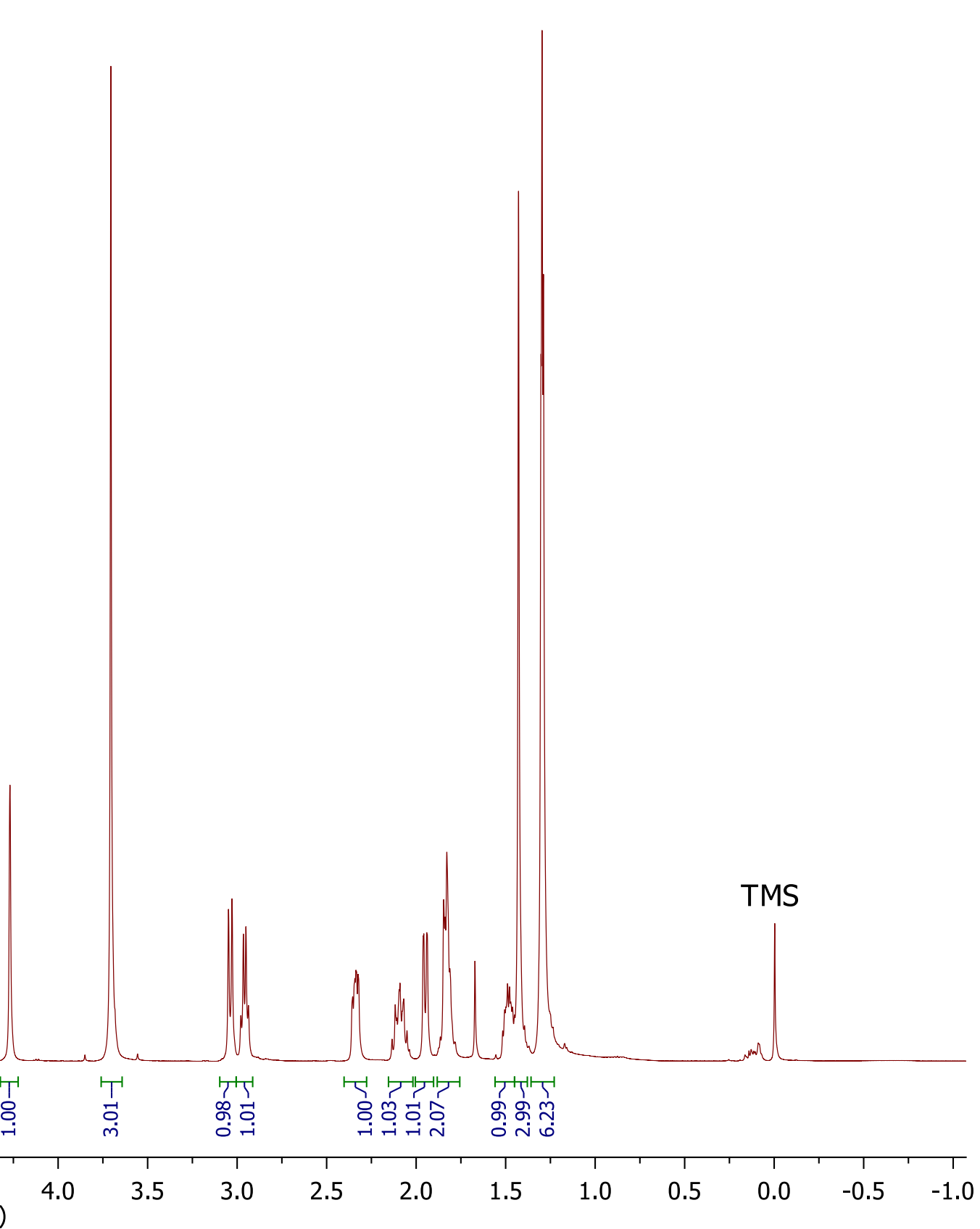

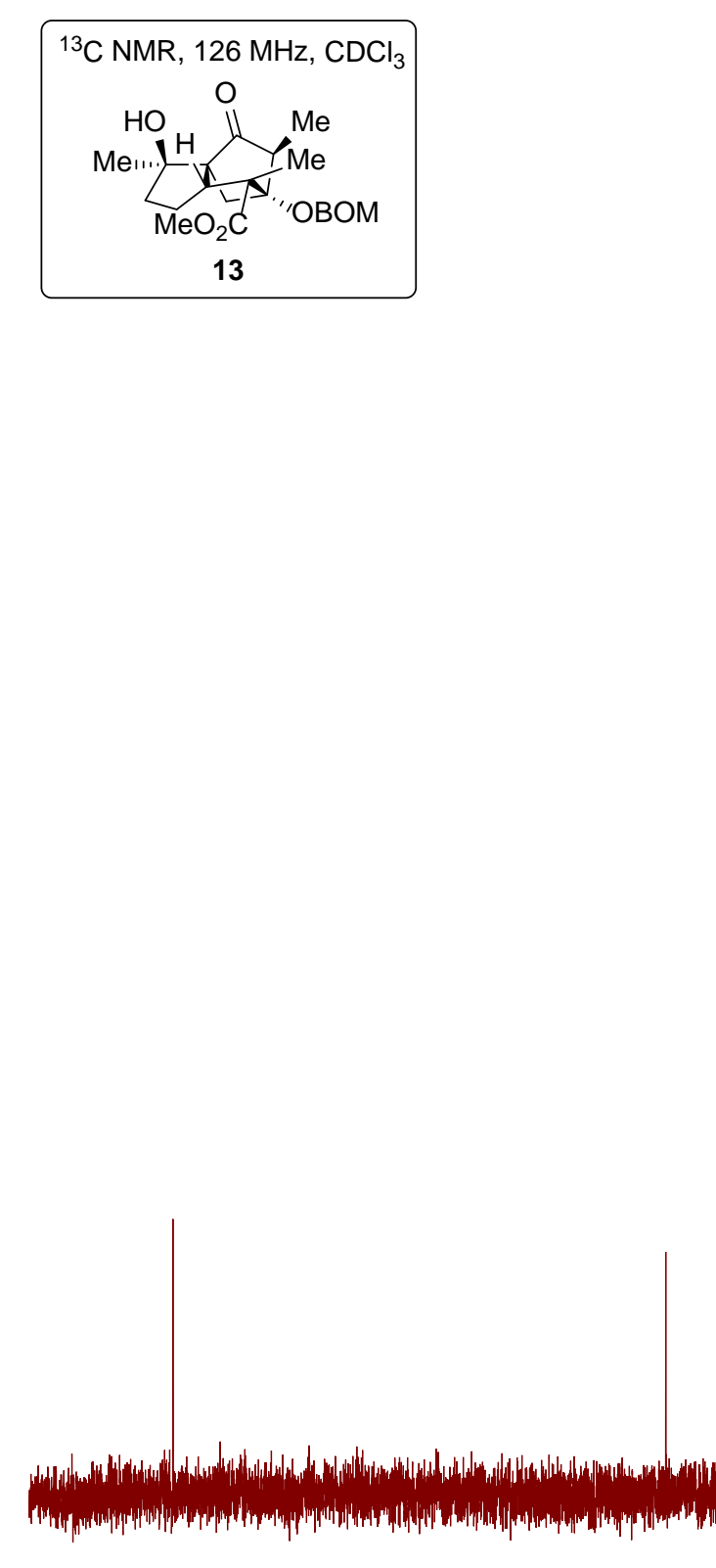


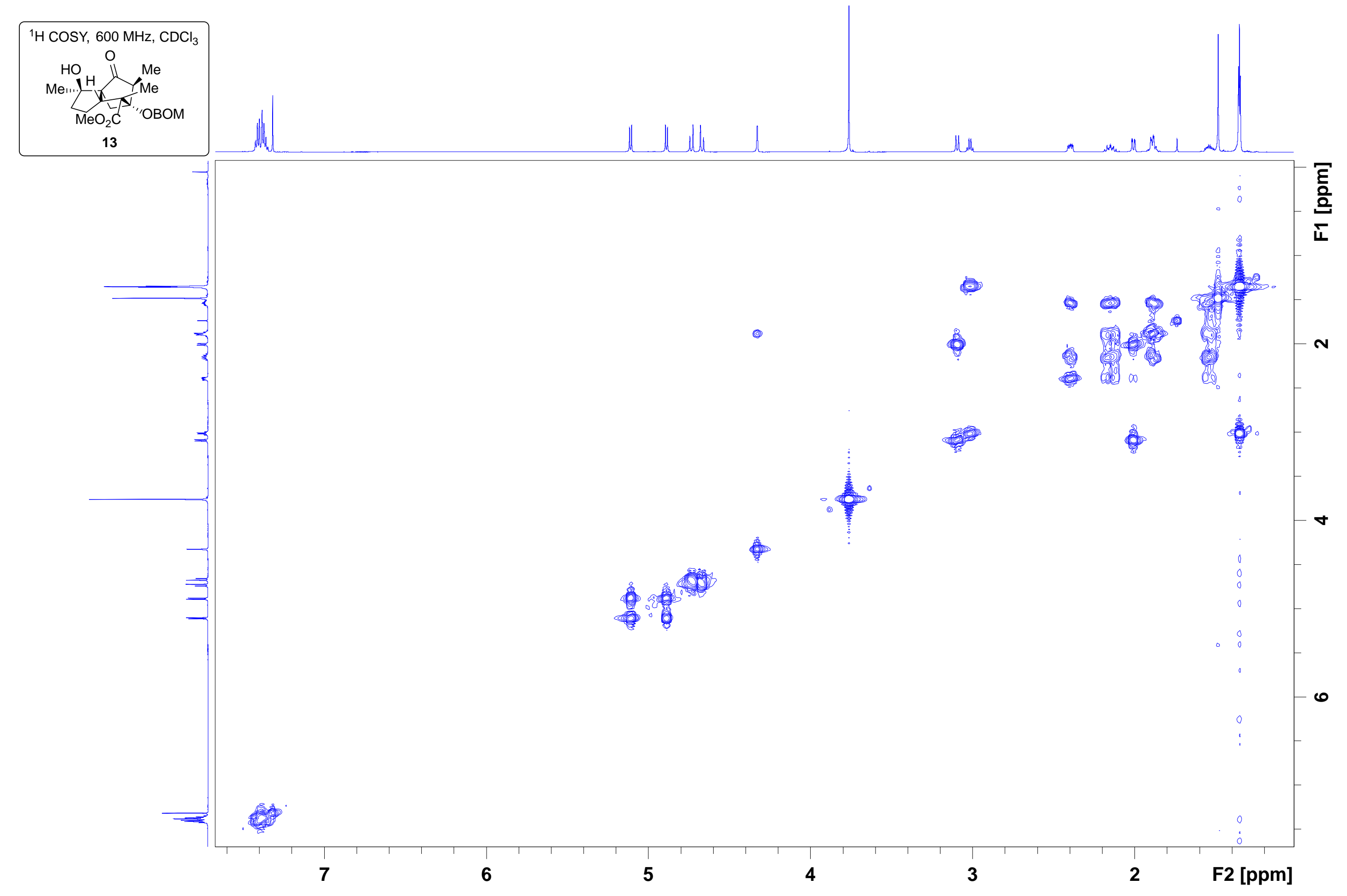




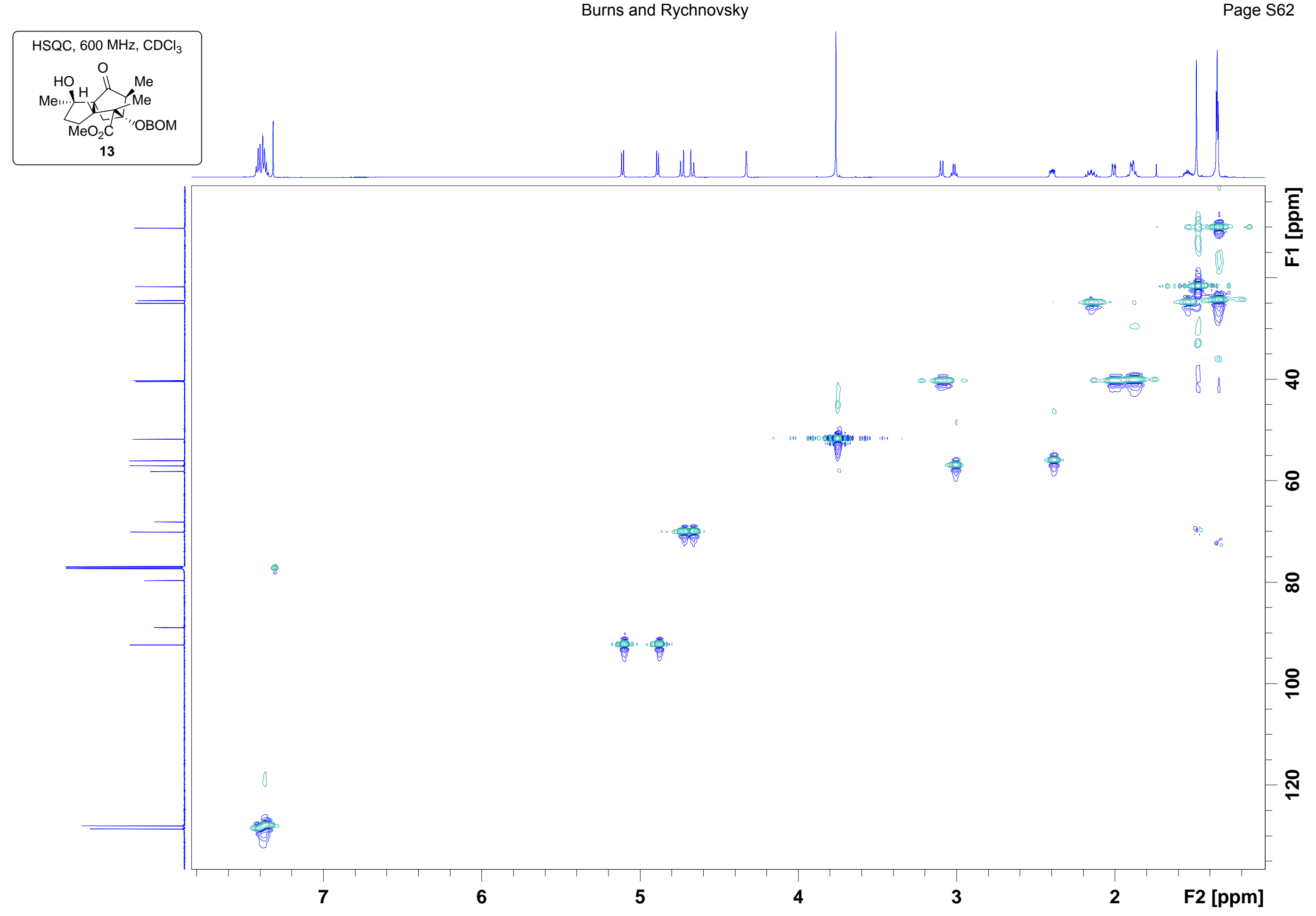




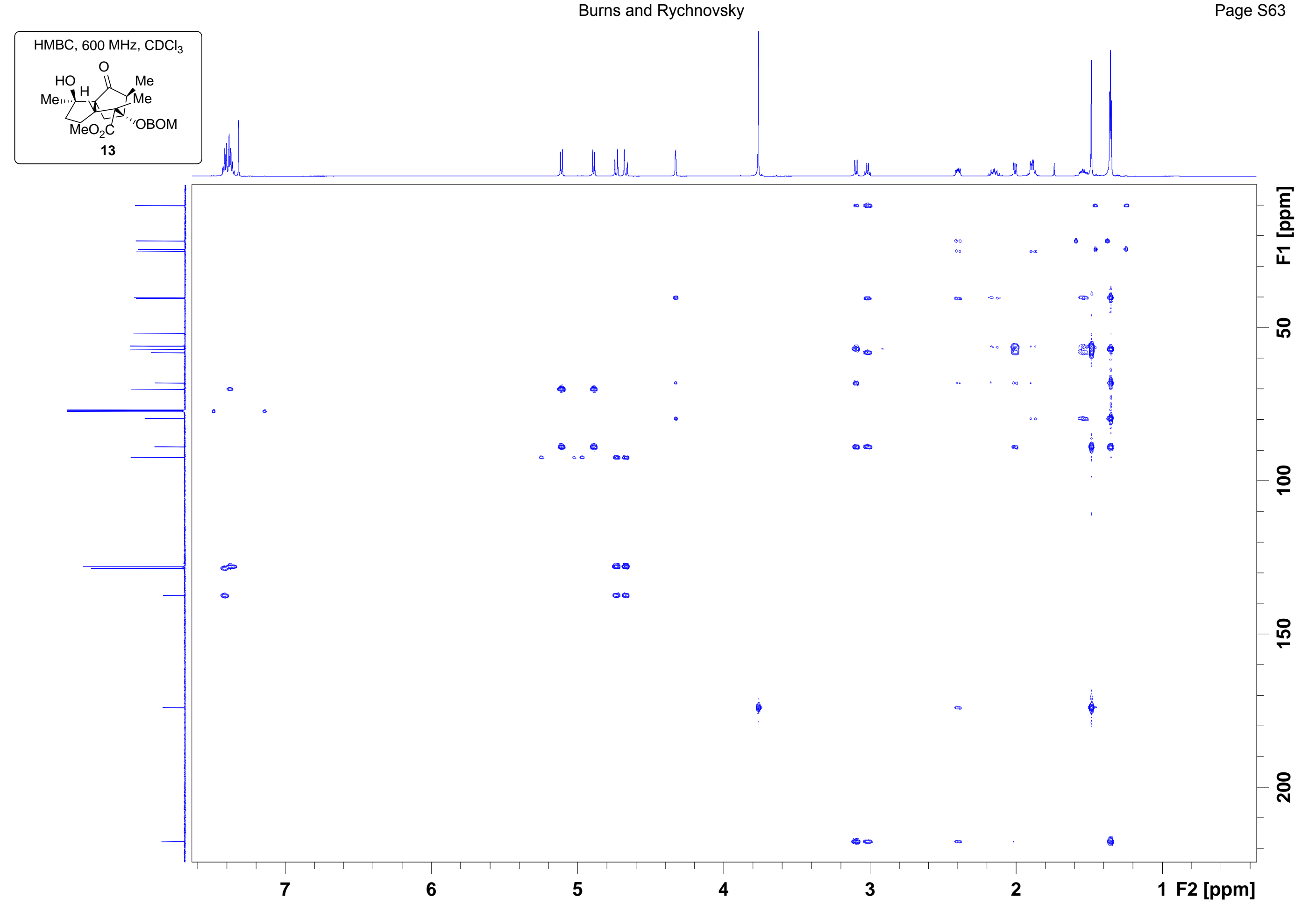




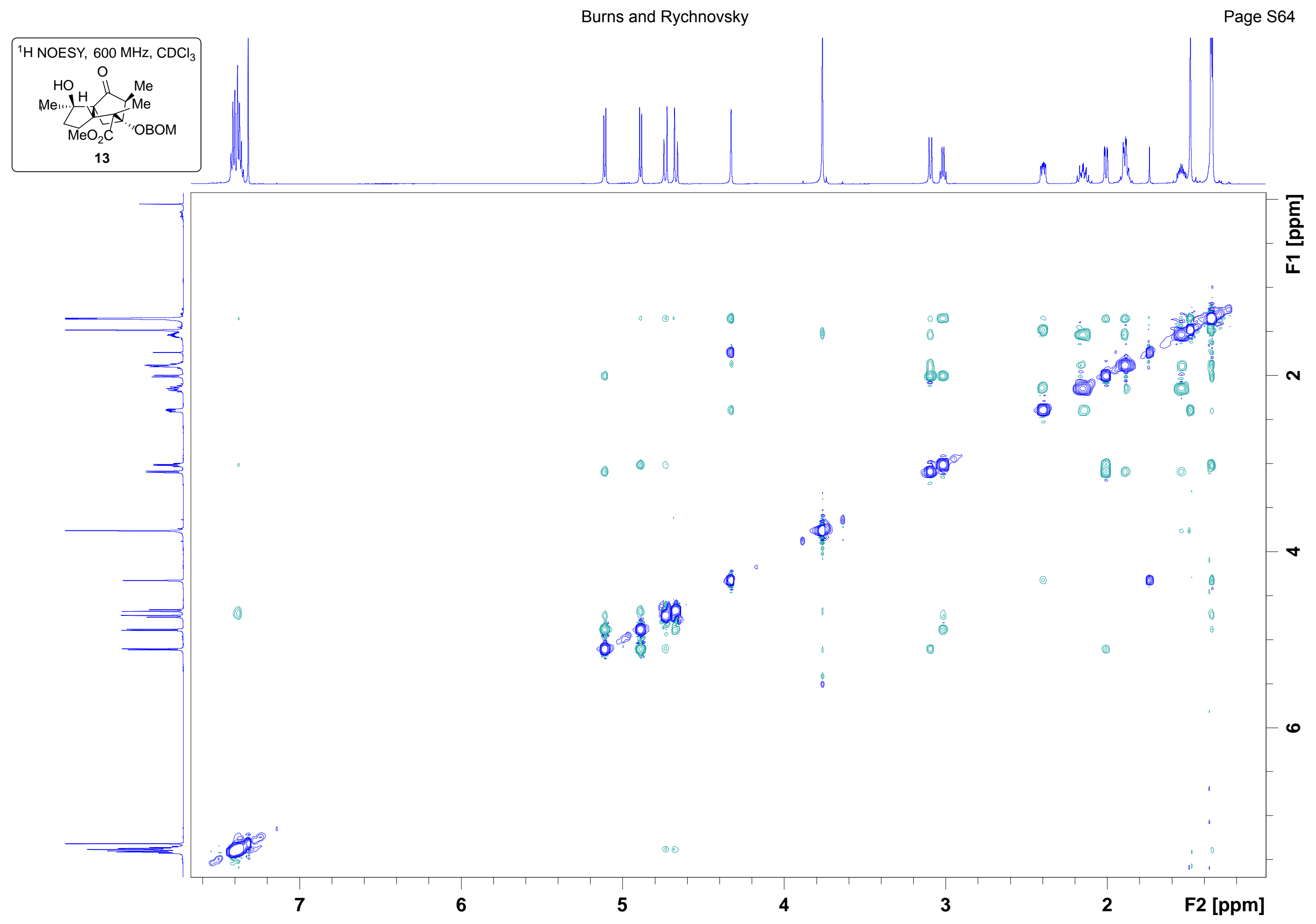




1H NMR, $500 \mathrm{MHz}, \mathrm{CDCl}_{3}$

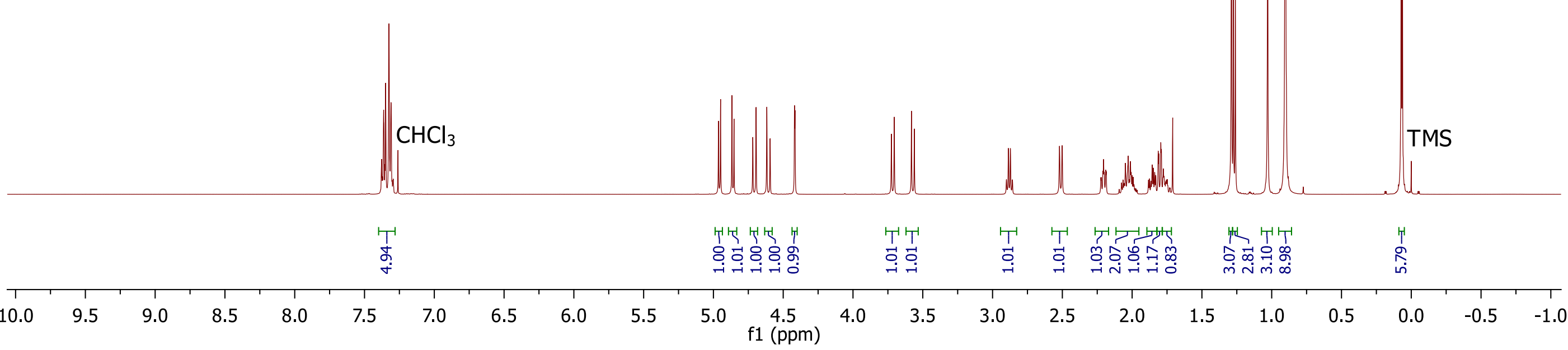


${ }^{13} \mathrm{CNMR}, 126 \mathrm{MHz}, \mathrm{CDCl}_{3}$
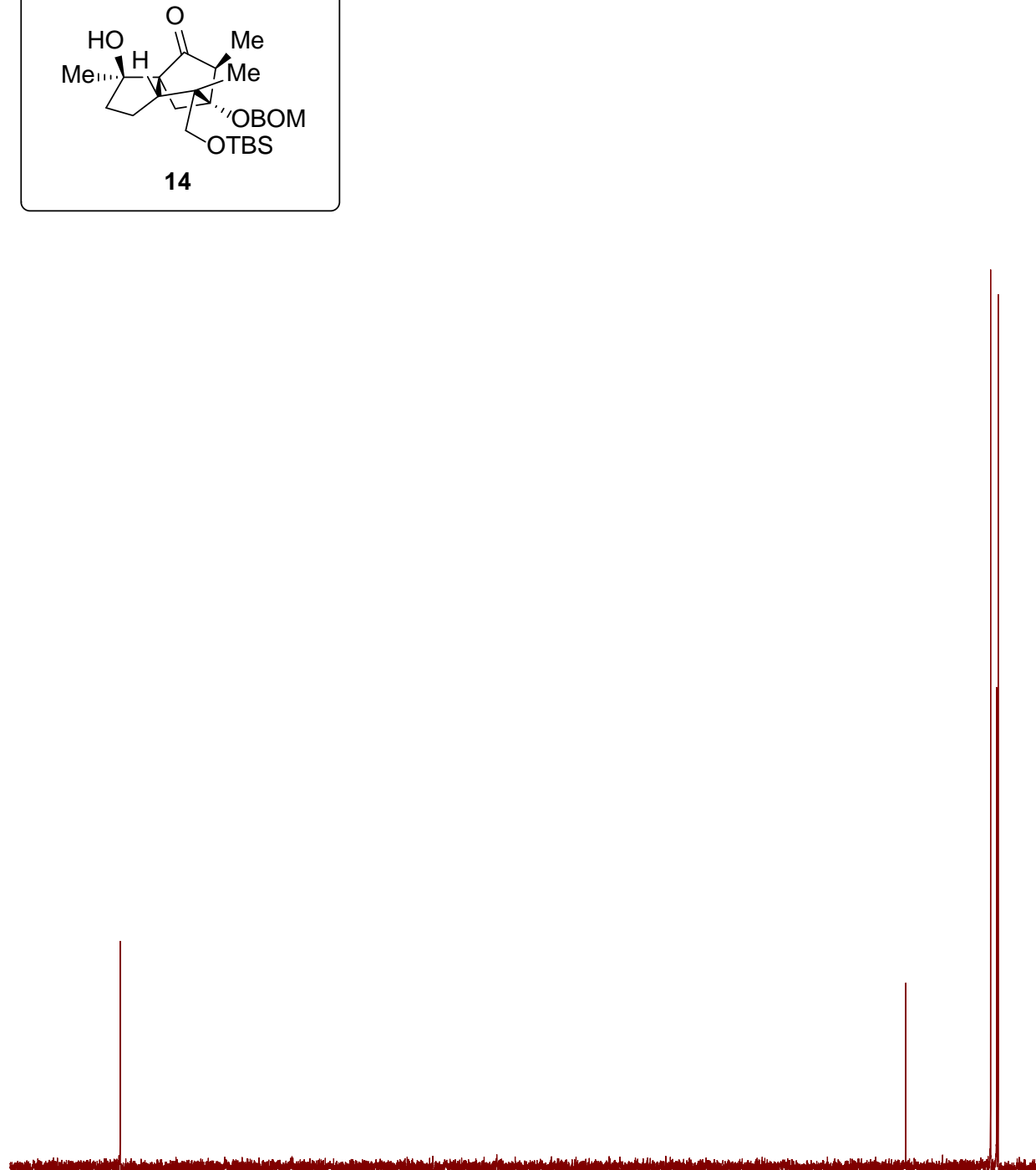


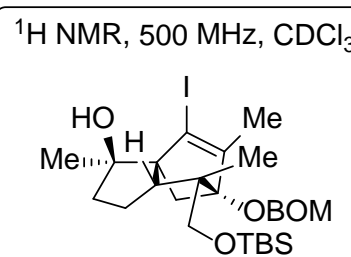

15

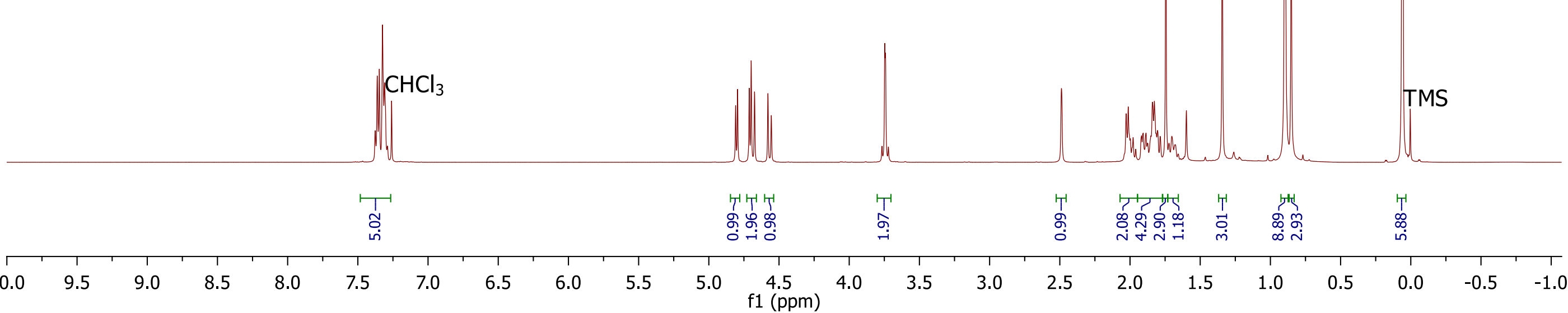




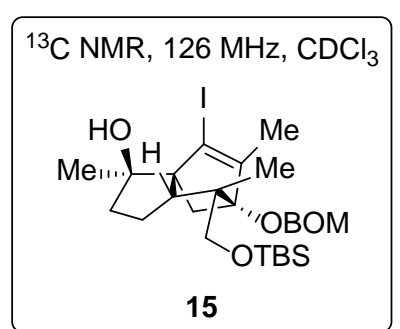

\begin{tabular}{|c|c|c|c|c|c|c|c|c|c|c|c|c|c|c|c|c|c|c|c|c|c|c|c|c|}
\hline 230 & 220 & 210 & 200 & 190 & 180 & 170 & 160 & 150 & 140 & 130 & 120 & $\begin{array}{c}110 \\
\mathrm{f} 1 \stackrel{(\mathrm{ppm})}{ }\end{array}$ & 100 & 90 & 80 & 70 & 60 & 50 & 40 & 30 & 20 & 10 & 0 & -10 \\
\hline
\end{tabular}



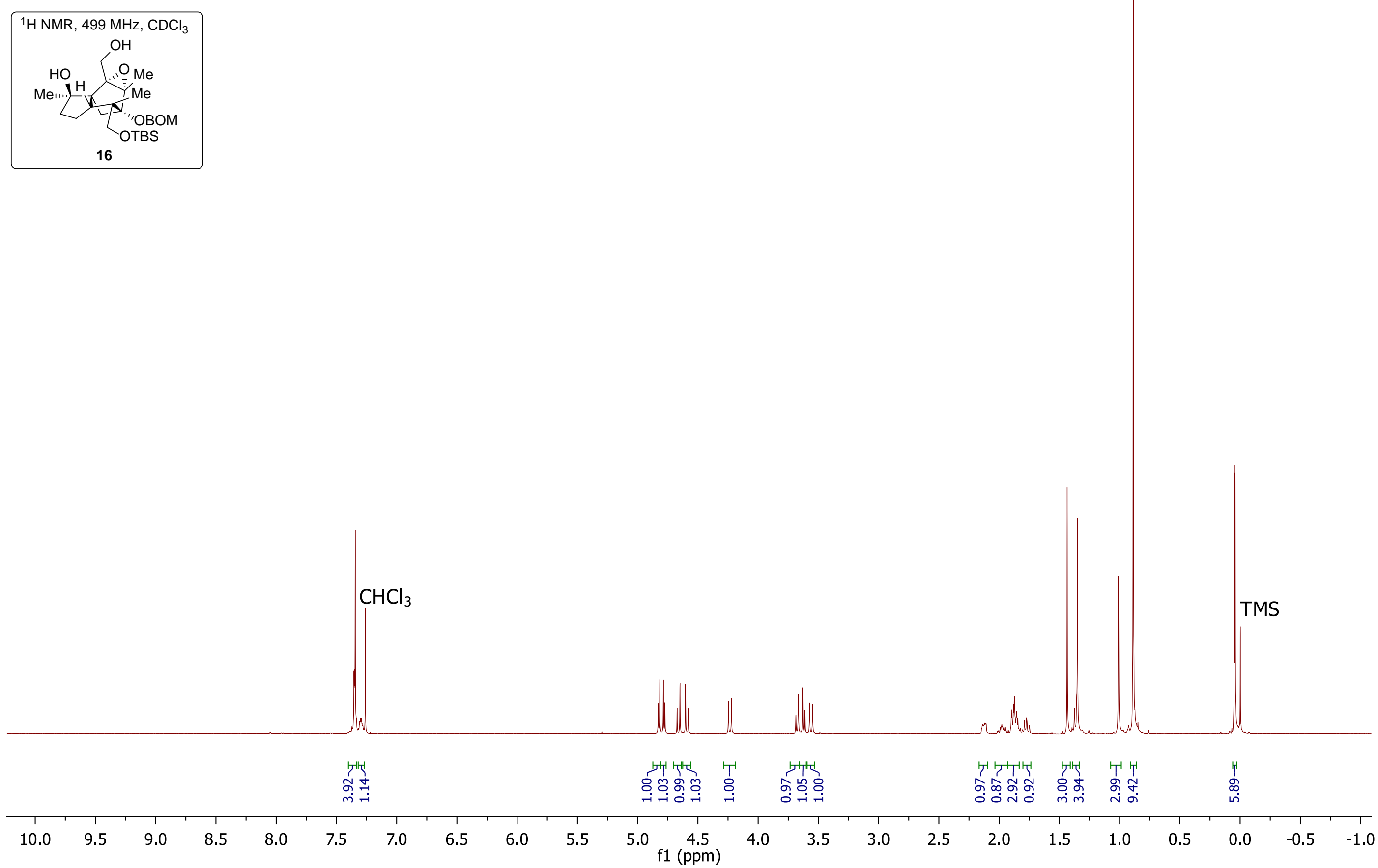

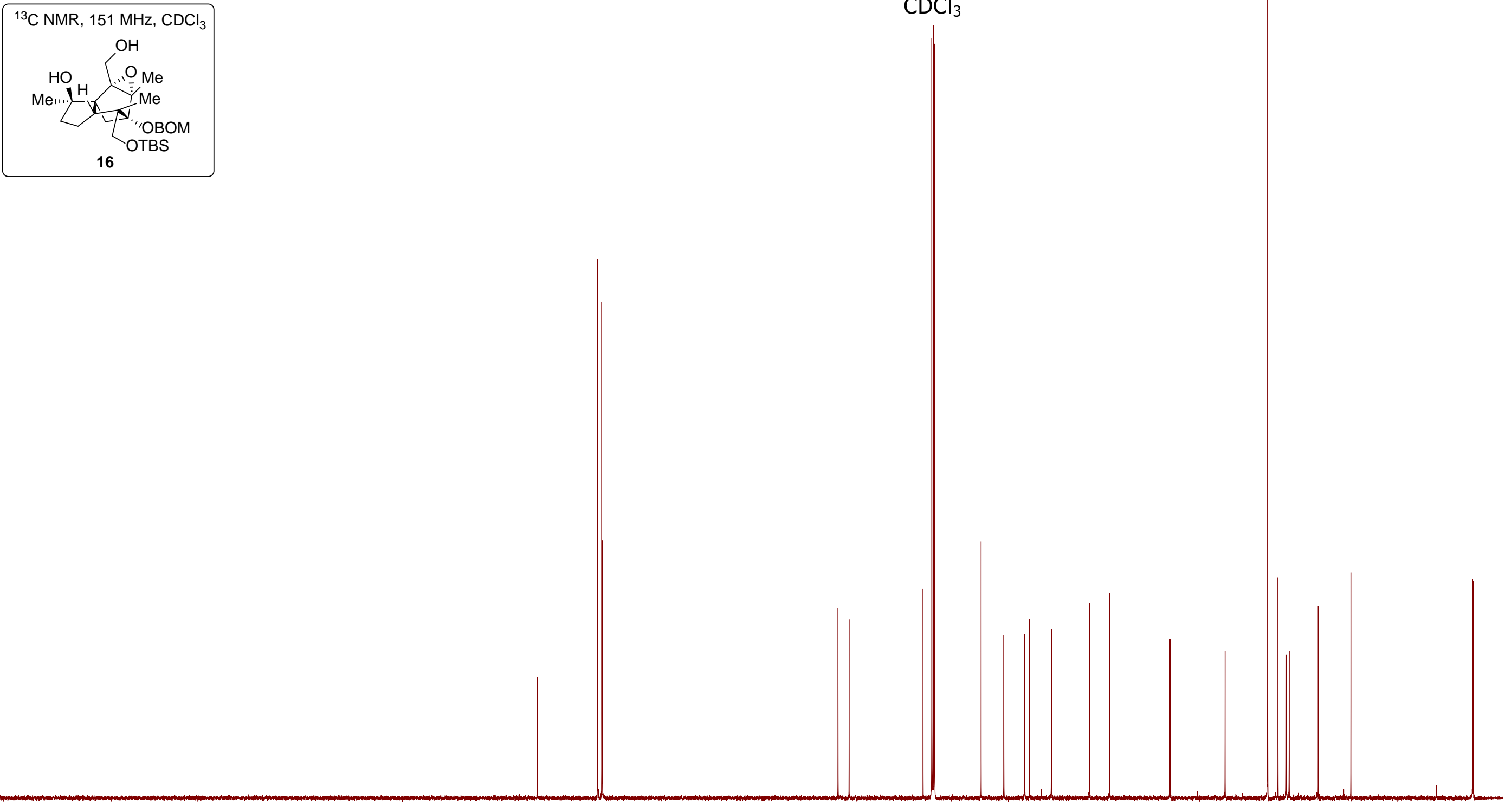

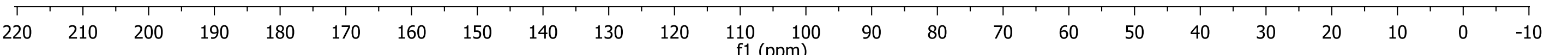




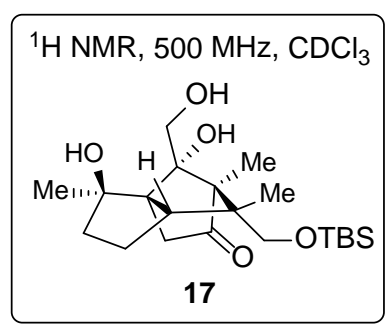

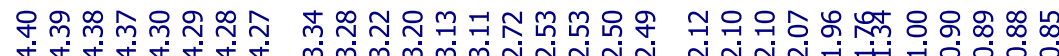

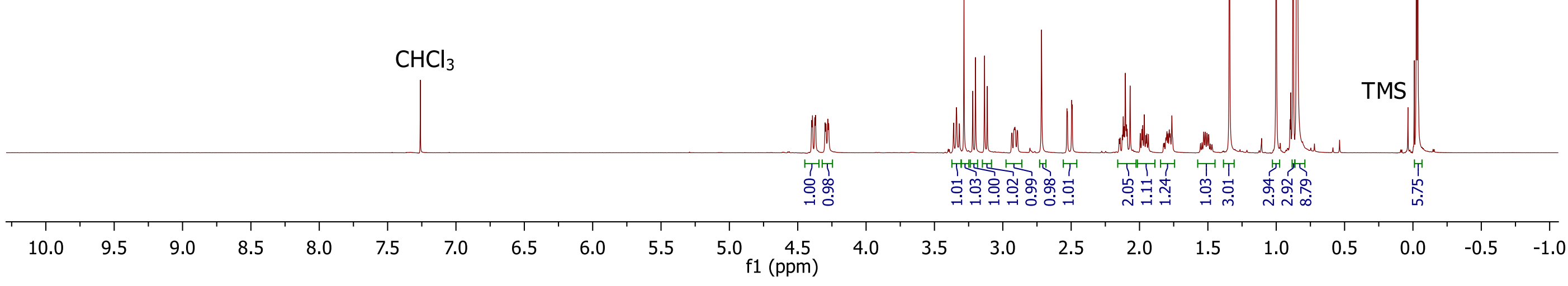



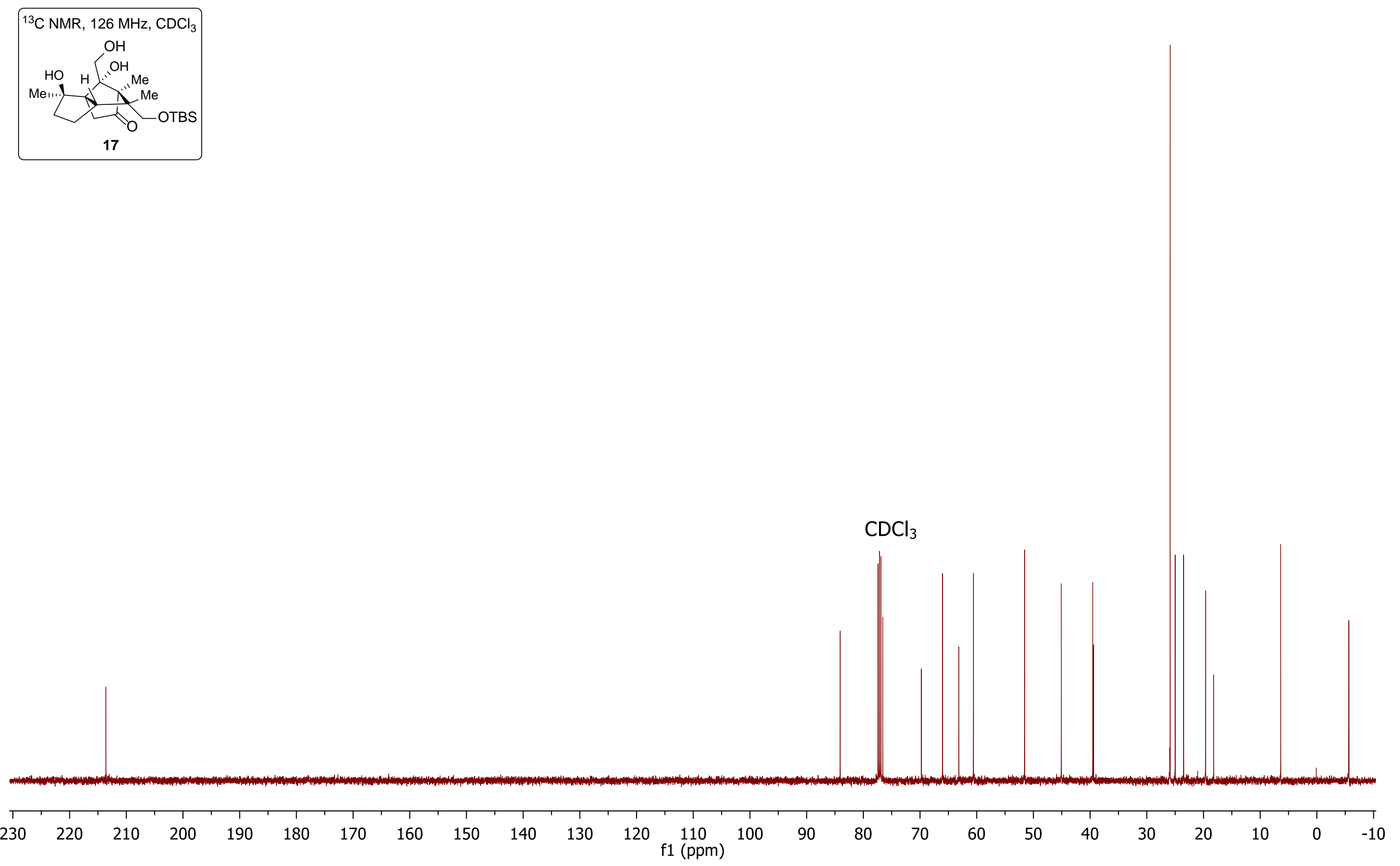

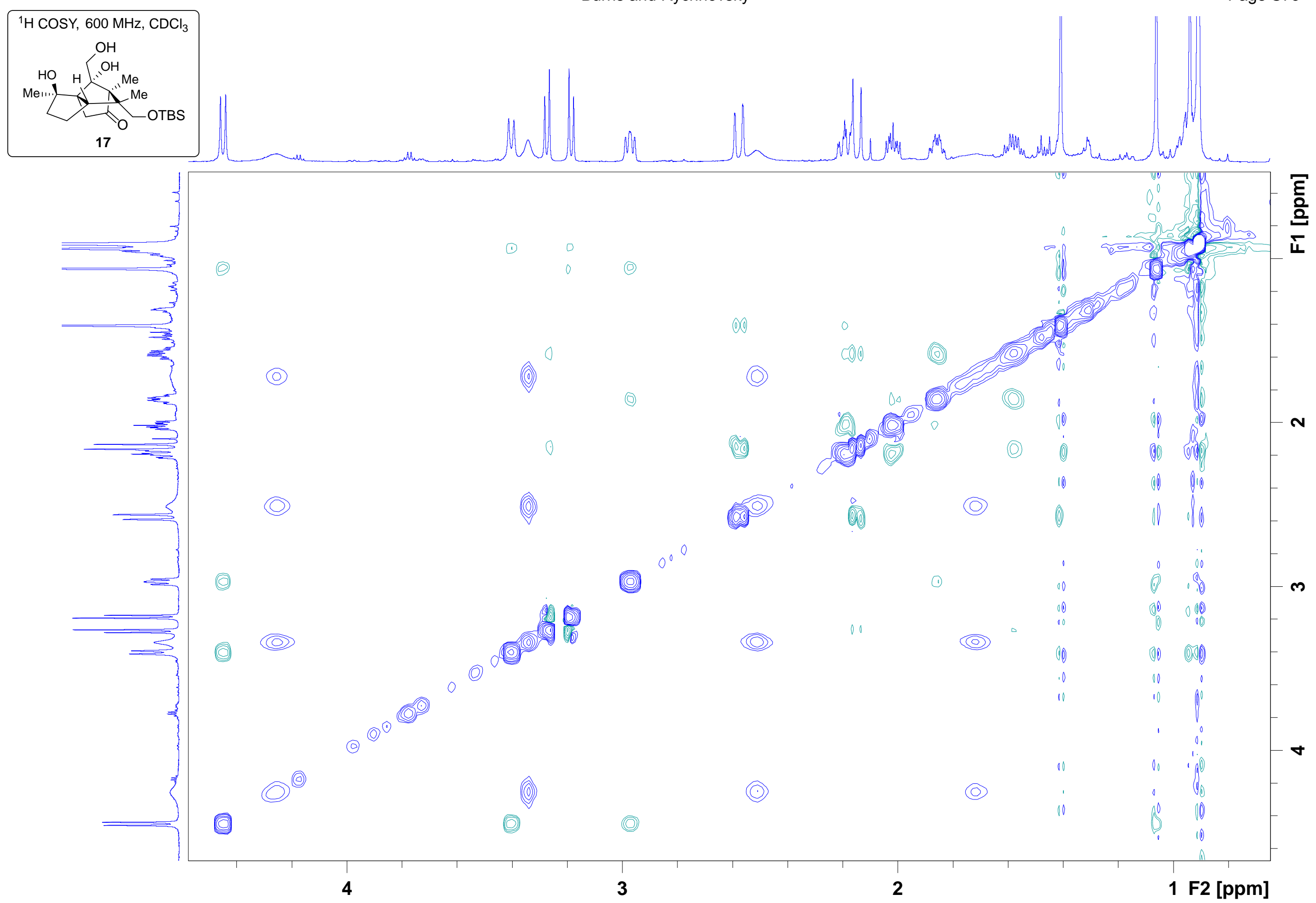


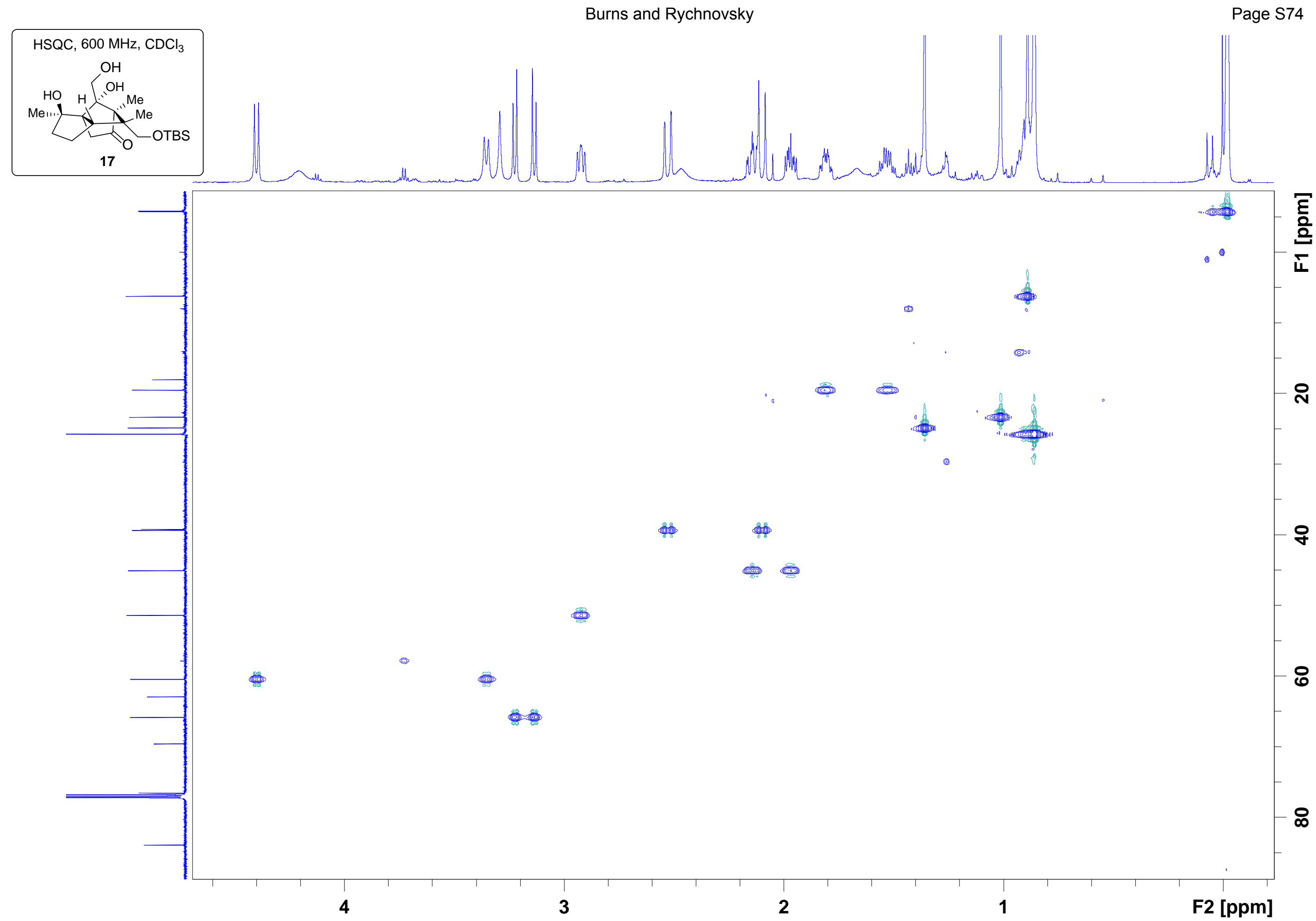



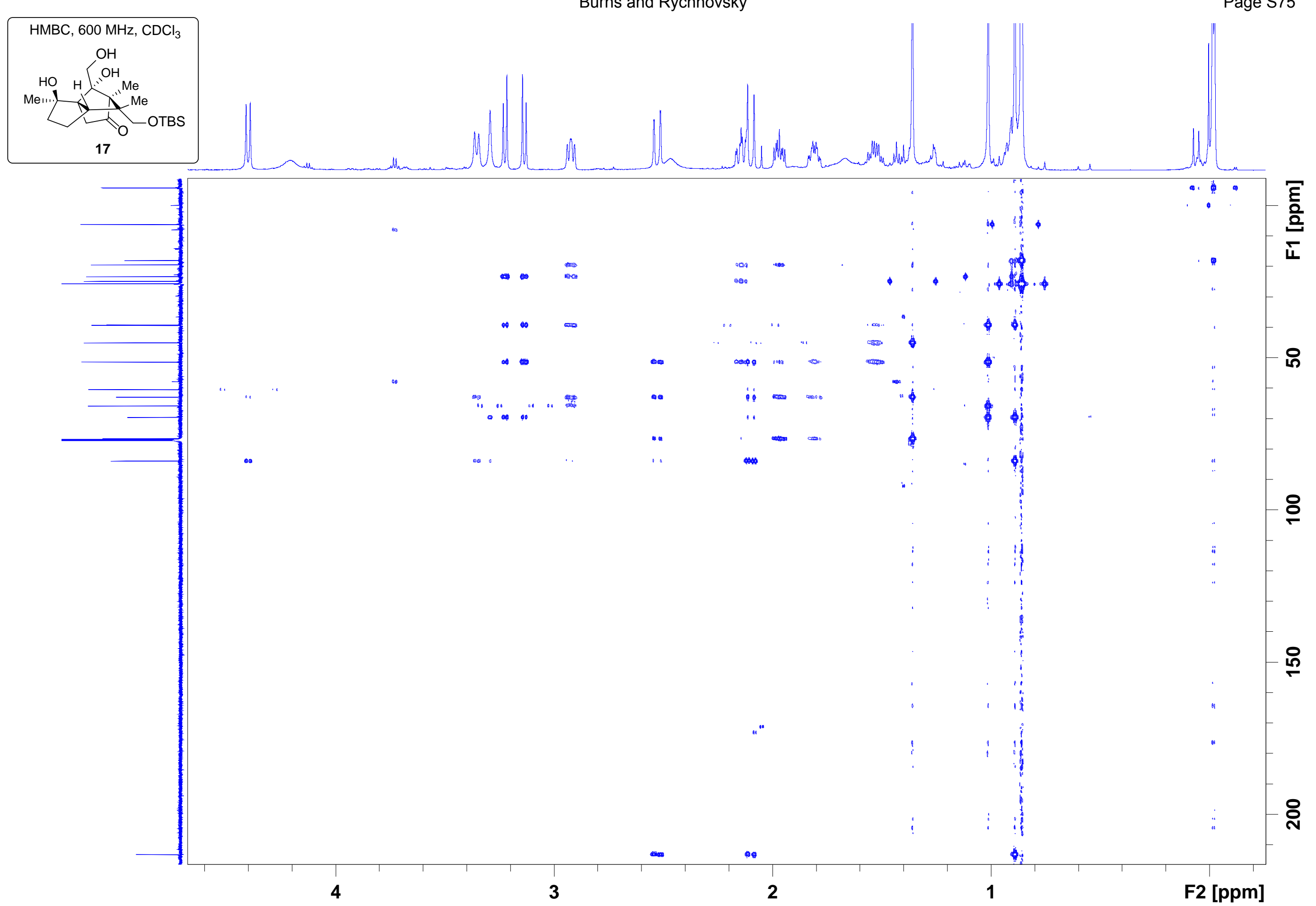

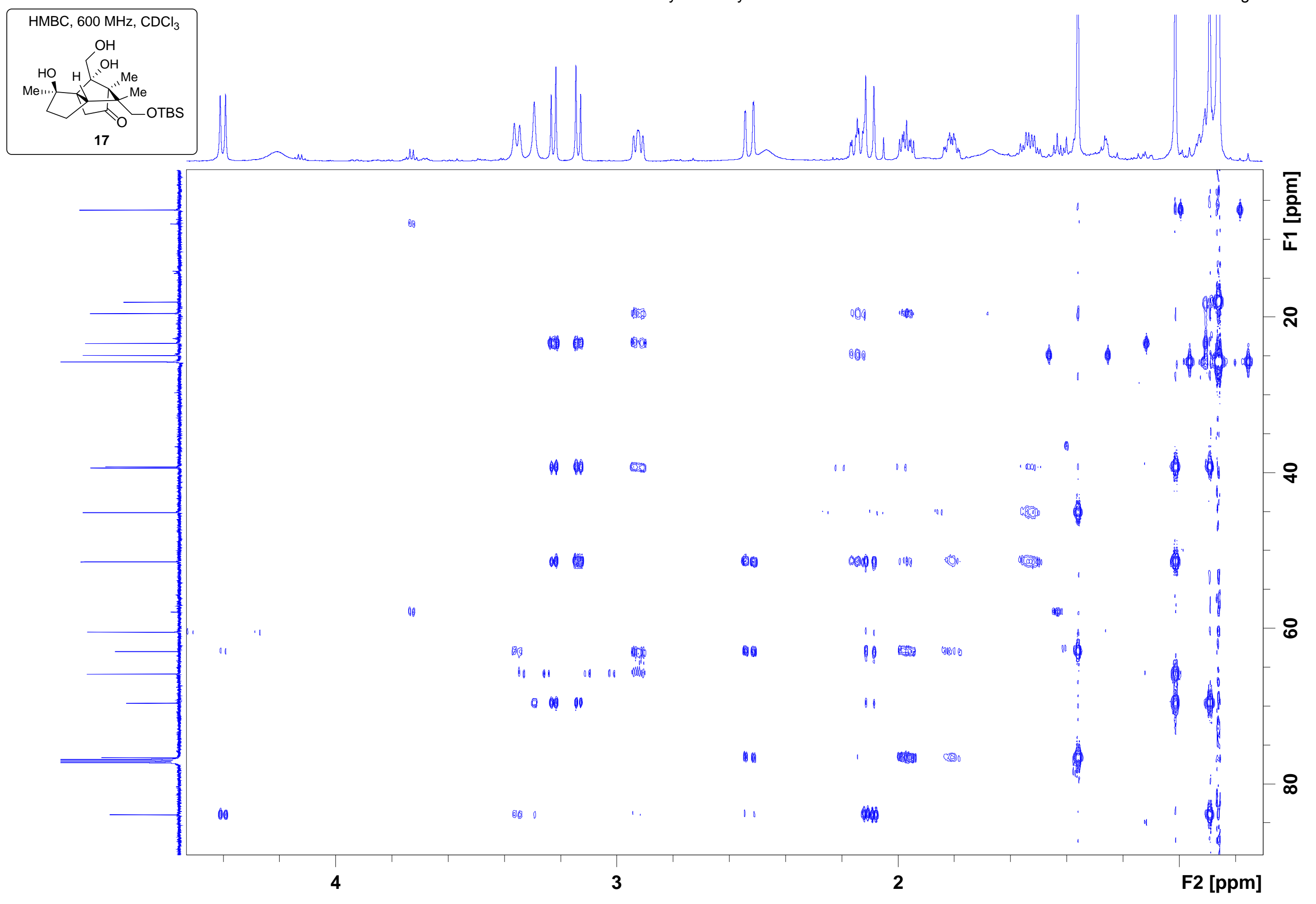

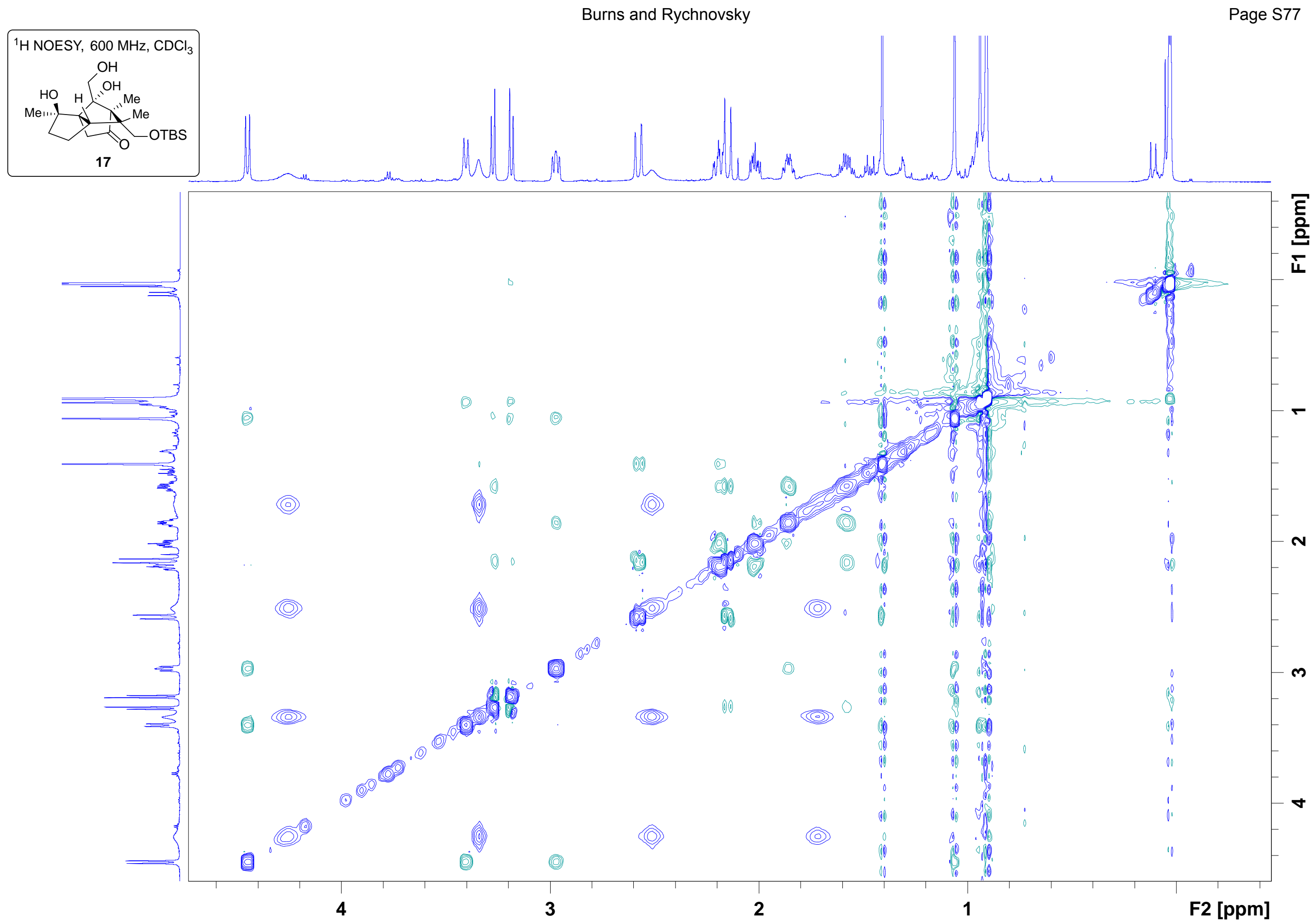

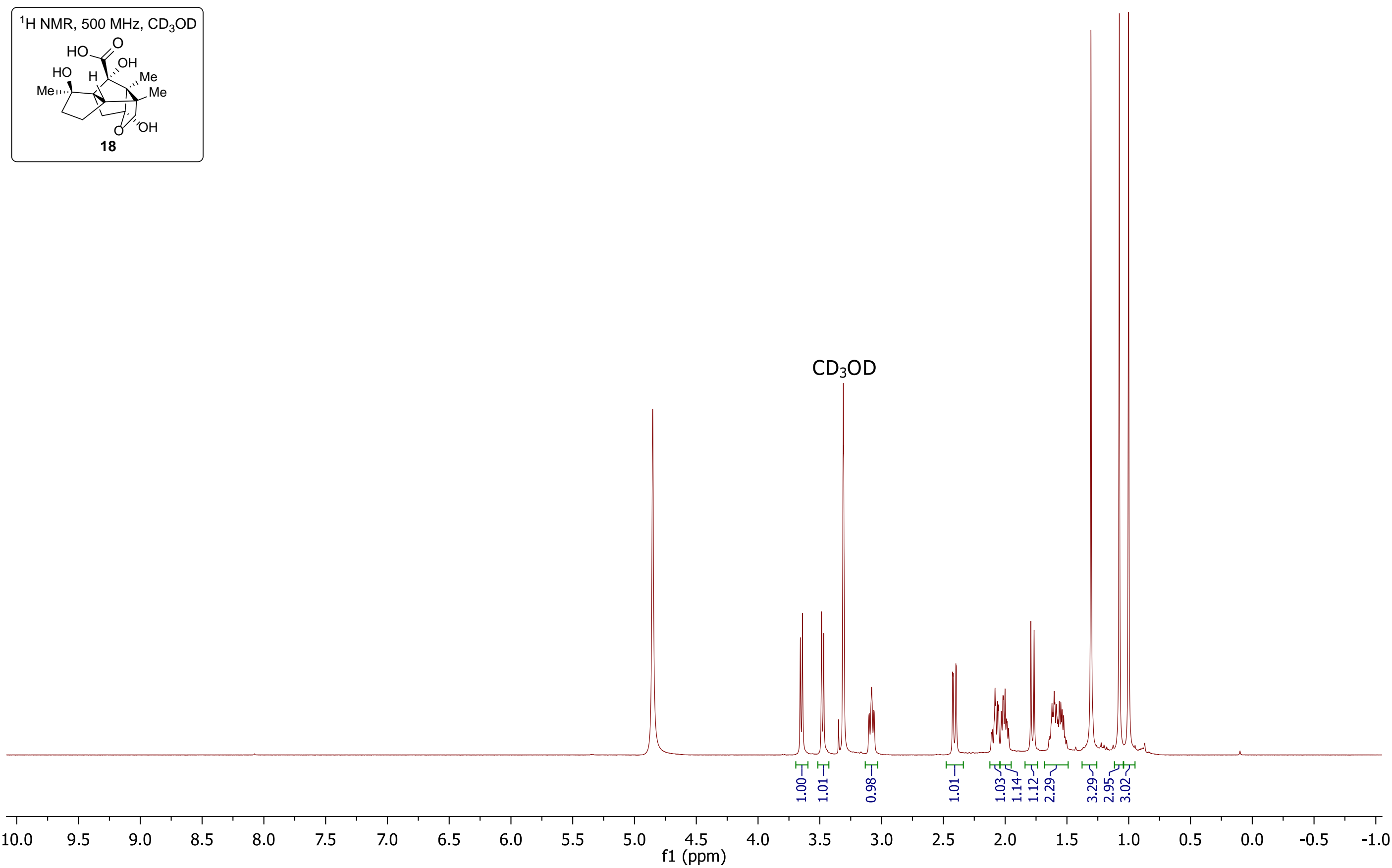

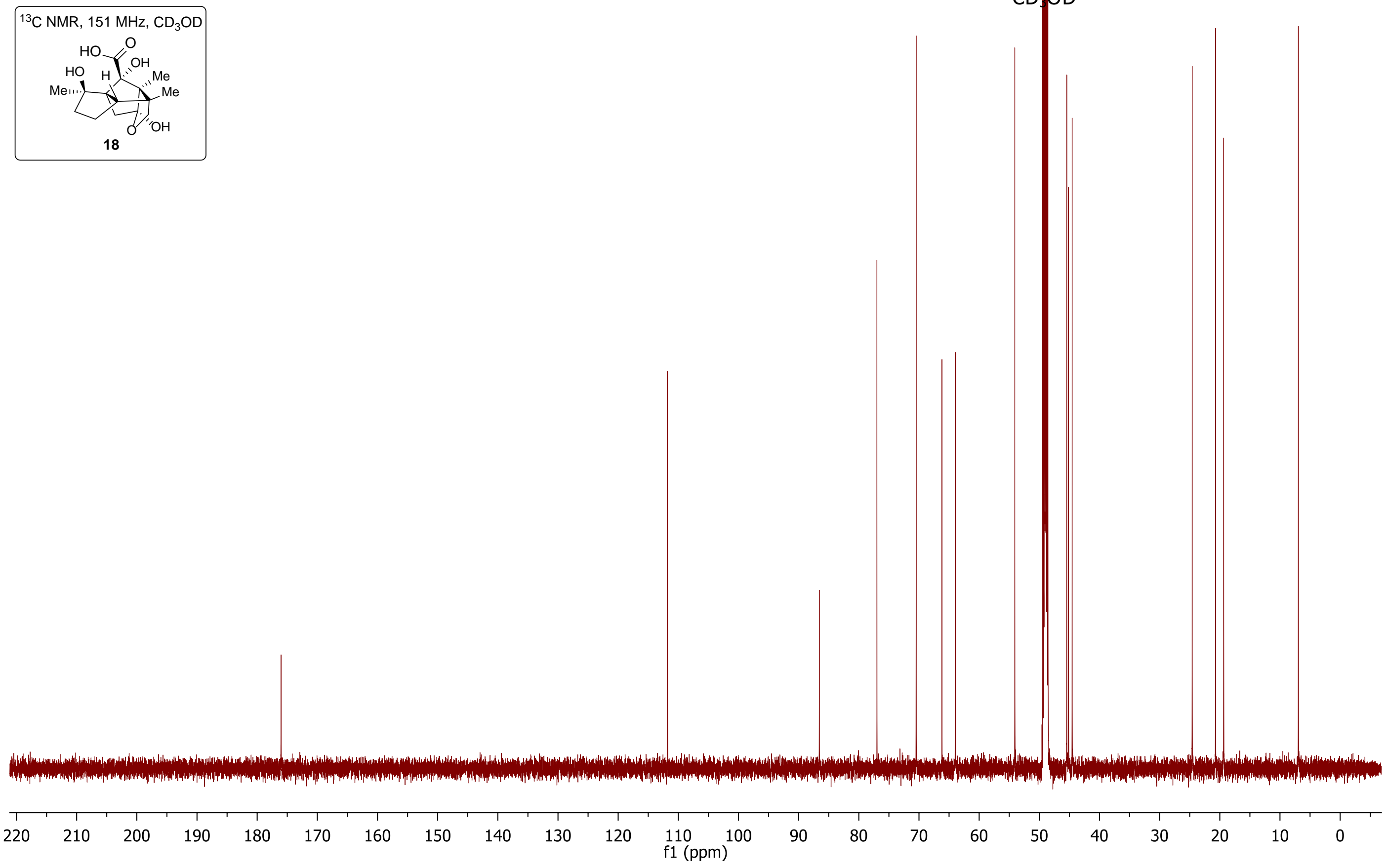
${ }^{1} \mathrm{H} \mathrm{NMR}, 500 \mathrm{MHz}, \mathrm{CDCl}_{3}$

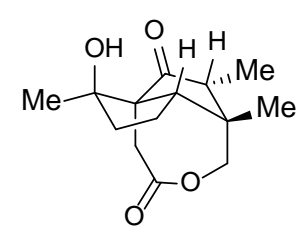

21

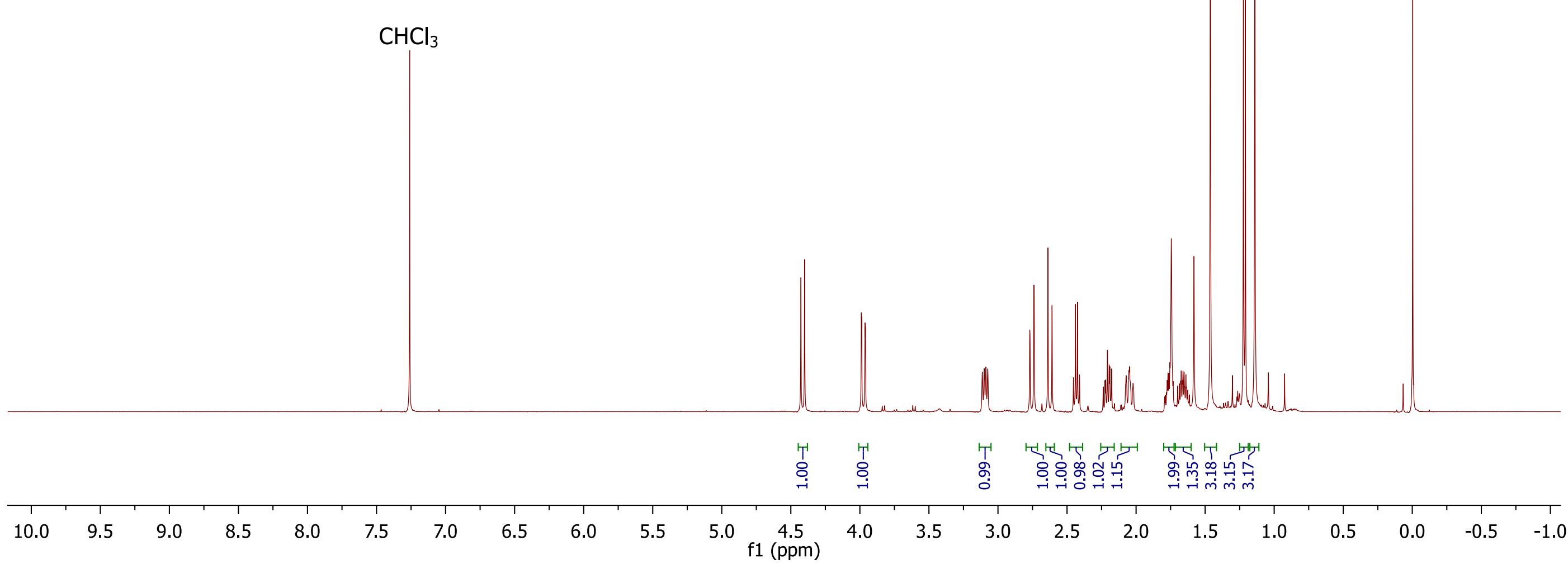




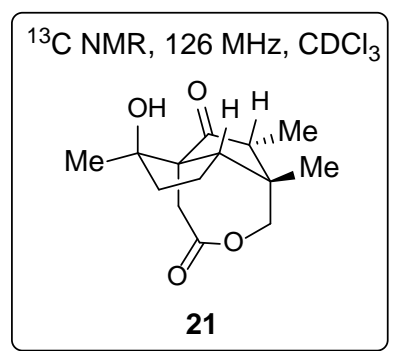

\section{$\mathrm{CDCl}_{3}$}

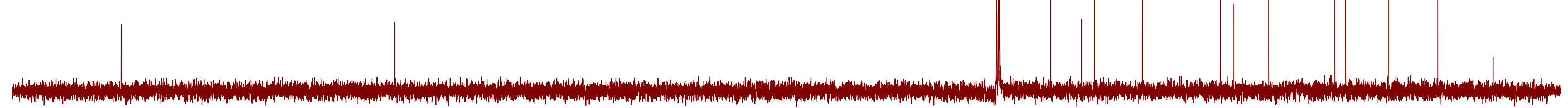

\begin{tabular}{|c|c|c|c|c|c|c|c|c|c|c|c|c|c|c|c|c|c|c|c|c|c|c|c|}
\hline יו & 1 & 1 & 10 & 1 & 1 & 16 & 1 & 1 & 13 & & 11 & 1 & 1 & To & 1 & 1 & 1 & 1 & 1 & 1 & 1 & 1 & -10 \\
\hline $230 \quad 220$ & 210 & 200 & 190 & 180 & 170 & 160 & 150 & 140 & 130 & 120 & $\begin{array}{c}110 \\
\mathrm{f} 1 \\
(\mathrm{ppm})\end{array}$ & 100 & 90 & 80 & 70 & 60 & 50 & 40 & 30 & 20 & 10 & 0 & -10 \\
\hline
\end{tabular}




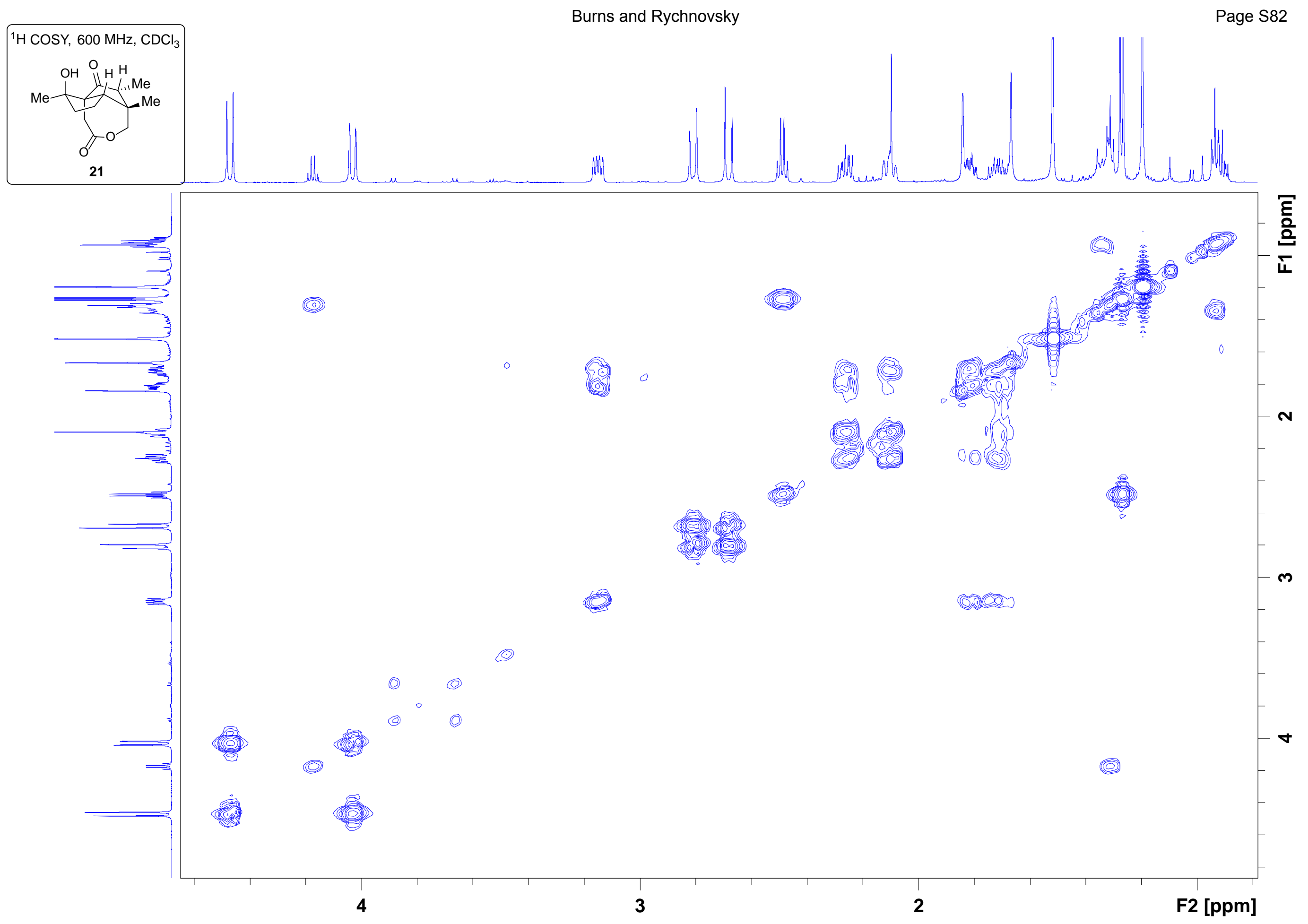




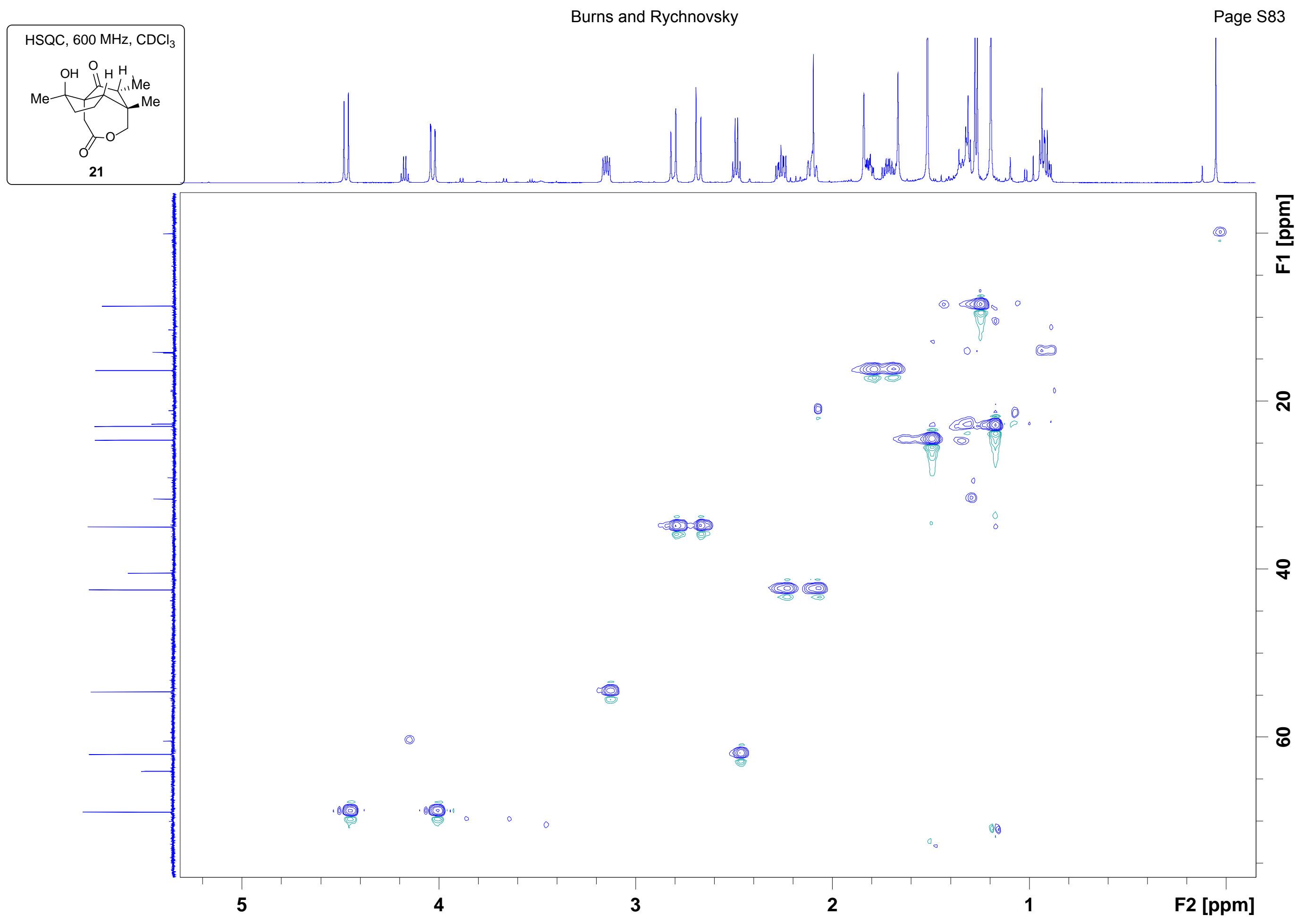




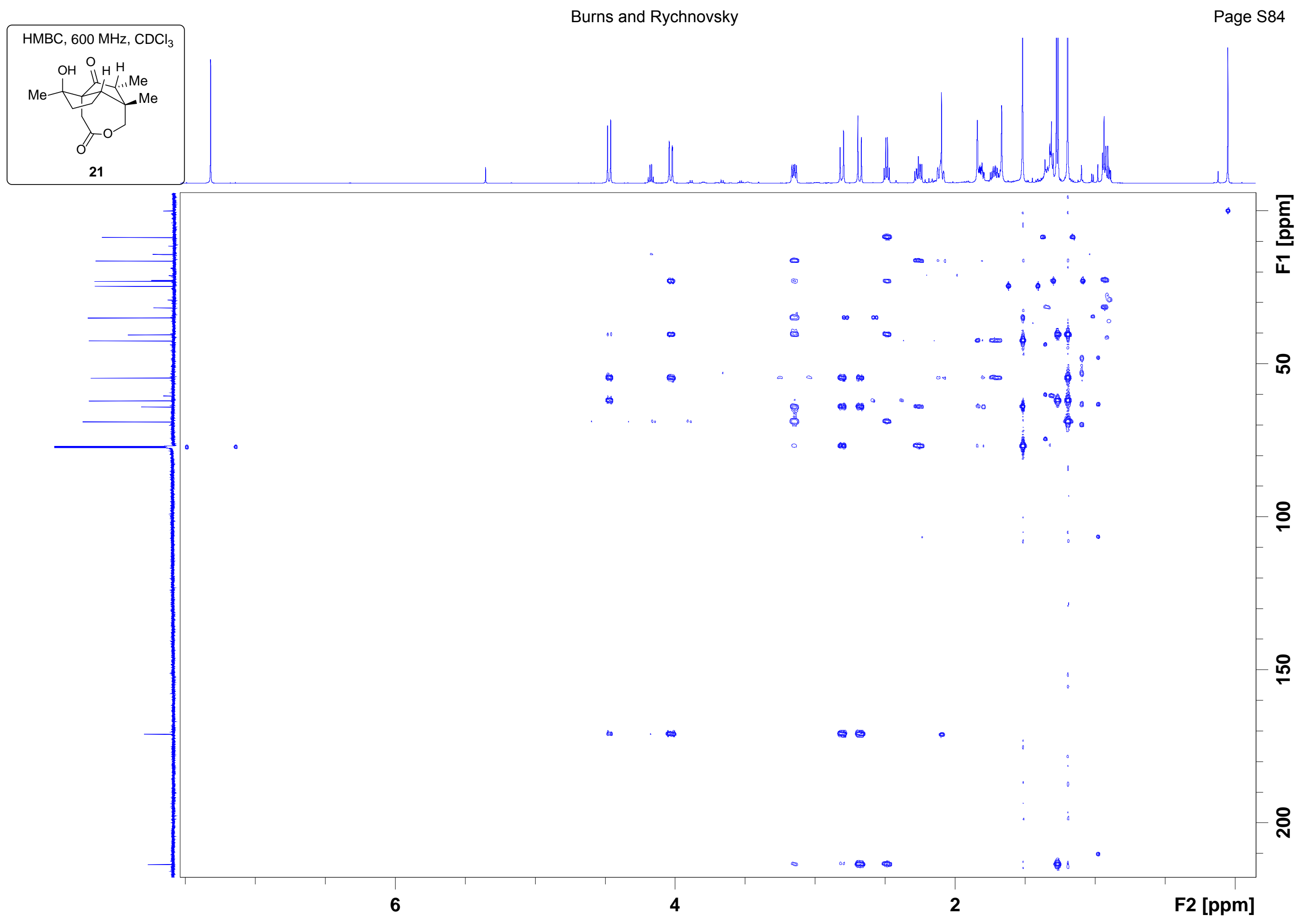




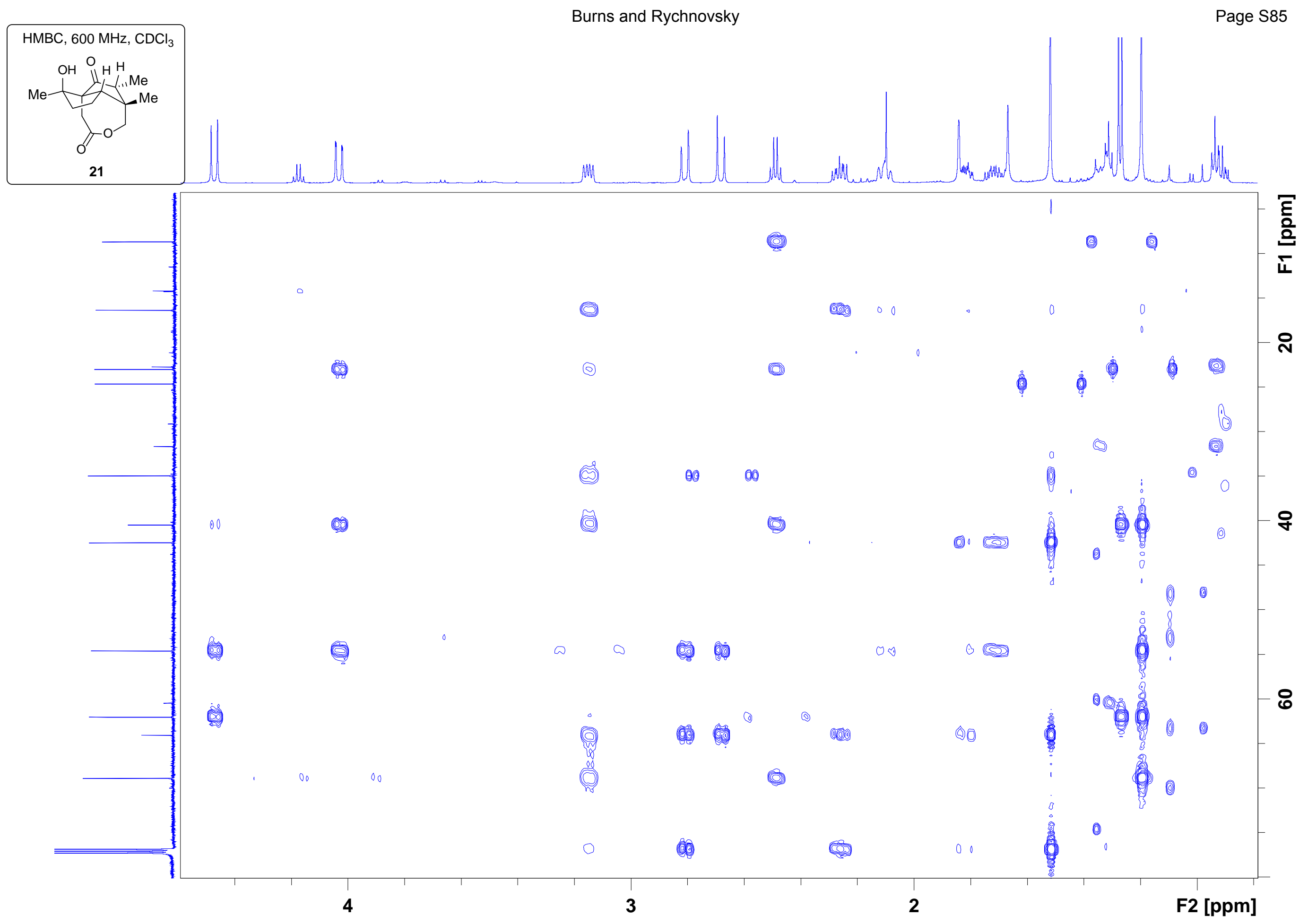




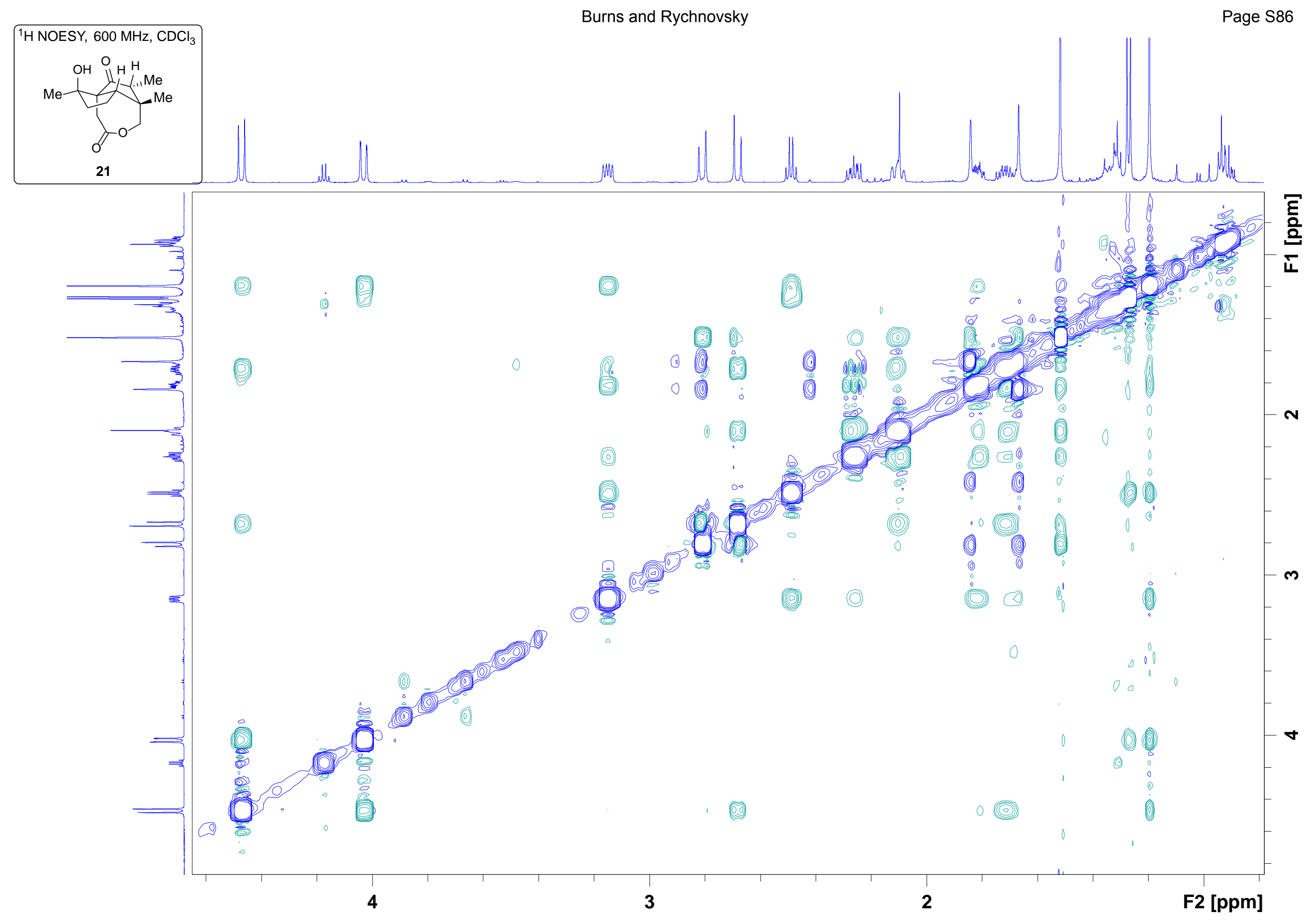




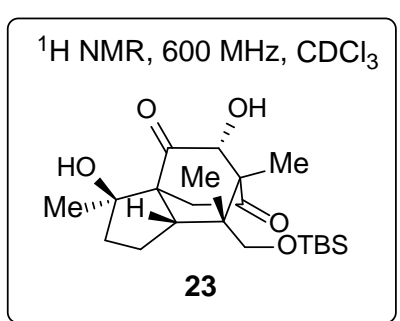

$\underbrace{\infty}$

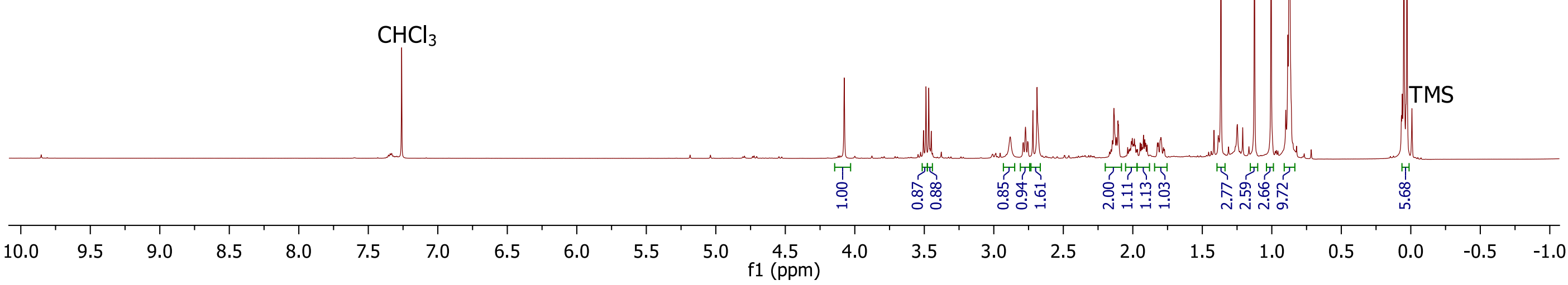




\section{$\mathrm{CDCl}_{3}$}

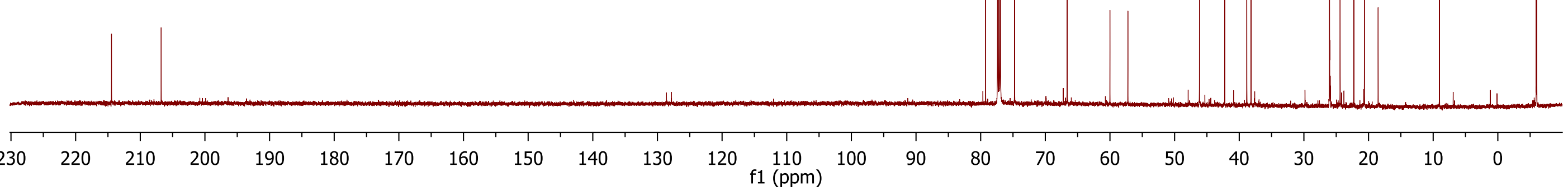




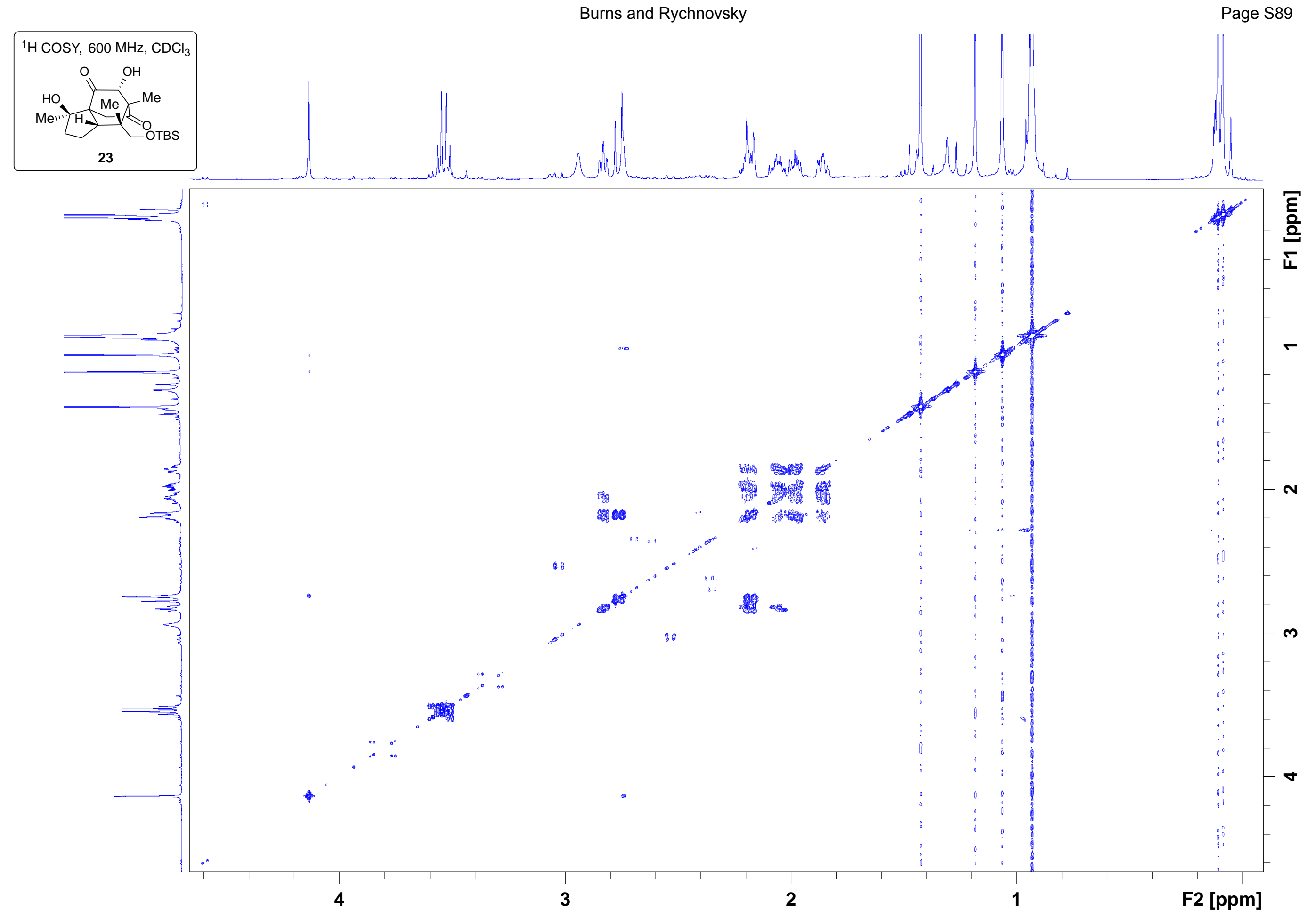



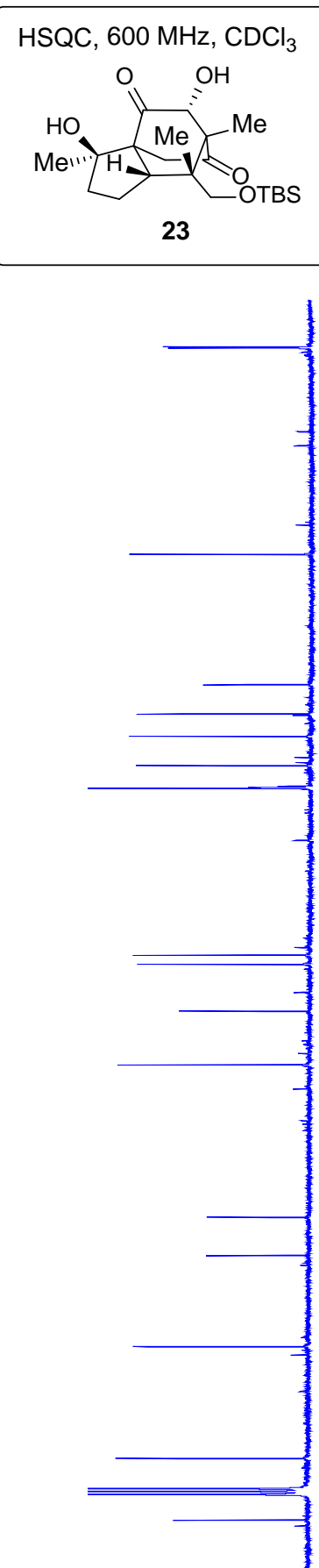
Nan

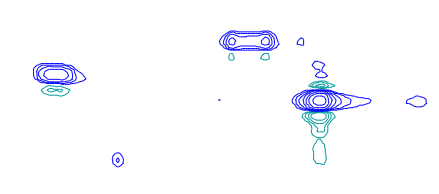




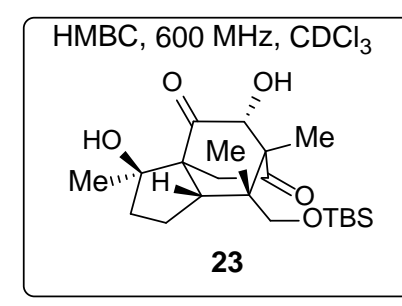

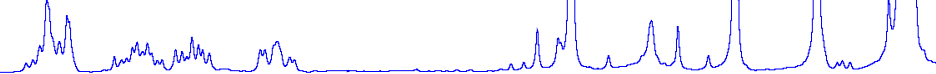

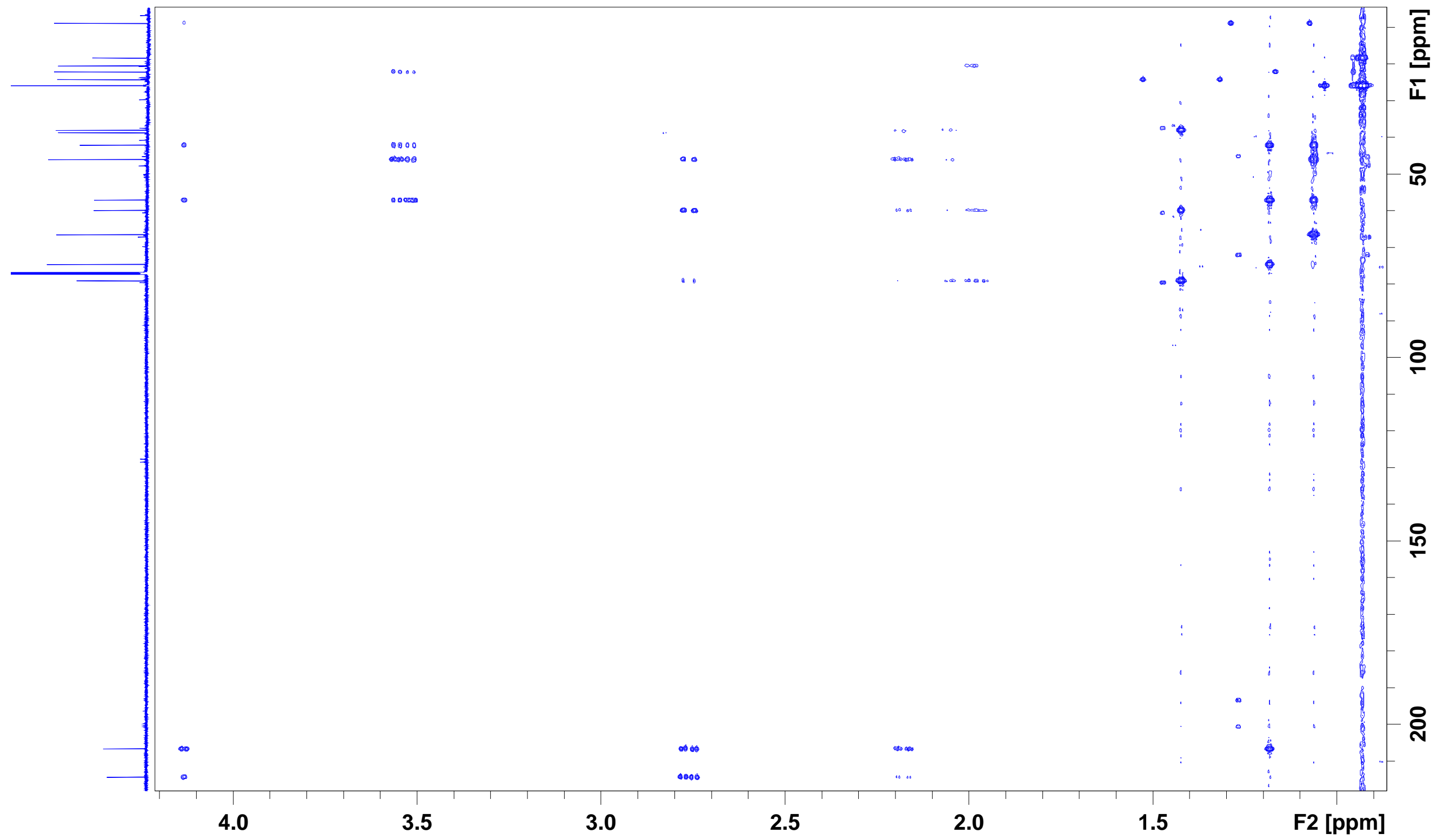



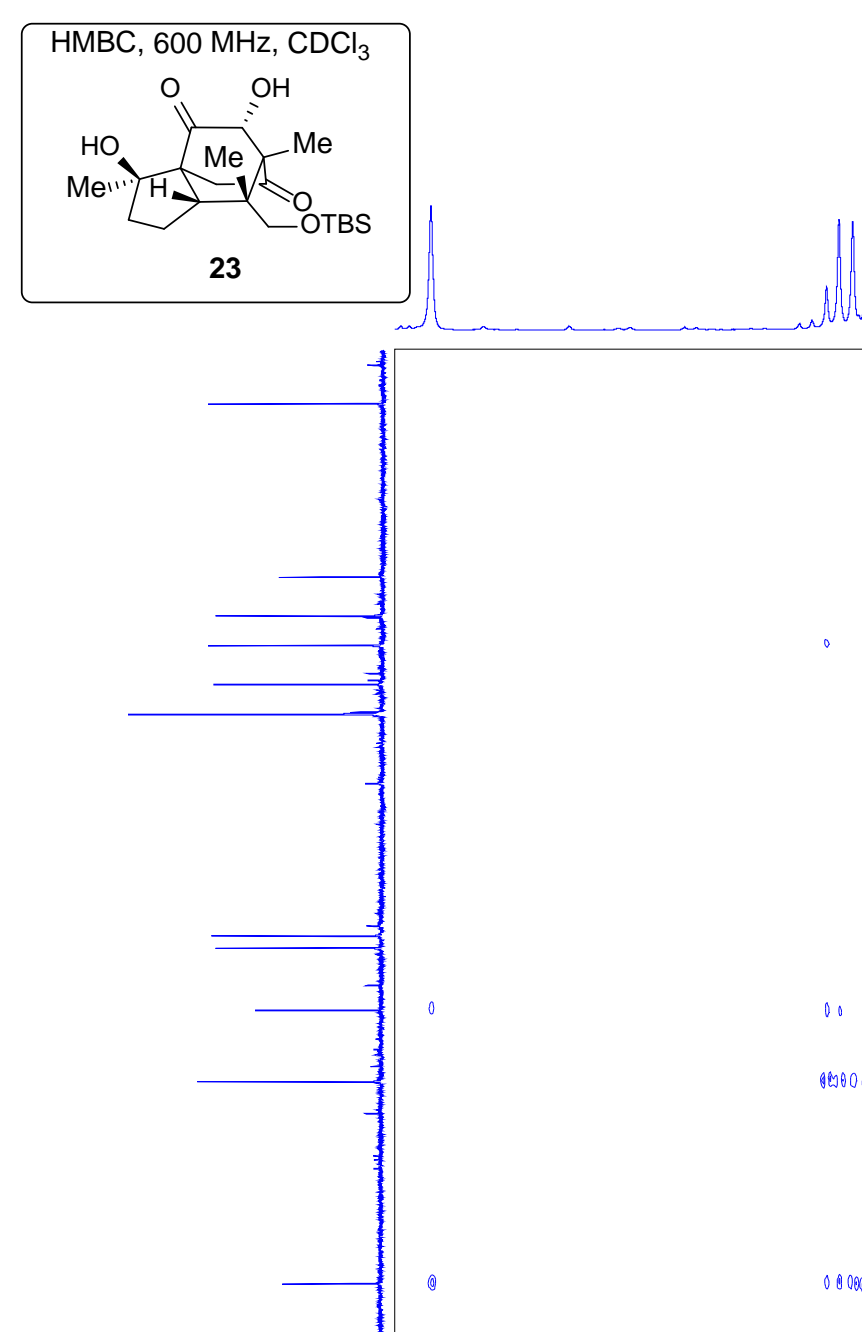

1000919 

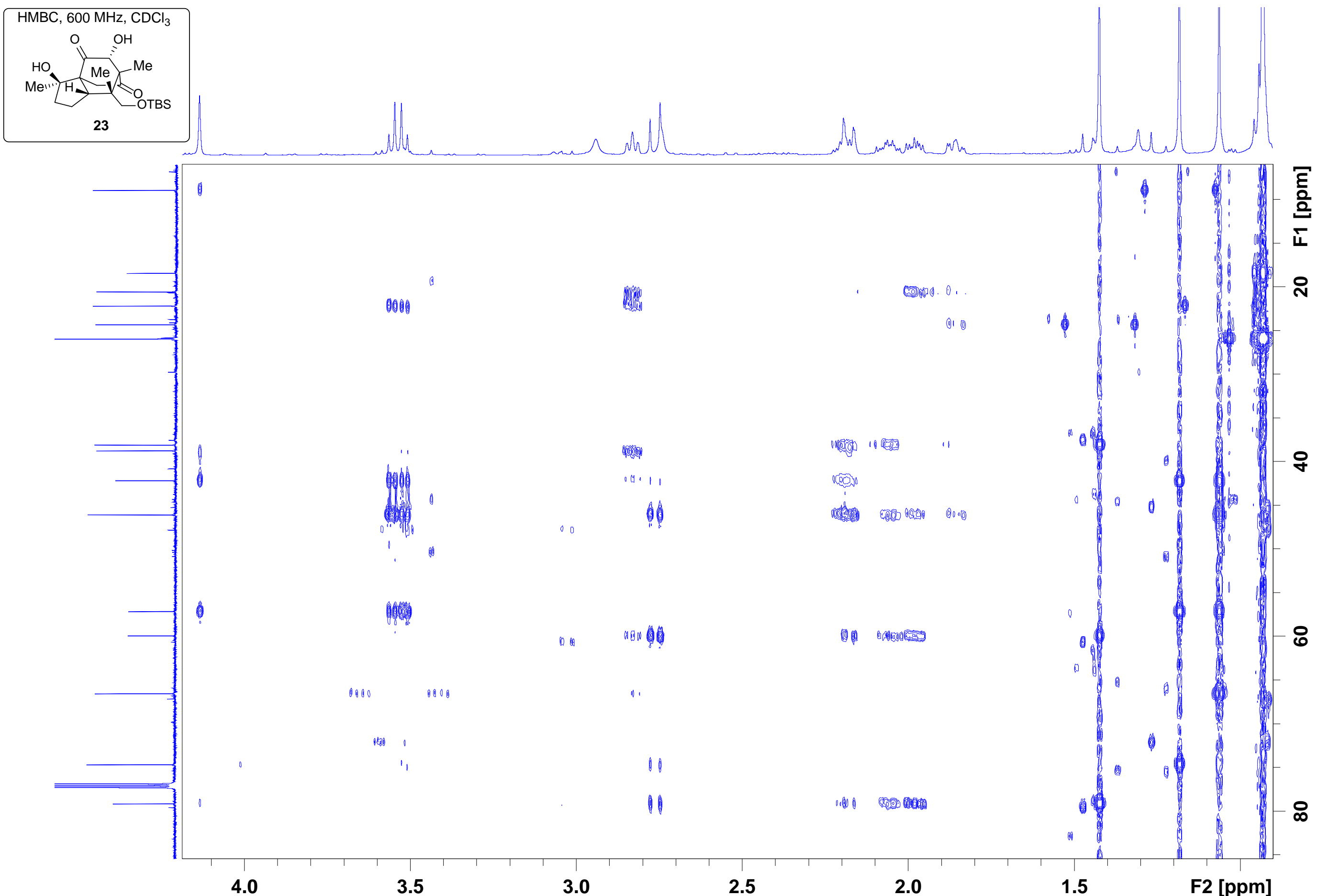

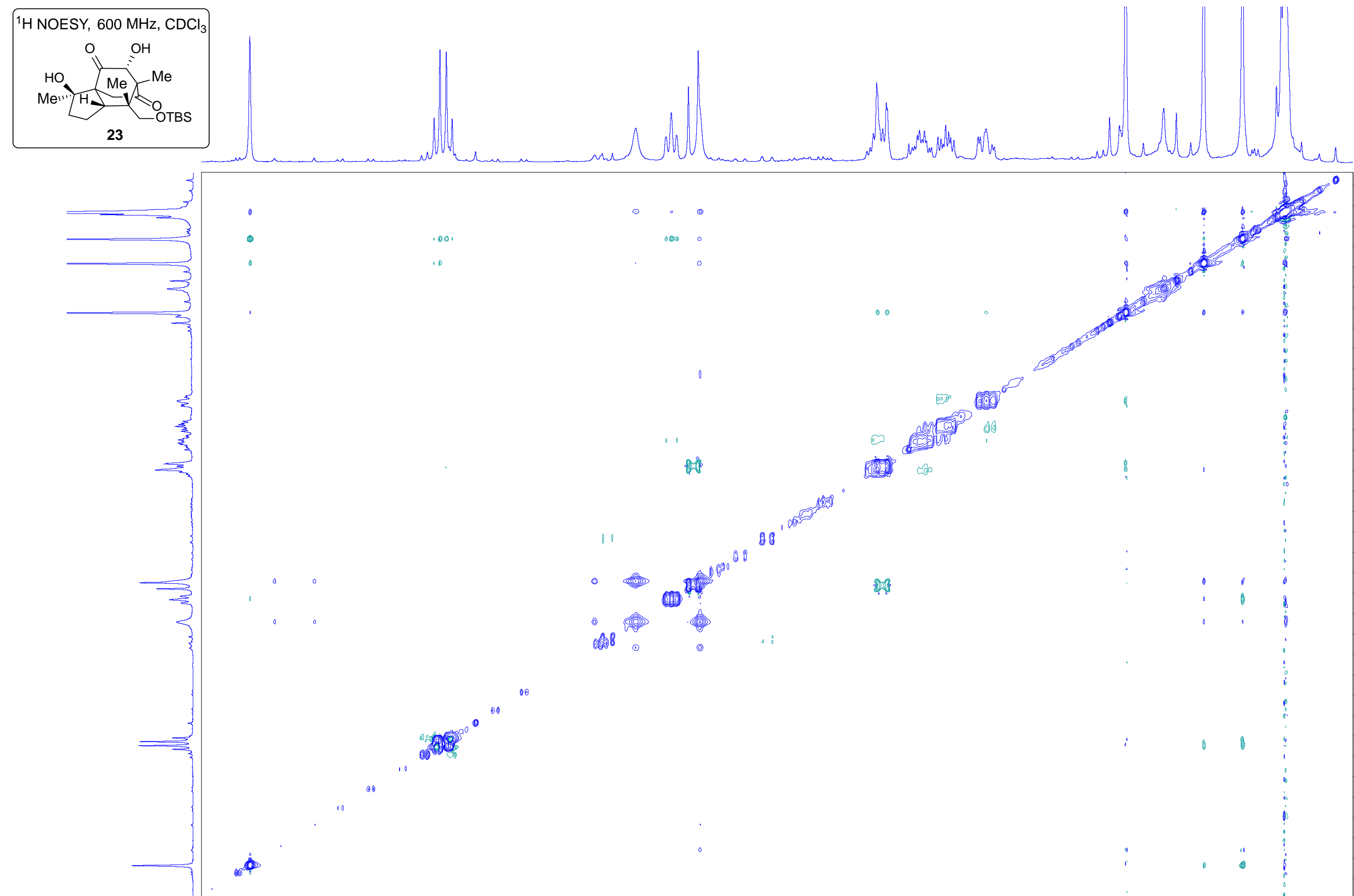

躇

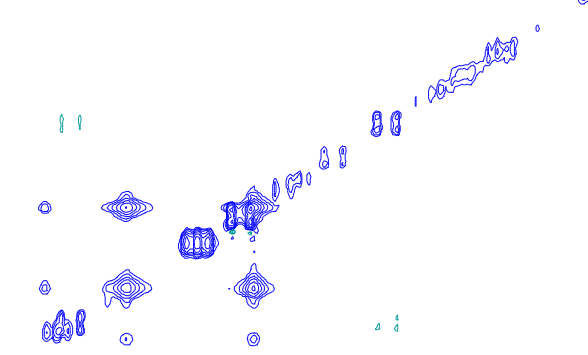




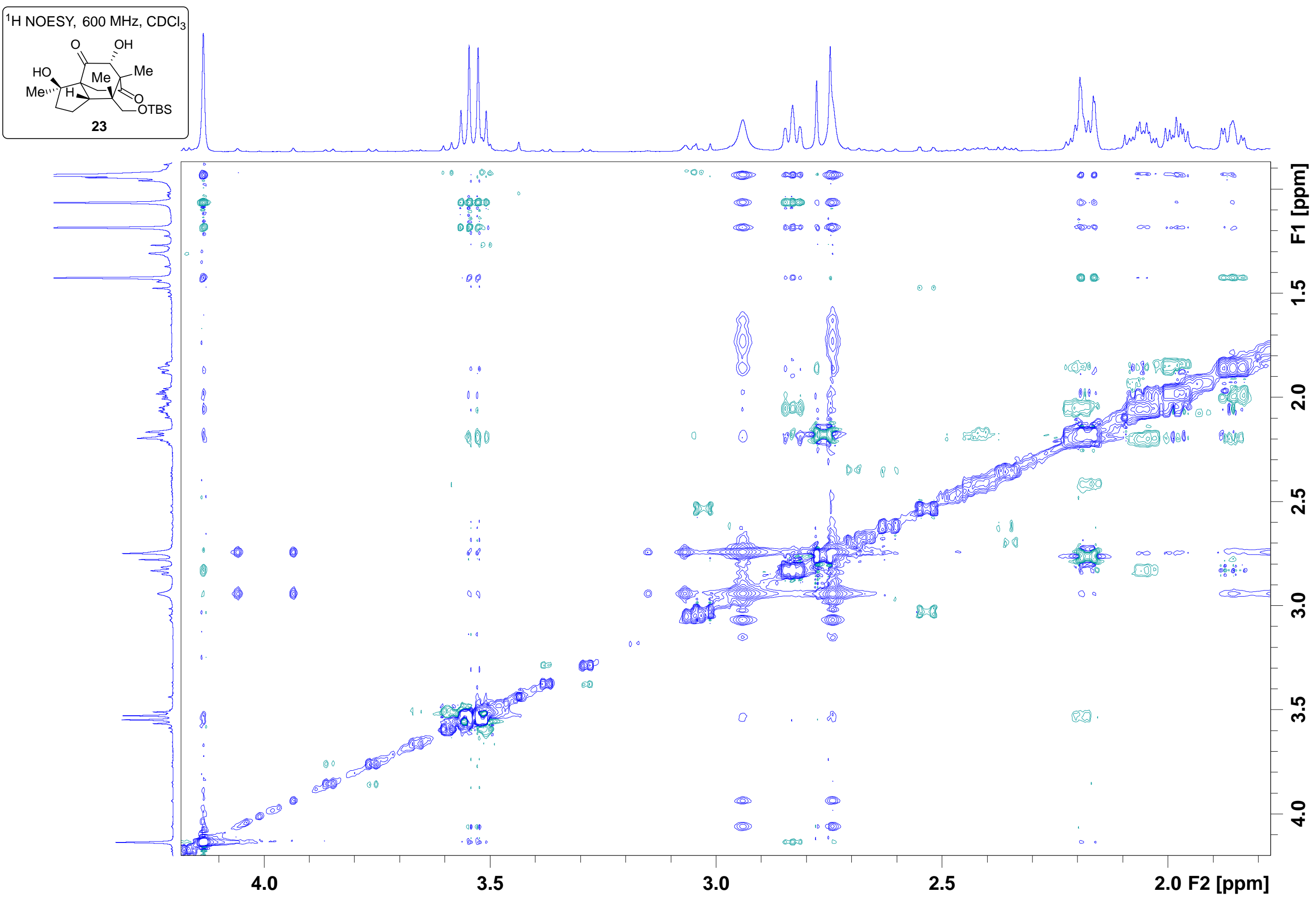




(士)-24

$( \pm)-24$

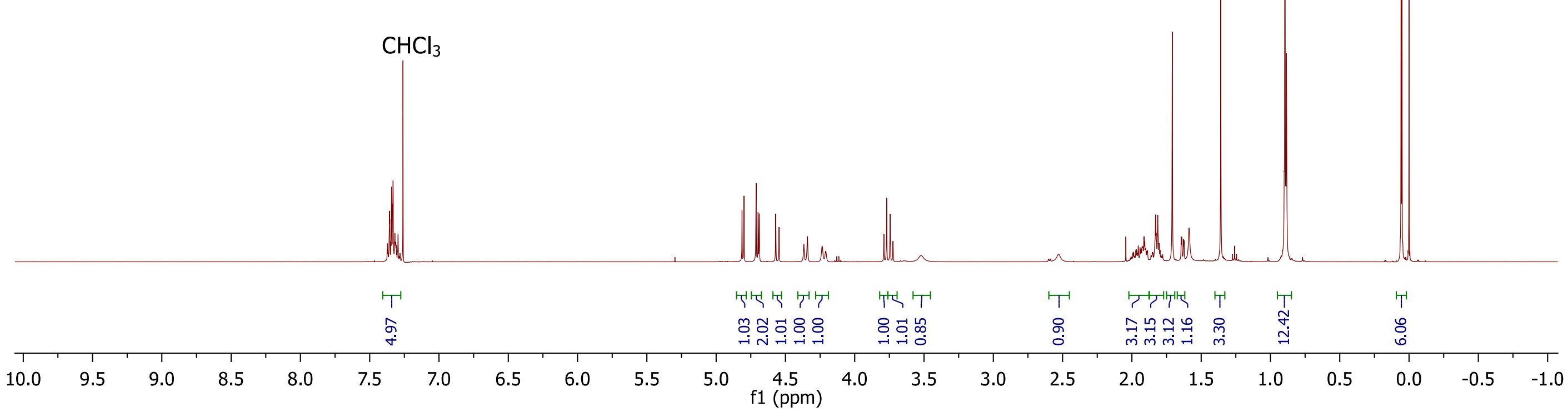



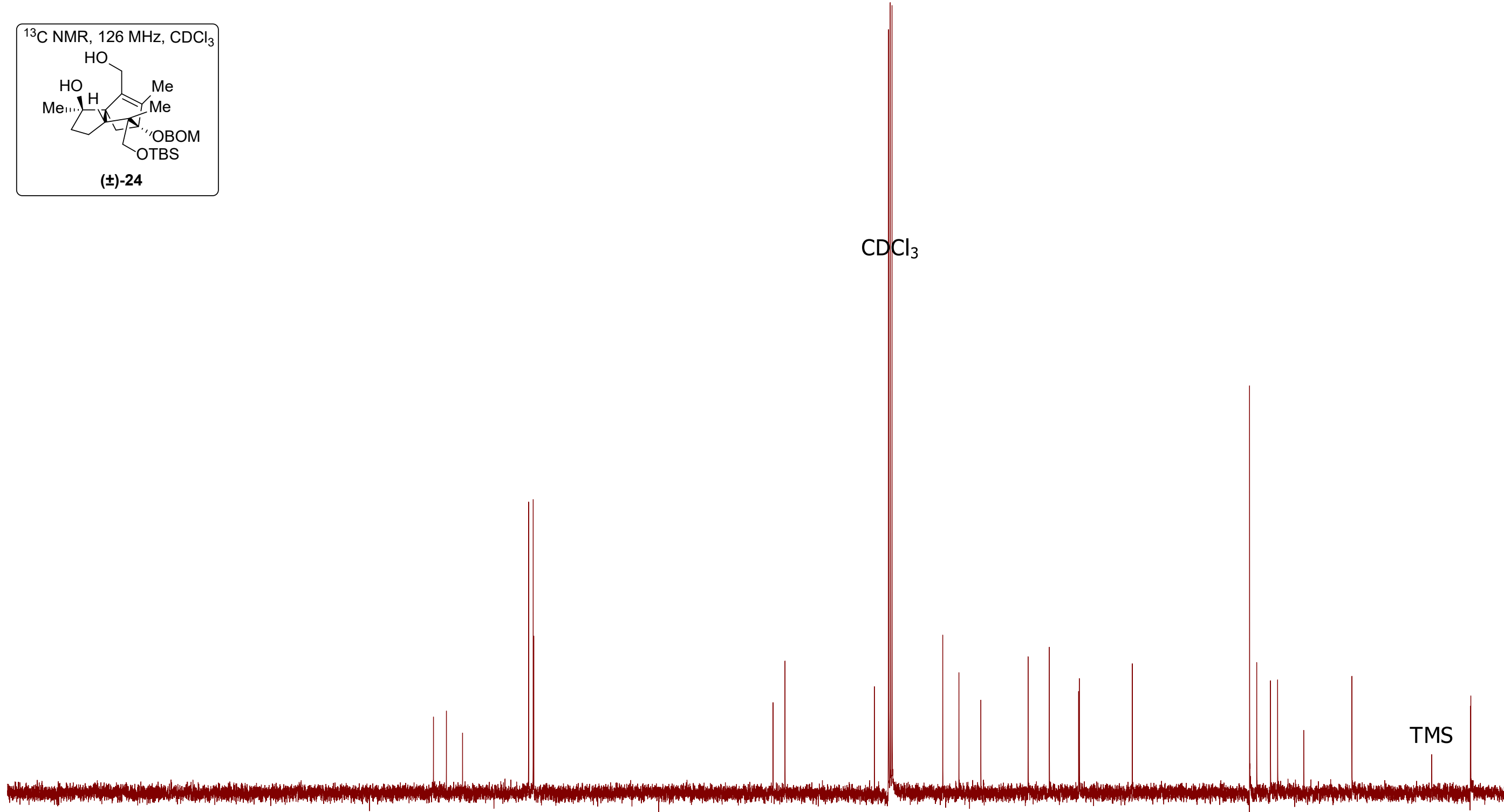

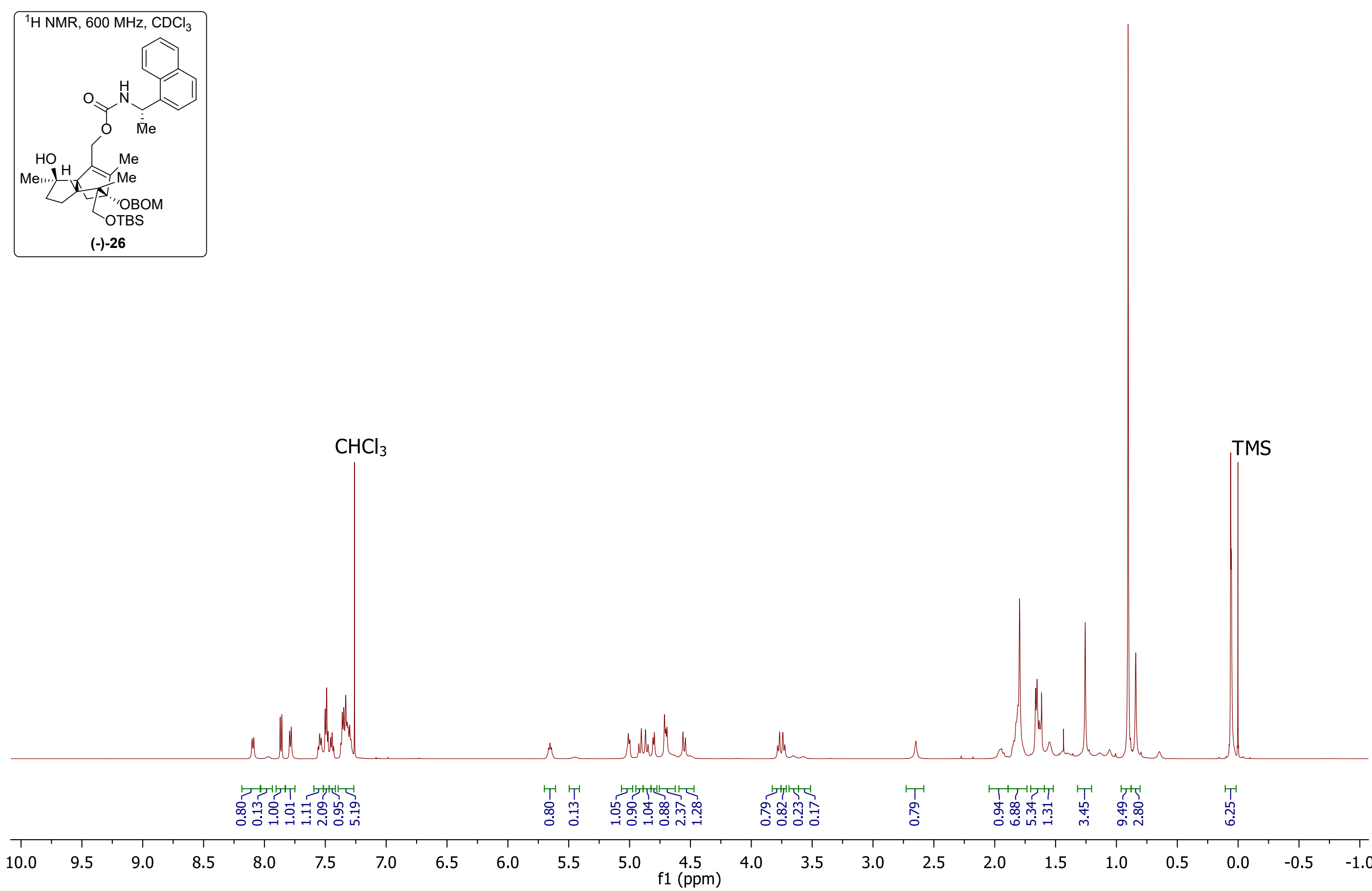


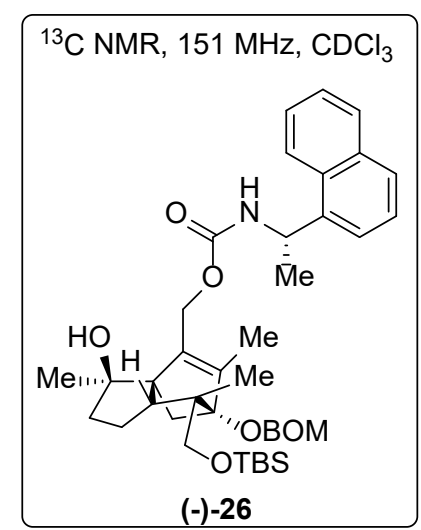

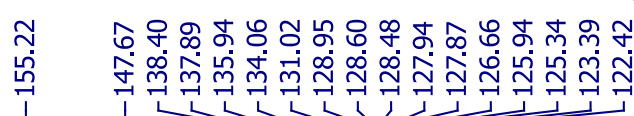

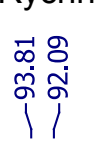

no

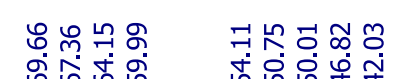

용요용

Page S99
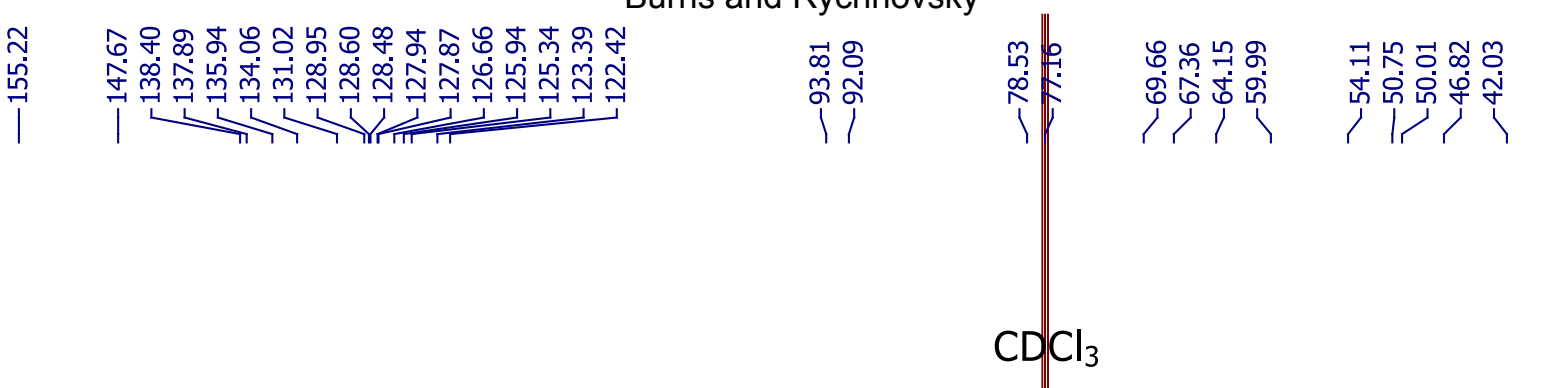

궁

$(-)-26$ 

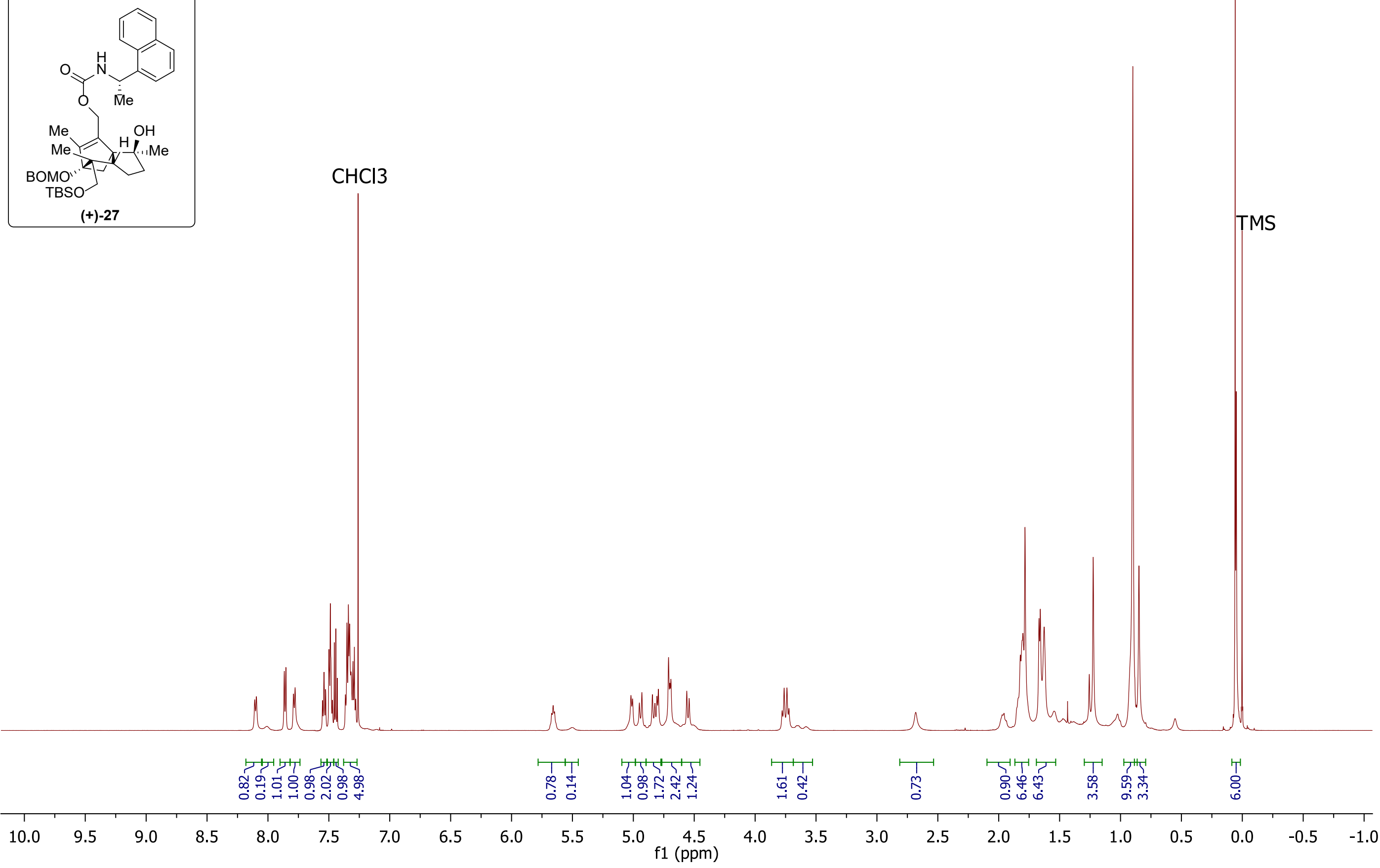


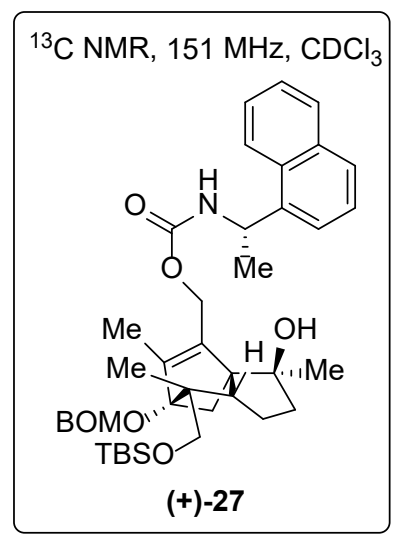

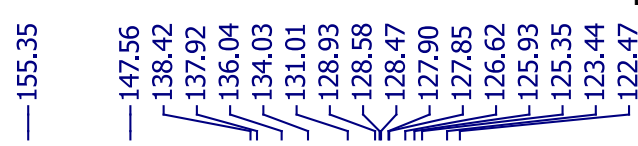

임

둥유ำ 슬

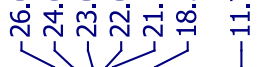




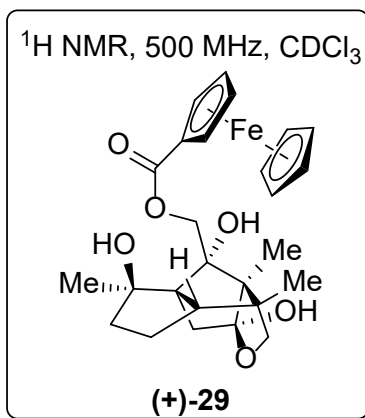

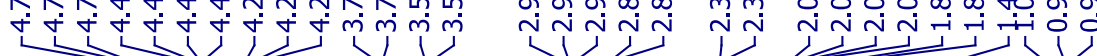

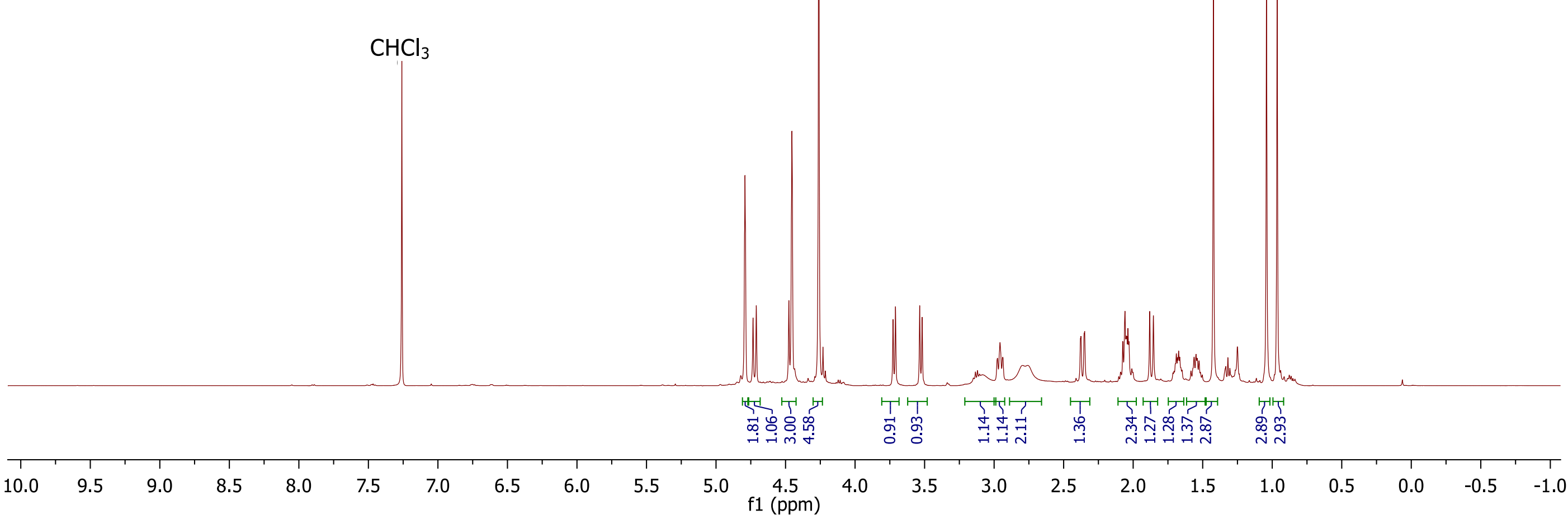



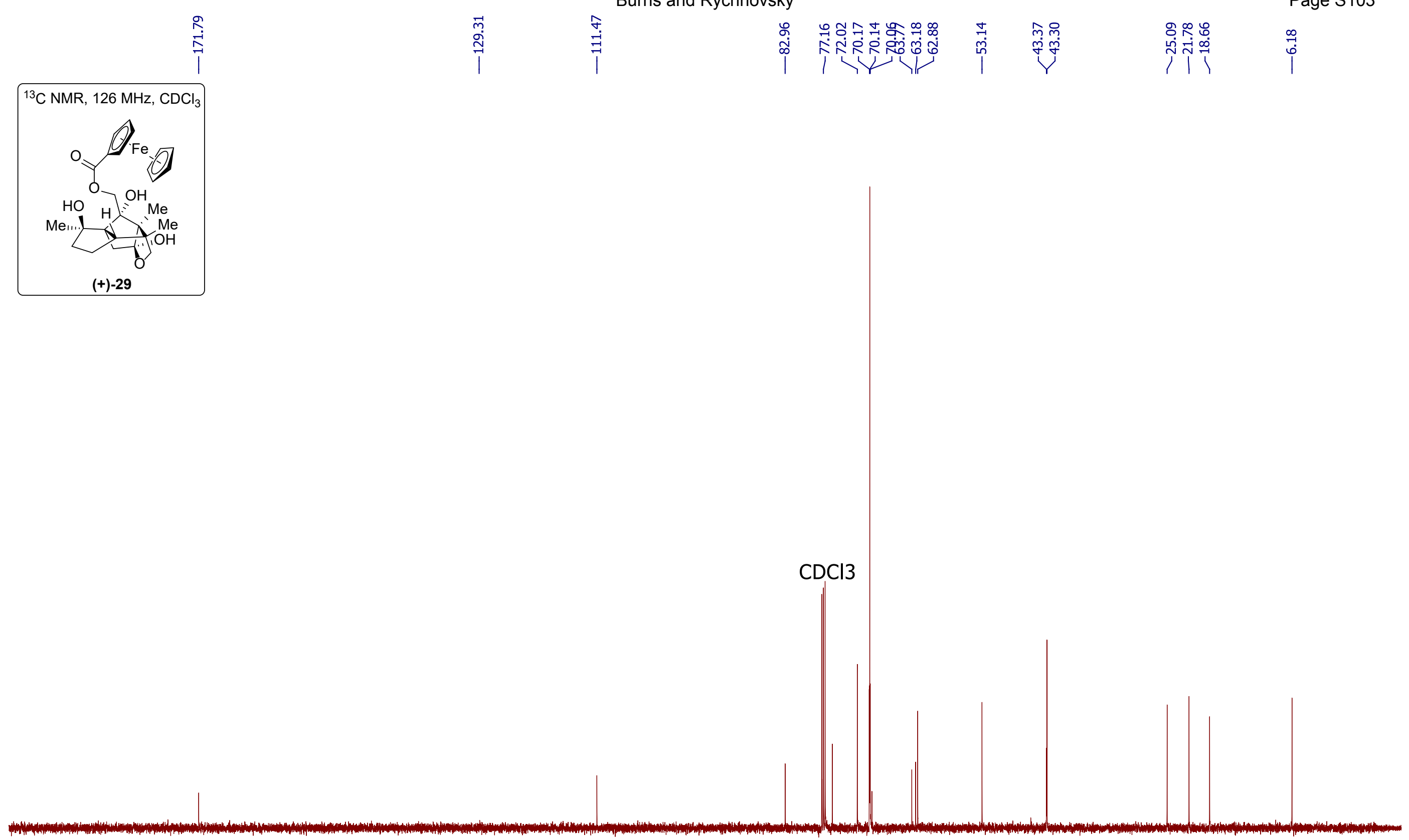

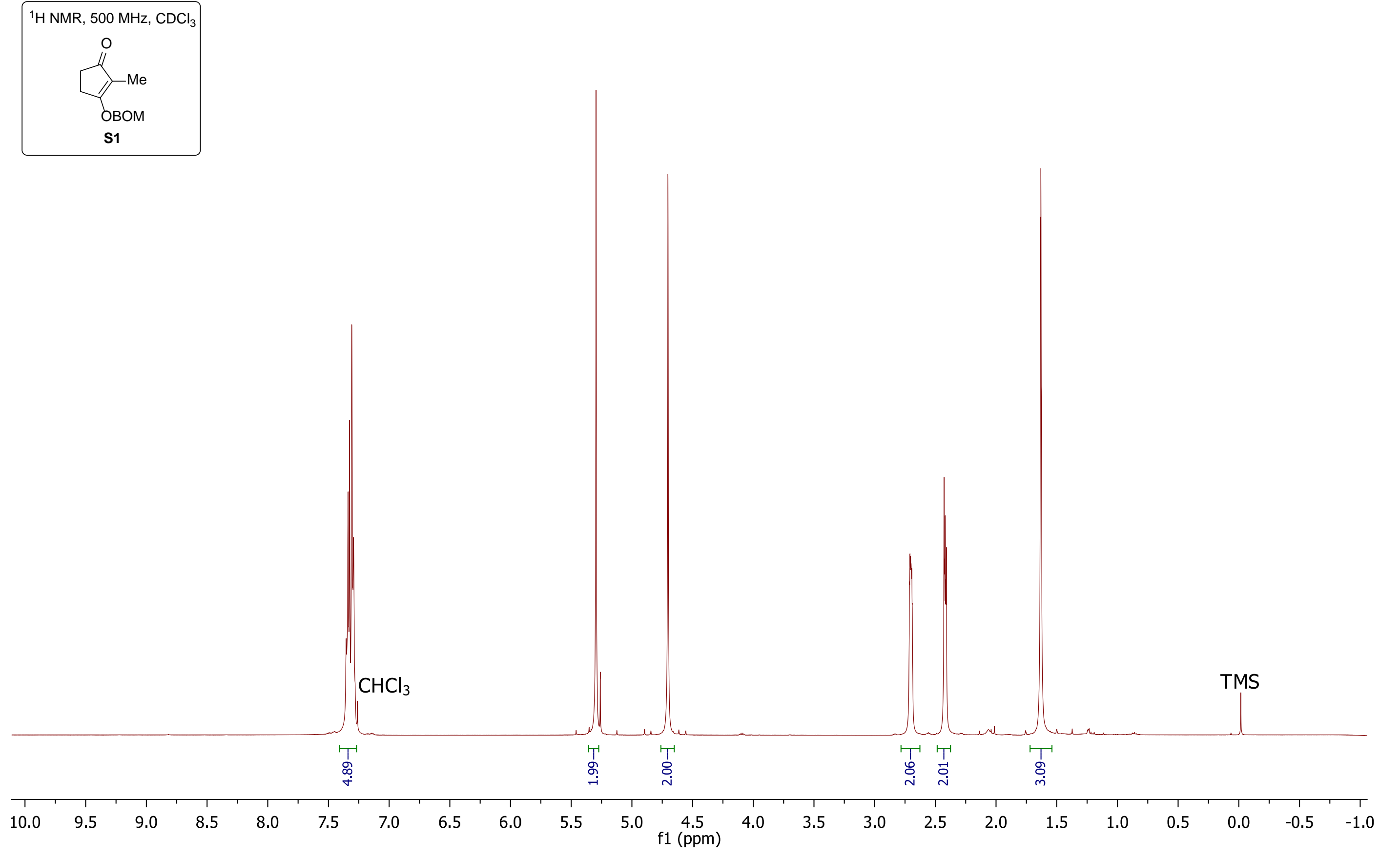
Burns and Rychnovsky

i⿱
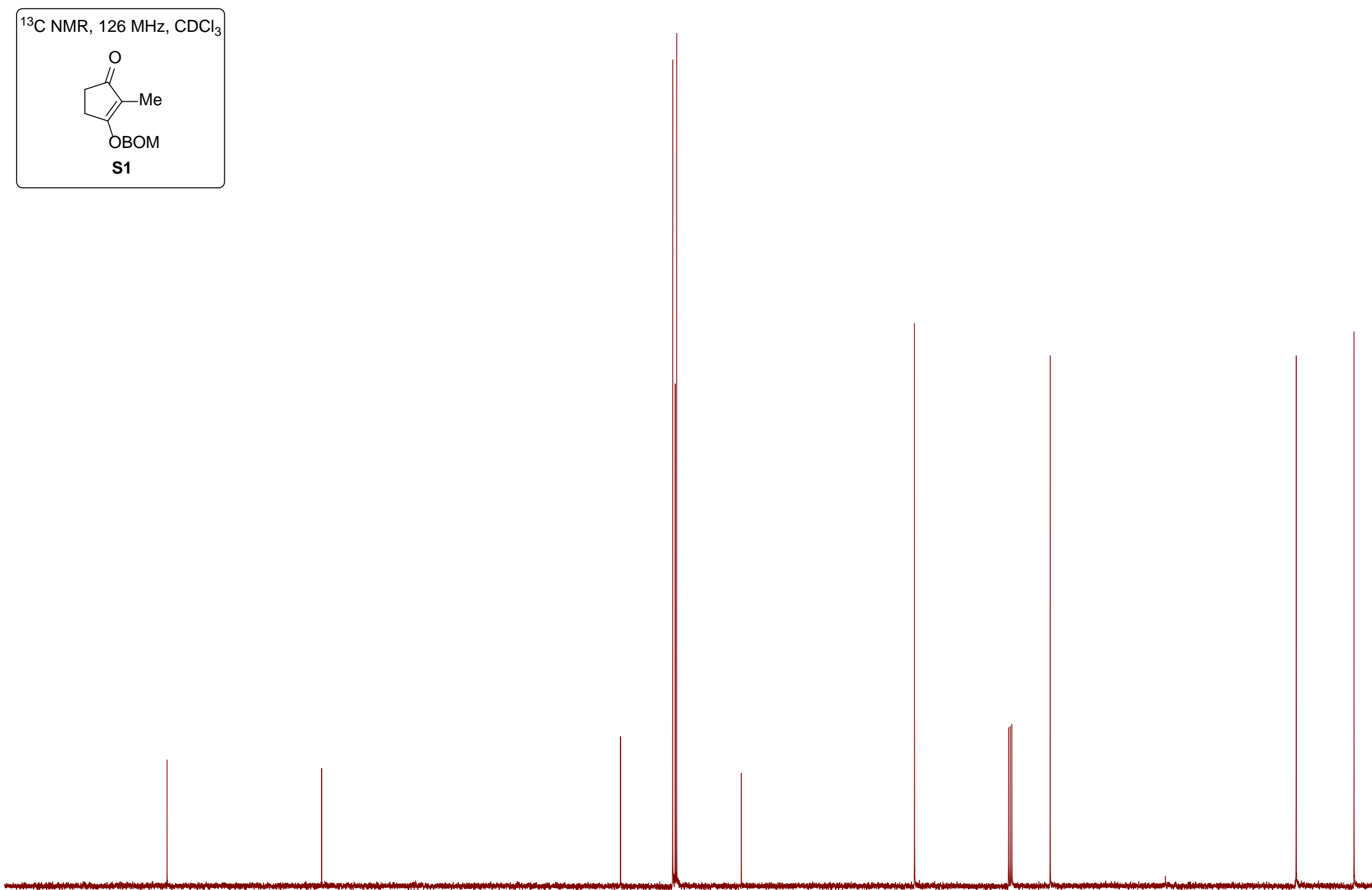
${ }^{1} \mathrm{H} \mathrm{NMR}, 500 \mathrm{MHz}, \mathrm{CDCl}_{3}$

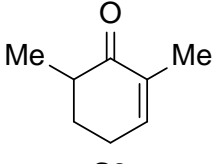

S3

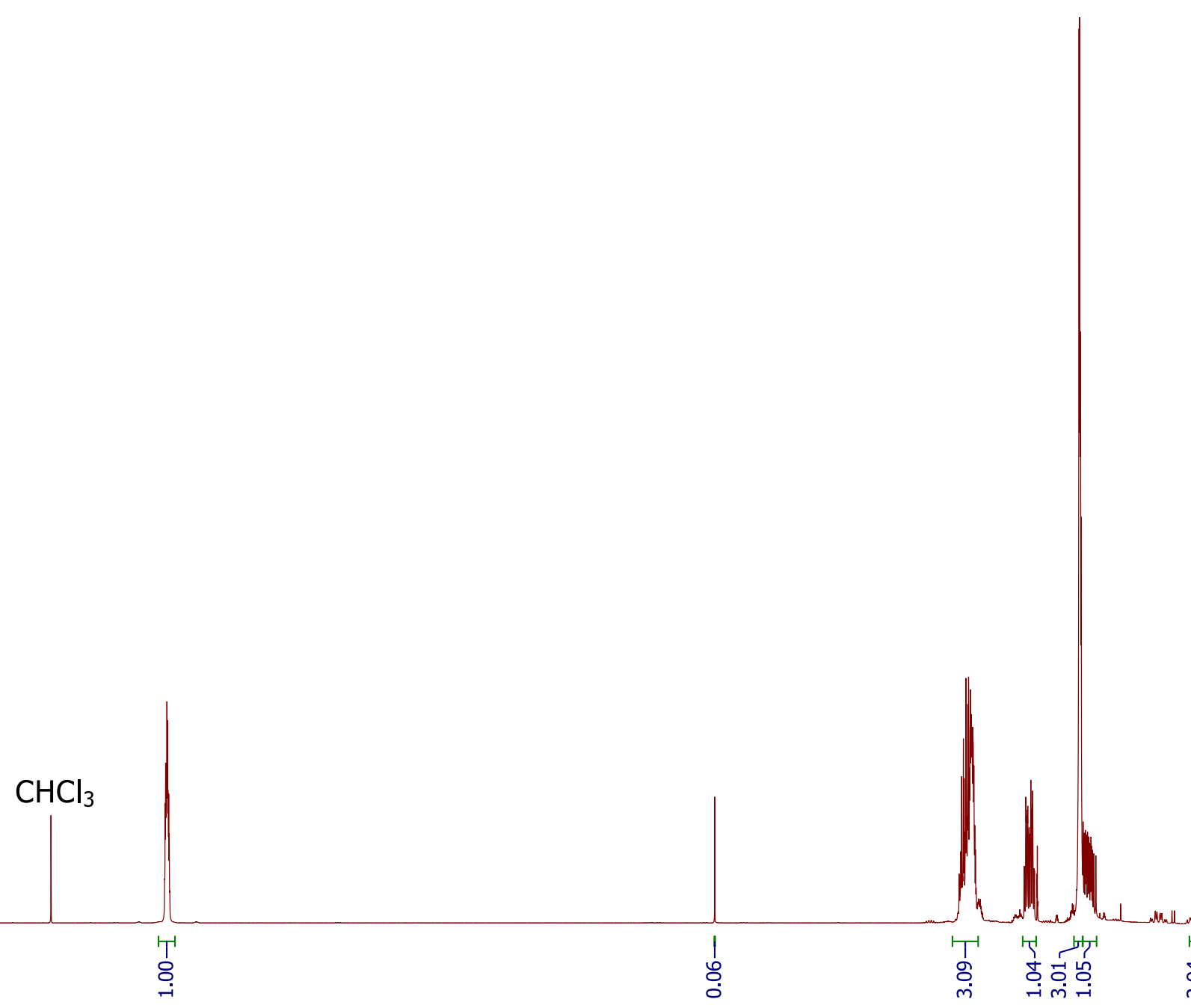



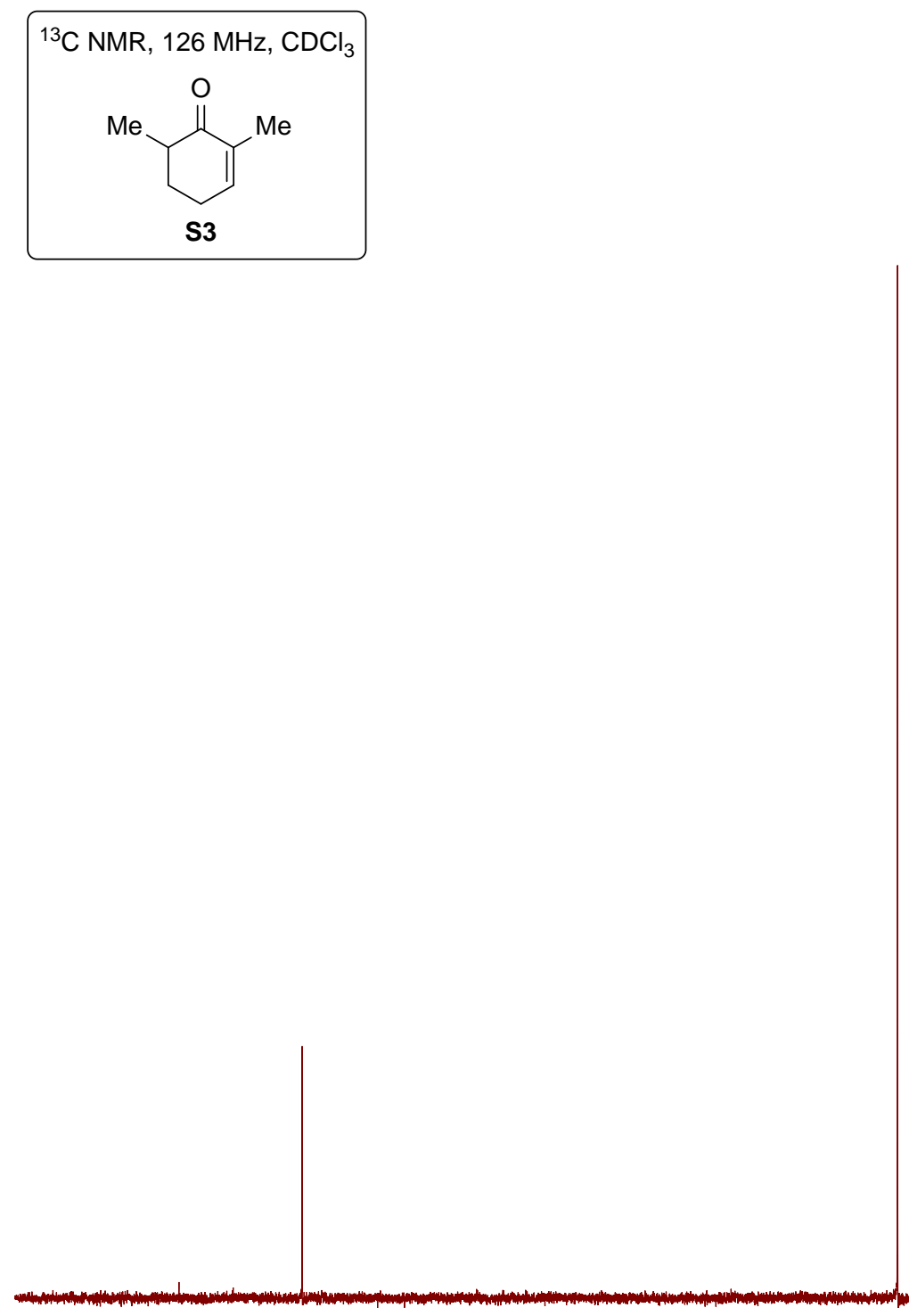

$\mathrm{CDCl}_{3}$ 
${ }^{1} \mathrm{H} \mathrm{NMR}, 500 \mathrm{MHz}, \mathrm{CDCl}_{3}$

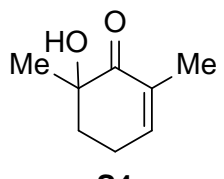

s4
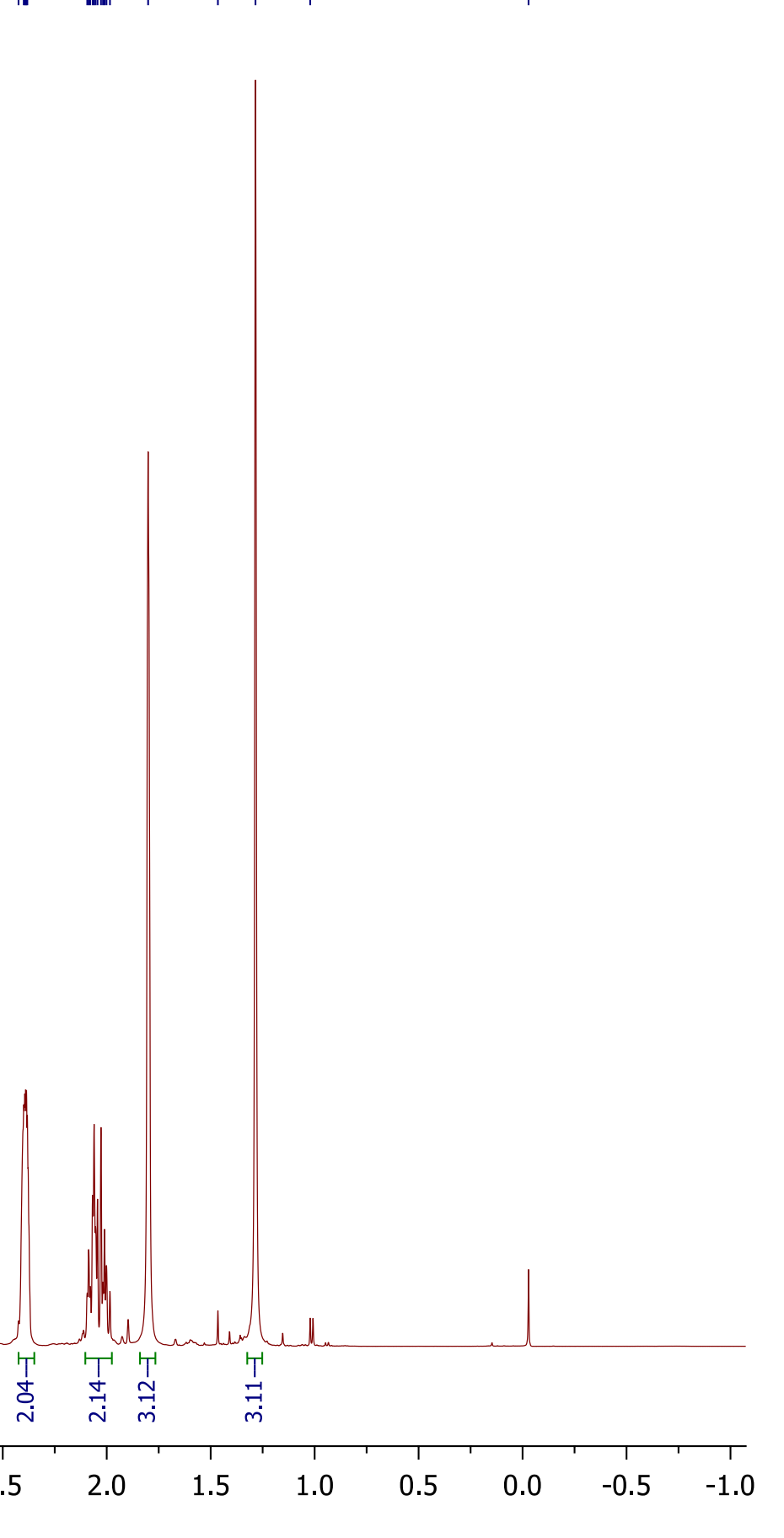

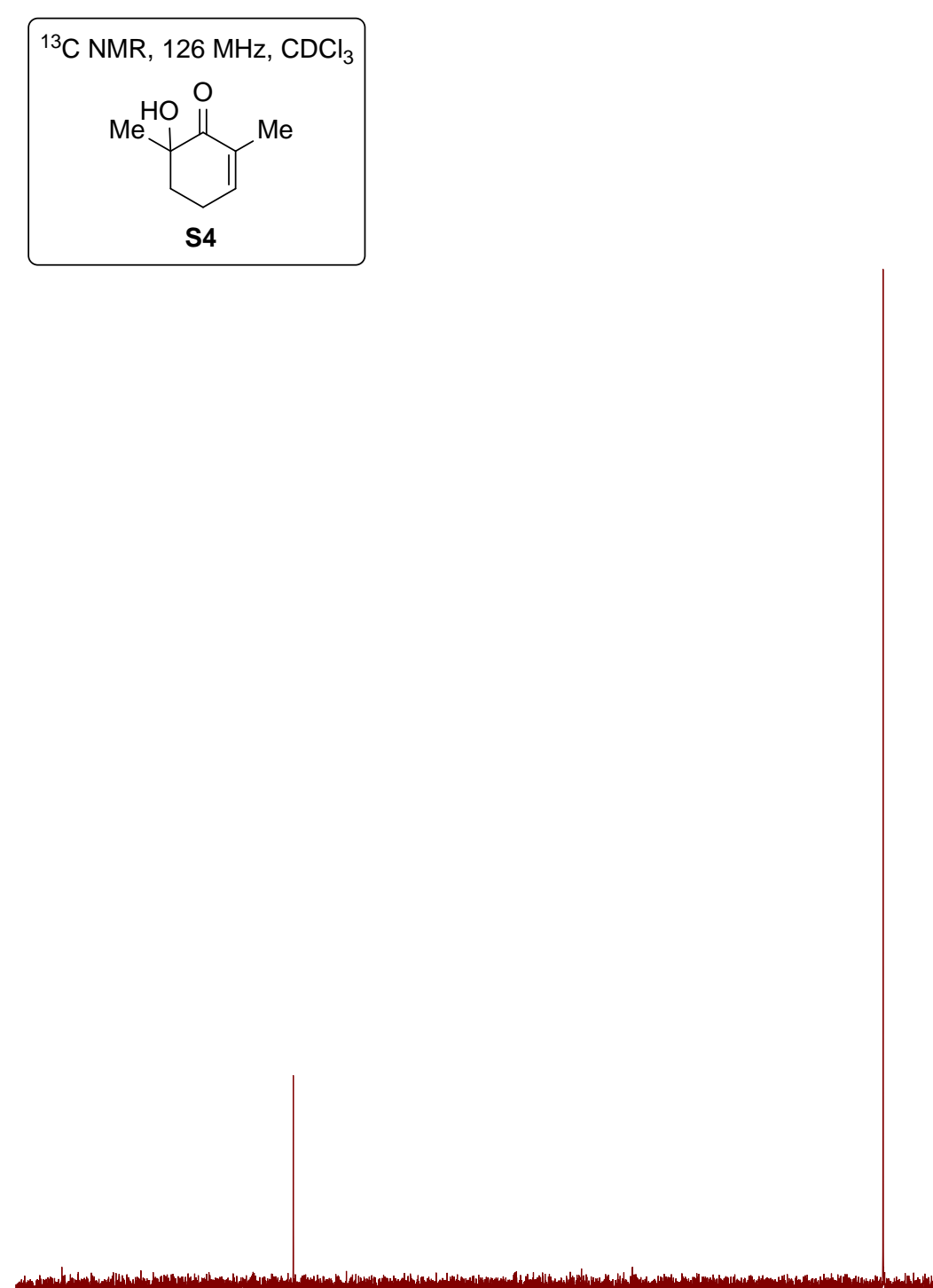

$\mathrm{CDCl}_{3}$ 

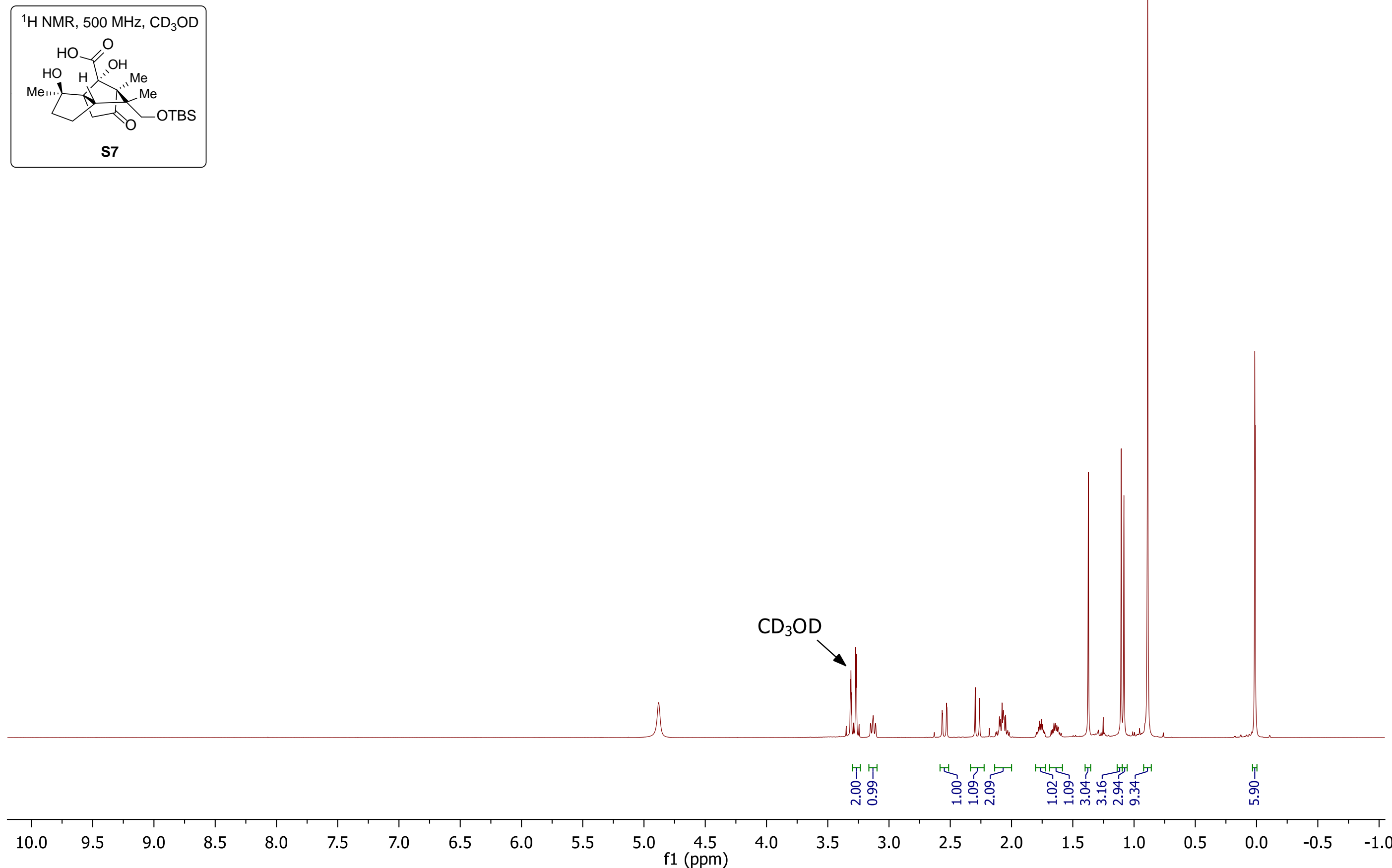


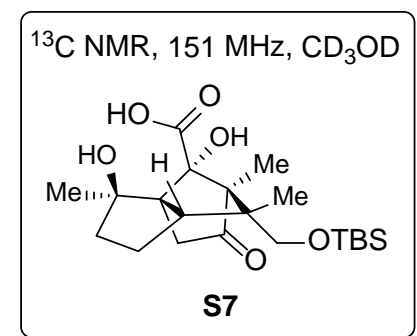




${ }^{1} \mathrm{H}$ NMR, $500 \mathrm{MHz}, \mathrm{CDCl}_{3}$

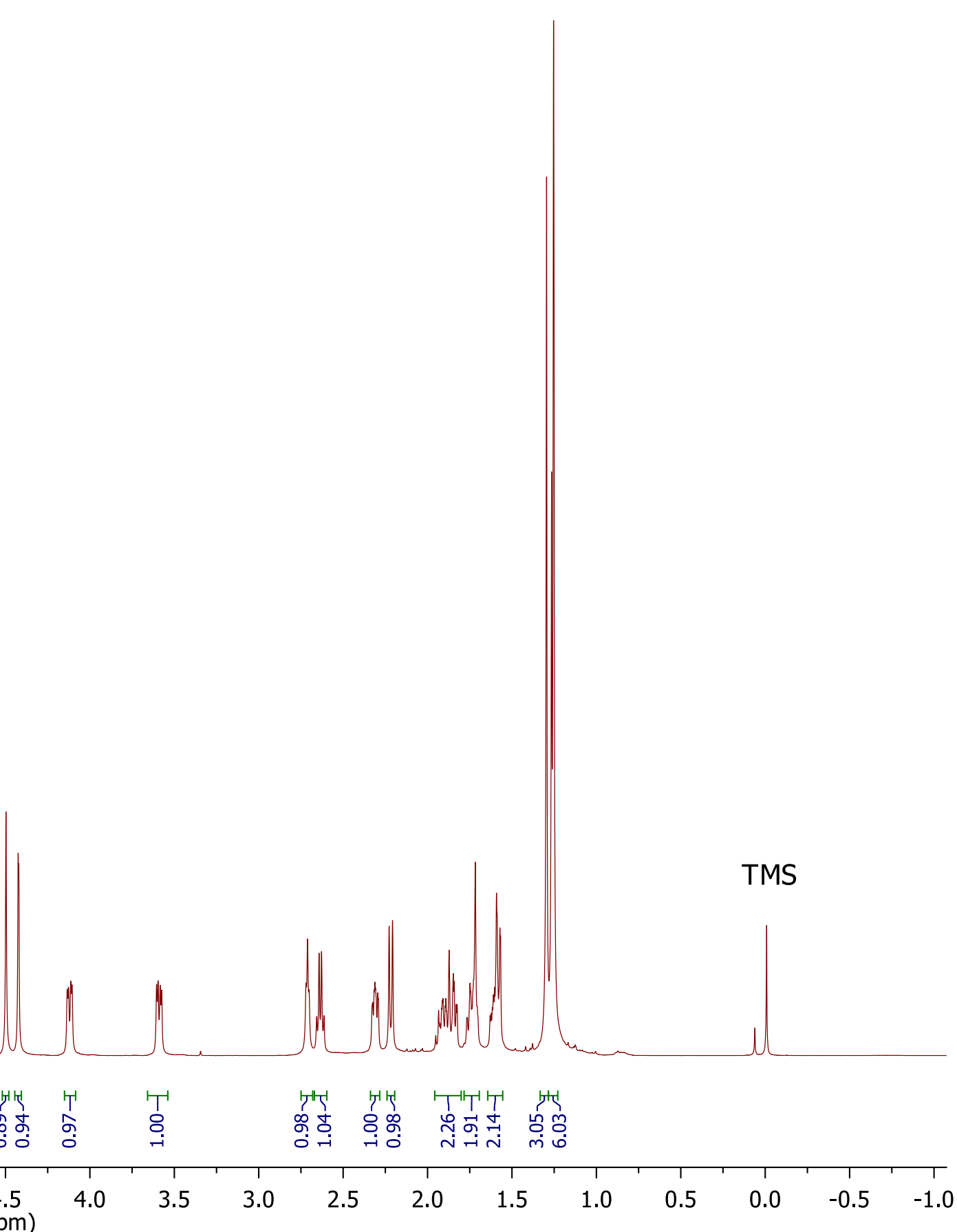




${ }^{13} \mathrm{C} \mathrm{NMR}, 126 \mathrm{MHz}, \mathrm{CDCl}_{3}$

\section{$\mathrm{CDCl}_{3}$}

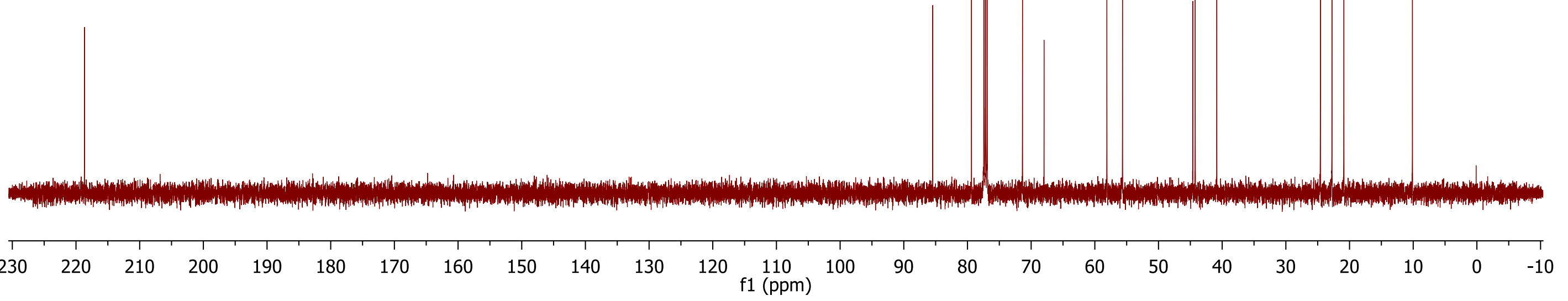



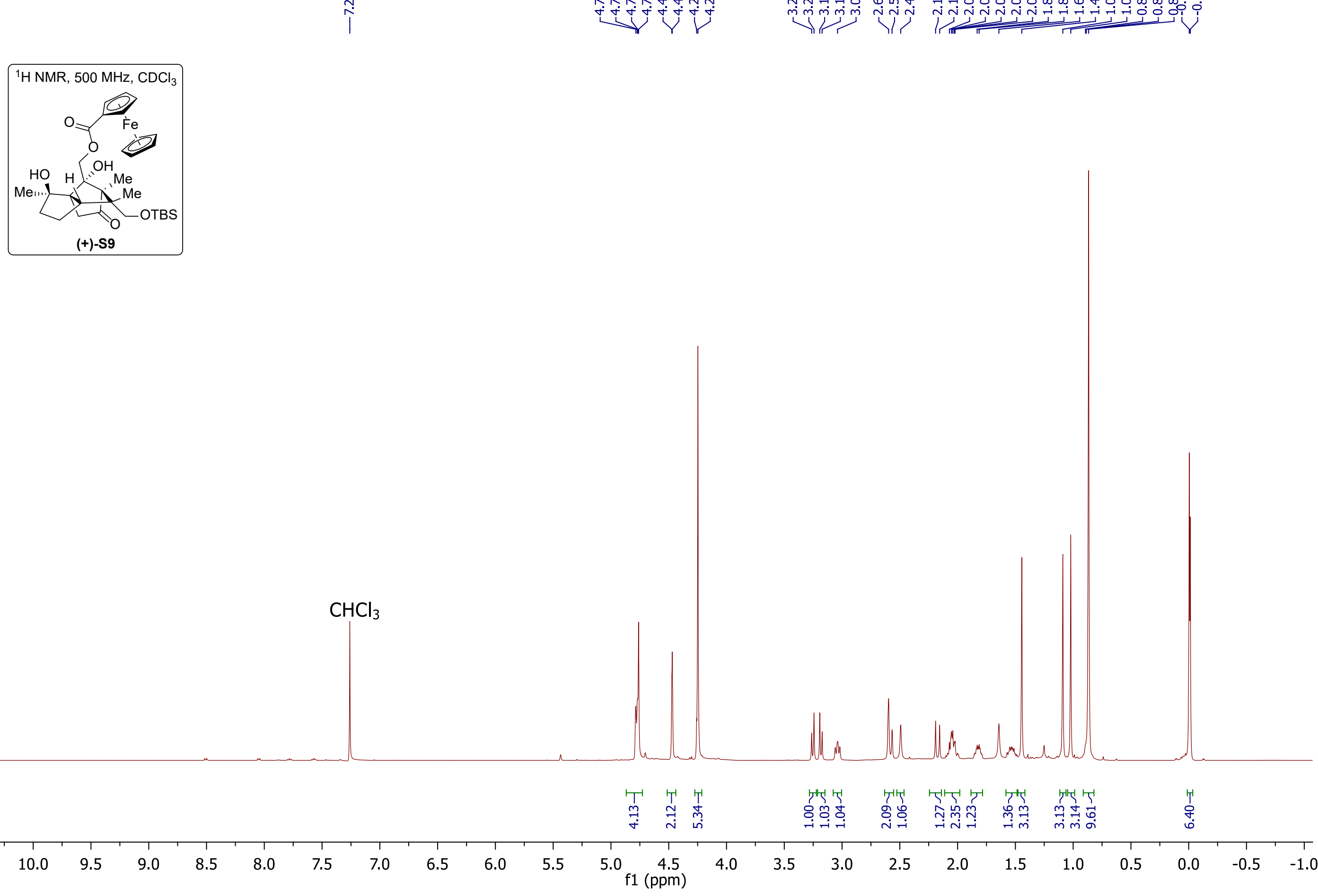

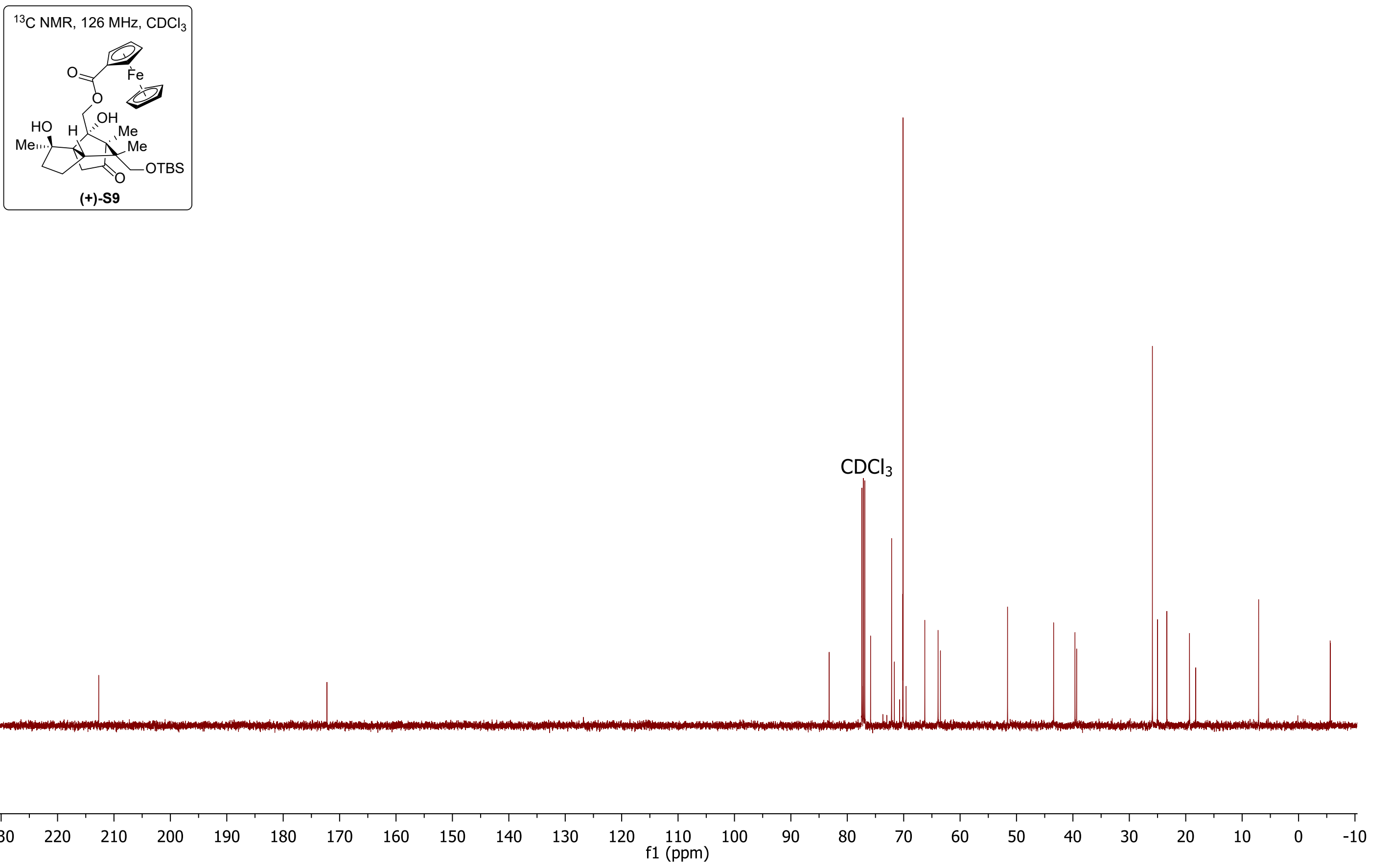
Chiralcel AD-H $w /$ ad guard, 2\%IPA/Hex, 20uL injection, 1. OmL/min, $2.15 \mathrm{~nm}$

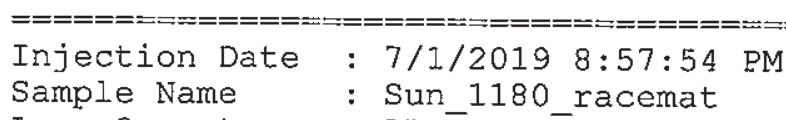

Acq. Operator

Acq. Method

Last changed

: ALEX $\bar{X}$

: C: $\backslash$ HPCHEM $\backslash 1 \backslash M E T H O D S \backslash A L E X B . M$

: 7/1/2019 8:56:11 PM by ALEXB (modified after loading)

Analysis Method : C: \HPCHEM $1 \backslash$ METHODS $\backslash A I E X B . M$

Last changed : 6/20/2019 1:38:43 AM by ALEXB

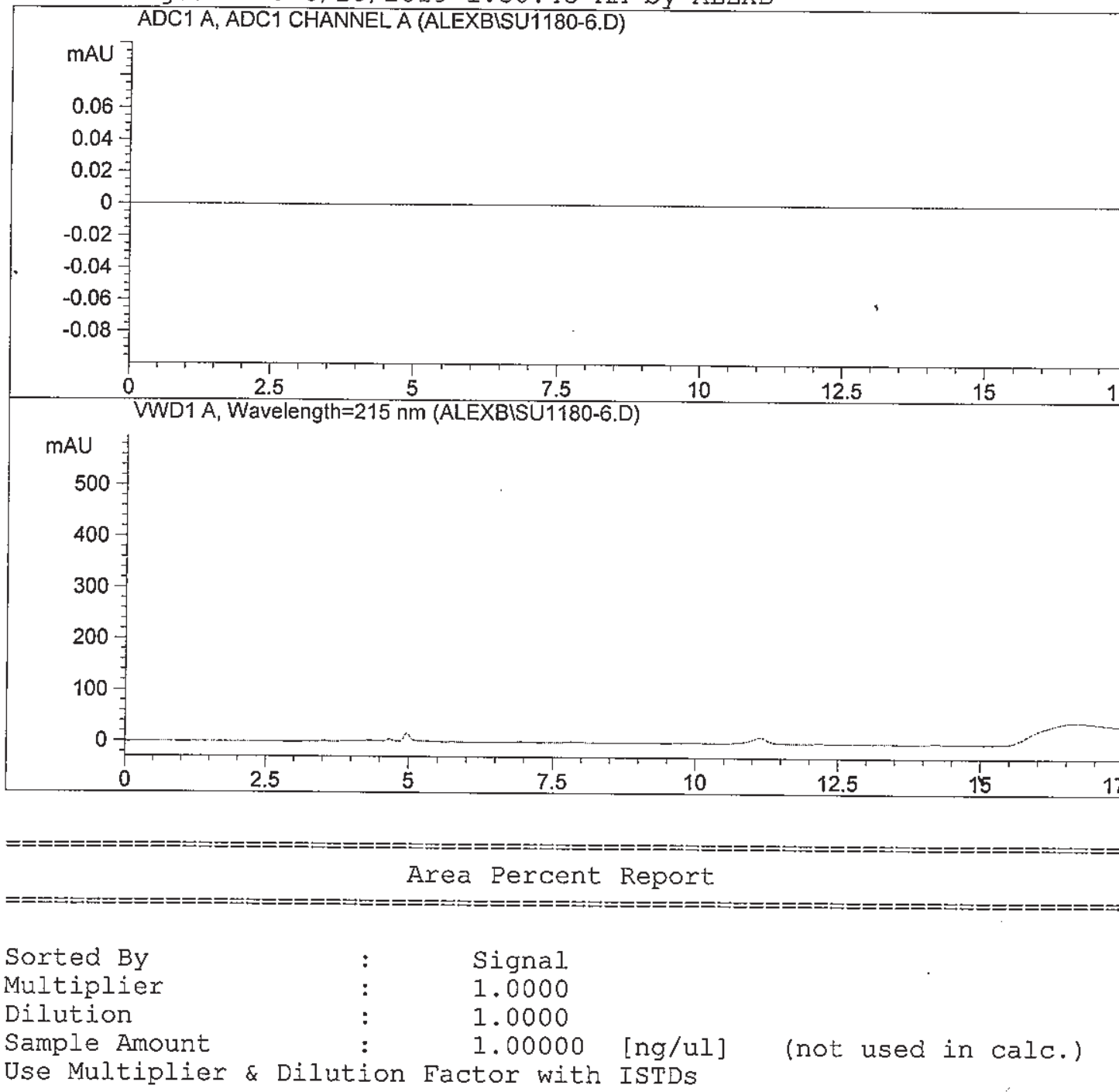

Location : Vial 26

Inj Volume : $40 \mathrm{\mu l}$

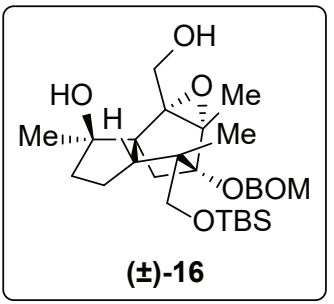

$( \pm)-16$

$\begin{array}{lll}7.5 & 20 & 22.5 \\ \end{array}$

Signal 1: ADC1 A, ADC1 CHANNEI A

Signal 2: VWD1 A, Wavelength=215 nm

\begin{tabular}{|c|c|c|c|c|c|c|}
\hline \multirow{2}{*}{$\begin{array}{c}\text { Peak } \\
\quad \#\end{array}$} & \multirow{2}{*}{$\begin{array}{c}\text { RetTime } \\
\text { [min] }\end{array}$} & \multirow{2}{*}{ Type } & \multirow{2}{*}{$\begin{array}{l}\text { Width } \\
{[\mathrm{min}]}\end{array}$} & Area & Height & \multirow{2}{*}{$\begin{array}{c}\text { Area } \\
\quad \text { 옹 }\end{array}$} \\
\hline & & & & $m A U \quad * s$ & {$[\mathrm{mAU}]$} & \\
\hline & & & & & & \\
\hline 2 & & $\begin{array}{l}\text { VV } \\
\text { VP }\end{array}$ & 0. & $5285 e 4$ & & 48 \\
\hline
\end{tabular}

Totals :

$$
4.20702 \mathrm{e} 4 \quad 1031.79727
$$

Results obtained with enhanced integrator! 
Chiralcel $\mathrm{AD}-\mathrm{H} w /$ ad. guard, $2 \% \mathrm{IPA} / \mathrm{Hex}, 20 \mathrm{uL}$ injection, 1. $0 \mathrm{~mL} / \mathrm{min}, 215 \mathrm{~nm}$

$=====-:=====$
Injection Date

Sample Name

Acq. Operator

Acq. Method

Last changed

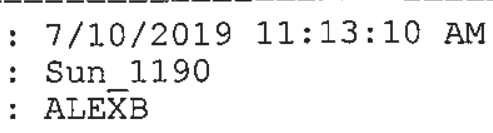

: C: $\backslash$ HPCHEM $\backslash 1 \backslash$ METHODS $\backslash A L E X B, M$

: 7/10/2019 11:06:40 AM by ALEXB (modified after loading)

Analysis Method : C: $\backslash$ HPCHEM $\backslash 1 \backslash$ METHODS $\backslash A L E X B . M$
Location : Vial 21

Inj Volume : $20 \mu l$

Last changed : 6/20/2019 $1: 38: 43$ AM by ALEXB

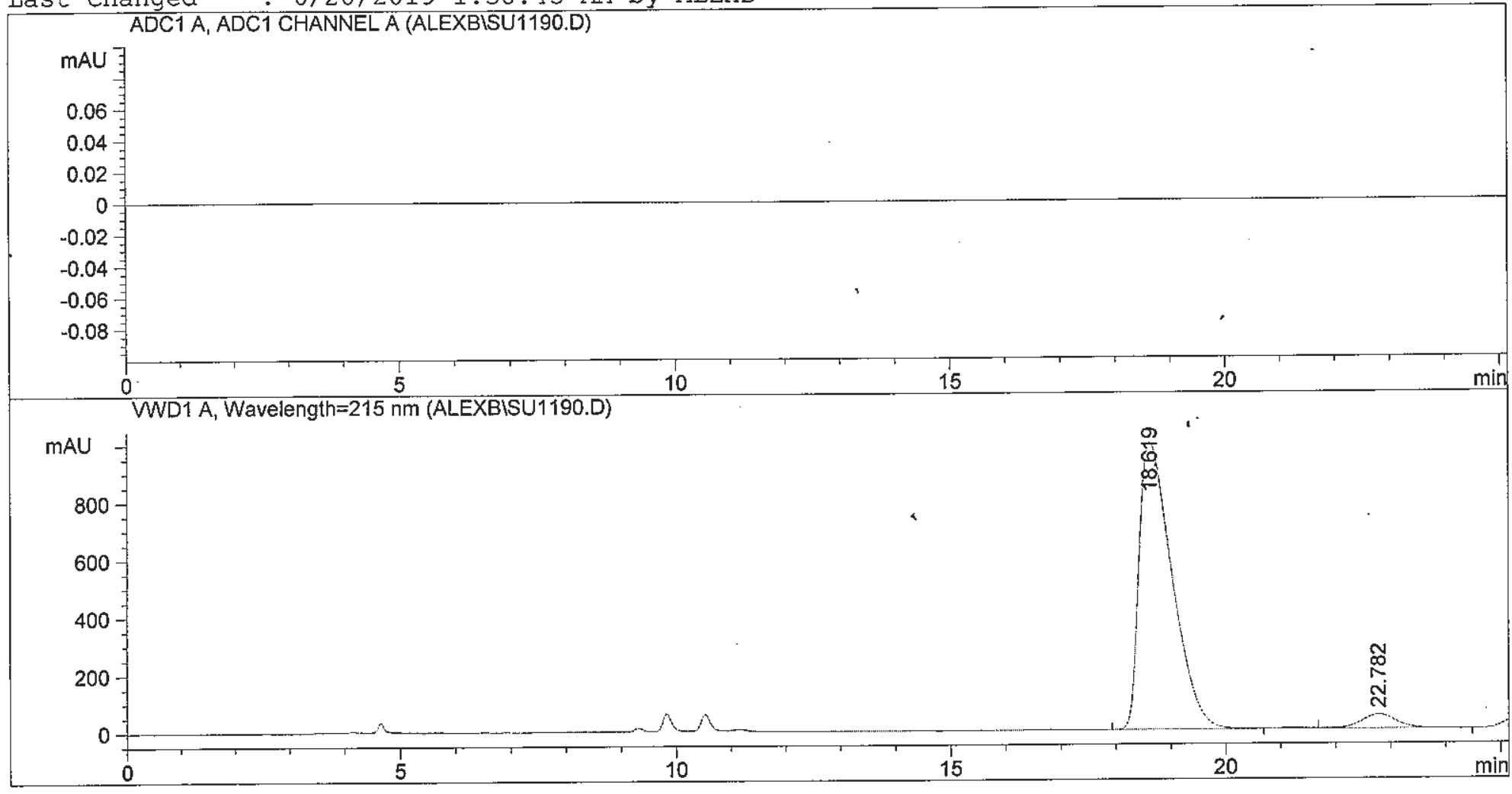

\section{Area Percent Report}

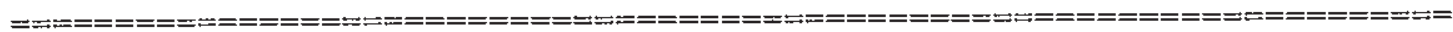

Sorted By : : Signal

Multiplier : : 1.0000

Dilution : $\quad 1.0000$

Sample Amount : $1.00000 \quad[\mathrm{ng} / \mathrm{ul}]$ (not used in calc.)

Use Multiplier \& Dilution Factor with ISTDs

Signal. 1: ADC1 A, ADC1, CHANNEL A

Signal 2: VWD1 A, Wavelength=215 nm

\begin{tabular}{|c|c|c|c|c|c|c|c|}
\hline \multirow{2}{*}{$\begin{array}{c}\text { Peak } \\
\#\end{array}$} & \multirow{2}{*}{$\begin{array}{l}\text { RetTime } \\
\text { [min] }\end{array}$} & \multirow{2}{*}{ Type } & \multirow{2}{*}{$\begin{array}{c}\text { Width } \\
\text { [min] }\end{array}$} & \multicolumn{2}{|c|}{ Area } & Height & \multirow{2}{*}{$\begin{array}{c}\text { Area } \\
\text { \% }\end{array}$} \\
\hline & & & & $M A U$ & *s & {$[\mathrm{mAO}]$} & \\
\hline & & & & & & & \\
\hline 1 & 18.619 & VB & 0.6 & 4. & 0 e 4 & 87323 & 49 \\
\hline 2 & 22.782 & VP & 0.6961 & 2263 & 7002 & 49.40286 & 51 \\
\hline
\end{tabular}

Totals :

$$
4.39033 e 4 \quad 1049.27609
$$

Results obtained with enhanced integrator! 
Chiralcel AD-H w/ ad guard, 2IPA/Hex, 20uL injection, 1. $0 \mathrm{~mL} / \mathrm{min}, 215 \mathrm{~nm}$

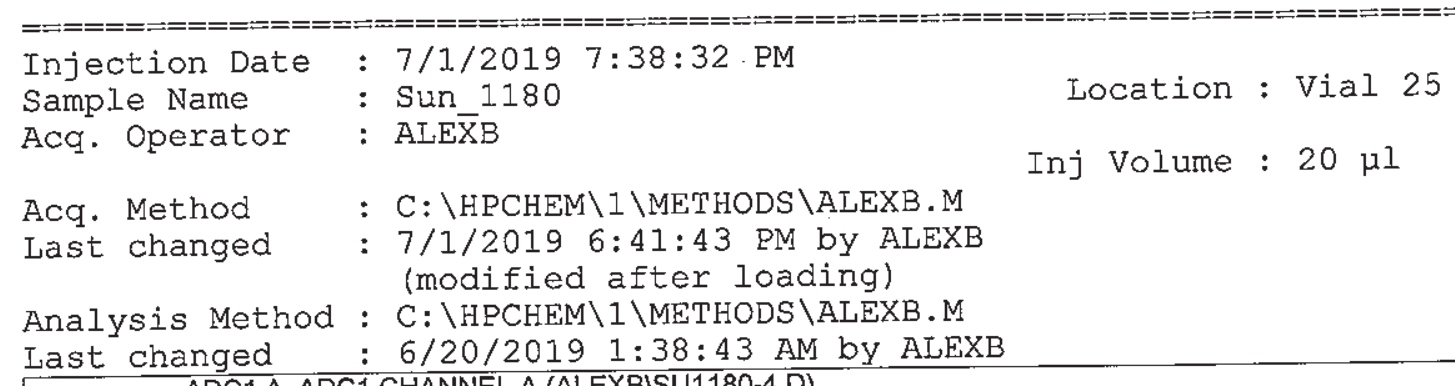

Analysis Method : C: \HPCHEM \1\METHODS \ALEXB.M

Iast changed : 6/20/2019 1:38:43 AM by ALEXB

Location : Vial 25

Inj Volume : $20 \mu l$

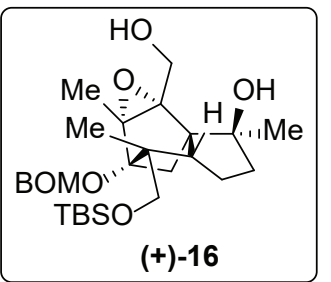

$(+)-16$

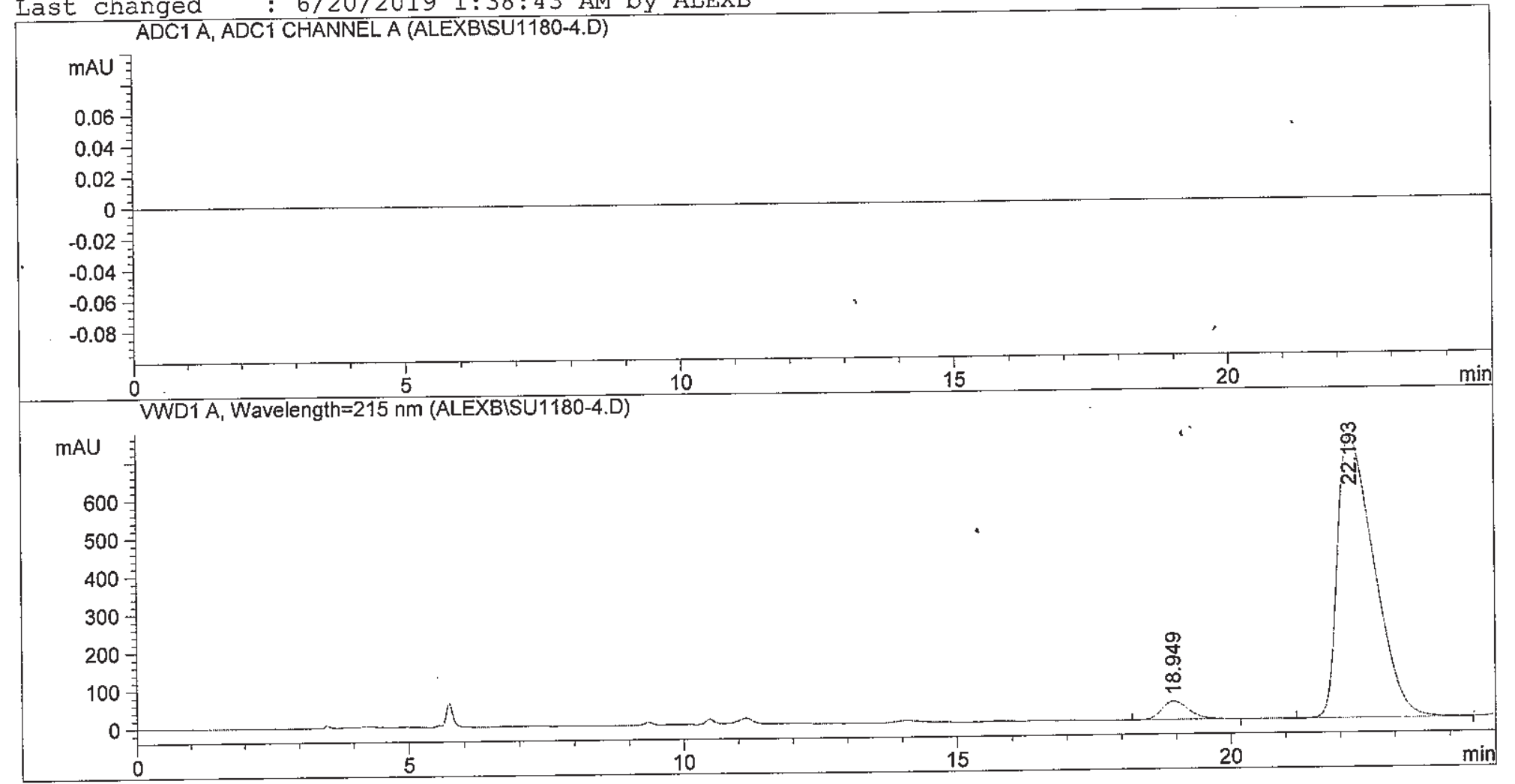

Area Percent Report

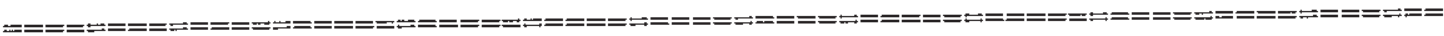

Sorted By : : Signal

Multiplier : $\quad 1.0000$

Dilution : $\quad 1.0000$

Sample Amount : 1.00000 [ng/ul] (not used in calc.)

Use Multiplier \& Dilution Eactor with ISTDs

Signal 1: ADCl A, ADCi CHANNEL A

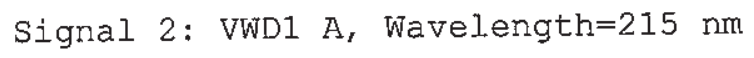

\begin{tabular}{|c|c|c|c|c|c|c|}
\hline eak & $\operatorname{Ret}$ & Type & Width & Area & Height & Area \\
\hline \# & [min] & & [min] & mAU & {$[\mathrm{mAU}$} & \\
\hline 1 & & VV & & $.45933 e 4$ & $\begin{array}{r}48.68089 \\
741.82263\end{array}$ & \\
\hline
\end{tabular}

Totals :

$$
3.63017 e 4 \quad 790.50352
$$

Results obtained with enhanced integrator! 